CONTRIBUTION A L'ETUDE DE LA VEGETATION DANS LE BAER-BASSIT ET LE DJEBEL ALAOUITE DE SYRIE

(MET EEN SAMENVATTING IN HET NEDERLANDS; WITH A SUMMARY IN ENGLISH; CON UN RIASSUNTO IN ITALIANO) 
Dit proefschrift met stellingen van

\section{IBRAHIM NAHAL}

Ingénieur Agricole (Ecole Nationale d'Agriculture de Grignon), Ingénieur Civil des Eaux et Forêts (Ecole Nationale des Eaux et Forêts, Nancy), geboren te Lattaquié, Syrië, 2 april 1933 , is goedgekeurd door de promotor, Dr. H. J. Venema, hoogleraar in de plantensystematiek,dendrologie en plantengeografie.

De Rector Magnifious van de Landbouwhogeschool, W. F. EiJstoogeL

Wageningen, 7 mei 1962. 


\title{
CONTRIBUTION A L'ETUDE DE LA VEGETATION DANS LE BAER-BASSIT ET LE DJEBEL ALAOUITE DE SYRIE
}

\author{
PROEFSCHRIFT \\ TER VERKRIJGING VAN DE GRAAD \\ VAN DOCTOR IN DE LANDBOUWKUNDE \\ OP GEZAG VAN DE RECTOR MAGNIFICUS, IR. W. F. EIJSVOOGEL, \\ HOOGLERAar IN DE hYDRAULICA, DE BEVLOEIING, \\ DE WEG- EN WATERBOUWKUNDE EN DE \\ BOSBOUWARCHITECTUUR, \\ TE VERDEDIGEN TEGEN DE BEDENKINGEN \\ VAN EEN COMMISSIE UIT DE SENAAT \\ VAN DE LANDBOUWHOGESCHOOL TE WAGENINGEN \\ OP VRIJDAG, 6 Juli 1962 TE 16 UUR \\ DOOR \\ I. NAHAI,
}


Blbllotheok dor

Landbouw Hogeschool

WAGENINGEN 
CONTRIBUTION A L'ETUDE DE LA VEGETATION

DANS LE BAER-BASSIT ET LE DJEBEL ALAOUITE DE SYRIE

\section{THÈSE}

présentée à l'Université d'Agriculture à Wageningen pour obtenir le grade de Docteur en agronomie

par

I. NAHAL

Ing. Agr. de Grignon

Ing. Civil des Eaux et Forêts, Nancy 
A mes parents

A tous mes professeurs 


\section{I}

Sous le climat méditerranéen, une forte luminosité est nécessaire anx espèces d'origine septentrionale, pendant leur jeune âge (comme le sapin et le hêtre) pour pouvoir resister à l'aridité estivale.

Gracobre A. 1955 : Les effets écologiques de l'aridité du climat sur la végétation méditerranéenne : héliophilie et configuration morphologique des forêts. In : Ecologie Végétale; Actes du Colloque de Montpellier, UNESCO, pp. 64-67.

\section{II}

Dans les zones arides, parmi les caractères physico-chimiques des sols jouant un rôle dans la biologie des plantes, la texture est des plus importants.

LEMÉe G. 1955 : Exposé général sur les rapports entre sols et végétation. In : Ecologie Végétale; Actes du Colloque de Montpellier, UNESCO ; pp. 73.77.

\section{III}

La notion de Climax climatique de la végétation est très discutable dans les régions arides et doit être remplacée par la notion de climax édaphique.

VERNET A. 1958 : Climat et végétation. In : Climatologie; UNESCO, pp. 84-110.

WitTaker R. H. 1953 : A consideration of climax theory : the climax as a population and pattern. Ecol. monogr., 23, I, 41-78.

\section{IV}

Dans les régions arides, l'étude des climats régionaux est insuffisante pour l'établissement des projets de developpements agronomiques et forestiers et il est nécessaire de procéder à une étude détaillée des microclimats.

\section{V}

La composition ohimique de l'essence de térébenthine dans le genre Pinus a un caractère specifique.

Mrrov. N. T. 1961 : Composition of Gum Turpenines of Pinus. U. S. Dept. of Agric. ; Tech. Bull. No 1239, pp. 158.

\section{VI}

L'assèchement intensif des zones steppiques de Syrie est avant tout une conséquence de l'action humaine. 


\section{VII}

Le principe classique du rapport soutenu de la sylviculture septentrionale est souvent inapplicable dans les forêts méditerranéennes et doit être négligé dans la majorité des cas.

Boudx P. 1950 : Economie forestière Nord-Africaine; T. 2, fasc. 2, Paris.

\section{VIII}

La "terra rossa " est un sol fossile se comportant comme une roche mère qui évolue sous l'influence du climat et de la végétation actuels.

Duchaurour 1960 : Précis de Pedologie. Masson et Cie, Paris.

\section{IX}

La rubéfaction dans les sols est un phénomène qui continue à se produire sous le climat méditerranéen actuel.

$\mathbf{X}$

Les reboisements en Syrie doivent être établis sur des bases écologiques et économiques.

Diss., I. Nahal

Wageningen, Mai 1962 


\section{CONTRIBUTION A L'ETUDE DE LA VEGETATION DANS LE BAER-BASSIT ET LE DJEBEL ALAOUITE DE SYRIE}

- La végétation donne a qui sait la lire les indications les plus préciges sur les possibilités de l'expansion hamaine s.

Ch. Fuafault

\section{INTRODUCTION}

En 1956, nous avons eu la chance d'être affecté au Service Forestier du Mohafazat (Département) de Lattaquié qui est le plus important de Syrie du point de vue forestier. Grâce à sa position géographique côtière et son climat privilégié, on y tronve les plus belles forêts de Syrie: forêts de Pinus brutia, Quereus Cerris, Quercus pseudocerris, Quercus calliprinos, Cedrus libani, Abies cilicica.

Les forêts de Pinus brutia y recourrent de grandes superficies et constituent ainsi, des réserves de bois assez importantes pour la Syrie. Mais, ces forêts souffrent de l'absence d'une sylviculture appropriée et d'un aménagement convenable.

En outre, toutes les forêts du Mohafazat de Lattaquié, ont subi l'influence des anciennes civilisations du Proche-Orient depuis des millénaires et sont, de ce fait, en désordre et en grande partie assez dégradées.

L'aménagement de ces forêts et leur mise en valeur, ainsi que l'ellaboration d'une sylviculture appropriée nécessite, en premier lieu, l'étude de la végétation, de son écologie et de son évolution sous l'influence des facteurs du milieu et en particulier sous l'influence de l'homme.

* Ingénieur agricole, Ingénieur Civil des Eaux et Forêts, Ministère de l'Agriculture, Damas, Syrie.

In redazione il 23 Dicembre 1961. 
Jusqu'à cette époque, aucune étude précise et valable sur les bioclimats, les sols et la végétation, n'a été entreprise dans cette région. Nous nous sommes penché done, non sans passion, ̀̀ l'étude des bioclimats, des sols et de la végétation des deux principales régions forestières : le Baer-Bassit et le Djebel Alaouite (Dj. Ansarieh).

Les résultats que nous présentons ici sont le fruit de nos recherches entreprises dans ces régions de 1956 à 1960 .

Dans la réalisation de notre travail, nous avons beaucoup profité des Flores déjà écrites sur le Proche-Orient et en particulier sur la Syrie et le Liban.

Nous tenons ici à rendre hommage aux botanistes qui ont écrit des Flores sur la Syrie et le Proche-Orient et à tous ceux qui ont contribué à la connaissance de notre flore et rendu ainsi possible nos recherches sur la végétation du Baer-Bassit et du Djebel Alaonite.

Monsieur le Professeur Venema, Directeur du Laboratoire de Systématique végétale et de Phytogéographie de l'Université de Wageningen, a bien voulu nous communiquer ses observations et critiques sur les parties de ce mémoire concernant la flore et la végétation. Nous le prions de bien vouloir trouver ici le témoignage de notre reconnaissance.

A Monsieur le Professeur Emberger, Directeur de l'Institut de Botanique de Montpellier, nous sommes tout spécialement reconnaissant pour les multiples discussions sur les bioclimats que nous avons eues avec lui. Nous avons pu ainsi profiter de sa longue expérience dans l'étude des bioclimats méditerranéens.

Nous remercions vivement Monsieur le Professeur AUBERT, Directeur du Laboratoire des sols de l'I.D.E.R.T. à Bondy, pour les entretiens si fructueux que nous avons eus avec lui sur la pédogénèse dans la Région méditerranéenne, ainsi que pour ses critiques sur la partie de notre travail concernant les sols et leur classification.

C'est avec beaucoup de plaisir que nous remercions ici Monsieur Duchaufour, Professeur à l'Ecole Nationale des Eaux et Forêts de Nancy pour l'aide qu'il nous a apportée dans l'interprétation de quelques profils pédologiques.

Le Révérend Père Mouterde, Professeur à l'Université Saint- 
Joseph de Beyrouth, nous a communiqué très aimablement ses critiques sur les plantes endémiques du Baer-Bassit et du Djebel Alaouite; qu'il veuille trouver ici l'expression de notre reconnaissance.

Nous profitons de cette occasion pour adresser toute notre reconnaissance au Ministère de l'Agriculture de Syrie et tout particulièrement à la Direction des Forêts pour les facilités matérielles qu'ils nous ont accordées dans nos travaux de recherches. Nous remercions aussi tout le personnel du Service Forestier de Lattaquié pour le dévouement avec lequel il a collaboré avec nous.

Nous devons quelques analyses des sols formés sur roches vertes à notre ami, Monsieur Samouh BaYAN, de la Station de Recherches Agronomiques de Rayak au Liban. Nous le prions d'accepter nos remerciements.

Il nous est agréable d'exprimer ici notre reconnaissance et nos remerciements à tous ceux qui, à des titres divers, ont permis la réalisation de nos recherches et la rédaction finale de ce mémoire.

Enfin, nous adressons nos remerciements, tout particulièrement, à la Direction de la Revue Webbia et à l'Institut Botanique de l'Université de Firenze d'avoir si aimablement accepté et pris en charge la publication de ce mémoire.

\section{ChAPITRE PREMIER}

\section{SITUATION GEOGRAPHIQUE ET OROGRAPHIQUE DU BAER-BASSIT ET DU DJEBEL ALAOUITE}

\section{Situation géographique}

Les deux régions naturelles que nous nous proposons d'étudier dans ce mémoire, le Baer-Bassit et le Djebel Alaouite (ou Djebel Ansarié ou Nussairié), sont situées dans le Nord-Ouest de la Syrie, sur le bord occidental de la péninsule Arabique.

Dans son ensemble, ce bord est haut et montagneux et contraste avec le reste de la péninsule qui est formé de plateaux et de plaines horizontaux. Cette horizontalité est due à la présence 
d'un socle granitique rigide; ce socle se dégage an-dessus de la Mer Rouge et du Golfe d'Akaba et se trouve recouvert çà et là par des entablements basaltiques. Mais il plonge vers le $\mathrm{N}$-E et le granite s'enfonce dans cette direction, sous les grès de Nubie et le sédimentaire marin : Jurassique, Crétacé et Tertiaire. Il ne paraît plus dans le $\mathrm{N}-\mathrm{E}$ et le $\mathrm{N}$ de la péninsule, bien qu'il s'y manifeste encore par des grès quartzitiques. Dans sa partie Nord, la bord occidental de la péninsule Arabique est marqué par le sillon du golfe d'Akaba et de la Mer Morte, puis par la Méditerranée.

Le sillon du golfe d'Akaba et de la Mer Morte sépare la péninsule Arabique de la presqu'ile de Sinai, puis se prolonge audelà des lacs de Tibériade et de Houlé jusqu'au Taurus en Turquie, par une série de fossés et de dépressions qui suivent le rivage oriental de la Méditerranée à une quarantaine de kilomètres de distance, ce sont les vallées de la Bekaa au Liban, du Ghab (ou Gharb ou Ghaab) dans le N.W. de la Syrie, entre le Djebel Alaouite et le Djebel Zawié, de l'Amouk et du Kara Sou en Turquie. Cette suite de dépressions fend en long le bord méditerranéen de la péninsule Arabique.

De part et d'autre de la pointe du sillon de la Mer Morte et de la ligne de dépressions qui le prolonge jusqu'au Taurus, s'élèvent de larges massifs juxtaposés presque bout à bout. Ce sont: les monts de Judée, le Liban et 1'Anti-Liban, le Djebel Alaouite et le Djebel Zawié, le Baer et le Bassit et, enfin, l'Amanus et le Kurddagh syrien. Ces massifs constituent une barrière montagneuse N.S. Celle-ci rencontre dans la région de Marach en Turquie, les chaines du Taurus disposées transversalement: WSW-ENE.

Ces massifs et ces dépressions constituent un tout qui représente l'un des traits marquants de l'écorce terrestre dans cette partie du globe.

D'une extrêmité à l'autre, la structure géologique conserve un même caractère. Des mouvements verticaux, le long de grandes failles et de flexures, ont joué un rôle prédominant dans la genèse du relief. Des failles subordonnées découpent une mosaïque de blocs de diverses tailles. Et une certaine simplicité et homogénéité règne dans son développement stratigraphique. 

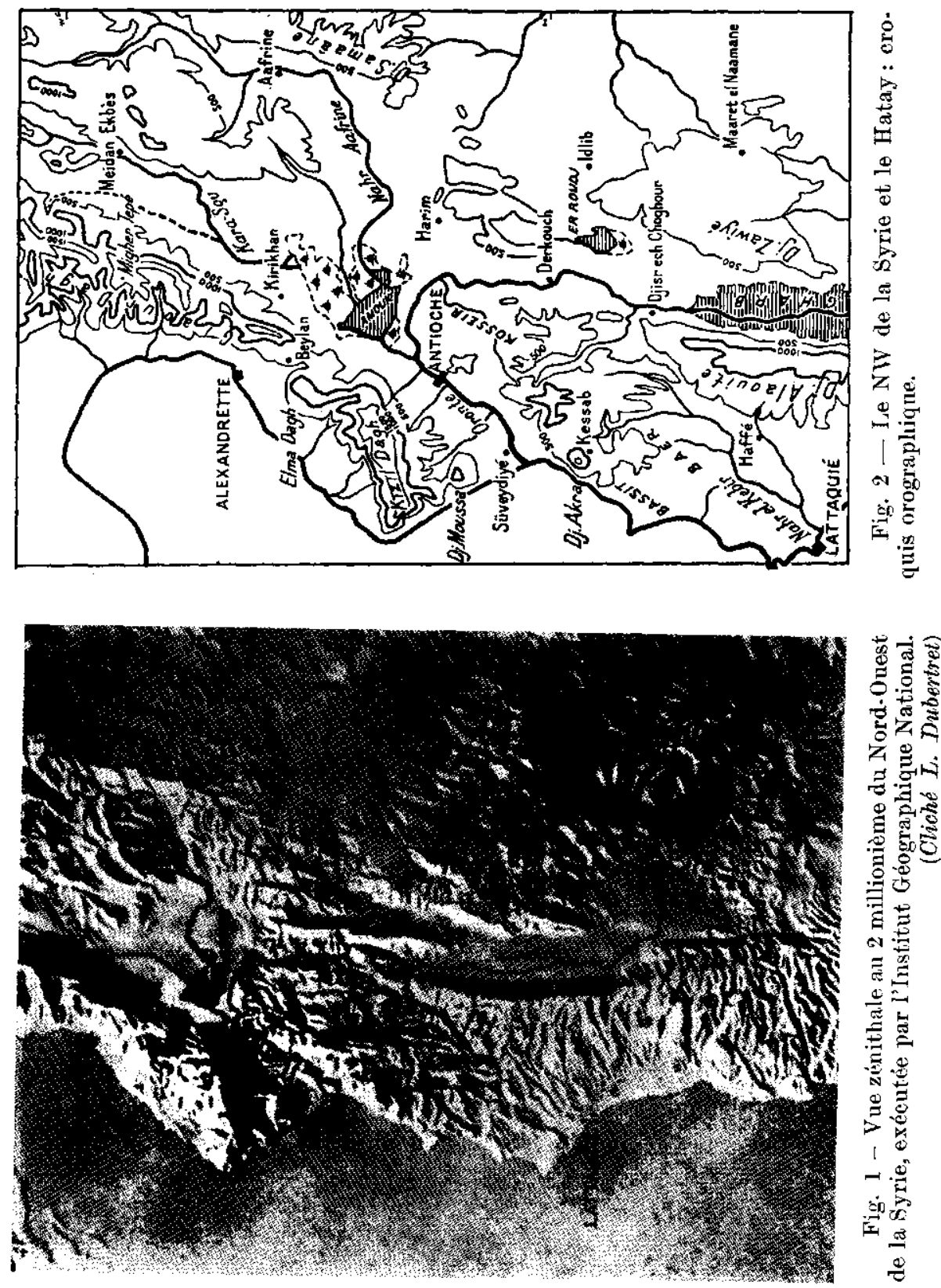
La structure profonde se révèle bien dans les massifs côtiers. La voûte de Judée, peu prononcée, n'est entamée que jusqu'dे la base du Crétacé. Mais le Liban, l'Anti-Liban et le Djebel Alaouite, beancoup plus vigoureux, ont subi un large décapage, qui a mis à nu le Jurassique comme on le verra plus loin ; quelques vallées entaillent celui-ci jusqu'à la base du Jurassique moyen. Les séries découvertes sont essentiellement calcaires, cependant une discontinuité stratigraphique sépare le Jurassique du Crétacé, représentée par des sédiments détritiques quartziques et des basaltes interstratifiés.

Au Nord de la ville de Lattaquié en Syrie, et plus exactement au Nord d'une ligne remontant le cours du Nahr el Kebir de Lattaquié vers le NNE, apparaissent des roches nouvelles, roches inconnues ailleurs en Syrie ou sur la péninsule Arabique: les roches vertes. Elles constituent en Syrie, les deux districts du Baer et du Bassit. Ces roches se poursuivent en Turquie jusqu'au Taurus; elles sont communes dans les chaînes tauriques. Elles constituent également le massif méridional de l'Ile de Chypre, le Troodos.

\section{Paléogéographie}

Le Djebel Alaouite (ou Ansarié ou Nussairié) est un prolongement du Liban. Ses terrains, comme ceux de ce dernier, sont jurassiques, crétacés, tertiaires et quaternaires. Ils correspondent à deux grandes transgressions marines, qui noient complètement le pays, puis aux épisodes de l'immersion jusqu'à la réalisation des reliefs actuels.

La première transgression est jurassique. La deuxième transgression est crétacée; quelques rares poudingues témoignent qu'elle a été séparée de la précédente, au moins localement par une émersion à la fin du Jurassique.

Au Crétacé supérieur et au Nord du Djebel Alaouite, apparait un phénomène nouveau; c'est la mise en place des roches vertes sous forme de nappes éruptives ou coulées sous-marines qui font ainsi intrusion dans la mer et s'enflent jusqu'à atteindre des 
milliers de mètres et finalement émergent et forment des îles (DUBERTRET, 1953).

Les îles des roches vertes ont fourni des matériaux détritiques grossiers et fins aux sédiments déposés dans leur voisinage.

A partir de la fin de l'Eocène moyen, la mer se retire progressivement. Les reliefs qui pouvaient exister jusque là devaient être plats au point de ne pas provoquer d'érosion intense. Ces conditions ont changé à la fin du Nummulitique et c'est au Miocène que l'orogénèse commence véritablement et les reliefs ont alors pris de la vigueur et à peu près leur disposition actuelle.

A la fin du Miocène, les contrastes du relief s'accusent, les blocs montagneux s'élèvent, tandis que les fossés s'affaissent. Le volcanisme est toujours intense. L'érosion entame les massifs de plus en plus profondément.

La fin $\mathrm{du}$ Pliocène se marque par une nouvelle phase orogénique, agissant dans le même sens. Elle isole définitivement le fossé de la Mer Morte.

Le volcanisme a persisté dans le Proche Orient, jusque dans le Quaternaire et l'homme a dû assister à l'épanchement des dernières nappes basaltiques.

L'érosion s'est poursuivie jusqu'à réaliser le paysage actuel.

\section{Orographie}

\subsection{Djebel Alaouite}

Le Djebel Alaouite est un horst essentiellement constitué de calcaires jurassiques et crétacés et situé entre le fossé du Ghab où coule l'Oronte et la Mer Méditerranée. Il est orienté N-S, parallèlement à la côte. Long de $120 \mathrm{~km}$, large de $22 \mathrm{~km}$, il culmine aux environs de $1580 \mathrm{~m}$ au-dessus du Ghab, s'abaisse doucement vers la mer jusqu'à 250-500 m puis s'efface en flexures devant de basses collines néogènes et les plaines côtières quaternaires. Le Nahr el Kebir de Lattaquié et le Nahr el Abiad le délimitent au NW et au N.

Sur son versant occidental, les calcaires crétacés montent brusquement puis ils se replient et continuent une ascension 


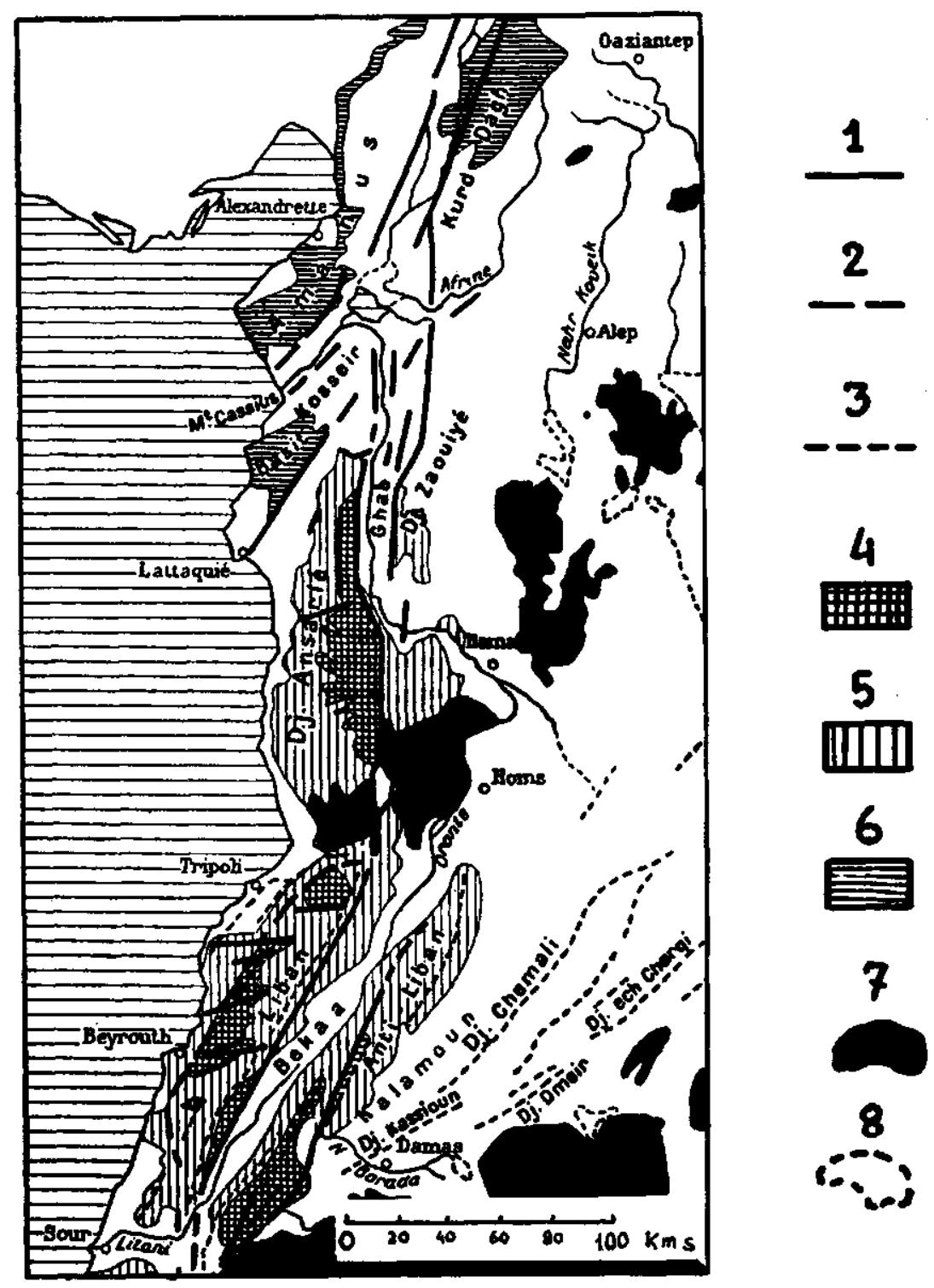

Fig. 3 - La Syrie occidentale et le Liban (croquis structurale). 1 : faille. 2 : flexure. 3 : axe anticlinal. 4 : calcaires jurassiques. 5 : calcaires crétaćés. 6 : roohes vertes crétacées. 7 : coulées basaltiques plio-quaternaires. $8:$ dépressions fermées. 
beaucoup plus douce jusqu'aux crêtes; celles-ci suivent de près la faille orientale dite de Djisr ech Choghour. La ligne des crêtes, très régulière, monte de $1000 \mathrm{~m}$ dans le sud à $1580 \mathrm{~m}$ dans le Nord (Nabi Matta). A ses pieds le Ghab est à $170 \mathrm{~m}$. Sur $15 \mathrm{~km}$, la partie occidentale est constituée par des calcaires crétacés, turoniens et cénomaniens.

Vers le versant oriental, les calcaires jurassiques montent en flexures et portent les sommets $(1583 \mathrm{~m})$; ils constituent un horst étroit, dominant de peu le Crétacé. Ils sont recouverts normalement dans le sud du Djebel Alaouite par la carapace crétacée doucement relevée vers l'Est.

Le Djebel Alaouite s'abaisse doucement du Nord vers le Sud jusqu'à son effacement, créant ainsi une trouée (trouée de Homs-Tripoli) avec la chaîne du Liban.

Notre étude correspond à la partie Nord de la Montagne des Alaouites.

\subsection{Baer-Bassit}

Les deux districts du Baer et du Bassit constituent un pays essentiellement montagneux, de traits confus, s'élevant insensiblement depuis le niveau de la mer jusqu'à $1000 \mathrm{~m}$ d'altitude environ. Le Bassit, le long de la côte, est séparé du Baer, à l'intérieur, par la route Lattaquié-Antioche. Ces deux districts sont le domaine des roches vertes en Syrie.

Au Nord de ces districts se dégage le Djebel Akra (Mont Cassius), cône calcaire jurassique et crétacé s'élevant immédiatement au-dessus de la côte jusqu'à $1725 \mathrm{~m}$ d'altitude.

Depuis la ville d'Antioche jusqu'au Djebel Akra, les roches vertes percent çà et là, capricieusement, à travers une couverture sédimentaire crétacée supérieure et tertiaire. Puis entre le Djebel Akra et le Nahr Kanndil, elles apparaissent largement dénudées, sur une aire de $260 \mathrm{~km}^{2}$, comprenant les districts du Baer et du Bassit. Toutes les parties du complexe des roches vertes sont là également représentées, mais extrêmement morcelées. Les péridotites pyroxéniques constituent les arêtes vives du paysage, tandis que les gabbros, dolérites et pillow-lavas se cantonnent dans les creux. 
Les roches vertes s'enfoncent au Sud du Nahr Kanndil sous une couverture de Cretacé Supérieur et de Nummulitique, qui forme de basses collines marneuses et une plaine: le Sahel de Lattaquié. Mais on les voit s'avancer le long du Nahr el Kébir jusqu'à proximité de Lattaquié et elles constituent le sous-sol d'une partie de la ville.

\section{Chapitre DeuXi亡̀me}

\section{CARACTERISTIQUES DU MILIEU DANS LE BAER-BASSIT ET LE DJEBEL ALAOUITE}

\section{I - LE CLIMAT}

\section{Classification biogéographique des climats - "le bioclimat"}

Plusienrs classifications des climats du Monde ont été proposées par de nombreux auteurs, mais aucune d'elles ne s'est révélée pleinement satisfaisante; e'est sans doute parce que ces classifications ont voulu être générales et valables pour toutes les disciplines. En effet, des classifications ayant cette qualité d'universalité sont impossibles, parce que les points de vue des diverses disciplines scientifiques ne sont pas les mêmes.

Dans "Projet d'une classification biogéographique des climats, 1954 ", L. EMBERGER écrit : Il y a bien des manières de classer les climats : météorologistes et physiciens, par exemple, ayant d'autres exigences que les biologistes, recherchent les critères dans les phénomènes cosmiques (répartition des pressions, circulation atmosphériques, etc.) de l'atmosphère; leurs classifications reposent sur des considérations dynamiques. Les biogéographes, au contraire, ont besoin de connaître exactement les qualités statiques des climats et les mettent au premier rang. Par exemple, la pression atmosphérique, si importante aux yeux des météorologistes et physiciens pour différencier certains climats, $n$ 'a aucune importance pour les biologistes. Les expériences ont montré que l'influence des pressions sur les plantes ne commencent à se manifester qu'à des grandeurs qui n'existent qu'aux plus hautes 
altitudes du globe, auxquelles la vie végétale supérieure est impossible pour d'autres raisons. De plus, il importe peu aux végétaux l'origine de la pluie : ce qui les intéresse, e'est la quantité d'eau qui tombe annuellement et sa répartition sainsonnière.

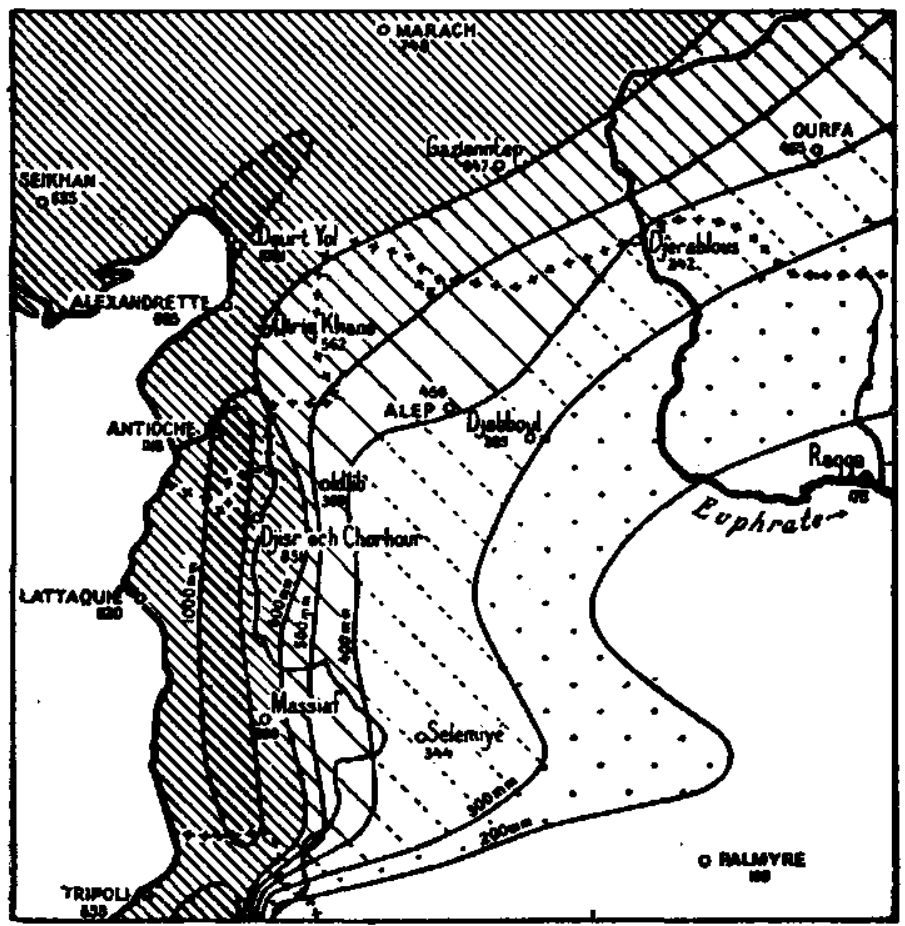

Fig. 4 - Carte pluviométrique $d u \mathrm{NW}$ de la Syrie et des régions attenantes de la Turquie. La succession des isohyètes révèle la rapide transition de la zone côtière humide aux steppes et déserts de l'intérieur.

Les biogéographes, écologistes, etc., doivent donc chercher une classification des climats adaptée à leurs besoins. Ce qui les intéresse, c'est le "bioclimat ".

L. EMrerger a proposé une classification des bioclimats généraux qui se partagent le monde au Congrès International de Botanique de Paris (Juillet 1954).

Dans cette classification, le climat méditerranéen, qui nous intéresse plus particulièrement dans cette étude, est défini comme 
un climat extra-tropical, à photopériodisme quotidien et saisonnier, à pluviosité concentrée sur les saisons froides ou relativement froides de l'année et à saison sèche.

Le trait saillant du climat méditerranéen pour le phytogéographe est la sécheresse estivale qui coincide avec la période de maximum thermique. Elle domine la végétation; les autres caractères viennent en second lieu. Cette sécheresse peut être.plus ou moins accusée.

L. Emberger reconnaît dans le climat méditerranéen les formes suivantes (cours professé à l'Institut Botanique, Montpellier 1961) :

$\begin{array}{l:l}\text { - Climat méditerranéen saharien } \\ \text { - } & \text { aride } \\ \text { - } & \text { semi-aride } \\ \text { - } & \text { sub-humide } \\ \text { humide }\end{array}$

Chacun de ces climats peut, à son tour, être subdivisé en plusieurs variantes, snivant la moyenne des minima du mois le plus froid.

Pour définir ces formes du climat méditerranéen, EMrERGER utilise un Quotient pluviothermique (1930) qui exprime la sécheresse du climat à l'aide de la pluviosité, des températures et de l'évaporation. Il est exprimé dans un système d'axe de coordonnées en combinaison avec (m), la moyenne des minima du mois le plus froid.

Ce quotient est :

$$
Q=\frac{P}{2\left[\frac{\mathbf{M}+\mathbf{m}}{2} \times(M-m)\right]} \times 100=\frac{P}{M^{2}-m^{2}} \times 100
$$

P : Pluviosité moyenne annuelle

M. : Moyenne des maxima du mois le plus chaud

m : Moyenne des minima du mois le plus froid

En partant du zéro absolu $\left(+273^{\circ}\right)$ la formule devient :

$$
Q=\frac{P}{\frac{(M+m)}{2}(M-m)} \times 1000=\frac{2.000 P}{M^{2}-m^{2}}
$$


Cette combination de $(Q)$ et de $(m)$ est réalisée à l'aide d'un système de coordonnées en portant sur l'ordonnée $Q$ et sur l'abcisse (m).

(m) permet de subdiviser les formes du climat méditerranéen en variantes chaude, tempérée, froide et très froide.

$m \geq 7^{\circ}$ caractérise la variante chaude; à ces températures, il n'y a pas

$\mathbf{7 0}^{\circ}>\mathrm{m}>\mathbf{3}^{\circ}$

$3^{\circ}>\mathrm{m}>0^{\circ}$ de gelées ;

$\mathbf{m}<\mathbf{0}^{\mathbf{0}}$

\author{
tempérée; il y a quelques gelées ; \\ froide; gelées assez fréquentes ; \\ très froide; gelées durant de longues \\ périodes.
}

Un autre perfectionnement a été introduit récemment par EMberger en faisant intervenir la pluviosité estivale PE qui avait été négligée auparavant.

La pluviosité estivale est une notion très importante pour les pays méditerranéens. Elle a une grande influence sur la végétation et, en matière forestière, elle doit être prise en considération dans les reboisements, l'introduction des essences étrangères et la régénération naturelle des peuplements. La pluviosité estivale permet, en outre, de différencier des stations ayant le même quotient pluviothermique, mais ayant des pluviosités estivales différentes. Elle va nous permettre de différencier le climat et les étages bioclimatiques de la Méditerranée Orientale des climats et des étages bioclimatiques correspondants de la Méditerranée Occidentale.

Cette formule utilise donc des données météorologiques simples, mais ayant une grande signification biologique.

La moyenne des minima du mois le plus froid $(m)$ et la moyenne des maxima du mois le plus chaud (M) sont très importantes, car c'est entre ces deux limites extrêmes que se déroule la vie végétale, ou plus exactement, la vie végétale se déroule entre $\frac{M+m}{2}$. Cette moyenne n'échappe pas aux défauts de toutes les moyennes, mais elle est corrigée par la différence $(M-m)$ et par $m$.

La différence entre la moyenne des maxima du mois le plus chaud (M) et la moyenne des minima du mois le plus froid (m) exprime, d'une façon indirecte, dans cette formule, l'évaporation et est appellée l'aplitude extrême. 
Ce quotient pluviothermique permet done d'exprimer toutes les formes d'associations que peuvent réaliser les facteurs clima: tiques les plus importants pour la végétation. Il rend compte de la sécheresse d'un pays, et d'une manière générale, exprime la résultante utile du climat pour la végétation. Ce quotient est d'autant plus grand que le pays est plus humide.

Cependant, ce quotient est insuffisant à lui seul et ne permet pas d'expliquer tout. EMBErger le corrige en tenant compte de la moyenne des minima du mois le plus froid (m) qui joue un rôle aussi important que les autres facteurs.

\section{Bioclimats du Baer-Bassit et du Djebel Alaouite}

Pour élucider les bioclimats du Baer-Bassit et du Djebel Alaonite, nous avons choisi des stations situées sur le littoral et dans le Djebel Alaouite. Ce choix n'a pas été facile, étant donné le nombre très restreint de stations météorologiques dans la région. En outre, ces stations, même quand elles existent, sont très récentes.

Nous avons fait, pour chaque station, deux sortes de diagramme : 1) un premier diagramme combinant la moyenne mensuelle des pluies et les moyennes mensuelles des températures maximales et minimales ; 2) un deuxième diagramme montrant la répartition saisonnière des pluies. Nous avons calculé pour chaque station le quotient pluviothermique $Q\left({ }^{1}\right)$ et la pluviosité estivale PE.

Tableau broclimatique

\begin{tabular}{|c|c|c|c|c|c|c|}
\hline & $\underset{\text { m }}{\text { Alt. }}$ & $\underset{\mathbf{m m}}{\mathbf{P}}$ & $\mathbf{M}$ & $\mathbf{m}$ & $\mathbf{Q}$ & $\begin{array}{c}\mathbf{P E} \\
\mathbf{m m}\end{array}$ \\
\hline Lattaquié & 50 & 805 & 31 & 7 & 115 & 8 \\
\hline Bouka & 100 & 837 & 31,8 & 8,2 & 121 & 12 \\
\hline Haffé & 350 & 891,2 & 28 & 5 & 133,8 & 40,8 \\
\hline Kassab & 900 & 1050 & 27,2 & 3 & 150,6 & 60 \\
\hline Slenfé & 1100 & 1046 & 26,2 & 1,6 & 149,5 & 30 \\
\hline
\end{tabular}

(1) Le quotient pluviométrique a été calculé d'après la formule $Q=\frac{2.000 \mathrm{P}}{\mathrm{M}^{2}-\mathrm{m}^{2}}$ 
Le climat de la région littorale peut être représenté par celui que nous indiquent les deux stations littorales de Lattaquié et Bouka; celui des basses montagnes par celui de la station de Haffé; celui des montagnes moyennes par celui des stations de Kassab et Slenfé.

Analysons ces différents diagrammes et ce tableau bioclimatique :

1. Diagrammes de Lattaquié et de Bouka.

Les quatre diagrammes 1,2 , 1 bis et 2 bis et le tableau bioclimatique nous permettent de dégager les caractéristiques suivantes du climat de la région littorale :

a) la pluviosité moyenne annuelle est de l'ordre de $800 \mathrm{~mm}$ environ (805 $\mathrm{mm}$ pour Lattaquié ; $837 \mathrm{~mm}$ pour Bouka).

b) La présence d'une saison sèche, très marquée, coincidant avec une période de maximum thermique. Cette saison sèche est l'été, mais elle déborde également sur l'automne; le mois de septembre est aussi sec que les mois de l'été. La pluviosité estivale est pratiquement nulle; elle est de $12 \mathrm{~mm}$ pour Bouka et $8 \mathrm{~mm}$ pour Lattaquié.

c) La pluviosité est répartie sur les saisons froides ou relativement froides : hiver, printemps, automne. Mais, elle est surtout concentrée sur l'hiver; plus de la moitié de la pluviosité annuelle tombe en hiver (473 $\mathrm{mm}$ pour Bouka, $502 \mathrm{~mm}$ pour Lattaquié).

d) La moyenne des minima du mois le plus froid (m) est nettement audessus de zéro; elle est de $7^{\circ}$ pour Lattaquié et 802 pour Bouka. Le quotient pluviothermique d'EMBERGER est de 121 pour Bouka, et 115 pour Lattaquié). Le climat de la région littorale est un climat méditerranéen subhumide chaud.

2. Du diagramme (4) de Haffé (situé entre 350 et $400 \mathrm{~m}$ d'altitude dans le Djebel Alaouite) nous dégageons les caractéristiques climatiques suivantes :

2.1. La pluviosité moyenne annuelle s'élève à $891,23 \mathrm{~mm}$.

2.2. L'allure du diagramme ressemble à celle des diagrammes 1bis et 2bis de Lattaquié et Bouka. Le creux estival est toujours bien marqué; mais à Haffé, la pluviosité estivale est légèrement 
LA Vtgétation DU BaER-Basgit at DU DJ. ALAOUITE (SYRIE) 491

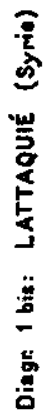

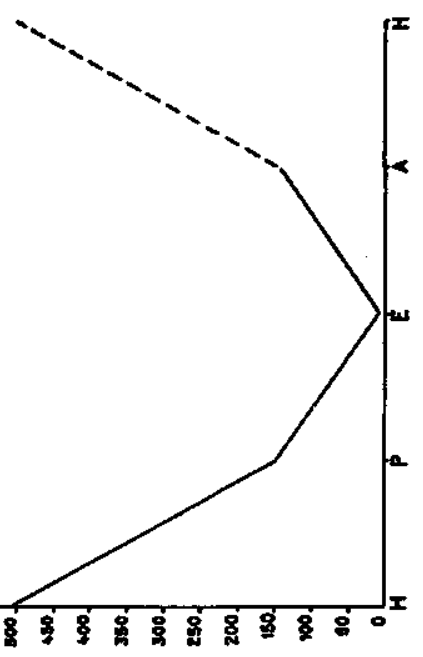

wis us ettrotants

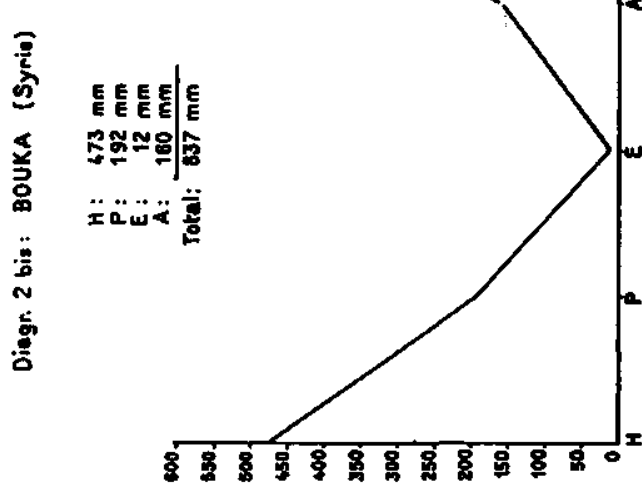

wee us apropantd

Thes squbop uo soungespduses

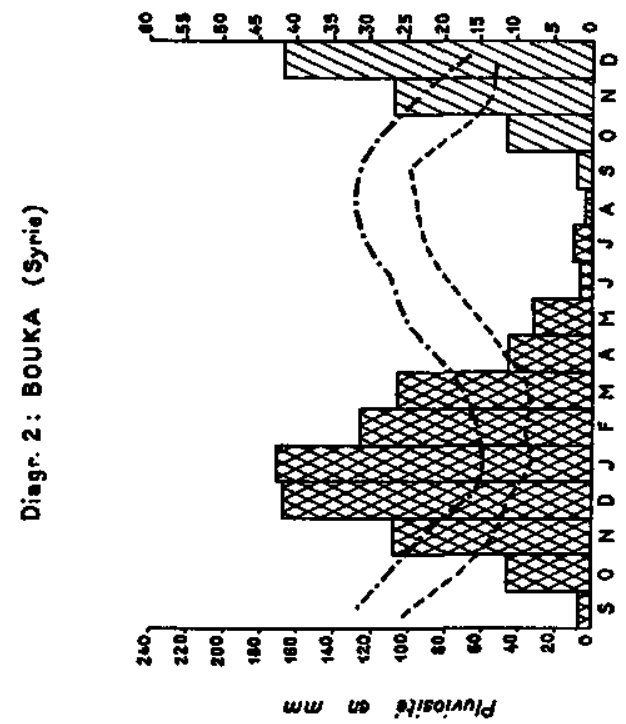

we the Misolanld

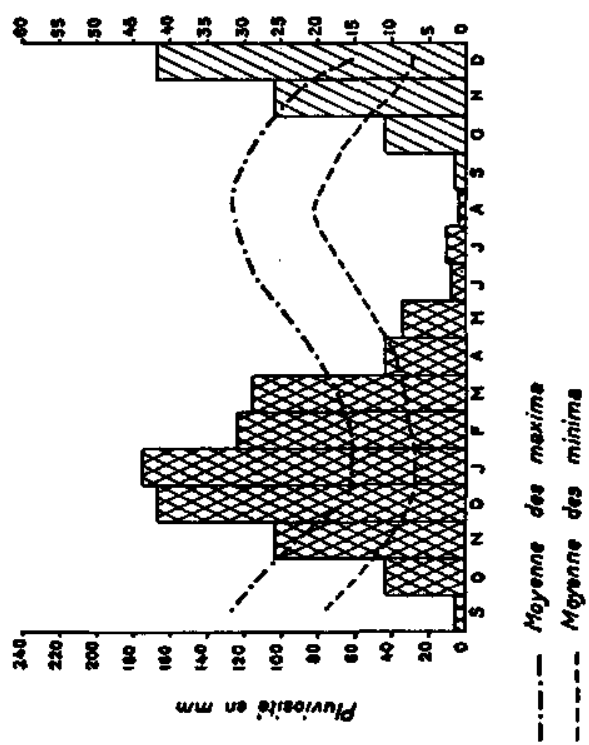


plus élevée ; elle est de l'ordre de $\mathbf{4 0 , 8} \mathrm{mm}$. La répartition saisonnière des pluies est la même que sur le littoral.

2.3. Le quotient pluviothermique est égal à 133,8. La moyenne des minima du mois le plus froid est égale à 5 . Le climat de Haffé est à la limite du climat méditerranéen humide tempéré et subhumide tempéré.

3. Des diagrammes 5,6 et 6 bis de Kassab (Baer-Bassit) et de Slenfé (Djebel Alaouite) nous dégageons les caractéristiques climatiques suivantes : $1000 \mathrm{~mm}$.

3.1. La pluviosité moyenne annuelle est supérieure à

3.2. Le creux estival est toujours très marqué et coincide toujours avec une période de maximum thermique. La pluviosité estivale est légèrement plus élevée que celle des stations littorales ( $\mathrm{PE}=36 \mathrm{~mm}$ pour Slenfé et $60 \mathrm{~mm}$ pour Kassab).

3.3. La répartition saisonnière des pluies est légèrement différente de celle des stations littorales. Les pluies printanières sont plus fortes; elles sont de l'ordre de $356 \mathrm{~mm}$ pour Slenfé et de $320,3 \mathrm{~mm}$ pour Kassab. Les pluies d'hiver sont presque identiques à celles des stations littorales.

3.4. Le quotient pluviothermique est élevé. Il est égal à 150,6 pour Kassab et à 149,5 pour Slenfé. La moyenne des minima du mois le plus froid $(\mathrm{m})$ est égal à 3 pour Kassab et 1,6 pour Slenfé. Le climat de Kassab et de Slenfé est un climat méditerranéen humide froid.

A partir de Slenfé, on commence à sentir le climat perhumide froid. L'absence de stations météorologiques ne nous permet pas d'élucider ce point de vue, mais la nature et la composition de la végétation entre 1200 et $1580 \mathrm{~m}$ d'altitude (Cedretum, Abietum) nous indiquent que nous sommes sous le climat méditerranéen perhumide froid.

En résumé, le climat qui règne dans le Baer-Bassit et le Djebel Alaouite est un climat méditerranéen. Il se présente sous trois formes et plusieurs variantes :

- le climat méditerranéen subhumide chaud;

- le climat méditerranéen humide, tempéré et froid;

- le climat méditerranéen perhumide froid. 
LA végétation DU BaER-Bassit ET DU DJ. aLAOUtTe (SYRIE) 493

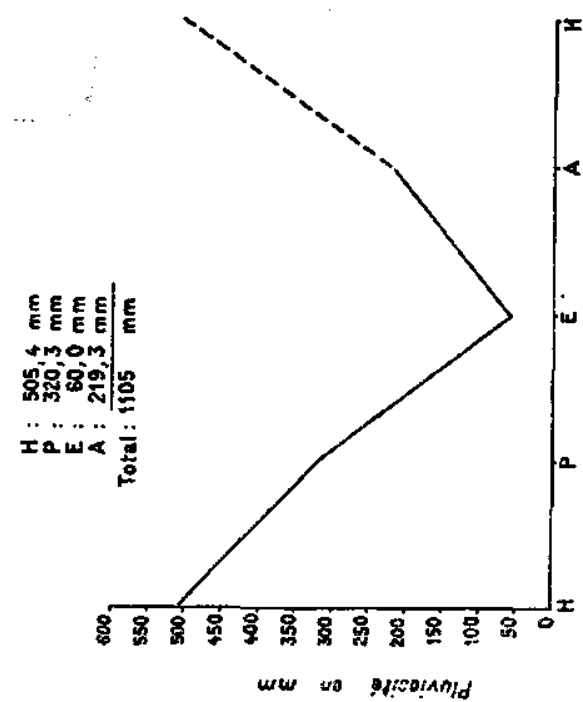

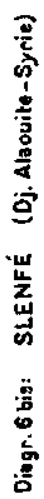

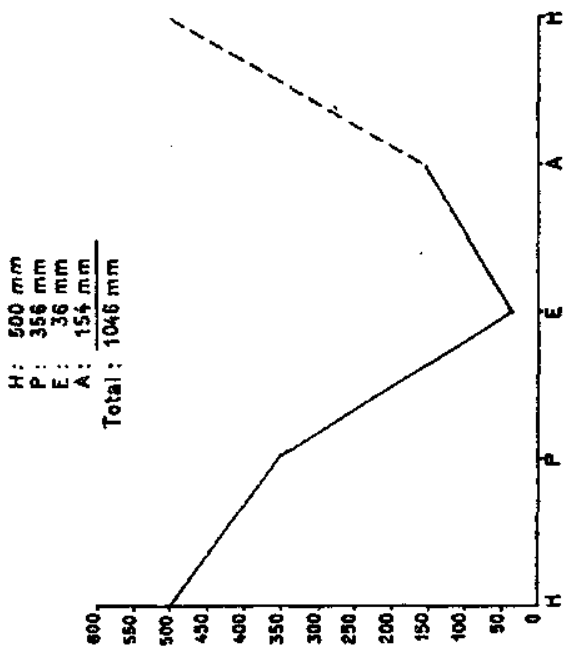

wiw wo efisoinand

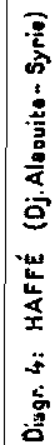
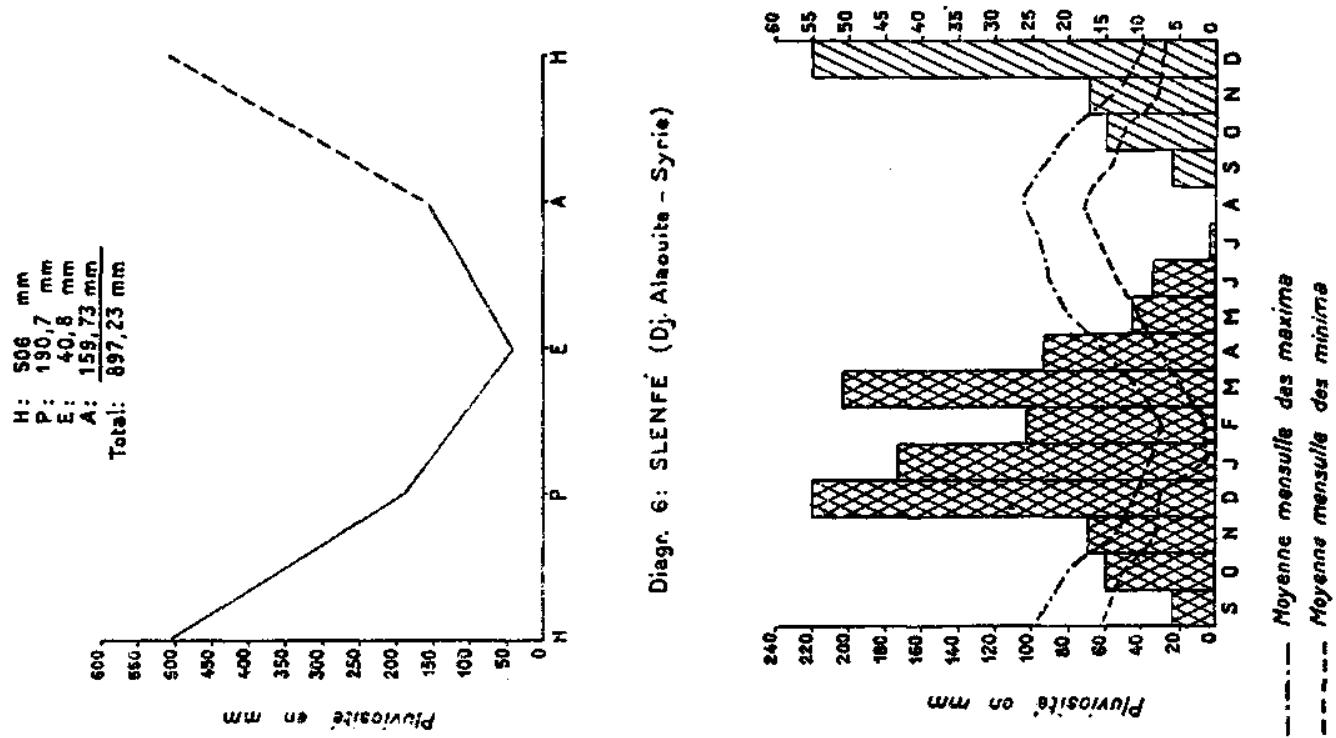
Certains auteurs (Gaussen, 1954) considérent le climat des zones où poussent les sapins circumméditerranéens, les cèdres, les divers pins laricios... dans les montagnes côtières de Turquie, Syrie, Liban, Chypre, comme n'appartenant plus au climat méditerranéen. Nous ne partageons pas avec ces auteurs cette opinion pour les raisons suivantes:

- les courbes thermiques de la station de Slenfé, qui est située à $1100 \mathrm{~m}$ d'altitude, montrent clairement qu'elles sont les mêmes que celles des stations littorales de Bouka et de Lattaquié (voir Diagr. 1, 2 et 6 ) avec un certain décallage dû à l'altitude.

- La courbe pluviométrique de la station de Slenfé a la même allure que celle des stations littorales avec, seulement, une pluviosité printanière plus élevée.

- La flore et la végétation sont typiquement méditerranéennes : on y trouve : Pistacia palaestina, Phillyrea media, Quercus infectoria, Quercus calliprinos, Styrax officinalis, Cistus villosus, Cistus salviaefolius, etc. La végétation est soumise au rythme saisonnier méditerranéen.

Beaucoup d'auteurs (Gombault, etc.) quand ils parlent du climat ou de la végétation des hautes montagnes du ProcheOrient, emploient les termes "subalpin et alpin ". Or ces termes prêtent à confusion et sont imprécis et doivent être écartés pour éviter des parallélismes injustifiés avec le climat des hautes montagnes de l'Europe tempérée.

\section{Comparaison du régime climatique du Baer-Bassit et du Djebel Alaouite avec celni de la Méditerrancée occidentale}

Nons avons vu en détail les caractéristiques des climats méditerranéens du Baer-Bassit et du Djebel Alaouite et remarqué que leur earactéristique principale était la présence d'une saison sèche très prononcée. Il serait très utile, de comparer ces climats de la Méditerranée orientale avec les climats analogues de la Méditerranée occidentale. Cette comparaison nous permettra de mieux situer les climats de la région que nous étudions à l'intérieur du climat méditerranéen général.

Nous avons choisi pour cette comparaison deux stations lit- 
torales ayant la même pluviosité annuelle, mais dont l'une est située sur la Méditerranée orientale (station de Lattaquié) et l'autre sur la Méditerranée occidentale (station d'Antibes en France). La pluviosité annuelle est de $805 \mathrm{~mm}$ à Lattaquié et $808 \mathrm{~mm}$ à Antibes.

La comparaison des diagrammes 7 (Antibes) et 1bis (Lattaquié) nous permet de tirer les conclusions suivantes : $\left(^{(1)}\right.$

\begin{tabular}{|c|c|c|c|}
\hline & Antiber & & Lattaquié \\
\hline $\begin{array}{l}\mathbf{H}: \\
\mathbf{P}: \\
\mathbf{E}: \\
\mathbf{A}:\end{array}$ & $\begin{array}{r}237 \mathrm{~mm} \\
199 \mathrm{~mm} \\
76 \mathrm{~mm} \\
296 \mathrm{~mm}\end{array}$ & $\begin{array}{l}\text { H : } \\
\mathbf{P}: \\
\mathbf{E}: \\
\mathbf{A}:\end{array}$ & $\begin{array}{r}502 \mathrm{~mm} \\
149 \mathrm{~mm} \\
8 \mathrm{~mm} \\
146 \mathrm{~mm}\end{array}$ \\
\hline Total & $808 \mathrm{~mm}$ & Total & $805 \mathrm{~mm}$ \\
\hline
\end{tabular}

A Lattaquié, les pluies sont surtout concentrées sur l'hiver et l'été est extrêmement sec. La pluviosité estivale est pratiquement nulle à Lattaquié, alors qu'elle s'élève à $76 \mathrm{~mm}$ à Antibes.

Diagr.7: ANTIBES (France)

Diagn 8: PERPIGNAN (Pyréntes Orientolos- Frante)
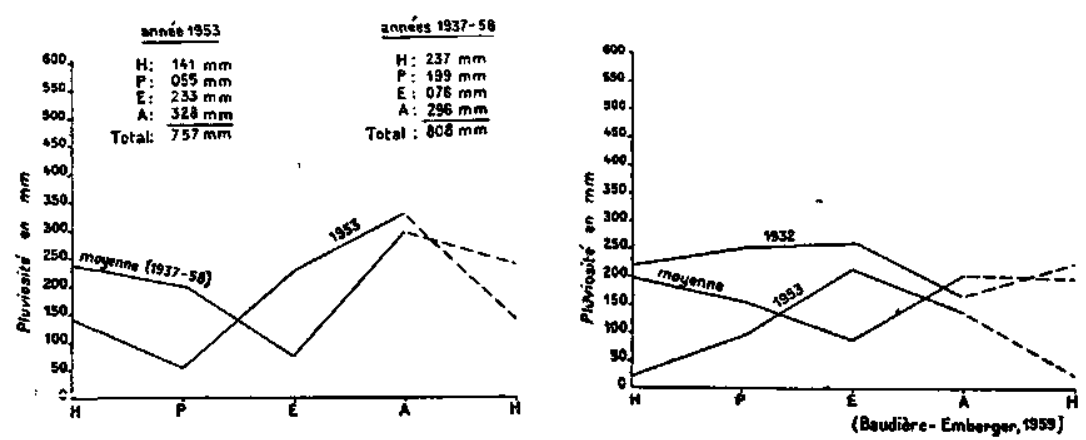

Les pluies d'automne sont importantes à Antibes et sont supérieures aux pluies d'hiver.

A. BAUDIÈRE et L. EMBERger (1959) ont montré qu'au fur et à mesure que l'on s'éloigne des côtes méditerranéennes de la

(1) Le laps de temps choisi pour les denx stations n'est pas le même. Il ne semble pas qu'il y ait inconvénient majeur d'avoir agi ainsi, puisqu'il ne s'agit pas de comparer des quantités de pluies, mais des régimes pluviométriques. 
France, la pluviosité estivale va en croissant, de sorte que certaines années, la courbe peut présenter une convexité estivale au lieu d'un creux et ceci même pour des stations typiquement méditerranéennes. Lorsque la fréquence d'un tel régime l'emporte sur les années à pluviosité estivale méditerranéenne (courbe concave en été) on n'est plus sous le climat méditerranéen, mais sous un climat de transition.

Même à Antibes (station très méditerranéenne), on observe certaines années (1953) à climat non méditerranéen et où la courbe pluviométrique présente une convexité estivale an lieu d'une concavité.

$$
\begin{array}{rr}
\multicolumn{2}{c}{\text { année } 1953} \\
\text { H : } & 141 \mathrm{~mm} \\
\text { P : } & 55 \mathrm{~mm} \\
\text { E : } & \mathbf{2 3 3} \mathrm{mm} \\
\text { A : } & \mathbf{3 2 8} \mathrm{mm} \\
\text { Total } & \mathbf{7 5 7} \mathrm{mm}
\end{array}
$$

Le même phénomène a été observé à Perpignan (France) où certaines années (1932 et 1953) la courbe pluviométrique présente une convexité (voir Diagr. 8) et dénote ainsi un régime non méditerranéen. A Perpignan, le régime pluviométrique moyen, calculé

\begin{tabular}{|c|c|c|c|}
\hline \multicolumn{2}{|c|}{ année 1932} & \multicolumn{2}{|c|}{ année 1953} \\
\hline H : & $226 \mathrm{~mm}$ & H : & $22 \mathrm{~mm}$ \\
\hline$P$ : & $248 \mathrm{~mm}$ & P: & $94 \mathrm{~mm}$ \\
\hline $\mathbf{E}$ : & $255 \mathrm{~mm}$ & $\mathrm{E}:$ & $212 \mathrm{~mm}$ \\
\hline A: & $164 \mathrm{~mm}$ & A : & $138 \mathrm{~mm}$ \\
\hline Total & $893 \mathrm{~mm}$ & Total & $466 \mathrm{~mm}$ \\
\hline
\end{tabular}
sur 24 années d'observations (1929-1941 et 1944-1954) est le suivant (BAUDIk̀RE et EMBERGER 1959):

$$
\begin{array}{rr}
\text { H : } & 197 \mathrm{~mm} \\
\text { P : } & 154 \mathrm{~mm} \\
\text { E : } & 89 \mathrm{~mm} \\
\text { A : } & 206 \mathrm{~mm} \\
\text { Total } & 646 \mathrm{~mm}
\end{array}
$$

Le creux estival est ici nettement accusé encore, mais certaines années (1932, 1953), la courbe peut s'inverser (voir diagr. 8): 
Mais ces années à régime non méditerranéen son noyées dans le nombre d'années nettement méditerranéennes. Elles sont pourtant révélatrices d'un état climatique moins stable que dans les stations de la Méditerranée orientale où le régime pluviométrique est toujours méditerranéen.

Le climat d'Antibes diffère de celui de Lattaquié par les caractères suivants :

1) une pluviosité annuelle relativement mieux répartie sur les saisons ;

2) un accroissement net de la pluviosité estivale; le creux estival tend à se combler;

3) le régime climatique de certaines années n'est pas méditerranéen.

L'importance biologique de ces caractères différentiels est évidente. La végétation qui est soumise au climat d'Antibes a une écologie différente de celle soumise au climat de Lattaquié.

\section{Les etages bioelimatiques dans le Baer-Bassit et le Djebel Alaouite}

Lorsqu'on étudie les rapports entre la végétation et le climat d'un pays (pays méditerranéen par exemple), on constate qu'il y a une corrélation entre les unités naturelles de la végétation et les formes du climat de ce pays. Ces unités de végétation auxquelles correspondent des formes de climats caractéristiques sont appellées par EMBERGER des étages bioclimatiques de végétation.

L'étage est défini ici par le climat et la végétation ensemble. C'est donc, une unité ayant ses caractères propres et indépendantes de l'altitude et de la floristique.

C'est ainsi qu'on peut distinguer six étages bioclimatiques méditerranéens correspondant aux six formes du climat méditerranéen :

Etage bioclim. médit. perhumide

\begin{tabular}{|c|c|c|c|}
\hline & 4 & $\#$ & humide \\
\hline " & " & \# & subhumide \\
\hline ) & $"$ & $"$ & semi-aride \\
\hline " & $"$ & $"$ & aride \\
\hline D & : & * & saharien \\
\hline
\end{tabular}

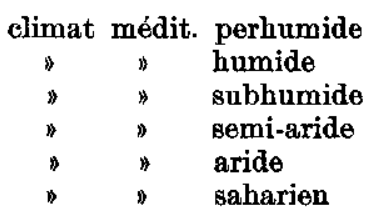


Comme nous l'avons vu pour le climat, ces étages bioclimatiques peuvent eux-mêmes être subdivisés en premier lieu suivant la grandeur de (m), puis d'après la pluviosité estivale et le régime pluviométrique.

Dans le Baer-Bassit et le Djebel Alaouite, on trouve done les étages bioclimatiques méditerranéens suivants :

1) l'étage blioclimatique méditerranéen subhumide chaud de la région littorale;

2) l'étage bioclimatique méditerranéen humide avec ses variantes : tempérée et froide. Cet étage englobe les régions basses et moyennes des montagnes;

3) l'étage bioclimatique méditerranéen perhumide froid qui est l'étage de la partie supérieure du Djebel Alaouite.

La notion d'étage bioclimatique est très utile anx forestiers, agronomes, phytogéographes, etc. Elle permet de comparer des régions fondamentalement différentes par leur flore, mais écologiquement et biologiquement identiques. Ceci est très important pour l'introduction des essences forestières étrangères, les reboisements, le problème de mise en valeur car, à climat égal, les espèces qui constituent des types de végétation sont en principe interchangeables et peuvent être introduites d'un pays à l'autre.

Dans cette étude nous utiliserons cette notion d'étage bioclimatique pour l'étude de l'écologie des essences forestiéres et des associations végétales.

\section{II - LES ROCHES-MERES}

1. Importance de la connaissance des roches-mères pour l'étude de la vegétation dans le Baor-Bassit et le Djebel Alaouite

La connaissance des roches-mères non altérées ou en voie d'altération est très importante pour l'étude de la végétation du Baer-Bassit et du Djebel Alaouite pour les raisons suivantes :

1. La végétation spontanée dans ces régions a été très influencée et modifiée par l'homme depnis des millénaires. Dans beaucoup de périmètres la végétation primitive a complètement disparu et a été remplacée par une végétation secondaire de 
garrigues, maquis ou landes. Le sol climacique a suivi une évolution analogue par dégradation et érosion et la roche-mère (ou le sous-sol géologique), s'est trouvée mise à $\mathrm{nu}$, influençant ainsi directement la végétation.

La végétation secondaire est étroitement liée à la nature physico-chimique de la roche-mère; l'alimentation en eau des végétaux dans ces sols dégradés, par exemple, dépend étroitement des caractéristiques physiques de la roche-mère. Nous avons remarqué en plusieurs stations dans le Djebel Alaouite, que la régénération du Pinus brutia se fait aisément sur les roches-mères marneuses presque nues alors qu'elle est exceptionnelle sur la Terra rossa. En effet, sous un climat à saison très sèche comme le nôtre, et sous l'influence de l'évaporation estivale intense, il se forme sur la Terra rossa une croûte superficielle très sèche et dure, done défavorable à l'alimentation en eau des jeunes semis de pins et à leur développement.

2. La roche-mère a une importance secondaire dans les pays tempérés où le climat est assez fort pour masquer son influence. Dans ces régions, le climat joue un rôle de première importance. C'est ainsi que dans ces régions on a été amené à parler du sol-climax ou sol analogue (PALMANN 1949) qui présente des caractères propres le rendant indépendant de la roche-mère primitive. Ces caractères doivent être imputés à l'action combinée du climat et de la végétation, cette dernière agissant sur la matière minérale par l'intermédiaire de l'humus qu'elle lui incorpore.

Dans le Baer Bassit et le Djebel Alaonite et en général dans les pays méditerranéens où règne un climat à saison sèche et à pluies tombant en averses, le sol reste presque toujours dépendant de la roche-mère qui lui a donné naissance en raison de l'impuissance de ce climat à modifier radicalement le substratum géologique.

3. Même quand il s'agit de sols évolués se rapprochant du sol-climax ou du sol analogue et qui ont pu se conserver sous certaines végétations proches du climax, done très peu modifiées par l'homme, mais provenant de l'évolution de roches-mères différentes (Péridotites pyroxéniques, Terra rossa, marne...), il n'y a que les couches supérieures qui présentent des caractères morphologiques semblables et des propriétés communes dues à l'action combinée d'un même climat et d'une même végétation. Les hori- 
zons plus profonds conservent des caractères propres qui sont très voisins de ceux de la roche-mère, donnant ainsi à ces sols analogues des caractères particuliers.

La végétation arborescente est très sensible à cette différence des couches profondes en raison de ses racines qui vont en profondeur à la recherche de l'eau et des matières minérales. C'est ainsi que les sols formés sur péridotites pyroxéniques, superficiels et retenant très peu l'eau de pluies, sont colonisés par le Pinus brutia, essence peu exigente en eau, alors que les sols formés sur gabbros ou dolérites, plus profonds et retenant mieux l'eau, sont colonisés par le Quercus pseudocerris, essence forestière beaucoup plus exigente en eau.

4. Le Baer-Bassit est un pays essentiellement montagneux. Nous y trouvons beaucoup de sols jeunes en raison de la pente accentuée du terrain qui freine leur évolution. Dans ce cas, la roche-mère présente une importance spéciale vis-à-vis de la végétation arborescente car ces sols jeunes offrent des propriétés voisines de celles de la roche-mère.

5. Dans la Région méditerranéenne en général, et dans le Baer-Bassit et le Djebel Alaouite en particulier, le facteur " eau " domine tous les autres facteurs. La perméabilité des roches-mères à l'eau de pluie constitue un des facteurs les plus importants dans l'installation de la population rurale et la création des villages. La présence de nombreuses sources dans un périmètre donné nous permet de comprendre la disparition de la végétation primitive de ce périmètre, sa dégradation ou son remplacement par des cultures agricoles ou arboricoles. C'est ainsi que dans le Baer et. le Bassit, pays de roches vertes, où existent des péridotites pyroxéniques et des gabbros, diorites et dolérites, un profond contraste existe entre le pays des péridotites pyroxéniques et serpentine, rocailleux, privé de sources, inhabité et le pays des gabbros, dolérites et diorites, aux sols plus doux, plus aimables, riches en sources et parsemés de villages.

L'homme a déboisé dans le Baer et le Bassit les régions où la fôret poussait sur des bons sols, en particulier sur des sols. formés sur argile rouge, marnes, gabbros, dolérites et amphibolites. En outre, la plupart de ces roches sont perméables à l'eau de pluie et entretiennent des sources. 


\section{Les principaux types de roches-mères}

\subsection{LES ROCHES VERTES}

Les roches vertes sont des roches éruptives basiques localisées dans les deux districts du Baer et du Bassit, au Nord de la ville de Lattaquié (Laodicée). Il est bon de signaler que ces roches vertes se poursuivent en territoire ture jusqu'au Taurus et existent également dans l'Ile de Chypre où elles constituent le massif méridional de Troodos. Elles n'existent pas dans le Djebel Alaouite, massif essentiellement calcaire.

Les études de DuberTret (1952) ont conduit à la conclusion que ces roches se trouvent dans leur gisement originel, qu'elles ont été mises en place par des coulées sous-marines, qui se sont répandues sur un substratum incluant des couches maestrichtiennes (Crétacé supérieur). Ces coulées se sont gonflées par intrusion de magma dans leur partie profonde, jusqu'à atteindre 3000-4000 mètres d'épaisseur. En se gonflant, elles ont emergé et se sont trouvées soumises à l'érosion. Les parties restées immergées ont été recouvertes de sédiments détritiques maestrichtiens.

Les divers types de roches associées dans le corps des roches vertes se succèdent dans un ordre déterminé : au-dessus des péridotites pyroxéniques, suivent des gabbros à olivine, puis des gabbros sans olivine et des dolérites, enfin des sakalavites à délits en oreillers (Pillow-lava). Des radiolarites sont associées aux pillows-lavas ou bien les recouvrent.

Du point de vue qui nous intéresse, ces roches-vertes présentent entre elles des propriétés très différentes tant du point de vue pétrographique que physique. Ces propriétés ont une importance primordiale dans l'altération de ces roches, leur pédogénèse et les propriétés physico-chimiques des sols qui en résultent.

De ce point de vue, nous pouvons grouper les roches vertes en 2 groupes : 1) groupe des péridotites pyroxéniques ; 2) groupe des gabbros, diorites et dolérites. 


\subsubsection{Groupe des péridotites pyroxéniques (1er groupe)}

Ce groupe est le plus représenté dans le Baer et le Bassit. Il constitue les massifs du Qizil Dagh syrien, Alkawamii, Qastal Moaf et en général, toutes les parties saillantes de la région.

Leurs constituants sont uniquement des minéraux colorés : olivine, augite, diallage, c'est-à-dire des silicates ferro-magnésiens non alumineux ou alumineux et la serpentine produit de leur altération. Leur cassure montre le plus souvent un fond mat, vert très foncé tirant sur le brun rouge, de serpentine, emballant des paillettes nacrées de diallage. Extérieurement, elles sont rubéfiées.

L'analyse chimique des péridotites pyroxéniques (voir tableau des analyses) a montré que ces roches sont riches en silicates $(40-45 \%)$ et en Magnésie ( $M g O: 27-45 \%)$. Leur teneur en fer $\left(\mathrm{Fe}_{2} \mathrm{O}_{3}: 0,70\right.$ à $5 \% ; \mathrm{FeO}: 2,35$ à $\left.4,75 \%\right)$ est assez appréciable. Par contre, leur teneur en $\mathrm{CaO}$ est assez faible. L'analyse chimique a décelé aussi la présence de traces de Nickel (NiO : 0,20 à $0,30 \%$ ), qui pourraient avoir une action spéciale sur la végétation que nous discuterons plus loin dans le chapitre quatrième.

La décomposition des péridotites pyroxéniques sous l'influence du climat et de la végétation donne des sols siliceux, magnésiens, ferrugineux et paurres en bases $\left(\mathrm{CaO}, \mathrm{Na}_{2} \mathrm{O}\right)$.

Ces roches sont grenues, dures et compactes. Elles sont imperméables à l'eau de pluie qui ruisselle sur leur surface et ne produisent pas de sources.

Les eaux courantes les sculptent vigoreusement taillant en $\mathrm{V}$ leurs vallées en marquant nettement la position des crêtes.

\subsubsection{Groupe des gabbros, diorites et dolérites (2ème gronpe)}

Les roches-mères appartenant à ce groupe sont moins fréquentes que les péridotites pyroxéniques et serpentine dans le Baer et la Bassit. Mais leur présence n'en reste pas moins importante.

Ces roches diffèrent de celles $d u$ groupe précédent par l'association aux minéraux colorés, de minéraux blanes : feldspaths calciques et calco-sodiques, c'est-à-dire mélanges de l'anorthite $\left(\mathrm{CaO}, \mathrm{Al}_{2} \mathrm{O}_{3}, 2 \mathrm{SiO}_{2}\right)$ et de l'albite $\left(\mathrm{Na}_{2} \mathrm{O}, \mathrm{Al}_{2} \mathrm{O}_{3}, 6 \mathrm{SiO}_{2}\right)$.

L'analyse chimique de ces roches (voir tableau) a montré leur 


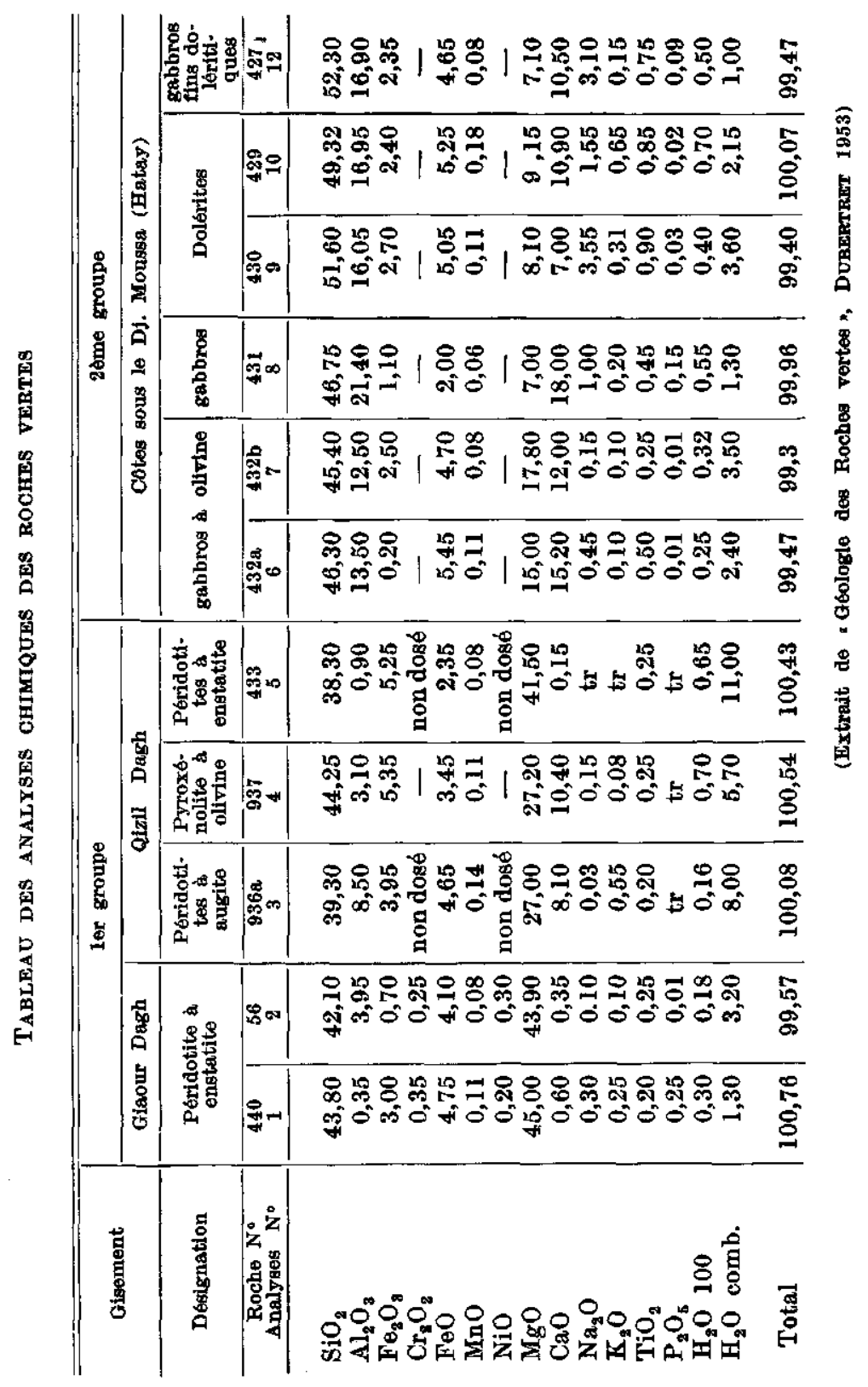


richesse en $\mathrm{SiO}_{2}(45$ à $52 \%)$. Par comparaison avec les roches du premier groupe, ces roches sont plus riches en Alumine $\left(\mathrm{Al}_{2} \mathrm{O}_{3}\right.$ : $12,5$ à $31,40 \%)$, en $\mathrm{CaO}\left(7 \mathrm{à} 18 \%\right.$ ) et en $\mathrm{Na}_{2} \mathrm{O}(0,15 \mathrm{a} 3,55 \%)$; par contre, elles sont moins riches en $\mathrm{MgO}(7,10$ à $17,8 \%$ ) et dépourvues de Nickel. Leur teneur en fer est à peu près la même que celle des roches du premier groupe. Le gabbro de la région de Duz Archach dans le Baer-Bassit a la même composition minéralogique que les gabbros à olivine 432a et $432 \mathrm{~b}$ du Dj. Moussa, mais il est légèrement plus calcique, son plagioclase est un labrador à $90 \%$ d'anorthite.

La décomposition de ces roches sous l'influence du climat et de la végétation donne des sols siliceux, ferrugineux, beaucoup moins magnésiens que les sols du premier groupe, mais plus riches en bases $\left(\mathrm{CaO}, \mathrm{Na}_{2} \mathrm{O}\right)$.

En outre, les gabbros et dolérites sont facilement altérables et donnent des sols profonds. Ils sont perméables à l'eau de pluie et donnent naissance à des sources autour desquelles se sont construits des villages.

\subsection{LES ROCHES-MÈRES CALCAIRES}

Ces roches-mères sont très répandues dans le N.W. de la Syrie et constituent presque à elles seules le Djebel Alaouite et le Djebel Akraa. Ce groupe réunit des roches très variées que nous allons étudier dans ce qui suit :

\subsubsection{Le calcaire compact}

Nous désignons sous le terme "calcaire compact " un ensemble de roches calcaires compactes, très pures, à grain fin, se trouvant en puissants massifs et au-dessus desquels on ne voit jamais apparaître d'autres formations. Ces calcaires appartiennent à une série de formations géologiques variées; ce sont les calcaires jurassiques, crétacés et éocènes du Djebel Akra et de la bordure septentrionale des Alaouites. Ces mêmes calcaires se retrouvent dans le Liban, l'Antiliban, le Djebel Zaouié et le Qalamoun (au N. de Damas). 
Ces roches de calcaire pur se transforment par corrosion superficielle par les eaux de pluie en des paysages hérissés de pointes et d'arêtes vives appelées des Lapiaz. La disparition de la végétation forestière à la suite de l'action de l'homme dans les régions de Nabi Younès et du Jaoubat Bourghaal, a accentué ces phénomènes de corrosion transformant ces régions en un pays de roches, inculte et sec.

La corrosion intense de ces calcaires est une conséquence de leur pureté. En effet, aucun enduit d'argile résiduelle n'atténue leur attaque par l'acide carbonique. Aussi sont-ils d'excellents pierres à chaux. On les utilise également pour la construction et l'empierrement des routes. Ces massifs calcaires sont très fissurés et absorbent presque toute l'eau de pluie qui se trouve ainsi entrainée dans les profondeurs.

En effet, on y observe de véritables gouffres; des rivières peuvent s'y perdre. L'entrainement de l'eau dans les parties profondes de ces massifs, les rend arides, sauvages et inhospitaliers. Ils sont inhabités. C'est le cas du versant oriental $d u$ Djebel Alaouite qui est presque inhabité et couvert par une végétation dégradée. Toute l'eau infiltrée est drainée vers des résurgences peu nombreuses et énormes. Les sources apparaissent en des points bas, fonds de vallée, ou bords de plaines, touchant aux massifs calcaires ou au contact d'autres formations moins perméables. De nombreuses sources jaillissent aux pieds du versant Est du Djebel Alaouite au contact de la plaine du Ghaab, mais malheureusement, ces eaux peuvent être contaminées puisque le calcaire ne les filtre pas et que de nombreuses communications avec la surface peuvent exister.

\subsubsection{Le calcaire dolomitique}

Ce calcaire occupe surtout le versant Ouest du Djebel Alaouite et correspond exclusivement au crétacé moyen. Son épaisseur est considérable, allant de 700 à $800 \mathrm{~m}$. Dans les parties basses du Djebel Alaouite, ce terrain débute par des couches franchement dolomitiques. Plus haut, de gros bancs de calcaires francs alternent avec des marnes dolomitiques à rognons de silex. 
La dolomie mise à nue s'altère par les eaux de pluie, mais conserve à l'altération une forme massive, ce qui permet de la différencier du calcaire compact. Ainsi, dans la série dolomitique des Alaouites, le lapiaz n'apparaitra que dans la partie hante, au niveau des bancs de calcaire tranc.

Ces calcaires sont très fissurés et l'eau $\mathbf{y}$ chemine à peu près comme dans le calcaire compact et se rassemble encore en veines puissantes dont certaines jaillissent au contact avec les plaines ou avec d'autres formations géologiques moins perméables. De petites sources de quelques litres ou quelques dizaines de litres par seconde restent accrochées aux marnes dolomitiques qui alternent avec les bancs calcaires dans la partie haute et attirent les villages.

En résumé, nous pouvons dire que ces calcaires dolomitiques sont plus pénétrables par les eaux de pluie et les eaux y sont mieux réparties. La forêt y pousse mieux que sur le calcaire compact, à cause de leurs réserves hydriques plus élevées.

\subsubsection{La marne crayeuse}

La marne crayeuse est une roche-mère tendre du miocène et occupant dans le N.W. de la Syrie les dépressions et les plateaux. La vallée du Nahr el Kebir et la région Nord de la ville de Lattaquié sont presque entièrement constituées de cette roche.

Cette marne est craquelée, fissurée; les eaux de pluie la pénètrent donc sur une certaine profondeur et la transforment ainsi en une roche altérée, perméable. L'altération porte sur une couche épousant les mouvements topographiques, mince aux saillies, plus épaisse dans les creux. Grâce à cette couche altérée, l'eau de pluie pénètre plus facilement et les filets d'eau cheminent selon la pente, se rassemblent dans les vallées et les plaines en constituant dans leur sous-sol de véritables nappes.

Il est à noter que la végétation forestière favorise l'altération de la marne par suite de l'action des racines et de la matière organique et tend ainsi à faciliter la pénétration de l'eau. De plus, comme cette roche est tendre, elle offre une prise totale au travail de sculpture par les eaux de pluies sauvages et la couche supérieure altérée se trouve ainsi décapée d'une façon continue. La végétation forestière maintient le couche altérée et régularise ainsi 
la pénétration de l'eau de pluie et l'alimentation des nappes, d'où la nécessité de maintenir en permanence sur ces marnes une couverture forestière.

De petites sources ou suintements sont sonvent liés aux ruptures de pente du terrain marneux. Il est aisé d'y aménager des citernes. Ainsi les villages se pressent-ils sur ce terrain facile qui est le plus souvent cultivé. C'est ainsi que toute la région marneuse entre Lattaquié et Wadi Kandil a été déboisée depuis des millénaires et cultivée entièrement.

Dans la partie altérée de la marne, des remontées d'eau par capillarité se produisent en été et humidifient ainsi les couches superficielles. En hiver, la marne devient très boueuse et impraticable. Dans la marne du Nahr el Kabir, on trouve souvent des bancs calcaires poreux et rugueux et quelquefois des conglomérats, des grès ou des sables.

\subsection{LES Roches-MÈRes Basaltiques}

Les terrains volcaniques ne sont pas très fréquents dans le N.W. de la Syrie et s'y trouvent presque exclusivement localisés dans la région littorale entre Banias et Tartous où on aperçoit une plage de couleur noire provenant de la désagrégation du basalte.

Ces zones basaltiques sont très pierreuses et à leur sommet, près de la côte, se dresse Qalaat al Markab.

La végétation forestière a complètement disparu sur ces terrains. Les sols qui s'y sont développés ont été complètement enlevés par érosion à la suite de la suppression de la végétation par l'homme il y a déjà quelques milliers d'années.

Ces roches basaltiques sont le produit d'une activité volcanique récente de la fin du Tertiaire et du Quaternaire. On les appelle basaltes des plateaux. Il se dégagent en croupes rocheuses, de forme en général douce, entourées d'étendues terreuses parsemées de blocs.

\subsection{LES SOLS FOSSILES JOUANT LE RÔLE DE ROCHES-MLiRES}

\subsubsection{La Terra rossa}

La terra rossa est une terre argilense remplissant les fissures et les excavations des calcaires compacts dans le Baer-Bassit 
et le Djebel Alaouite. Elle est surtout répandue dans le Djebel Alaouite et en particulier sur son versant oriental.

La Terra rossa comprend, en mélange, une argile de décarbonatation provenant de la roche calcaire sous-jacente et un complexe détritique constitué d'éléments quartzeux grossiers (MARCELIN, 1947). L'ensemble a subi le phénomène de rubéfaction.

Cette composition prouve que la Terra rossa est une formation géologique ancienne, qui a été formée très probablement sous forêt et sous un climat semblable au climat actuel, mais légèrement plus humide et plus chaud (Durand, 1956). La Terra rossa joue actuellement le rôle de roche-mère et évolue en surface vers des sols actuels entièrement différents en fonction du climat et de la végétation actuel comme on le verra plus loin.

La Terra rossa est une roche compacte, sans structure ou à structure à tendance nuciforme et où l'eau de pluie s'infiltre mal. Sous le climat méditerranéen à saison sèche très accusée du Djebel Alaouite, elle devient très sèche et durcit en surface quand elle n'est pas couverte par une végétation dense.

Sous la forêt climacique, le Quercetum calliprini, ces défauts sont corrigés par l'humus et la Terra rossa évolue vers un sol brun méditerranéen.

De ce qui précède nous dégageons que les districts du Baer et du Bassit sont des massifs siliceux caractérisés par la présence de roches vertes alors que le Djebel Alaouite est un massif entièrement calcaire.

\section{III - LES SOLS}

1. Les sols formés sur péridotites pyroxéniques et serpentines

\subsection{PROPRIÉTÉs ET TYPES}

La décomposition des péridotites pyroxéniques et serpentines dans le Baer-Bassit donne naissance, sous végétation forestière, à des sols du type "brun méditerranéen " à basse altitude, lesquels 
passent à des sols plus ou moins lessivés avec l'augmentation de la pluviosité en altitude.

Examinons le profil $\left(\mathrm{BN}_{2}\right)$ suivant et essayons d'en tirer les principales caractéristiques de ces sols :

Région : Baer

Altitude : $400 \mathrm{~m}$

Exposition : Est

Roche-mère: péridotite pyroxénique serpentinisée

Pluviosité annuelle : 850-900 mm

Pente : $15 \%$

Végétation : étage supérieur: Pinus brutia sous-étage : Phillyrea media, Pistacia palaestina, Styrax offieinale, Cistus villosus, Cistus salviaefolius, ete.

Deseription du profil $\mathrm{BN}_{\mathrm{2}}$

$A_{0} \quad: 2$ à $3 \mathrm{~cm}$ d'épaisseur; formé d'aiguilles de pins et de feuilles non encore décomposées.

I-0.15 $\mathrm{cm}$ : horizon argileux, brun-noir, renfermant des petits blocs de serpentine; structure en gros grumeaux.

II-15-35 : horizon très argileux, compact, rougeâtre, renfermant de petits blocs de serpentine.

III-35-60 : horizon argileux, rouge, compact, renfermant de blocs de serpentine.

IV-60-100 : roche mère en voie de désagrégation.

V-100 et plus : péridotite pyroxénique serpentinisée légèrement altérée.

Le tableau à la page 511 montre l'analyse détaillée de ce profil. De ce tableau nous pouvons déduire les principales propriétés et caractéristiques de ce sol :

1 - Sol jeune, en raison de la présence des blocs de serpentine dans tout le profil.

2 - Sol neutre ou légèrement alcalin et argileux.

3 - Complexe absorbant saturé en bases et surtout en magnésium.

4 - Humus à décomposition rapide du type Mull.

5 - Humification et minéralisation rapides en raison de la grande richesse en bases du sol et de la roche-mère.

6 - Sol riche en oxydes de fer à cause de la roche-mère qui est elle-même riche en fer. La couleur rouge de l'horizon de surface est masquée par la matière organique qui est intimement liée à la matière minérale.

7 - Pas de lessivage du fer.

8 - Tendance nette à l'argilification préférentielle en profondeur. Les sables grossiers (S.G.) et sables fins (S.F.) se transforment très vite en argile en profondeur. En effet, l'argile passe de 36,1\% dans l'horizon III à 55,1 dans l'horizon II et les sables grossiers et fins passent de 16,75 (S.F.) $+30,6$ (S.G.) dans l'horizon III a 11,6 (S.F.) +15,5 (S.G.) dans l'horizon II. Cette formation préférentielle de l'argile en profondeur est très utile à la végétation forestière de notre région. L'horizon argileux en profon- 
deur constitue une sorte de réserve d'eau à l'abri de l'évaporation que les arbres peuvent utiliser pendant la période sèche estivale.

9 - Ce sol est un sol brun méditerranéen jeune.

Reifenberg et Eubank (1933) ont étudié les sols formés sur serpentine à Chypre dans des conditions voisines des nôtres. Ils ont trouvé les mêmes résultats et appelé ces sols " reddish-brown mediterranean soils".

Ces sols bruns méditerranéens passent à des sols plus ou moins lessivés avec l'augmentation de la pluviosité en altitude comme le montre le profil B suivant :

Région : Bayer

Altitude : $600 \mathrm{~m}$

Roche-mère : pértidotite pyroxénique serpentinisée

Pluviosité annuelle : $950-1000 \mathrm{~mm}$

Pente : $15 \%$

Végétation : étage supérieur : Pinus brutia

sous-étage: Quercus pseudocerris, Cistus villosus, Cistus salviaefolius, Ptosimopappus bracteatus, etc.

Description $d u$ profil $B$

$\mathbf{A}_{0}$

$1.3 .25 \mathrm{~cm}$

II $-25-80 \mathrm{~cm}$

III $-80.100 \mathrm{~cm}$ : horizon très argileux, compact, rouge
IV $120 \mathrm{~cm}$ et plus : péridotite pyroxénique serpentinisée. feuilles mortes
: 2 à $3 \mathrm{~cm}$ d'épaisseur; formé d'aiguilles de pins et de

: horizon argileux, brun-noir, à structure en gros grumeaux : horizon argileux, brundtre, structure grumeleuse : horizon très argileux, compact, rouge

Le tableau à la page 511 montre l'analyse détaillée de ce profil. De ce tableau nous pouvons déduire les propriétés et les caractéristiques de ce sol :

1 - Sol légèrement alcalin $(\mathrm{pH}=\mathbf{7 , 5})$ et argileux.

2 - Complexe absorbant saturé en bases et surtout en magnésium.

3 - Sol complètement décarbonaté.

4 - Pourcentage de la matière organique de l'horizon de surface de l'ordre de 4 à $4,5 \%$.

5 - Humus à décomposition rapide du type Mull.

6 - Lessivage du fer assez marqué, passant de 7,9\% $\left(\mathrm{Fe}_{2} \mathrm{O}_{\mathrm{g}}\right)$ dans l'horizon I a $12,4 \%$ dans l'horizon II.

7 -- Tendance nette à l'argilification préférentielle en profondeur. L'argile passe de $29 \%$ dans l'horizon III à $45,2 \%$ dans l'horizon II et les sables grossiers et fins passent de 48,8\% (S.G.) + 13,3 (S.F.) dans l'horizon III à $24,3 \%$ (S.G.) $+11,3 \%$ (S.F.) dans l'horizon II. Le pourcentage de limon augmente aussi et passe de $8,5 \%$ à $17,7 \%$.

8 - Ce sol est un sol brun méditerranéen lessivé. 
LA végetation DU BaER-Bassit et dU DJ. alaOUtTe (STRIE) 511

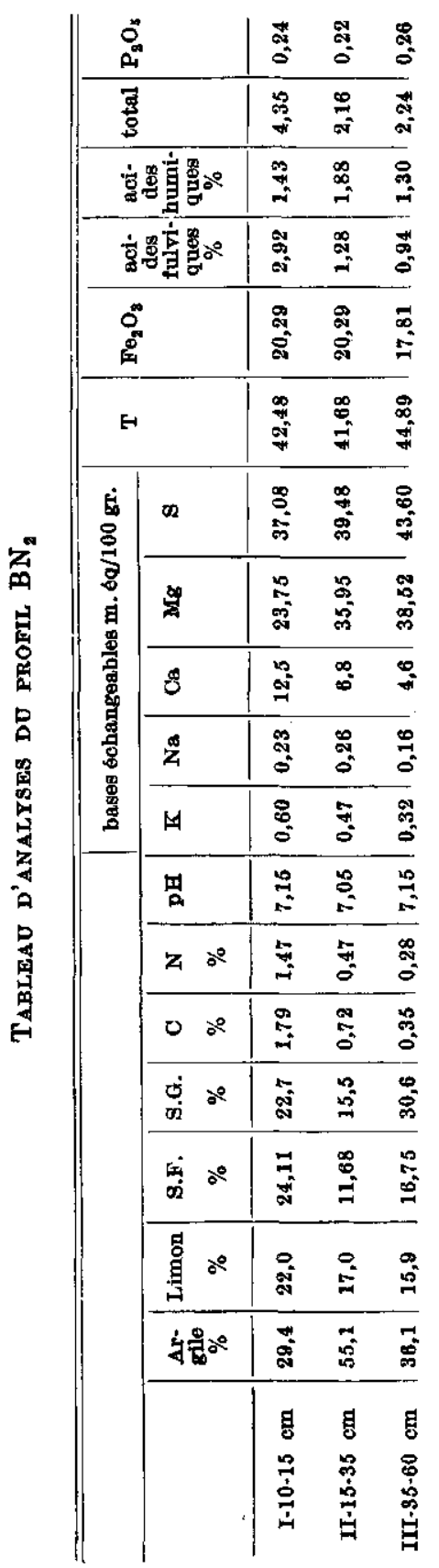

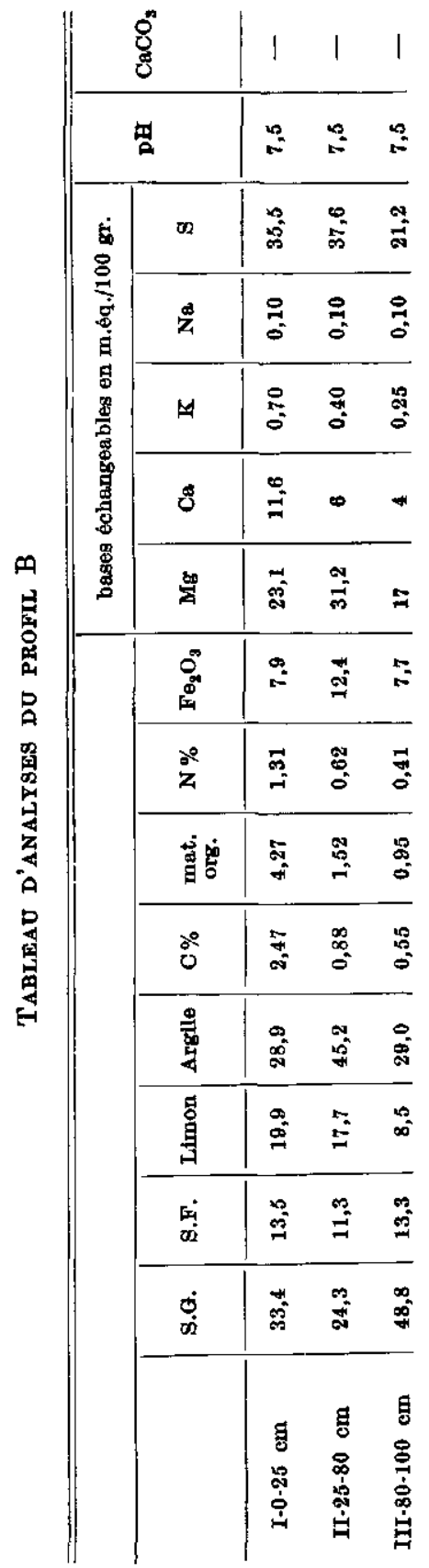




\subsection{VÉGETATION}

Le Pinus brutia pousse bien sur ces sols et y constitue de belles forêts. Il se mélange quelquefois au Quercus pseudocerris sur les expositions fraiches et humides et constitue ainsi des forêts mixtes feuillus-résineux que nous traiterons dans le chapitre quatrième.

\subsection{Dégradation}

Ces sols se dégradent à la suite des coupes abusives non contrôlées, des incendies répétés et du pâturage intensif qui ont pour conséquence de découvrir fréquemment le sol et le soumettre à l'action directe des raysons solaires, du vent et des averses.

L'humus déjà insuffisant, disparaît presque entièrement par minéralisation. Cette minéralisation est très rapide dans le Baer Bassit à cause du climat chaud et de la grande richesse de ces sols en bases et en particulier en magnésium. Comme cet humus consitue le ciment des agrégats, la structure est détruite et le sol se tasse ; l'argile et les oxydes de fer sont dispersés et libérés. Le sol devient ainsi très sensible à l'érosion. Les très fortes pluies, fréquentes dans la région, entraînent la terre fine graduellement.

La première conséquence de la disparition du peuplement forestier est done la disparition de l'humus forestier des horizons supérieurs et l'entraînement à la longue de ces horizons. Le sol s'appauvrit par perte d'humus, de terre fine et de cations. Le cycle des cations et de l'azote qui se produit habituellement sous végétation forestière n'a pas lieu. Les oxydes de fer libérés se déshy. dratent sous l'action des raysons solaires et le sol prend une teinte rouge. La déshydratation de ces oxydes est beaucoup plus intense sur les expositions sud. Sur les expositions Ouest et Nord-Ouest, ce phénomène est moins accusé.

La disparition de l'humus et l'entraînement de l'horizon humifère mettent à nu les borizons rouges profonds. Le sol brun forestier méditerranéen se 1rouve ainsi remplacé par un sol rouge typique. 
Si la dégradation continue, ce sol rouge se trouve lui-même entrainé et la serpentine apparaît en surface dénudée : seule parfois, une très mince couche d'argile et d'humus subsiste en surface : le sol devient un ranker d'érosion et dans le cas extrême un lithosol.

Nous pouvons schématiser la dégradation des sols bruns forestiers méditérranées sur serpentine de la façon suivante:

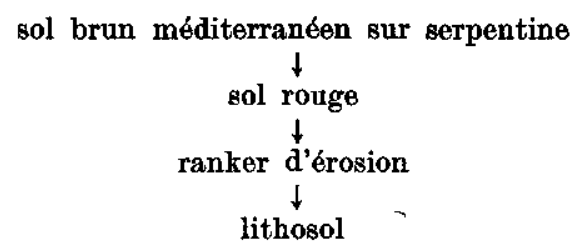

La végétation forestière suit la même évolution régressive et, à la longue, se trouve remplacée par une pelouse à graminées xérophiles sans valeur économique.

En contrôlant bien les coupes, afin de ne pas découvrir le sol, en maintenant un mélange d'essences et un sous-étage, le forestier peut empêcher la dégradation de la forêt et maintenir d'une façon permanente la fertilité des sols.

\section{Les sols formes sur gabbros}

\subsection{Proprítét́s et types}

La décomposition des gabbros donne naissance, dans le Baer et le Bassit, à des sols du type brun méditerranéen. Ces sols sont, en général, plus profonds que ceux formés sur serpentine en raison de la facilité d'altération des gabbros, et plus riches en bases $\left(\mathrm{CaO}, \mathrm{Na}_{2} \mathrm{O}\right)$, mais ils sont moins riches en magnésium et en fer.

L'examen du profil $\mathrm{BN}_{3}$ suivant nous permettra de dégager les principales propriétés et caractéristiques de ces sols (A. REIFEnBERG 1952) :

Région : route Lattaquié-Kassab à $2 \mathrm{~km}$ au sud de la frontière

Altitude : $700 \mathrm{~m}$

Pluviosité : $1000 \mathrm{~mm}$

Végétation : forêt claire de Pinus brutia

Pente : très faible. 
Description du profil

I.0-20 $\mathrm{cm} \quad$ : horizon argileux, grisâtre

II-20-25 cm : horizon jaunatre, argileux, avec des grains de gabbros en voie de décomposition

III-25 cm et plus : horizon gris, en partie décomposé ; présence de gabbro non décomposé et en voie de décomposition avec des plagioclases légèrement colorés, anorthite et du pyroxìne très foncé.

Il n'y a pas d'orizon humifère. Il est très probable que l'horizon humifère fût décapé par érosion.

L'analyse de ce profil a donné les résultats suivants :

1 - Sol neutre ou légèrement alcalin $(\mathrm{pH}=7,2)$ et argileux.

2 - Complexe absorbant saturé en bases.

3 - Lessivage assez important $d u$ fer, du calcium et du magnésium et accumulation dans l'horizon II. La coloration jaunatre de cet borizon est due à cette accumulation de fer.

C'est un sol lessivé.

\subsection{VÉGÉtation}

Ces sols sont occupés le plus souvent par le Quercus pseudocerris qui y constitue de belles forêts denses et en très bon état. Le Quercus pseudocerris est accompagné des espèces suivantes : Ostrya carpinifolia, Carpinus orientalis, Querous infectoria, Styrax officinalis, Digitalis ferruginea, etc.

\subsection{Degradation}

Sous l'influence des coupes abusives et non contrôlées, ces sols se dégradent de la même façon que les sols formés sur serpentines par disparition de l'humus, des horisons humifères et de la terre fine. Mais leur position topographique et la rapidité d'altération de la roche-mère les rend moins sensibles à l'érosion que les sols formés sur serpentines. 
LA VEgttation DU BaER-Bassit ItT DU DJ. aLAOdite (SYRIE) 515

Tablead d'analyse du profil BN,

\begin{tabular}{|c|c|c|c|c|c|}
\hline & III & II & I & & $\%$ \\
\hline $\mathrm{SiO}_{2}$ & 56,55 & 56,99 & 73,96 & S.G. & 1,48 \\
\hline $\mathrm{Al}_{2} \mathrm{O}_{3}$ & 6,65 & 7,71 & 8,17 & S.F. & 29,13 \\
\hline $\mathrm{Fe}_{2} \mathrm{O}_{3}$ & 10,17 & 14,48 & 8,35 & limon & 18,93 \\
\hline $\mathrm{FeO}^{\circ}$ & 0,28 & 0,47 & 0,55 & argile & 43,37 \\
\hline $\mathrm{CaO}$ & 10,18 & 8,47 & 3,12 & calcaire & 0,68 \\
\hline $\mathrm{MgO}$ & 15,89 & 10,17 & 4,05 & $\mathrm{H}_{2} \mathrm{O}$ & 6,14 \\
\hline $\mathrm{K}_{\mathbf{2}} \mathrm{O}$ & tr & 0,20 & 0,07 & & \\
\hline $\mathrm{Na}_{2} \mathrm{O}$ & tr & 1,42 & 0,60 & & \\
\hline$P_{2} 0_{5}$ & 0,31 & 0,37 & 0,47 & & \\
\hline $\mathbf{S i} / \mathbf{A l}$ & 14,5 & $12, \theta$ & 15,4 & & \\
\hline $\mathrm{Si} / \mathbf{F e}$ & 14,3 & 10,1 & 21,9 & & \\
\hline $\mathrm{Si} / \mathrm{R}$ & 7,2 & 5,6 & 9,1 & & \\
\hline $\mathrm{pH}$ & & & 7,2 & & \\
\hline
\end{tabular}

\section{Les sols formés sur caleaire compact et Terra rossa}

\subsection{Propriteths et TXPES DE Sols}

Le calcaire compact est recouvert par une terre argileuse, la Terra rossa, qui remplit ses fissures et excavations. Cette Terra rossa, comme on l'a déjà dit, est un sol fossile, qui joue le rôle d'une véritable roche mère et qui subit une évolution pédologique récente en liaison avec le climat et la végétation actuels.

\subsubsection{Sols bruns méditerranéens sur Terra rossa}

Sous végétation forestière, la Terra rossa donne naissance ̀̀ un sol brun par accumulation d'humus et formation d'horizon humifère brun en surface. Cet horizon humifère est d'autant plus épais que la végétation est plus dense et d'installation plus ancienne. Sous la forêt climacique de Quercus calliprinos, le Quercetum calliprini, entre 300 et $800 \mathrm{~m}$ d'altitude dans le Djebel Alaouite, la Terra rossa donne naissance à un sol brun méditerranéen (ou sol rouge brunifié). Le traitement en taillis des forêts à Quercus calliprinos a pour effet, l'amincissement des horizons humifères et la diminution de la quantité d'humus accumulée dans le profil, à la suite des coupes qui découvrent très fréquemment le sol. 


\subsubsection{Rendzines rouges}

Sur les pentes et sous forêt, la Terra rossa évolue vers une rendzine rouge, par remaniement de la terre rouge et de fragments de roches calcaires. La rendzine rouge est donc un sol de formation secondaire. Ces rendzines rouges diffèrent des sols précédents par les caractères suivants :

- Présence de nombreux cailloux calcaires parsemés dans le profil et mélangés à la Terra rossa;

- structure grenue;

- présence de nombreux vers de terre qui dénotent une grande activité biologique;

- teneur élevée en calcaire actif; le pH est de 1'ordre de 8 ;

- teneur plus élevée en humus bien réparti dans le profil.

\subsubsection{Sols humiques carbonatés}

En hautes altitudes, et sur les pentes, la Terra rossa, donne naissance sous végétation forestière, à des sols humiques carbonatés (souvent appelés rendzines noires d'altitude) caractérisés par un mélange peu intime d'humus et de fragments de calcaire compact. Le froid qui sévit en haute altitude ralentit beaucoup l'évolution de l'humus qui s'accumule ainsi dans le profil. Ces sols sont fréquents sur les "lapiaz" du Djebel Alaouite.

\subsection{VÉGÉTATION}

Le sol brun méditerranéen représente le sol climacique entre 300 et $800 \mathrm{~m}$ d'altitude, correspondant à l'association climacique le Quercetum calliprini.

Les sols bruns méditerranéens jeunes et les rendzines ronges sont occupés le plus souvent par une végétation secondaire de garrigue dे Quercus calliprinos et Pistacia palaestina (PistacietoQuercetum calliprini). Les sols humiques carbonatés, qui se trouvent en haute altitude sont occupés par le Cedretum libani et. l'Abietum cilicicae. 


\subsection{Degradation des sols bruns méditgranáfens sur Terra ROSSA}

Nous avons dit plus haut que le sol brun méditerranéen sur Terra rossa est un sol-climax (sol analogue) caractéristique de la forêt climax de Quercus calliprinos (Quercetum calliprini). La dégradation de la forêt climacique par les coupes abusives et non contrôlées et le pâturage provoque, en même temp, la dégradation du sol climacique. La végétation primitive donne naissance à une garrigue à Quercus calliprinos et Pistacia palaestina qui se dégrade à son tour par suite de la continuité de l'action de l'homme et du pâturage et donne naissance à des associations de plus en plus dégradées couvrant très mal le sol et donnant moins de matière organique (voir chapitre cinquième). Le sol climax suit la même évolution; l'humus disparaît graduellement, ainsi que les horizons bruns humifères de la surface et la Terra rossa se trouve ainsi mise a nu.

Si la dégradation continue, la terre rouge se trouve entraînée par érosion, et le calcaire compact apparaît en surface, le sol devient un lithosol.

Ce processus d'évolution régressive du sol brun méditerranéen sur Terra rossa peut être élucidée par le profil suivant de la région de Safita, dont l'évolution est étroitement liée au régime du traitement en taillis du Quereus calliprinos :

Région : près de Safita

Altitude : $230 \mathrm{~m}$

Pluviométrie : $850 \mathrm{~mm}$

Végétation : garrigue à Quercus calliprinos et Pistacia palaestina.

\section{Description du profil}

$A_{0}-2 \mathrm{~cm} \quad:$ formé de litière non décomposée

$A_{1} \cdot 0.15 \mathrm{~cm} \quad$ : horizon noirâtre, humifère, renfermant $2,5 \%$ d'humus, présence d'abondantes racines, $\mathrm{pH}$ voisin de 7,5

B-C $-\mathbf{1 5 - 3 0} \mathrm{cm} \quad$ : horizon argileux rouge, dépourvu de carbonate, à structure granulaire; racines fréquentes; cet horizon pénètre dans les fissures du calcaire compact sous-jacent.

D - 30 cm et plus : calcaire cristallin. 
Ce sol est décarbonaté, mais il est saturé on calcium : voici les bases échangeables pour l'horizon $\mathbf{A}_{\mathbf{1}}$ :

\begin{tabular}{c|c|c|c|c}
\hline \hline \multicolumn{2}{c}{ basea 6changeables on m. 6q. Dorr $100 \mathrm{gr}$. de terre } \\
\hline $\mathrm{Ca}$ & $\mathrm{Mg}$ & $\mathrm{K}$ & $\mathrm{Na}$ & $\mathrm{s}$ \\
\hline 57,5 & 1,00 & 1,30 & 4,20 & 64 \\
\hline
\end{tabular}

Avec son horizon humifère réduit, ce sol est un. sol rouge méditerranéen typique.

L'évolution progressive et régressive des sols formés sur Terra rossa peuvent être schématisées de la façon suivante:

sol brun mediterranéen (climax)

(forêt à Quercus calliprinos - climax)

sol rouge méditerranéen (garrigue à Quercus calliprinos

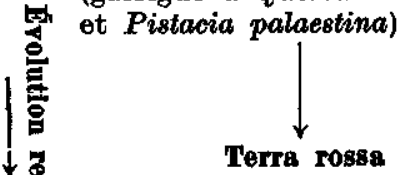

(lande a Poterium spinosum et Genista acanthoclada; pelouse sèche à Asphodelus microcarpus)<smiles>[14CH3]</smiles>

(calcaire compact) sol brun méditerranén jeune (lande à Calycotome villosa ; garrigue a Quercus calliprinos ot Pistacia palaestina)

Terra rossa sur calcaire compact (plantes pionnières)

\section{Les sols formés sur marne crayeuse}

\subsection{Propritetes ets types}

Nous avons vu que la marne crayeuse du Nord-Ouest de la Syrie est une roche-mère tendre, meuble et assez riche en argile. Sous végétation forestière, elle donne naissance à des sols bruns calcaires, sols de transition vers les sols bruns. 
Les sols bruns calcaires sont caractérisés par une décarbonatation incomplète, une structure polyédrique en raison de la richesse de la roche-mère en argile. On n'y observe pas de cailloux. Le pH est voisin de 8.

La présence de bancs calcaires dans la marne fait évoluer celle-ci vers une rendzine qui diffère du sol brun calcaire par la présence de cailloux calcaires dans le profil et une structure grenue. Le $\mathrm{pH}$ des rendzines est toujours élevé ; il atteint et même dépasse 8. Ces rendzines sont assez riches en humus de néoformation microbienne, réparti de façon relativement homogène dans le profil. Cet humus est un "Mull calcique " qui donne une structure stable. Ces rendzines s'observent surtout sur les pentes à cause du brassage constant des horizons causés par l'érosion et l'activité animale.

Chez les rendzines, l'érosion provoque une accumulation de cailloux en surface qui forment écran à l'égard de l'évaporation. De plus, dans la marne, des réserves hydriques peuvent se constitner en profondeur. La régénération du Pinus brutia se fait plus aisément sur ces sols que sur Terra rossa ; ses semis y résistent mieux à la sécheresse.

\subsection{Végétation}

Les sols bruns calcaires et les rendzines sont occupées par la garrigue à Quercus calliprinos et Pistacia palaestina, 1'association à Ceratonia siliqua et Pistacia Lentiseus et les forêts secondaires de Pinus brutia. Beaucoup de ces sols sont actuellement occupés par l'agriculture et l'arboriculture et surtout par l'olivier et les figuiers à la suite des déboisements qui ont eu lieu depuis une époque très lointaine.

\subsection{DÉgRAdation DES SOLS BRUNS CALCAIRES ET DES RENDZiNES}

Le sol brun calcaire se dégrade sous l'influence des coupes abusives, trop répétées qui ont pour effet de découvrir le sol, et du pâturage intensif. L'humus disparaît par minéralisation et 
comme il constitue le ciment des agrégats, la structure se détruit, le sol se tasse et devient ainsi très sensible à l'érosion qui entraine les horizons meubles supérieurs. Le sol se trouve ainsi dépourva de ses horizons humifères et réduit aux couches marneuses des horizons inférieurs peu perméables à l'eau, et devient un sol brut sur marne.

La végétation forestière primitive suit la même dégradation et se trouve remplacée par des pelouses sèches à graminées.

Les rendzines se dégradent de la même façon par disparition graduelle des horizons humifères. Dans le cas extrême, la rendzine est réduite à la roche calcaire (lithosol).

Ces sols dégradés sont malheureusement assez répandus dans le Djebel Alaouite.

\section{Les sols formés sur calcaire dolomitique}

Les sols formés sur calcaire dolomitique ne diffèrent pas beaucoup des sols formés sur calcaire marneux. Ils donnent naissance à des rendzines que nous appellerons rendzines magnésiennes pour les différencier des rendzines formées sur calcaire marneux.

Ces rendzines magnésiennes sont très voisines des vraies rendzines et n'en diffèrent que par leur richesse en magnésie et leur structure moins stable. En outre, la végétation qui y pousse est la même que celle qui pousse sur les rendzines vraies.

(1) (A) : Horizon différent de la roche-mère par une simple désagrégation physique.

(') (B) : "B structural ", différent d'une part de la roche-mère, par son degré d'altération plus fort (présence de $\mathrm{Fe}_{2} \mathrm{O}_{3}$ libre); d'autre part de l'horizon de surface A par sa structure différente, en général plus compacte et par l'absence de matière organique.

$\left(^{2}\right) \mathrm{g}$ : pseudogley, se formant au sein d'une nappe d'eau temporaire; tâches grises, appaurries en fer, sur fond ocre.

(4) G : Gley de couleur gris verdatre, a tâche rouille, se formant au sein ou à la limite supérieure d'une nappe phréatique permanente. 
TABLEAU DE Classification Des SOLS FOREstiers

dU Baer-Bassit et dU DJebel Alaotrte

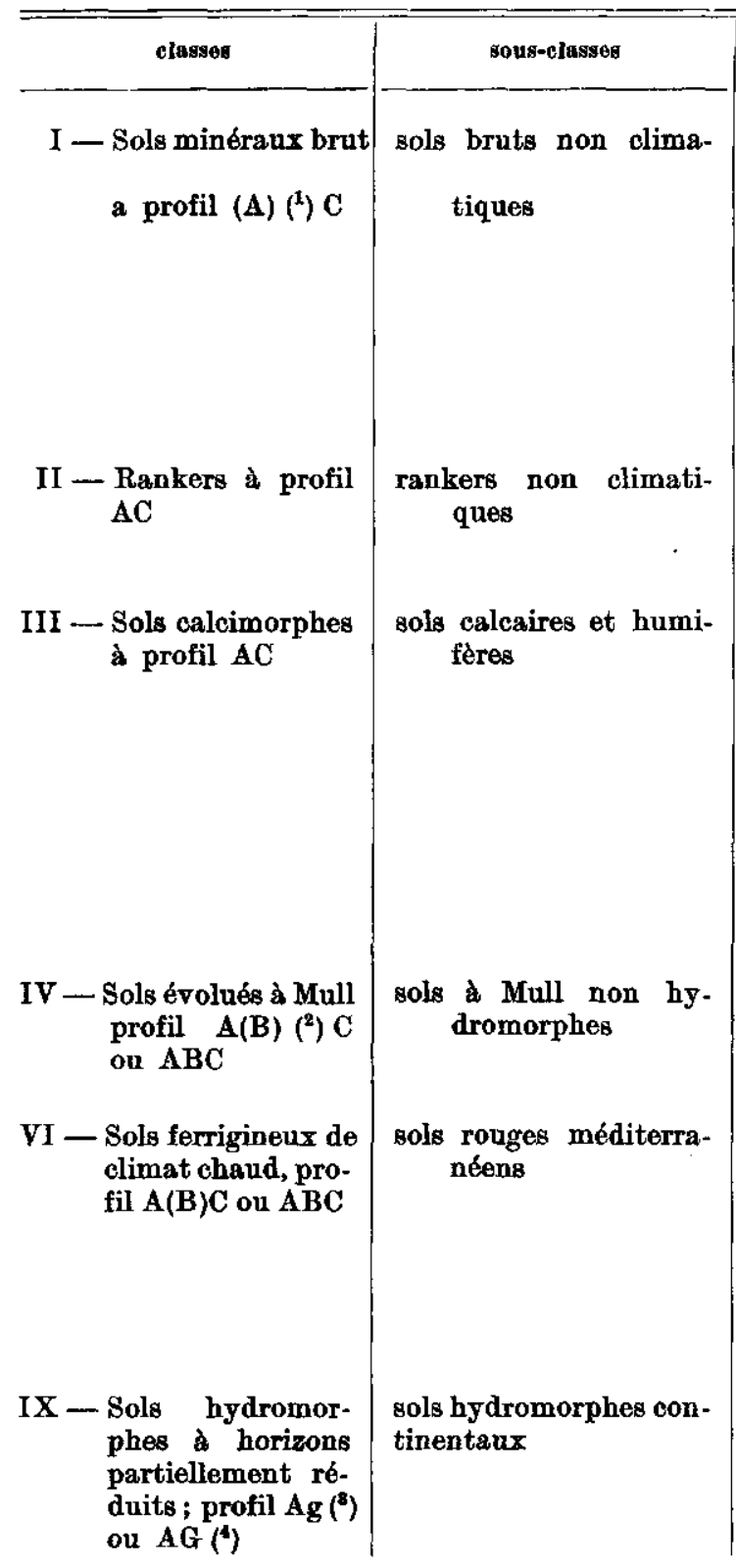

groupes
sols bruts d'érosion calcaire compact.
- lithosols : provenant de la dégradation des sols bruns méditerranéens sur serpentine, gab- bros, calcaire compact.
-régosols : sol brut de marne

rankers d'érosion sur serpentine, gabbros, basalte

a. rendzines vraies sur calcaire marneux

b. rendzines rouges provenant d'un remaniement de Ia Terra rossa

c. rendzines magnésien. nes sur calcaire dolomitique

d. bruns calcaires sur marnes

sols bruns lessivés ou lessivés dans les régions à grande pluviosité

a. sol rouge méditerranéen non léssivé : provenant de la dégradation des sols bruns méditerranéens

c. sol rouge brunifié (sol brun méditerranéen)

a. pseudogley de surface

b. pseudogley de profondeur

c. gley

(Les notes à la page 520 ) 
6. La systématique des sols forestiers du Baer-Bassit et du Djebel Alaonite

Nous avons utilisé la classification internationale proposée par G. Aubert et Ph. Duchaufour au Congrès International de la Science du sol à Paris, en 1956. Dans cette classification, les sols sont groupés en 10 classes principales, d'après les caractères fondamentaux de l'évolution, notamment (DUCHAUfOUR 1960) :

1) le degré de l'évolution conduisant à une différenciation du profil de plus en plus marquée;

2) la nature physico-chimique de l'évolution liée, elle-même, à trois propriétés essentielles : les conditions de l'altération, le type d'humus et le chimisme du complexe absorbant.

Les sous-classes se séparent, plus souvent, par le facteur écologique de base, qui conditionne l'évolution (climat, roche-mère, conditions de stations influençant le régime hydrique).

Les groupes diffèrent entre eux par une particularité de processus évolutif, par exemple, intensité de l'altération ou degré de lessivage des colloïdes.

Cette classification a l'avantage d'être logique, générale et en même temps applicable. Elle convient bien à la classification des sols forestiers que nous avons étudiés dans le Baer-Bassit et le Djebel Alaouite.

Nous reproduisons dans le tableau suivant les classes, sousclasses et groupes des sols qui existent dans notre région et que nous avons étudiés dans ce chapitre. Nous avons conservé le numéro donné par les auteurs à chaque classe, dans la classification internationale. Les classes des sols qui n'existent pas dans notre région n'ont pas été représentées dans le tableau.

Notre étude des sols forestiers du Baer Bassit et du Djebel Alaouite constitue une première contribution à la connaissance des sols de ces deux régions. Elle est, de ce fait, incomplète. Mais les grandes lignes de la pédogénèse et les principaux groupes $y$ sont déjà signalés.

Cette première tentative constitue un cadre destiné à être élargi, précisé et complété par des études ultérieures plus détaillées qui permettront de préciser les sons-groupes ou les types génétiques. 


\section{IV - INFL UENCE DES VERSANTS}

Le contraste des versants se fait sentir d'une façon très nette dans le Baer Bassit et le Djebel Alaouite. Pour comprendre l'effet des versants sur la végétation dans notre région, il est utile de signaler que les vents humides qui apportent les pluies sont des vents d'Ouest ou Sud-Ouest. Les versants exposés à l'Ouest et au Sud-Onest sont done des versants humides, alors que les versants exposés à l'Est sont des versants secs.

Le Djebel Alaouite, nous l'avons déjà vu, a une orientation Nord-Sud, parallèle à la côte. Il arrête les vents pluvieux qui abandonnent leur humidité sous forme de pluies brutales sur son versant Ouest et sur le littoral.

Le littoral et les versants Ouest du Djebel Alaouite et du Baer-Bassit sont des régions les plus arrosées de Syrie. En outre, ces chaînes de montagnes côtières constituent une barrière climatique qui exerce une action profonde sur le climat des régions intérieures de Syrie, dont la sécheresse augmente an fur et à mesure que l'on s'éloigne de la côte.

Les versants exposés au Nord sont ombragés, sans soleil ou éclairés seulement par des rayons obliques ou frisants; ils sont frais et subissent le minumum d'évaporation; l'humus s'y maintient aisément et le sol y demeure frais.

Par contre, sur les versants exposés au Sud, le soleil produit tous ses effets. Quelles que soient les pentes, il y a toujours des heures et des saisons ou ses rayons frappent les versants perpendiculairement à leur surface largement éclairée ; elles subissent le maximum d'évaporation; l'ean de pluie qui ne ruisselle pas est bien vite enlevée au sol qui en emmagasine peu ; le sol s'échauffe beaucoup.

Chaque versant (Nord, Sud, Est, Ouest) se caractérise donc par des conditions écologiques spéciales qui sont réflétées d'une façon très nette par la végétation.

Les versants humides et les versants frais (Ouest et Nord), ont une végétation luxuriante; les forêts y poussent avec vigueur. En outre, cette végétation est composée d'essences forestières exigentes en humidité. C'est ainsi que les versants Ouest et Nord 
ouest du Baer Bassit sont occupés à partir de $450 \mathrm{~m}$ d'altitude, par une espèce hydrophile, à feuilles très larges et caduques, le Quercus pseudocerris et la partie supérieure du Djebel Alaouite est occupée par l'Abies cilicica, essence forestière aimant le climat froid et humide.

Malgré les abus d'exploitations de l'homme depuis des millénaires, ces versants sont mieux conservés que les versants Sud et Est. Dans une région comme le Baer Bassit et le Djebel Alaouite, où l'été est très sec, le facteur eau joue un rôle très important dans la répartition et le développement de la végétation forestière. L'influence de la sécheresse se fait sentir beaucoup plus sur les sols calcaires sees que sur les sols siliceux. C'est ainsi que le Quercus pseudocerris apparaît à partir de $450 \mathrm{~m}$, dans le Baer Bassit et sur substratum siliceux alors que le Quercus Cerris, qui a une écologie analogue, n'apparâit qu'à partir de $800 \mathrm{~m}$ dans le Djebel Alaouite où le substratum est calcaire.

En outre, les essences forestières qui dominent sur les versants frais et humides ne sont pas celles qui prospèrent aux mêmes niveaux sur les versants secs et chauds.

Les versands Sud et Est sont moins favorables que les versants Ouest et Nord à la végétation forestière à cause de leur sécheresse accrue. La végétation y a une écologie plus xérophile. La partie supérieure du versant Est du Djebel Alaouite est occupée par le Cedretum libani, association plus xérophile que l'Abietum cilicicae qui occupe à la même altitude le versant Nord.

Le complexe sol-végétation est aussi très fragile sur ces versants. Les coupes abusives et non contrôlées et les pâturages y provoquent des désastres. Le sol se dégrade très vite, l'humus disparaît et à la longue la terre fine finit par disparaître à son tour par érosion. Le sol devient très sec et défavorable à la régénération de la forêt primitive qui se trouve ainsi remplacée par une végétation secondaire plus xérophile.

Le forestier doit être très prudent sur les versants Est et Sud. Ses coupes doivent être faites de façon à découvrir le moins longtemps possible le sol. Le pâturage doit y être très bien réglementé. 


\section{V - L'HOMME EN TANT QUE FACTEUR DU MILIEU}

\section{Groupements elimaciques - discussion}

Un groupement végétal climacique (ou phytoclimax) est un groupement dont les individus sont floristiquement stables, représentant un état d'équilibre floristico-écologique, qui est fonction des conditions générales - flore, climat, faune, substratum géologique - mais dans la réalisation et le maintien duquel la végétation joue un rôle décisif (GuINochET 1955). Le sol correspondant est un sol climacique ou pédoclimax.

Lorsqu'un "individu " d'un groupement climacique est victime d'une rupture de l'équilibrie, souvent fragile, auquel il doit son existence, il se déclenche habituellement une série régressive de combinaisons floristiques se succédant plus ou moins rapidement et pouvant, suivant la nature, l'importance et la durée de la ou des causes perturbatrices, soit s'arrêter à un stade donné, soit conduire, dans les cas extrêmes, jusqu'à la nudité du substratum minéralogique. A partir de là, si des conditions favorables sont de nouveaux réalisées, une nouvelle série progressive peut s'amorcer, sans d'ailleurs comporter obligatoirement la même succession des groupements et aboutir au même climax. Les groupements végétaux réseultant de la rupture d'équilibre du groupement climacique sont appelés les groupements transitoires.

La différence entre groupements climaciques et groupements transitoires tient essentiellement dans le fait que les "individus" des premiers sont floristiquement stables dans le temps, anssi bien du point de vue qualitatif que quantitatif. Les fluctuations aléatoires y sont de faible amplitude et correspondent à des écarts périodiques ayant des effets sensiblement égaux de part et d'autre du type moyen.

Chez les groupements transitoires, au contraire, les variations floristiques qualitatives et quantitatives des « individus 》 d'associations sont polarisées : an cours du temps, certaines espèces diminuent en quantité, pour finir par disparaître, tandis que d'autres apparaissent et voient leur abondance augmenter plus ou moins. Dans ces groupements transitoires, on trouve à côté d'un noyau 
d'espèces propres au groupement, des espèces reliques du groupement précédent et des espèces pionnières du suivant.

Un groupement permanent est une combinaison floristique qui s'installe et persiste dans un milieu physique si spécial (pente d'éboulis très inclinée, station très salée) qu'elle est incapable de modifier et qui n'est, de ce fait, pas remplacée par d'autres au cours du temps (GurNochet 1955).

Le groupement permanent offre la même stabilité floristique dans le temps que le groupement climacique. La différence tient alors dans le fait que, chez le premier, la végétation est contrainte de se contenter, en qualque sorte, des conditions qui lui sont offertes sans pouvoir leur imprimer profondément sa marque. Tandis qu'un groupement climacique représente un état d'équilibre végétation-sol, en fonction du climat général et de la faune du territoire, état d'équilibre qui est l'aboutissement d'une évolution parallèle dans laquelle la végétation joue un rôle décisif.

Le cas idéal est celui où les conditions générales sont telles qu'il y a réalisation du même ensemble phytoclimax-pédoclimax à partir de roches-mères distinctes. Ce cas est assez rare dans la nature et ne se trouve réalisé que dans les régions où le climat empêche la roche-mère de manifester son influence sur le sol. Or, dans les pays méditerranéens, et en particulier dans la région que nous étudions, où règne un climat méditerranéen à saison très sèche, la roche-mère manifeste fortement son influence sur le sol et par voie de conséquence, sur la combinaison floristique correspondante. Sous un même climat général, mais sur des roches-mères variées, nous aurons donc dans notre région (comme on le verra plus loin) des groupements climaciques variés.

Il résulte de ce qui précède, que la notion de climax est assez subtile et qu'en fait il $\mathrm{y}$ a tous les intermédiaires entre le climax idéal, se réalisant identique à lui même sur des substratums géologiques différents et les groupements permanents.

2. Le rôle de l'homme dans l'evolution des groupements végétaux dans le Baer-Bassit et le Djebel Alaouite

Nous avons vu plus haut que l'évolution de la végétation ne consiste pas seulement en une simple confrontation Flore-Milieu 
physique. Le substratum géologique et la faune jouent également un rôle. Parmi les éléments de la faune, il en est un dont l'action sur la végétation a été très importante dans la région que nous étudions: e'est l'homme.

L'homme a habité le Nord-Onest de la Syrie et la côte de la Méditerranée orientale depuis des millénaires. Les recherches faites sur le Quaternaire côtier de Tripoli au Liban (voir chapitre 3) ont montré que les premières manifestations humaines observées précèdent immédiatement la première époque pluviale.

L'homme a commencé à défricher le littoral et s'y est installé, laissant les montagnes couvertes de forêts sombres, inhabitées. Ces forêts étaient considérées pendant toute l'Antiquité comme des réserves disputées de bois. Les documents égyptiens, assyrobabyloniens, la Bible, les textes grecs et latins, les textes et documents du Haut Moyen âge eux-mêmes attestent l'importance des boisements au Liban, Djebel Alaouite et Amanus, et une exploitation méthodique de la forêt pour les besoins de constructions terrestres et navales (v. ch. 4: Cedrus). L'occupation hamaine des massifs du Moyen-âge s'est effectuée par défrichements et s'est prolongée jusqu'à l'époque contemporaine. Les dernières exploitations massives et désastreuses de nos forêts ont été effectuées par les Tures pendant la guerre de 1914-1918.

L'homme a, par conséquent, bouleversé l'équilibre de la nature dans cette région, soit en défrichant des régions boisées pour les mettre en culture, soit en exploitant les forêts pour satisfaire ses besoins multiples ou pour un but commercial.

Les exploitations continuelles et abusives des forêts qui représentent les groupements végétaux naturels de notre région, ont eu pour résultat la disparition presque totale de certains groupements climaciques et leur remplacement par des groupements transitoires variés suivant la nature de la roche-mère.

Un exemple typique de l'évolution des groupements végétaux sous l'action de l'homme, dans notre région est celui de la forêt climacique de Quercus calliprinos du Djebel Alaouite: le Quercetum calliprini Alaouitaea (voir chapitre cinquième). Cette association qui couvrait autrefois la zone comprise entre 200-300 et $800 \mathrm{~m}$ d'altitude dans le Djebel Alaouite n'existe plus de nos jours que sous forme de petits lambeaux de quelques centaines 
de mètres carrés, autour des lieux sains (Mazars). Elle a été remplacée par une garrigue à Quercus calliprinos et Pistacia palaestina (Pistacieto-Quercetum calliprini Alaouitaea) laquelle a été remplacée à son tour, en beaucoup de points, par des associations plus dégradées de landes et de pelouses sèches. Dans le cas extrême de la dégradation, la roche-mère apparaissait en surface.

Quand l'action de l'homme cessait, ou du moins diminuait, une évolution progressive se déclenchait. Des groupements transitoires nouveaux prenaient possession du terrain pour évoluer vers le climax.

Les forêts de Cedrus libani qui occupent le versant Est du Djebel Alaouite ont subi le même sort. L'association typique du Cedrus libani (Cedretum libani) n'existe plus dans le Djebel Alaouite. Elle est remplacée par un groupement constitué d'arbres et d'arbustes héliophiles au milieu desquels le Cèdre se trouve disséminé par pieds isolés ou par petits ilôts.

Les forêts de sapin de Cilicie (Abies cilicica), bien que assez dégradées, sont mieux conservées que les forêts de Cèdres en raison de leur présence sur les versants Ouest et Nord-Ouest du Djebel Alaouite.

Enfin, l'homme a favorisé, par les défrichements et les exploitations des forêts, l'extension d'une essence forestière héliophile, colonisatrice des places vides, le Pinus brutia. Grâce à sa faculté de colonisation, cette essence s'est superposée dans beaucoup d'endroits à d'autres associations. Elle s'est installée dans l'association à Quercus calliprinos et Pistacia palaestina, association à Ceratonia siliqua et Pistacia Lentiscus, association à Quercus pseudocerris, etc.

\section{ChapITRE TROISIL̀ME}

CARACTERISTIQUES FLORISTIQUES ET PHYTOGEOGRAPHIQUES DU BAER-BASSIT ET DU DJEBEL ALAOUTTE

Dans ce chapitre, nous allons donner un aperçu sur les caractéristiques floristiques et phytogéographiques du Baer-Bassit et du Djebel Alaouite, ce qui nous permettra de situer ces deux régions 
par rapport aux régions floristiques et phytogéographiques du monde et montrer leur affinité avec les contrées qui leur sont limitrophes. D'autre part, cette étude nous servira de base pour l'étude de la végétation $\left({ }^{1}\right)$. La connaissance de la flore ${ }^{1}$ ) d'un pays est indispensable pour l'étude et la compréhension de sa végétation; elle est à la base même de cette étude.

\section{I — NOTIONS GENERALES FLORISTICO-PH YTOGEOGRAPHIQUES}

\section{Royaumes floraux}

Les classifications modernes des territoires floraux $\mathrm{du}$ monde sont représentées par les idées de ENGLER, RIKLI, DIELs, HaYEK. Ces idées peuvent être résumées dans la classification des systèmes floraux présentée par ENGLer et Diels dans la $11^{\circ}$ édition de "Syllabus der Pflanzenfamilien ", 1936.

Dans cette classification, les auteurs admettent quatre Royaumes ou Empires floraux pour la végétation terrestre du monde:

I. Royaume holarctique

II. Royaume paléotropical

III. Royaume néotropical

IV. Royaume océanique móridional (southern Oceanic)

Le Royaume holarctique qui nous intéresse plus particulièrement embrasse l'Europe, l'Afrique du Nord, la majeure partie de l'Asie et de l'Amérique du Nord. Cette grande division de la flore n'a pas une très grande importance pratique parce que la totalité ou la presque totalité de la flore d'un pays quelconque appartient généralement à un seul Royaume floral. Mais dans le cas d'un pays vaste comme la Syrie et qui possède des enclaves et des irradiations d'espèces appartenant à d'autres Royaumes

(1) La flore est une liste de plantes : étudier la flore d'un pays c'est étudier la liste des plantes qui y habitent. Dans l'étude de la végétation d'une région on traite des groupements physionomiques, des associations végétales, des relations des végétaux avec les conditions du milieu. 
floranx, cette notion nous permet de situer la flore de Baer-Bassit et du Djebel Alaouite par rapport à la flore des autres régions de Syrie et des pays voisins et en particulier ceux situés au Sud et à l'Est.

\section{Régions et Provinces phytogéographiques}

Les différentes parties des Royaumes floraux dont la flore et la végétation forment un ensemble naturel aussi bien au point de vue biologico-physionomique (expression des conditions du milieu) qu'au point de vue floristique (héritage des époques antérieures) sont appellées des Régions phytogéographiques.

BRAUN-Blanquet reconnaît six catégories de territoires régionaux (région, province, secteur, sous-secteur, district, sousdistrict). Son système est fondé sur une combinaison de caractéristiques floristiques et végétationnelles. Il a appliqué sa conception de "l'association végétale" et celle du groupement de ces associations en "unités hiérarchiques" plus élevées établi sur la base de la parenté revelée par les espèces de grande fidélité.

Les Régions phytogéographiques sont caractérisées par des conditions climatiques qui leur sont propres, et par de nombreuses communautés végétales climaciques. Du point de vue floristique, ces Régions sont caractérisées par des "endémiques" d'ordre systématique supérieur (Familles, tribus, genres), ex. : Région méditerranéenne, Région euro-sibérienne-boréo-américaine...

Les deux petites familles des Cnéoracées (deux espèces) et des Cynomoriacées (une espèce) sont spéciales à la Région méditerranéenne qui compte, en outre, trois tribus endémiques: celles des Romarins (Labiées), celle qui est constituée par le Coris monspeliensis (Primulacées) et enfin celle de l'Aphyllanthes monspeliensis de la famille des Liliacées.

Les genres endémiques sont au nombre de plusieurs centaines. Certains genres comme les Cistus, les Ononis, les Astragalus, les Thymus y offrent de très nombreuses espèces. On y compte plusieurs milliers d'espèces endémiques.

La notion de Région phytogéographique est assez concrète. La Région phytogéographique est en réalité l'unité la plus com- 
préhensive. L'unité inférieure qui est la Province, est aussi très intéressante pour le phytogéographe car, en principe, le climat qui règne dans une même Région phytogéographique n'est pass rigoureusement le même partout. Il existe souvent des nuances dans la durée de la saison sèche, de la saison pluvieuse, des températures maximales et minimales et qui ont le plus souvent une influence assez marquée sur les végétaux. C'est le cas du climat méditerranéen qui présente des nuances dans les parties occidentale et orientale de la Région méditerranéenne et que nous avons développées en détail dans la chapitre concernant le climat. La division de la Région méditerranéenne en Province occidentale et Province orientale est tout à fait justifiée, et nous pensons que cette justification est avant tout d'ordre "bioclimatique " que floristique.

Ces Provinces sont, bien entendu, caractérisées du point de vue floristique par des genres et des espèces endémiques et également par des communautés végétales climaciques qui leur sont propres, comme nous le verrons plus loin dans l'étude de la végétation du Baer-Bassit et du Djebel Alaouite et la comparaison de cette végétation avec celle du pourtour oriental de la Méditerranée.

EIG cite 170 plantes méditerranéo-orientales endémiques en Syrie et en Palestine ainsi que trois genres : Astoma DC., Cylotaxis Boiss., Pilgerochloa Eig. De ces considérations sur les Royaumes floraux et les Régions phytogéographiques une double et profonde différence entre ces deux sortes d'unités se manifeste:

1) Dans les Royaumes floraux, le facteur " température" intervient principalement, tandis que les Régions phytogéographiques sont déterminées principalement par la combinaison des deux facteurs : "température et humidité " et, dans le cas de la Région méditerranéenne qui nous intéresse plus particulièrement, nous pensons que le facteur édaphique peut jouer un rôle non négligeable.

2) Les Royaumes floraux reposent presque exclusivement sur la flore en accentuant l'argument historique; les Régions phytogéographiques, au contraire, combinent à la fois la flore et la végétation.

Nous déduisons de ce qui précède que le Baer-Bassit et le 
Djebel Alaouite appartiennent au Royaume floral holarctique et à la Province orientale de la Région méditerranéenne (ou à la Méditerranée orientale).

\section{Element}

Le terme "élément" a été, en général, employé par différents auteurs et dans les sens les plus divers. Dans cette étude, nous utilisons ce terme dans le sens développé par EıG (1933) et que nous pourrons définir comme suit:

Chaque Région phytogéographique bien délimitée au point de vue physique possède une flore et une végétation plus ou moins individualisées, c'est-à-dire dont beaucoup de traits lui sont propres. Cette flore et cette végétation sont ainsi son expression, son incarnation phytogéographique. Ces expressions particulières des Régions phytogéographiques naturelles, les sippes et les groupements végétaux spéciaux qui reflètent les conditions écologiques particulières forment ensemble "l'élément" de chaque Région.

Ainsi, à la Région méditerranéenne correspond un élément méditerranéen ; à la Région euro-sibérienne-boréo-américaine, un élément euro-sibérien-boréo-américain ; à la Région irano-touranienne, un élément irano-touranien, etc.

EIG subdivise l'élément méditerranéen en cinq sous-éléments principaux que nous résumons dans ce qui suit :

3.1. Sous-élément omni-méditerranéen : e'est un sous-élément de liaison de tout le pourtour de la Méditerranée, exception faite pour le sud de la Palestine, l'Egypte et l'Est de la Lybie. Certaines espèces appartenant à la Région méditerranéenne et habitant en plus de cette Région, les montagnes de la Région Irano-touranienne voisine (Kurdistan, Iran, Transcaucasie) sont classées dans ce groupe mais sont appelées sub-méditerranéennes.

3.2. Sous-élement méditerranéo-oriental : les espèces de ce groupe habitent une partie ou toute la Région méditerranéenne orientale. EIG considère les "Balkans" comme limite naturelle de ce sous-élément pour le Nord de la Méditerranée et "Barka" comme sa limite naturelle en Afrique du Nord. Ce terme sub- 
méditerranéo-oriental est donné aussi aux espèces qui habitent la Méditerranée orientale et se rencontrent aussi dans les montagnes de la Région Irano-touranienne voisine (Irano-anatolienne).

3.3. Sous-élément méditerranéo-occidental : les espèces de ce groupe habitent une partie ou toute la Méditerranée occidentale. Généralement, la Méditerranée occidentale est étendue jusqu'à l'Italie. Du côté de l'Afrique du Nord, la question a été plus discutée. EIG considère Barka en Cyrénaique comme appartenant déjà à la Méditerranée orientale. En effet, un certain nombre d'espèces importantes et bien caractéristiques de la Méditerranée orientale se retrouvent en Cyrénaique comme : Cupressus sempervirens, Ceratonia siliqua, Salvia triloba, Teucrium divaricatum, Teucrium brevifolium. Plus important encore est la présence du Poterium spinosum, l'une des plantes les plus caractéristiques de la Méditerranée orientale. Cette séparation est faite, par conséquent, sur des bases essentiellement floristiques. Elle comporte done trop d'imprécisions car la flore actuelle est le résultat de l'histoire floristique des régions considérées. Nous pensons que l'étude détaillée des bioclimats permettra de séparer la Méditerranée occidentale de la Méditerranée orientale sur des bases beaucoup plus naturelles.

3.4. Sous-élément méditerranéo-septentrional : les espèces de ce groupe habitent le Nord de la Méditerranée.

3.5. Sous-élément méditerranéo-méridional : les espèces de ce groupe habitent le Sud de la Méditerranée. Il est utile de considérer séparément les sous-éléments sud et nord méditerranéens. En effet, les pays se trouvant des deux côtes de la Méditerranée mais dans la même subdivision orientale ou occidentale ont entre eux plus d'affinité floristique que les pays situés du même côté mais dans les subdivisions différentes. Par exemple, l'élément méditerranéen de la France a plus d'affinité avec l'élément méditerranéen d'Algérie qu'avec celui de la Grèce.

l'élément méditerranéen, avec ses 5 sous-éléments, dominent dans le Baer-Bassit et le Djebel Alaouite, comme nous verrons un peu plus loin.

Il est quelquefois délicat de dire à quel élément appartient une de nos espèces. Ceci est dû à la connaissance insuffisante de nos espèces. Ces cas deviennent en général assez nombreux au 
Moyen-Orient où l'étude de la systématique des plantes et de leur écologie n'est pas suffisamment avancée. En effet, pour déterminer à quel elément appartient une espèce, il est tout à fait insuffisant de connaître seulement sa répartition géographique dans ses traits généraux, mais il faut avoir aussi des données sur sa fréquence, sa vitalité, son importance dans les associations végétales, etc. Dans d'autres cas, l'incertitude provient du fait que les plantes, paraissant, à première vue, appartenir morphologiquement à la même unité taxonomique, ont souvent des exigences écologiques différentes. C'est seulement quand ces différences écologiques sont bien connues que ressortent aussi des différences morphologiques, sinon saillantes, du moins tout à fait constantes; on est alors convaincu d'avoir affaire à des unités taxonomiques distinctes. La question se complique davantage done avec les écotypes, unités beaucoup plus difficiles à déterminer. Nous sommes convaincu que la connaissance plus approfondie des unités taxonomiques dans les temps futurs apportera beaucoup de précisions à nos travaux en Phytogéographie et Ecologie végétale. Notre étude ressent donc les imprécisions et les lacunes existant dans la connaissance du matériel végétal sur lequel elle est fondée.

\section{Enclaves}

Dans une Région phytogéographique donnée, on trouve souvent certaines étendues où règnent des conditions écologiques spéciales et où vivent des plantes appartenant à un élément étranger et n'existant pas en dehors de ces étendues. Ces plantes peuvent pénétrer dans les associations de l'élément de base où former des groupements spéciaux. On parle alors des enclaves des territoires étrangers et par conséquent des enclaves des éléments. Historiquement ces enclave sont presque toujours des restes d'anciennes expansions de leurs éléments mères. C'est le cas, par exemple, des enclaves septentrionales ou nordiques, qui existent dans l'Amanus, le Djebel Alaouite et que nous traiterons un peu plus loin. 


\section{Irradiations}

On trouve souvent dans une Région phytogéographique déterminée des plantes appartenant à des éléments étrangers sans que les territoires où ces plantes poussent offrent des conditions écologiques nettement spéciales et sans que le nombre de ces plantes étrangères soit considérable. Dans ces conditions, on ne peut pas parler de vraies enclaves, on parle alors " d'irradiations".

Ces irradiations sont quelquefois des restes des expansions anciennes de leurs éléments-mères qui ont réussi à conserver leur place après le changement des conditions climatiques et continué à vivre avec les éléments nouveaux survenus après ce changement, sans former toutefois de vraies enclaves. Mais les irradiations résultent, le plus fréquemment, du fait qu'une région naturelle possède vers sa limite des conditions un peu spéciales, permettant à un bon nombre d'espèces étrangères de pénétrer plus ou moins profondément dans cette région. Les irradiations peuvent également dépendre de certaines conditions climatiques spéciales qui sont liées aux changements saisonniers. Par exemple, la saison pluvieuse bivernale dans la Région méditerranéenne favorise la pénétration des plantes septentrionales dans cette Région.

\section{Plantes de liaison}

Chaque Région naturelle possède, en dehors de son élément qui lai est propre, des enclaves et des irradiations, des éléments étrangers, un certain nombre de plantes plus ou moins largement représentées dans cette Région comme dans des Régions voisines et croissant pour la plupart ça et là dans des conditions écologiques plus ou moins propres à chacune de ces Régions.

Bien que ces plantes soient surtout représentées par des plantes rudérales, ségétales et hydrophiles, leur signification phytogéographiques reste certaine.

D'après EIG, $88 \%$ des espèces rudérales et ségétales palestiniennes sont particulières aux Régions méditerranéenne et iranotouranienne ; ou si elles dépassent ces deux Régions et se trouvent aussi ailleurs, par exemple dans la Région euro-sibérienne-boréo- 
américaine, elles montrent en dehors de ces deux régions des exigences écologiques particulières, qui révèlent leurs affinités spéciales aux Régions méditerranéenne et irano-touranienne. Une petite partie, seulement $12 \%$, des espèces rudérales et ségétales de la Palestine sont des ubiquistes ou cosmopolites et montrent ainsi une vaste amplitude écologique.

On peut donc dire, que malgré la présence d'un grand nombre de plantes ségétales, rudérales et hydrophiles, les espèces de ce groupe sont apparemment aussi des indicatrices des affinités écologiques entre Régions naturelles voisines. Plus les climats de ces Régions voisines sont similaires et plus importantes sont les plantes qui leur sont communes.

Eig désigne ces plantes communes, indicatrices des affinités écologiques, floristiques et phytosociologiques des éléments ou des Régions phytogéographiques naturelles sous le nom de "plantes de liaison".

La délimitation des plantes de liaison exige une connaissance approfondie de la végétation et des conditions écologiques des Régions étudiées. Cette connaissance est loin d'être complètement acquise pour la Région méditerranéenne et en particulier pour sa partie orientale où la systématique n'est pas très avancée; beaucoup de massifs étant encore mal connus et où les études de la végétation ne font que débuter.

\section{Plantes plurirégionales ou cosmopolites ou ubiquistes}

On donne le nom de plantes ubiquistes, cosmopolites ou plurirégionales à des plantes d'une Région naturelle dont l'aire géographique embrasse plusieurs Régions appartenant à des Empires floraux différents et n'ayant entre elles que peu ou pas de traits communs au point de vue écologique et n'étant pas limitrophes géographiquement.

Signalons qu'un nombre très restreint seulement de ces plantes est réellement cosmopolite. Il est done préférable d'utiliser le terme "plantes plurirégionales" au lieu de "plantes cosmopolites ou ubiquistes ".

Dans ce qui suit, nous allons appliquer ces notions aux régions 
du Baer-Bassit et du Djebel Alaouite. Le classement des espèces dans une des catégories de ces subdivisions a été fait à la suite des travaux que nous avons poursuivis dans ces régions depuis 1956 et d'après les travaux de A. Eig sur la Palestine (Les eléments et les groupes phytogéographiques auxiliaires dans la flore palestinienne, 1931). Pour la répartition géographique des espèces dans le Proche-Orient, nous avons consulté les Flores suivantes : F'lora Orientalis de E. BoIssIER, 1867-88; Flore du Liban et de la Syrie de L. Bouloumoy, 1930 ; Flore Libano-Syrienne de J. THitBAU', 1936 et 1953 ; Fllora of Syria, Palestine and Sinai de G. E. PosT and J. E. DINSMORE, 1932-33; Flora of Egypt de V. TäcKHoIM et M. DRAR, 1941-50-54; Flore de l'Iran de M. PARSA, 1949-1960.

II - LES ELEMENTS MEDITERRANEENS DANS LE BAER-BASSIT ET LE DJEBEL ALAOUTTE

\section{Sous-6lément omni-méditerranéen (Om-Med)}

Parmi les espèces appartenant à ce sous-élément nous citerons les suivantes :

\section{a. Espèces omni-méditerranéennes}

\author{
Anacardiacées \\ Pistacia Lentiscus L. \\ P. Terebinthus L. \\ Aristolochiacées \\ Aristolochia altissima Desf. \\ Borraginacées \\ Anchusa hybrida Ten. \\ Echium plantagineum L. \\ Caryophyllacées \\ Paronychia argentea Lam. \\ P. capitata Lam. \\ Silene Behen L. \\ S. fuscata Link \\ S. muscipula L. \\ S. nocturna $L$. \\ S. sedoides Poir.
}

Chenopodiacées Atriplex Halimus L.

Cistacées Cistus villosus $\mathbf{L}$.

i Helianthemum lavandulifolium Mill. Composées Ambrosia maritima L. Asteriscus aquaticus Leas. Carlina corymbosa L.

C. lanata L. Catananche lutea $L$.

Chrysanthemum coronarium L. C. Myconis L.

Conyza linifolia (Willd.) Tăckh. (= Erigeron linifolius Willd., E. crispus Pourr.) 
Crepis bulbosa (L.) Tausch

Hyoseris scabra $\mathbf{L}$.

Inula graveolens (L.) Boiss.

I. viscosa (L.) Ait.

Senecio leucanthemifolius Poir.

Crassulacées

Sedum altissimum Poir.

Cruciferes

Arabis verna (L.) DC.

Matthiola tricuspidata L.

Cyperacées

Cyperus schoenoides Griseb.

Enicacées

Arbutus Unedo $L$.

Euphorbiacées

Euphorbia terracina L.

Fumariacées

Fumaria agraria Lag.

Geraniacées

Erodium laciniatum (Cav.) Willd.

E. romanum (L.) willd.

Graminées

Aegilops ovata L.

Bromns alopecurus Poir.

B. fasciculatis Presl.

Catapodium loliaceum Huds.

C. tuberculosum Moris

Gastridium seabrum Presl

Oryzopsis miliacea (L.) Asch. et Schweinf.

Phalaris brachystachys Link

Scleropoa maritima (L.) Parl.

Iridacées

Romulea Bulbocodium (L.) Seb. et Mauri

Labiées

Lavandula Stoechas $\mathbf{L}$.

Micromeria graeca (L.) Benth.

M. juliana (L.) Benth.

M. nervosa (Desf.) Benth.

Molucella spinosa $\mathrm{L}$.

Prasium majus $L$.

Sideritis romana $\mathbf{L}$.

Thymus capitatus (L.) Link et Hoffm.

Légumineuses

Astragalus baeticus $\mathrm{L}$.

A. Epiglottis L.

Bisserula Pelecinus L.

Calycotome villosa Link
Doryenium rectum (L.) Ser.

Hippocrepis multisiliquosa L. Lathyrus Ochrus (L.) DC.

Lotus edulis L.

L. ornithopodioides L.

Lupinus angustifolius $\mathrm{L}$.

L. hirsutus $\mathrm{L}$.

Medicago tuberculata Willd.

Melilotus infestus Guss.

M. sulcatus Desf.

Ononis alopecuroides L.

0 . hirta Desf.

o. Natrix L.

o. ornithopodioides L.

o. pubescens $\mathrm{L}$.

o. variegata $L$.

Physanthyllis tetraphylla (L.) Boiss.

Liliacées

Asparagus acutifolius L.

A. aphyllus $\mathrm{L}$.

Asphodelus microcarpus Salzm. et Viv.

Muscari maritum Desf.

Urginea maritima (L.) Baker

Lythracées

Lythrum flexuosum Lag.

Oléacées

Phillyrea media L.

Ombelliferes

Erygium Barrelieri Boiss.

Pseudorlaya pumila (L.) Grande (= Orlaya pumila [L.] Hal., 0 .

Orchidacées

maritima [Gou.] Koch)

Orehis fusca Link

o. papilionacea $\mathrm{L}$.

o. tenthredinifera Willd.

Pinacées

Pinus halepensis Mill.

Plombaginacées

Statice virgata Willd.

Polygonacées Emex spinosus (L.) Campd.

Rumex bucephalophorus $L$.

Renonculackes

Clematis cirrhosa $L$.

Rhamnacées

Rhamnus Alaternus L.

Rosacées

Poterium verrucosum Ehrenb. 


\section{Rubiacées}

Crucianella latifolia $L$.

Galium murale (L.) All.

Santalacées

Osyris alba $\mathrm{L}$.

\author{
Solanacées \\ Hyoscyamus albus $\mathrm{L}$. \\ Lycium europaeum $L$. \\ Urticacées \\ Urtica membranacea Poir.
}

\section{b. Espèces sub-méditerranéennes}

Amaryllidées

Narcissus Tazetta L.

Pancratium maritimum L.

Anacardiacées

Rhus coriaria $\mathrm{L}$.

A pocynactes

Nerium oleander L.

Caprifoliacées

Lonicera estrusca Santi

Caryophyllacées

Silene gallica L.

Cistacées

Cistus salviaefolius L.

Fumana arabica (L.) Spach

Oomposées

Atractylis cancellata $\mathbf{L}$.

Bellis annua L.

B. silvestris Cyrill.

Chrysanthemum segetum $\mathbf{L}$.

Diotis maritima (L.) Sm.

Inula crithmoides $L$.

Picris Sprengeriana (L.) Lam.

Scolymus maculatus L.

Coniferes

Juniperus oxycedrus L.

Convolvulacées

Convolvulus cantabrica L.

Cruciferes

Raphanus Raphanistrum L.

(Daphnéacées) (Thymélées)

Thymelaea hirsuta (L.) Endl.

Discoreacées

Tamus communis $\mathrm{L}$.

Euphorbiacées

Euphorbia exigua L.

E. Paralias $L$.

E. peplis $\mathbf{L}$.

E. peploides Gouan

Mercurialis annua $\mathbf{L}$.

Fumariacées

Fumaria capreolata $L$.
Geraniacées

Geranium lucidum L.

G. purpureum Vill.

Araminées

Briza minor I.

Chrysopogon gryllus Trin.

Corynephorus articulatus (Desf.) Beauv.

Cynosurus echinatus L.

Echinaria capitata (L.) Desf.

Gastridium lendigerum Gaud.

Gaudinia fragilis (L.) Beauv.

Holcus annuus Salzm.

Lagurus ovatus $\mathbf{L}$.

Lolium multiflorum Lam.

Monerma cylindrica (Willd.) Coss. et Dur.

Vulpia membranacea (L.) Link (= V. uniglumis [Sol.] Dum.)

Hypericacées

Hypericum erispum L.

Labiées

Melissa officinalis $\mathbf{L}$.

Salvia verbenaca $\mathbf{L}$.

Lauracées

Laurus nobilis L.

Légumineuses

Anagyris foetida $\mathrm{L}$.

Hippocrepis unisiliquosa L.

Medicago ciliaris Willd.

M. littoralis Rohde

M. marina $L$.

M. seutellata All.

Melilotus messanensis (L.) Degf.

Onobrychis Caput-galli (L.) Lam.

Ononis breviflora DC.

Ononis mitissima $\mathrm{L}$.

Ononis serrata Forsk.

Ornithopus compressus L.

Pisum elatius M. Bieb.

Scorpiurus subvillosa $\mathbf{L}$. 
Spartium junceum L.

Trifolium Cherleri L.

T. spumosum $\mathbf{L}$.

T. stellatum $\mathrm{L}$.

Vicia hybrida L.

Liliacées

Asphodeline lutea (L.) Rehb.

Gagea arvensis (Prs.) Dum.

Ruscus aculeatus $\mathrm{L}$.

Scilla autumnalis L.

Linacées

Linum angustifolium Huds.

Malvacées

Lavatera trimestris L.

Myrtacées

Myrtus communis L.

Oléacées

Olea europaea $L$.

Ombelliféres

Foeniculum piperitum Presl

Physocaulos nodosus (L.) Tausch
Ridolfia segetum Moris

Smyrnium olusatrum L.

Orchidacées

Aceras pyramidalis Rchb.

Orchis saccatus Ten.

Polygalacées

Polygala monspeliaca L.

Renonoulacées

Adonis antumnalis Boiss.

Clematis Flammula L.

Ranunculus lateriflorus DC.

Rubiacées

Putoria calabrica (L. f.) Pers.

Santalacées

Thesium humile Vahl

Scrofulariacées

Linaria Pelisseriana L.

Trixago apula Stev.

Urticacées

Cynocrambe prostrata Gaertn.

Le sous-élément omni-méditerranéen est donc bien représenté dans le Baer-Bassit et le Djebel Alaouite. Les espèces qui en font partie sont très familières dans le paysage de ces régions. Certaines d'entre elles se montrent fidèles aux associations végétales les plus caractéristiques comme nous verrons plus loin. Citons ici : Pistacia Lentiscus qui constitue avec le Ceratonia siliqua le Ceratonieto-Lentiscetum, association caractéristique des régions littorales ; Cistus salviaefolius, Cistus villosus, très répandus dans les associations de dégradation; Phillyrea media, Rhamnus alaternus, Laurus nobilis, Olea europaea, Ruscus aculeatus, Spartium junceum, Calycotome villosa, Lonicera etrusca, Thymus capitatus, Juniperus Oxycedrus, etc.

\section{Sous-6lément mediterranéo-oriental (Est-Med)}

Parmi les espèces qui appartiennent à ce sous-élément nous citerons les suivantes :

\section{a. Espèces méditerranéo-orientales}

Aceracées

Acer syriacum Boigs. et Gaill.
Amaryllidées

Pancratium parviflorum Dec. 


\section{Anacardiacées}

Pistacia palaestina Boiss.

Aristolochiacées

Aristolochia parvifolia Sibth. et Sm.

Aroidées

Arum hygrophyllum Boiss.

Borraginacées

Anchusa aggregata Lehm.

Echium glomeratum Poir.

Heliotropium Bovei Boiss.

H. villosum Willd.

Nonnea obtusifolia (Willd.) R. et S.

Onosma frutescens Lam.

o. giganteum Lam.

Symphytum palestinum Boiss.

Campanulacées

Campanula retrosa Labill.

C. stellaris Boiss.

Michauxia campanuloides L'Hérit.

Caprifoliacées

Lonicera orientalis Lam.

Caryophyllacées

Silene damascena Boiss. et Gaill.

S. juncea Sibth.

S. succulenta Forsk.

Oomposées

Anthemis leucanthemifolia Boiss. et Bl.

A. tripolitana Boiss. et $\mathrm{Bl}$.

Atractylis comosa Sieb.

Cardopatium corymbosum (L.) Pers.

Centaurea araneosa Boiss.

C. Crocodylium $\mathbf{L}$.

C. cyanoides Berggr. et Wahlenb.

Chamaepeuce Alpini Jaub. et Sp.

Crepis aculeata (DC.) Boiss.

C. hierosolymitana Boiss.

Cynara syriaca Boiss.

Helichrysum sanguineum (L.) Boiss.

Lactuca cretica Desf.

Lagoseris bifida (Vis.) F. et $\mathbf{M}$.

Coniferes

A bies cilicica (Ant. et Ky) De Tehih.

Cedrus Libani A. Rich.

Juniperus drupacea Lab.

Convolvulacées

Convolvulus coelesyriacus Boiss.

C. secundus Desv.

Cruciferes

Carpoceras oxyceras Boiss.
Enarthrocarpus arcuatus Labill.

Erophila setulosa Boiss. et Bal.

Lepidium spinosum $L$.

Malcolmia chia (Lam.) DC.

Ochtodium aegyptiacum (L.) DC.

Cupulifères

Quercus Aegilops L.

Q. Calliprinos Webb

Q. Cerris L.

Q. Ehrenbergii Ky.

Q. microphylla Thieb

Q. pseudocerris Boiss.

Cypéracées

Carex mediterranea C.B.Cl.

Daphnacées (Thymélées)

Lygia Aucheri (Meisn.) Boiss.

Dipsacées

Cephalaria joppensis (Spreng.) Coult.

Scabiosa prolifera L.

Ephedracées

Ephera campylopoda C.A.M.

Ericacées

Arbutus Andrachne L.

Euphorbiacées

Euphorbia arguta Soland.

E. aulacosperma $\mathbf{L}$.

E. berythea Boiss.

E. Reuteriana Boiss.

E. thamnoides Boiss.

Fumariacées

Ceratocapnos palaestina Boiss.

Fumaria anatolica Boiss.

F. judaica Boiss.

F. macrocarpa Parl.

Graminées

Bromus flabellatus (Hack.) Boiss.

B. syriacus Boiss. et $\mathrm{Bl}$.

Hypericacées

Hypericum serpyllifolium Lam.

\section{Iridacées}

Crocus hyemalis Boiss. et Bl.

C. ochroleucus Boigs. et Gaill.

C. vitellinus Vahl.

Labiées

Calamintha incana (Sibth. et Sm.) Boiss.

Lamitum moschatum Mill.

Micromeria serpyllifolia (M. Bieb.) Boiss.

Nepeta cilicica Boiss. 
Phlomis viscosa Poir.

Salvia hierosolymitana Boiss.

S. judaica Boiss.

S. triloba L. f.

Sideritis pullulans Vent.

Stachys arabica Hornem.

S. neurocalycina Boiss.

S. viticina Boiss.

Légumineuses

Astragalus berythaeus Boiss.

Ceratonia siliqua $\mathbf{L}$.

Genista acanthoclada DC.

G. sphacelata Dec.

Lathyrus blepharicarpus Boiss.

L. hierosolymitanus Boiss.

L. marmoratus Boiss. et Bl.

Lotus palaestinus $\mathbf{L}$.

(= Tetragonolobus palaestinus Boiss.)

Lupinus pilosus $\mathbf{L}$.

L. Termis Forsk.

Medicago Blancheana Boiss.

Trifolium comosum Labill.

T. elypeatum $\mathrm{L}$.

T. dichroanthum Boiss.

T. erubescens Fenzl.

T. globosum $\mathrm{L}$.

T. nervulosum Boiss, et Heldr.

T. scutatum Boiss.

T. xerocephalum Fenzl

Trigonella cylindracea Desv.

T. spinosa $\mathrm{L}$.

Vicia galeata Boiss.

Liliacées

Allium curtum Boiss. et Gaill.

Bellevalia longipes Post

B. trifoliata (Ten.) Kunth.

Fritillaria libanotica Boiss.

Ornithogalum lanceolatum Labill.

Malvacées

Althaea acaulis Cav.

Malva oxyloba Boiss.

Ombelliferes

Anthriscus lamprocarpa Boiss.
Bupleurum nodiflorum $S$. et $\mathrm{S}$. Cachrys goniocarpa Boiss.

Chaetosciadium trichospermum (L.) Boiss.

Daucus Broteri Ten.

D. littoralis Sibth. et Sm.

Eryngium falcatum Laroch.

Exoacantha heterophylla Lab.

Ferulago syriaca Boiss.

Oenanthe prolifera $L$.

Tordylium syriacum $L$.

Primulacées

Cyclamen persicum Mill.

Renonoulacées

Delphinium ithaburense Boiss.

Helleborus vesicarius Auch.

Résédacées

Reseda orientalis Boiss.

Rhamnacées

Rhamnus palaestina Boiss.

R. punctata Boiss.

Rosacées

Poterium spinosum $\mathrm{L}$.

Prunus ursina Ky.

Rosa phoenicea Boiss.

Rubiackes

Crncianella macrostachya Boiss.

Galium aureum Vis.

G. hierosolymitanum $L$.

G. pisiferum Boiss.

Saxifragacées

Saxifraga hederacea $L$.

Serofulariacées

Scrophularia macrophylla Boiss.

S. Michoniana Coses. et Kral.

Verbascum caesareum Boiss.

V. tripolitanum Boiss.

Veronica leiocarpa Boiss.

V. syriaca Roem. et Sch.

Styracacées

Styrax offieinale L.

Tamaricacées

Tamarix tetrandra Pall.

\section{b. Espèces subméditerranéo-orientales}

\section{Aroidées}

Arum Dioscoridis Sibth. et Sm.
Eminium spiculatum (Blume) Ktze (= Helicophyllum crassipes Schott) 


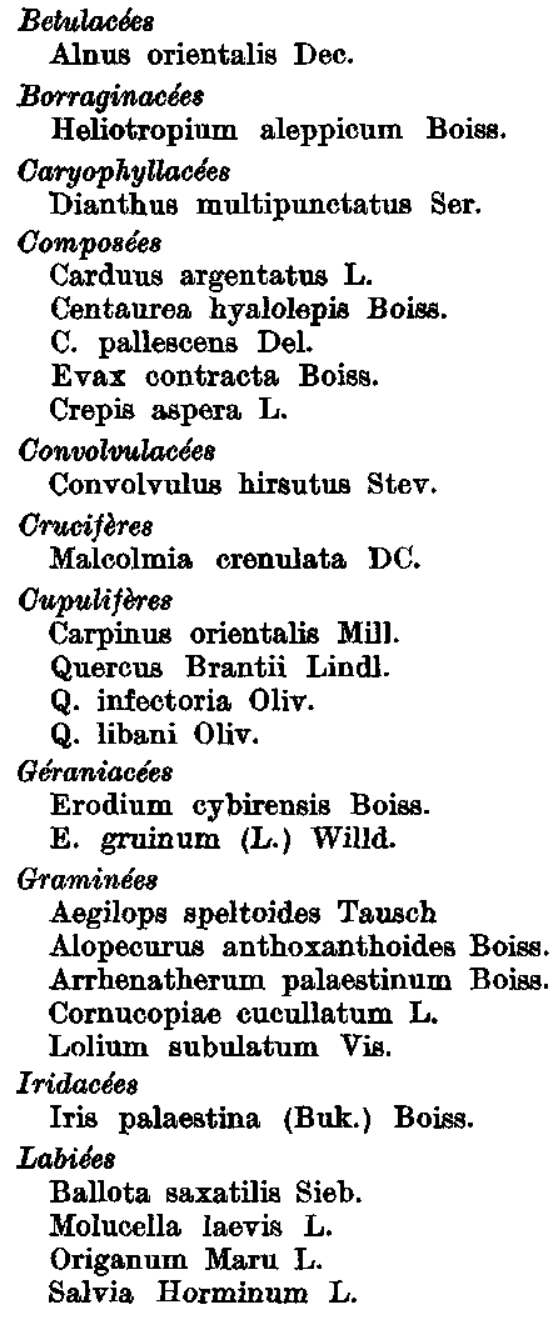

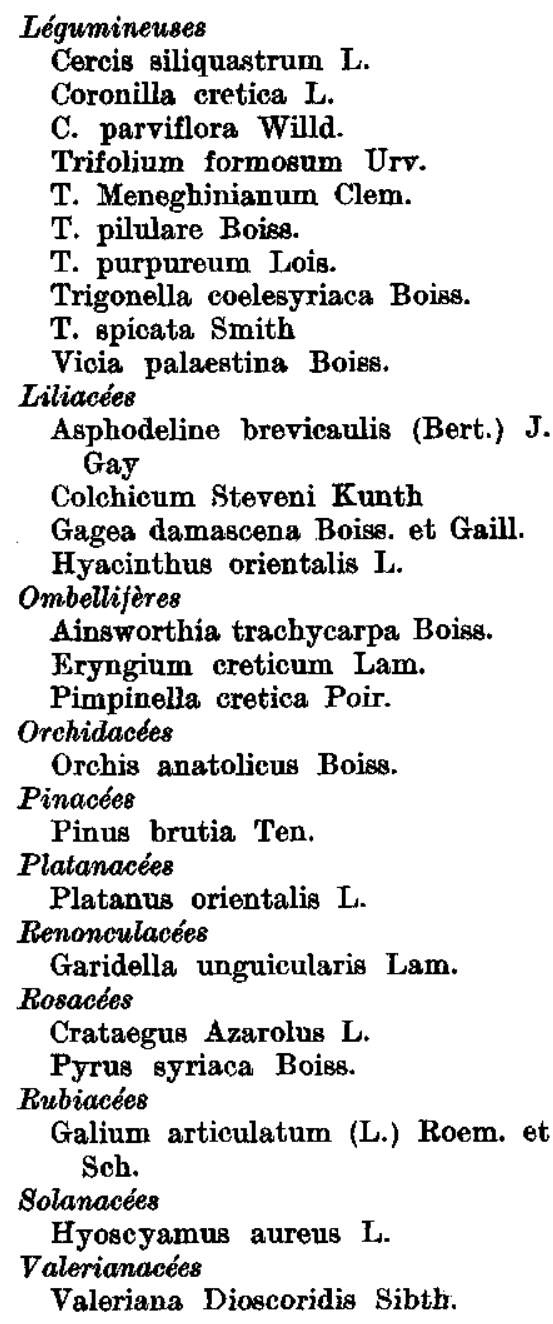

Légumineuses

C. parviflora Willd.

Trifolium formosum Urv.

Trigonella coelesyriaca Boiss.

T. spicata Smith

Vicia palaestina Boigs.

Asphodeline brevicaulis (Bert.) J. Gay

Gagea damascena Boiss. et Gaill.

Hyacinthus orientalis $\mathbf{L}$.

Ombellifères

Ainsworthia trachycarpa Boiss.

Eryngium creticum Lam.

Orchis anatolicus Boiss.

Pinus brutia Ten.

Platanacées

Platanus orientalis L.

Renonculacées

Garidella unguicula

Crataegus Azarolus L.

Pyrus syriaca Boiss.

Rubiacées

Galium articulatum (L.) Roem. et

olanacées

alerianacées

Valeriana Dioscoridis Sibth.

Les espèces les plus caractéristiques de la Méditerranée orientale appartiennent à ce groupe, comme: Poterium spinosum, Ceratonia siliqua, Salvia triloba, etc. Certaines d'entre elles constituent des association climaciques caractéristiques de la Méditerranée orientale. Parmi ces espèces nous pouvons citer : Cedrus Libani, Abies cilicica, Quereus calliprinos, Quercus pseudocerris, Pinus brutia. D'autres enfin sont de première importance dans le Baer- 
Bassit et le Djebel Alaouite tant par leur fréquence que par leur présence dans les associations caractéristiques comme: Arbutus Andrachne, Pistacia palaestina, Styrax officinale, Acer syriacum, Platanus orientalis, Rhamnus punctata, Quercus infectoria, Pyrus syriaca, Prunus ursina, Thymbra spicata, Rubia Aucheri, etc. Nous voyons donc que la plus grande partie des arbres et arbrisseaux qui jouent un rôle important dans les forêts, garrigues et maquis du Baer-Bassit et du Djebel Alaouite appartient à l'élément méditerranéo-oriental.

\section{Sous-elément méditerranéo-occidental (Ouest-Méd.)}

Parmi les espèces de ce groupe nous rencontrons dans le BaerBassit et le Djebel Alaouite les suivantes :

Légumineuses

Lupinus reticulatus Desv. var. philisteus Boiss.

\section{Labiées}

Stachys arvensis $\mathbf{L}$.

\section{Sous-6́lément méditerranéo-septentrional (Nord-Med.)}

Parmi les espèces de ce groupe nous citerons les suivantes:

a. Espèces méditerranéo-septentrionales

\section{Liliacées}

Allium trifoliatum Cyrill.

Scilla hyacynthoides I.

b. Espèces sub-méditerranéo-septentrionales

\section{Gentianaéces}

Chlora perfoliata $L$.

Renonculacées

Paeonia corallina Retz.

Rosacées

Rubus tomentosus Borkh. 


\section{Sous-6́lément méditerranéo-méridional (Sud-Med.)}

\section{Borraginacées}

Echium sericenm Vahl

Convolvulacées

Labiées

Convolvulus tricolor L. (subspontané q)

Marrubium Alysson L.

Ombelliferes

Daucus aureus Desf.

Ce sous-élément est très peu représenté dans le Nord-Ouest de la Syrie. Il est un peu plus représenté au Liban et en Palestine (EIG en compte 14 espèces en Palestine).

\section{III - LES ENCLAVES}

\section{a. Les enclaves nordiques}

Comme nous le verrons plus loin, les périodes pluviales du Quaternaire sont à l'origine de ces enclaves qu'on trouve cantonnées dans la partie supérieure du Djebel Alaouite (dans la région de Slenfé et du Nabi Matta) au milieu d'une flore typiquement méditerranéenne. Parmi ces espèces nordiques nous pouvons citer :

Cerasus Mahaleb $\mathrm{L}$.

Corylus Avellana L. (subspontané q)

Daphne oleoides Schreb.

Inula Conyza DC.

Lapsana communis L. (dans le Cassius ?)

Sorbus torminalis Crantz

Sorbus umbellata (Desf.) var. flabellifolia (Spach) C. K. Schn. (= Sorbus Aria Crantz ssp. graeca (Lodd.)

Staphylea pinnata $\mathrm{L}$.

Evonymus latifolius (L.) Mill. (cité par PABot, 1957).

Ces enclaves sont plus importantes encore dans l'Amanus qui est situé au Nord du Baer-Bassit et qui s'élève à $2500 \mathrm{~m}$ d'altitude. Ses pluies sont plus régulières et la fraicheur y est entretenue par 
les brouillards qui s'installent fréquemment sur les hauteurs quand la brise souffle de la mer et débordent même sur le versant oriental. Les crêtes sont occupées par la neige pendent cinq mois de l'année.

Parmi les espèces nordiques de l'Amanus qui ne pénètrent pas le Baer-Bassit et le Djebel Alaouite nous pouvons citer :

Acer campestre $L$.

Alnus incana $\mathbf{L}$.

Asperula odorata L.

Asplenium septentrionale $\mathbf{L}$.

Atropa Belladonna $L$.

Circaea lutetiana L.
Fagus silvatics L. var. orientalis (Lipsky)

Fragaria vesca L.

Ilex aquifolium $\mathrm{L}$.

Lathraea squamaria $L$.

Sanicula europaea L.

Taxus baccata L.

\section{b. Les enclaves saharo-sindiennes}

Ces enclaves sont localisées surtout sur les dunes du littoral. Parmi ces espèces nous citerons :

Ammochloa Palaestina Boiss. (signalée comme existante sur littoral syrien par EIG)

Artemisia monosperma Del.

Citrullus colocynthis (L.) Schrad.

Echichilon fruticosum Desf.

Neurada procumbens $L$.

Le nombre de ces espèces saharo-sindiennes augmente au fur et à mesure que nous descendons vers le sud et devient très important sur le littoral palestinien.

Sur les dunes à côté de Beyrouth (Liban) on rencontre déjà Retama Raetam (Forsk.) Webb, Aristida Forskalei Tausch., Rumex oceultans Sam. (Rumex lacerus Balb.), espèces typiquement saharosindiennes.

La présence de ces espèces au milieu de l'élément méditerranéen est curieuse ; mais si nous examinons leurs lieux de répartition, nous constaterons qu'ils sont liés à la présence du sable sur le littoral. Les sols sablonneux en effet leur assurent des conditions de chaleur leur rappelant leur pays d'origine.

Le point de départ de l'infiltration de ces plantes sur le littoral méditerranéen est facile à fixer. Les dunes maritimes de la Palestine méditerranéenne sont reliées au sud aux dunes et 
aux sables du Negueb, qui ont une communication directe avec l'intérieur du Sinaï. Ceci explique le nombre plus important de ces plantes en Palestine (EIG en cite 28).

\section{IV - LES PLANTES DE LAISON}

\section{Plantes de liaison méditerraneo-irano-touraniennes (Liaison Med.-Ir.-Tour.)}

Parmi les espèces de ce groupe nous citerons les suivantes:

\section{a. Espèces méditerranéo-irano-touraniennes}

\section{Borraginacées}

Cynoglossum creticum Mill.

Campanulacées

Campanula Erinus L.

Specularia falcata (Ten) A. DC.

Caryophyllacées

Herniaria cinerea DC.

Cerastium dichotomum $\mathbf{L}$.

Polycarpon alsinifolium (Biv.) DC.

Silene conoidea $L$.

S. longipetala Vent.

\section{Cistacées}

Helianthemum salicifolium Mill.

\section{Composées}

Carduus pycnocephalus $\mathrm{L}$.

Crupina Crupinastrum (Moris)Vis.

Geropogon glabrum L.

Hedypnois cretica (L.) Willd.

Notobasis syriaca (L.) Cass.

Pallenis spinosa (L.) Cass.

Rhagadiolus stellatus (L.) Willd.

Silybum marianum (L.) Gaertn.

Sonchus glaucescens Jord.

S. maritimus $\mathrm{L}$.

Urospermum picroides (L.) F.W. Schmidt.

Oonvolvulacées

Cuscuta monogyna Vahl

Cmucifères

Brassica Tournefortii Gouan
Diplotaxis erucoides (Torner) DC. Sinapis alba $\mathbf{L}$.

Cucurbitacées

Ecballium Elaterium (L.) Rich.

Cypéracées

Cyperus distachyus All.

Euphorbiacées

Chrozophora tinctoria (L.) Raf.

Géraniacées

Erodium malacoides (L.) Willd.

Geranium tuberosum L.

Graminées

Agrostis verticillata Vill.

Arundo Donax L.

Avena barbata Brot.

A. sterilis $\mathrm{L}$.

Bormus macrostachys Desf.

B. rubens $\mathbf{L}$.

B. scoparius $\mathrm{L}$.

Erianthus Ravennae (L.) Beauv.

Hordeum bulbosum L.

Phalaris minor Retz.

Iridacées

Gladiolus segetum Gawl.

Iris Sisyrinchium $\mathbf{L}$.

Labiées

Salvia viridis $\mathbf{L}$.

Légumineuses

Coronilla scorpioides (L.) Koch

Hymenocarpus circinnatus (L.) Savi Lathyrus annuus L. 
Medicago coronata Desr.

Trifolium lappaceum $\mathrm{L}$.

Vicia peregrina $L$.

Iiliacées

Allium Ampeloprasum L.

A. paniculatum $\mathbf{L}$.

Gagea reticulata (Pall.) Sch.

Malvacées

Malva nicaensis All.

M. parviflora $L$.

Malvella Sherardiana (L.) J. et Sp.

Ombellifères

Ammi Visnaga (L.) Lam.

Caucalis leptophylla $L$.

Daucus maximus Desf.

Lagoecia cuminoides L.

Papaveracées

Roemeria hybrida (L.) DC.

Plantaginacées

Plantago Lagopus L.
Polygonacées

Polygonum equisetiforme Sibth. et Sm.

Polypodiacées

Cheilanthes fragrans (L.) Webb et Berth.

Primulacées

Asterolinum Linum-stellatum (L.) LK. et Hoffm.

Renonculacées

Anemone coronaria $\mathrm{L}$.

Delphinium peregrinum L.

Ranunculus muricatus $L$.

R. trachycarpus $\mathbf{F}$. et $\mathbf{M}$.

Scrofulariacées

Verbascum sinuatum $L$.

Veronica cymbalaria Bod.

Urticacées

Parietaria lusitanica $\mathrm{L}$.

Verbenacées

Vitex Agnus-Castus L.

\section{b. Espèces sub-méditerranéo-irano-touraniennes}

\section{Borraginackes}

Anchusa italica Retz.

Echium italicum L.

Heliotropinm europaeum L.

H. supinum $L$.

Oaryophyllacées

Arenaria leptoclados Rchb.

Cerastium dichotomum $\mathrm{L}$.

Sagina apetala Ard.

Saponaria Vaccaria L.

Chenopodiacées

Chenopodium vulvaria $L$.

Atriplex rosea L.

Composées

Calendula arvensis $L$.

Crepis foetida $\mathrm{L}$.

Helminthia echioides (L.) Gaertn.

Lactuca saligna $\mathrm{L}$.

Cruciferes

Alyssum campestre L.

Conringia orientalis (L.) Andr.

Erophila praecox (Stev.) DC.

Lepidium Draba L.

Neslia paniculata (L.) Desr.

Rapistrum rugosum (L.) All.

Sisymbrium Irio $L$.
Cypéracées

Cyperus glaber $\mathbf{L}$.

C. longus $L$.

Seirpus Holoschoenus L.

Fumariacées

Fumaria parviflora Lam.

Géraniacées

Erodium moschatum (L.) L'Hér.

Graminées

Brachypodium distachyum (L.) $\mathbf{R}$. et $\mathbf{S}$.

B. pinnatum (L.) P. B.

Bromus madritensis L.

B. sterilis L.

Crypsis aculeata (L.) Ait.

Heleochloa schoenoides (L.) Host.

Hordeum maritimum With.

Koeleria phleoides Pers.

Lamarckia aurea (L.) Moench

Lepturus incurvatus (L.) Trin.

Lolium rigidum Gaud.

Phalaris paradoxa $L$.

Polypogon maritimum Willd.

Vulpia ciliata (Pers.) Link.

Juncacées

Juncus acutus L. 


\section{Labiées}

Marrubium vulgare L.

Légumineuses

Astragalus hamosus L.

Lathyrus aphaca $\mathbf{L}$.

Lotus angustissimus $\mathbf{L}$.

Medicago minima Lam.

M. orbicularis All.

Scorpiurus sulcata L.

Trifolium resupinatum $\mathrm{L}$.

T. scabrum $\mathbf{L}$.

Trigonella monspeliaca $L$.

Vicia sativa $L$.

\section{Liliacées}

Muscari comosum Mill.

M. racemosum (L.) Lam. et DC.

Ornithogalum umbellatum $L$.

\section{Linacées}

Linum strictum L.

Ombellifères

Ammi majus L.

Bupleurum lancifolium Hornem

Seandix Pecten-Veneris L.

Torilis infesta (L.) Hoffm.

T. neglecta $R$. et Sch.

T. nodosa (L.) Gaertn.

\author{
Orehidacées \\ Orchis laxiflorus Lam. \\ Papaveracées \\ Glaucium corniculatum (L.) Curt. \\ Polygonacées \\ Rumex pulcher L. \\ Polypodiacées \\ Ceterach officinarum Willd. \\ Renonculacées \\ Nigella arvensis $\mathbf{L}$. \\ Résédacées \\ Reseda lutea L. \\ R. luteola $\mathrm{L}$. \\ Rubiacées \\ Galium tricorne Stokes. \\ Sherardia arvensis $\mathrm{L}$. \\ Serofulariacées \\ Veronica anagalloides Guss. \\ Urticacées \\ Parietaria judica $L$. \\ Urtica pilulifera $L$. \\ Valerianacées \\ Valerianella coronata (Willd.) DC. \\ V. truncata (Rehb.) Betcke \\ Zygophyllacées \\ Tribulus terrestris L.
}

\section{c. Espèces méditerranéo-orientales-irano-touraniennes}

\section{Asclépiadacées}

Periploca graeca L.

Berbéridacées

Bongardia chrysogonum (L.) Boiss.

Borraginacées

Lithospermum tenuiflorum $L$. fil.

Podonosma syriacum (Lab.) Boiss.

Campanulacées

Campanula strigosa Russ.

Caryophyllacées

Silene chretodonta Boiss.

Composées

Anthemis pseudocotula Boiss.

Centaurea Behen L.

Serratula cerinthefolia Sibth. et Sm.

Tragopogon longirostre Bisch.

Convolvulacées

Cuscuta palaestina Boiss.

Orassulacées

Umbilicus intermedius Boiss.
Cueurbitacées

Bryonia multiflora Boiss. et Heldr.

Dipsackes

Cephalaria syriaca (L.) Schr.

Euphorbiacées

Euphorbia aleppica L.

Labiées

Zizyphora capitata L.

Liliacées

Bellevalia macrobotrys Boiss.

Ornithogalum montanum Cyrill.

Malvacées

Althaea lavateraeflora DC.

Ombellifères

Artedia squamata L.

Bupleurum odontites L.

Caucalis tenella Del.

Coriandrum sativum $L$.

Scandix iberica M. B. 
Primulacées

Lysimachia dubia Qit.

Renonculacées

Ranunculus asiaticus $L$.

R. hierosolymitanus Boiss.

R. lomatocarpus F. et M.

Rutacées

Haplophyllum Buxbaumi (Poir.)

Boiss.
Scrofulariacées

Celsia orientalis $\mathrm{L}$.

Tamaricacées

Tamarix Pallasii Desv.

Valerianacées

Valerianella vesicaria

Moench

(Willd.)

2. Plantes de liaison eurosiberiennes-boreo-americaines-méditerranéo-irano-touraniennes (Liaison Eurosib.-Boreoam-Medit.-Ir. Tour.)

Parmi les espèces de ce groupe nous citerons :

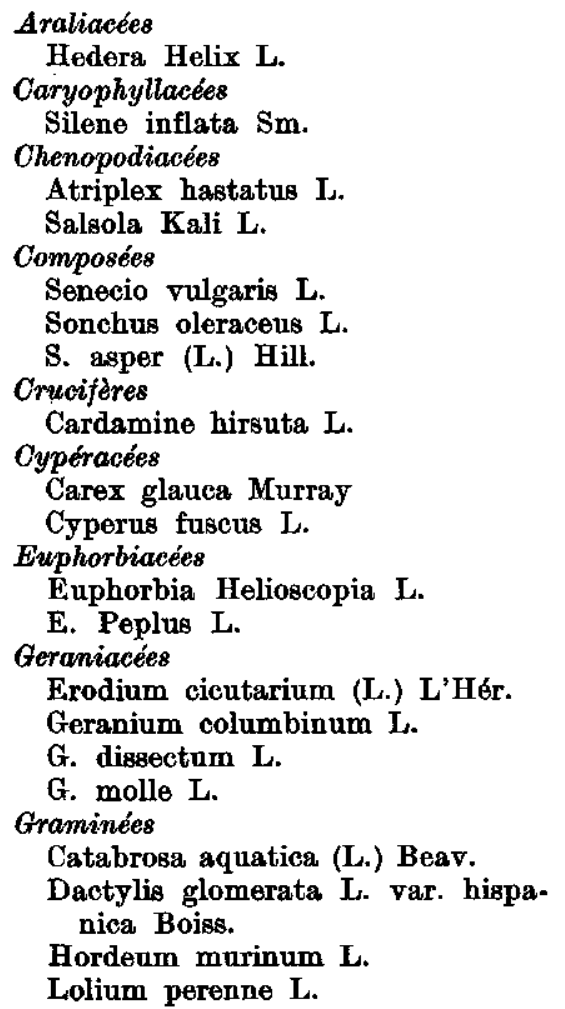

L. temulentum L.

Poa annua $\mathbf{L}$.

P. bulbosa $\mathrm{L}$.

Iridacées

Iris pseudoacorus $\mathrm{L}$.

Légumineuses

Lotus tenuifolius (L.) Rehb.

Medicago lupulina $\mathbf{L}$.

Trifolium fragiferum $\mathrm{L}$.

Malvacées

Malva silvestris $\mathbf{L}$.

Nymphéacées

Nuphar luteum (L.) Sm.

Nymphaea alba $\mathrm{L}$.

Oenotheracées

Epilobium tetragonum L.

Orchidacées

Cephalanthera longifolia Fritseh

Epipactis latifolia All.

Plantaginacées

Plantago lanceolata $\mathrm{L}$.

P. major L.

Polygonacées

Rumex crispus $\mathbf{L}$.

Renonculacées

Ranunculus arvensis L.

Rosacées

Agrimonia Eupatoria L.

Salicacées

Salix alba $\mathbf{L}$. 
Un certain nombre de ces plantes de liaison se place dans le catégorie des plantes rudérales et ségétales. La présence d'une dizaine d'espèces de ce groupe (Cypéracées, Juncacées, Graminées) est liée aux habitats humides et a généralement une valeur moindre, au point de vue phytogéographique, que les plantes ségétales et rudérales.

En déduisant les plantes rudérales, ségétales et hydrophiles, il restera les plantes qui ont une valeur plus grande au point de vue des affinités phytogéographiques. Elles nous permettent de juger la parenté de notre Région avec la Région Irano-touranienne voisine. Les versants humides des montagnes de la Perse paraissent avoir une série d'enclaves méditerranéennes, par ex. : le Kurdistan, le Karah-Dagh, etc. C'est dans ces enclaves montagneuses que croissent les nombreuses espèces subméditerranéennes ayant une forte pénétration Irano-touranienne. D'après Eig, c'est ici que se trouve concentrée une grande quantité de plantes de liaison méditerranéo-irano-touraniennes. Un nombre beaucoup moins élevé de ces plantes lie notre Région avec la Région eurosibérienne-boréo-américaine.

\section{V - LES PLANTES PLURIREGIONALES OU COSMOPOLITES}

D'après leur répartition géographique, ces plantes peuvent être divisées en deux groupes:

\section{a. Espèces boréo-tropicales}

\author{
Amarantacées \\ Amaranthus Blitum Kth. \\ A. angustifolius Lam. \\ A. retroflexus $\mathrm{L}$. \\ Caryophyllacées \\ Spergula arvensis $\mathrm{L}$. \\ Spergularia marginata Kittel \\ Stellaria media (L.) Cyrill. \\ Chénopodiacées \\ Chenopodium album $\mathrm{L}$. \\ C. ambrosioides $\mathbf{L}$. \\ C. murale $\mathrm{L}$.
}

Salicornia fruticosa L.

$$
\begin{aligned}
& \text { Suaeda fruticosa (L.) Forsk. } \\
& \text { Composées } \\
& \text { Gnaphalium luteo-album L. } \\
& \text { Xanthium spinosum L. } \\
& \text { Convolvulacées } \\
& \text { Convolvulus arvensis L. } \\
& \text { C. sepium L. } \\
& \text { Crueiferes } \\
& \text { Capsella bursa pastoris (L.) Moench } \\
& \text { C. procumbens Fries } \\
& \text { Nasturtium officinale R. Br. }
\end{aligned}
$$




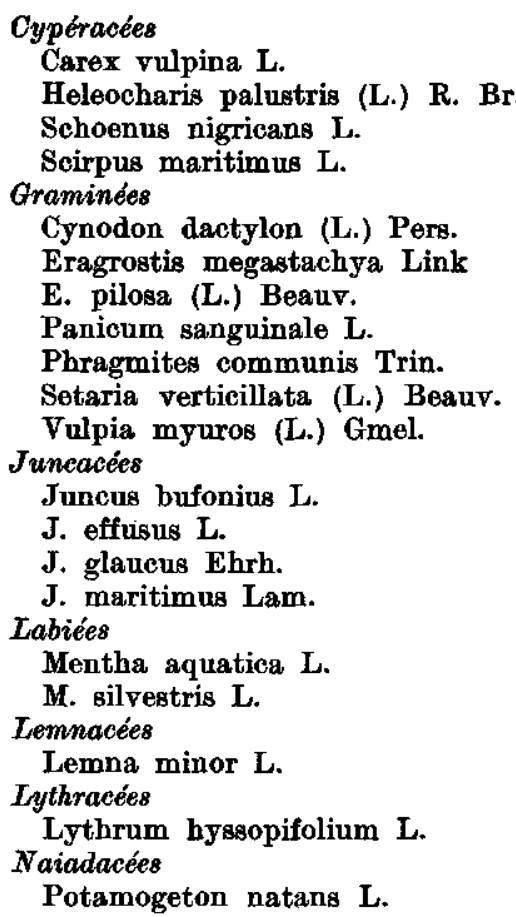

Ruppia spiralis (L.) Dum.

Zannichellia palustris $\mathbf{L}$.

Ombellifères

Apium graveolens $\mathbf{L}$.

Helosciadium nodiflorum (L.) Koch

Orobanchacées

Orobanche ramosa L.

Polygonacées

Polygonum aviculare $\mathbf{L}$.

P. lapathifolium $\mathrm{L}$.

Polypodiacées

Asplenium Adiantum-nigrum $L$.

Polypodium vulgare $L$.

Pteridium aquilinum (L.) Kuhn

Portulacacées

Portulaca oleracea $L$.

Primulacées

Anagallis arvensis L.

Samolus Valerandi L.

Solanacées

Datura Stramonium L.

Solanum nigrum $L$.

Urticacées

Urtica urens L.

Verbenacées

Verbena officinalis L.

La presque totalité de ces plantes et auxquelles on donne en général le nom de "cosmopolites", croit dans des stations humides, rudérales et ségétales. Quelques unes d'entre elles sont halophiles.

\section{b. Espèces subtropico-tropicales}

\section{- Espèces méditerranéo-tropicales}

Andropogon distachyus $L$.

Hermarthria fasciculata (Desf.) Polygonum serrulatum Lag. Hel. Kunth

Smilax aspera $\mathbf{L}$.

- Espèces méditerranéo-irano-tourano-tropicales

Cyperus globosus All.

C. rotundus $\mathbf{L}$.

Frankenia pulverulenta L.

F. hirsuta $L$.

Gymnogramma leptophylla

Desv.
Glinus lotoides I.

Hibiscus trionum L.

Panicum colonum L.

Scirpus littoralis Schrad.

(L.) Withania somnifera (L.) Dun. Ch.

\section{- Espèces méditerranéo-orientales-irano-tourano-tropicales}

Cyperus pygmaeus Rottb.

Typha angustata Bory ot Chaub. 
- Espèces méditerranéo-irano-tourano-saharo-sindiennes-tropicales

Adiantum Capillus-Veneris $\mathbf{L}$.

Andropogon hirtus L.
Cyperus laevigatus $L$.

Imperata cylindrica (L.) Beauv.

Ces plantes sont répandues dans le N.W. de la Syrie sur des stations bien spéciales. Certaines comme: Glinus lotoides, Typha angustifolia, Cyperus rotundus, C. globosus, C. laevigatus se rencontrent dans les fossés et les lieux humides du Littoral; Gymnogramma leptophylla Desv. se rencontre dans les grottes et sur les murs et talus humides et ombragés ; Adiantum Capillus-Veneris L., sur les bords des canaux, rochers et murs humides. Les exigences édaphiques d'un grand nombre de ces plantes explique leur dispersion large. Certaines d'entre elles sont rudérales et ségétales.

Une mention spéciale doit être faite pour Smilax aspera qu'on trouve répandu dans la garrigue à Quercus calliprinos et la forêt de Pinus brutia dans le Cassius et le Djebel Alaouite et dans les garrigues à Quercus coceifera et les taillis à Quercus Ilex dans le Midi de la France. Cette plante est assez répandue aussi aux Indes, depuis l'Himalaya jusqu'à Ceylan. On la trouve également en Abyssinie (EıG, 1933). Il est difficile de soupçonner cette répartition aussi large dans ces régions naturelles aussi variées.

\section{Vi - Les Plantes endemiques}

Du point de vue phytogéographique et floristique le BaerBassit et le Djebel Alaouite n'ont pas un caractère propre. En effet, les massifs du Baer-Bassit sont les prolongements sud en territoire syrien des chainons tures de l'Amanus; le Djebel Alaouite peut être rattaché au Liban Nord, qui est situé plus au Sud. Le Djebel Akra ( ${ }^{1}$ ) (Mt Cassius) peut être rattaché aussi à l'Amanus. C'est ainsi que nous trouvons une affinité floristique assez nette entre le Baer-Bassit et les régions montagneuses inférieures de l'Amanus, d'une part, et le Djebel Alaouite et le Liban Nord d'autre part. Cette affinité existe même dans la végétation. Il est toutefois utile de signaler que l'Amanus qui est situé plus au Nord et possédant des altitudes plus élevées renferme des

(1) Quand les botanistes parlent de la flore du Cassius, ils entendent par ce terme, la flore du Mont Cassius et du Baer-Bassit ensemble. 
espèces nordiques ou septentrionales beaucoup plus que le Baer et le Bassit. Certaines d'entre elles trouvent leur limite Sud dans l'Amanus et ne pénètrent pas en Syrie, comme Fagus silvatica var. orientalis, Ilex aquifolium, Tilia argentea, Taxus baccata, Alnus incana, Asplenium septentrionale, Fragaria vesca, Atropa Belladonna, Asperula odorata, Lathraea squamaria, Sanicula europaea.

Au point de vue géologique, la Montagne Alaouite est semblable au Liban. Il est donc normal qu'à altitudes égales, la flore soit à peu près la même dans les deux massifs. Toutefois, dans sa partie septentrionale, le Djebel Alaouite est très boisé et se raccorde presque sans interruption aux forêts du Baer-Bassit. Cette circonstance, qui crée entre les deux contrées voisines des analogies de climat et d'habitat, explique peut être qu'au delà d'une ligne reliant Banias à Quadmous, on peut trouver dans la région située au Nord de cette ligne des espèces que l'on rencontre plus au Nord dans le Baer et le Bassit, mais qu'on ne saurait trouver au deça de cette limite, par ex. : Triadenia Russegeri Fenz., Nigella stellaris Boiss. (Gombault, 1945).

Nous ne serons donc pas surpris de ne pas trouver beaucoup d'espèces endémiques dans le Baer-Bassit et le Djebel Alaouite.

Les espèces suivantes peuvent être classées provisoirement comme endémiques dans le Baer-Bassit et le Djebel Alaouite:

Astragalus eriophylloides Rech. fil. trouvé à Slenfé, dans la montagne des Alaouites et récolté par HaradjIan (Reliquiae Samuelsonianae VI. Stockholm, Arkiv för Botanik II, V, I, 1959).

Astragalus nusairiensis Eig et Sam. : récolté au col d'Ain el Beida dans la montagne des Alaouites par HARAdJIaN ; même référence que pour l'espèce précédente.

Peucedanum mucronatum Thiébaut: d'après P. MotTerde la présence de cette espèce est presque süre en territoire turc, PABot l'ayant trouvée tout près de la frontière turque, vers Chakaourane.

Scrophularia nusairiensis Post : cette espèce a été trouvée aux environs de Banias, sur le littoral syrien.

Sideritis nusairiensis Post : espèce citée comme endémique dans le Djebel Alaouite par Gombault (Aperȩu sur la flore de la Syrie et du Liban et de la région d'Antioche, 1945).

Allium calyptratum Boiss. Anthemis cassia Boiss. Pyrethrum cassium Boiss.

Trigonella cassia Boiss. Vicia cassia Boiss. citées comme endémiques dans le Cassius (par Gombault, 1945)

considérées comme endémiques jusqu'à nouvel ordre par P. Mouterde (1961). 


\section{VI - ESSAIS SUR LE DEVELOPPEMENT DE LA FLORE DU BAER-BAS- SIT ET DU DJEBEL ALAOUTHE}

\section{Paléoclimatologie et próhistoire}

Les travaux entrepris en Syrie et au Liban pour les études préhistoriques et paléoclimatiques sont à leur début et ne suffisent pas à eux seuls pour tenter l'esquisse de la Préhistoire dans ces deux pays. Les recherches entreprises en Palestine par BaTE, Picard, Stekelis, Vaufrey et d'autres, peuvent être d'une grande utilité pour la compréhension des changements climatiques survenus au Quaternaire en Syrie et au Liban et leur influence sur la faune et la flore ainsi que sur l'homme.

Sans s'accorder toujours dans les détails, les observations de ces auteurs peuvent fournir un schéma pouvant servir de base à notre étude.

Ces auteurs ont reconnu en Palestine, au cours du Pleistocène, deux périodes pluviales principales, séparées par un Interpluvial.

Ils sont également d'accord pour mettre en relation le deuxième Pluvial (Pluvial $B$ ) avec la dernière glaciation dite Würmienne. La période plus sèche, précédente représenterait ainsi la fin de l'interglaciaire Riss-Würm. Ils se montrent plus hésitants quant anx concordances du premièr Pluvial (Pluvial A) avec les glaciations de Riss ou Mindel.

Certains ont cru reconnaître l'existence d'un Pluvial $\mathrm{C}$ de faible importance, se terminant aux environs de l'âge du Bronze.

Pendant les Pluviaux, le climat était très pluvieux et humide et s'est manifesté par d'importants alluvionnements. Le Pluvial A de Picard est matérialisé par d'importants dépôts de graviers; on $y$ a trouvé du Chelléen, de l'Acheuléen inférieur ainsi que des ossements d'éléphants. Le Paléolithique ancien final et le Paléolithique moyen sont richement représentés dans certaines cavernes (Oum Qatofa, Arhlit, etc.) par une succession de niveaux de l'Acheuléen moyen et supérieur, du Micoquien, du Levalloiso-Moustérien et du Moustérien. Ces industries sont accompagnées d'une faune d'aspect africain: Rhinocéros, Hippopotames, Eléphants, Phacochères et microfaune à espèces pri- 
mitives éteintes. De nombreux ossements humains néanderthaloïdes $\mathrm{y}$ ont été découverts également.

A partir des niveaux acheuléen supérieur au achenléen inférieur, selon les vues de PICARD, la faune dénote un assèchement progressif du climat jusqu'à l'avènement du Pluvial B. La faune précitée disparât ; quelques espèces nouvelles, l'Hyène tachetée, le loup, etc. la remplacent.

Ver la fin de cette époque s'implante une faune nettement eurasiatique moderne : Ours brun, Cerf, Gazelle, Marte, Blaireau, Chat sauvage et Dain en grand nombre; les anciennes industries font place à l'outillage sur lames. Les ossements humains, peu nombreux rencontrés dans les couches caractéristiques, appartiennent, à un Homo sapiens à caractères primitifs (J. HALLAIRE, 1945).

Le Pluvial B se termine par une période aride ; le Paléolithique supérieur est remplacé par différents faciès du Mésolithique. Son représentant le plus remarquable, le Natoufien, par ses objects d'art, ses faucilles, etc. dénote un degré de civilisation avancé. Ses auteurs sont de la race méditerranéenne.

A partir de cette époque apparaissent les dernières industries de l'âge de la pierre: Tahounien, Ghassoulien, Cananéen. Ces outillages néolithiques et énéolithiques, qui se présentent à la base de plusieurs tells, passent graduellement aux civilisations protohistoriques et historiques.

Les recherches faites sur le Quaternaire côtier de Tripoli (Liban) par René WETzEL et J. HALLEN ont montré que la nappe alluviale du niveau de $45 \mathrm{~m}$ semble bien répondre au Pluvial principal (Pluvial A) qui a été mis en évidence en Palestine. Les alluvions postérieures au nivean de $6 \mathrm{~m}$ correspondent, d'après les vestiges archéologiques, au petit Pluvial (Pluvial B) de Palestine. L'intervalle entre ces deux époques pluviales est caractérisé par la grande extension des dunes, indiquant ainsi un climat sec. Les recherches ont montré également que les premières manifestations humaines observées précèdent immédiatement la première époque pluviale (Pluvial A). Les niveaux entre 3 et $4 \mathrm{~m}$ et la surface montrent un climat plus sec que le Pluvial (B) et qui correspond au climat actuel.

Ce qui précède nous montre que les données sur le climat du- 
rant la période préhistorique reste encore vague en Syrie et au Liban; mais les connaissances déjà acquises nous permettent de résumer les changements climatiques qui ont pu survenir pendant cette période et montrer leur influence sur la composition de la flore du Baer-Bassit et du Djebel Alaouite :

1) Au Pliocène supérieur commence la première période pluviale (Pluvial A). Le climat est très humide et pluvieux et s'est manifesté par d'importants alluvionnements.

2) Une période sèche (Interpluvial) succède au Pluvial A. Le climat est sec et plus chaud qu'aujourd'hui. Cette période correspondrait à la fin de l'interglaciaire Riss-Würm.

3) Une deuxième période pluviale (Pluvial B), plus courte que la première, vient ensuite et correspond à la dernière glaciation dite Würmienne. Le climat est pluvieux et humide et s'est manifesté par des alluvionnements importants.

4) Le pluvial B se termine par une période aride et le climat ressemblerait au climat actuel.

5) Vient ensuite une troisième période pluviale de très courte durée et beaucoup plus faible que les précédentes. Le climat est humide.

6) Enfin s'établit le climat actuel.

Beaucoup d'auteurs (BIANCKENHoRN, J. HuXLEY, etc.) affirment que depuis les temps historiques on ne constate aucun changement climatique dans nos régions. Il est exact que le climat a subi des modifications considérables pendant la Préhistoire, depuis le dernier recul des glaciers, vers 12.000 ans avant JésusChrist, jusqu'au début de l'Agriculture, aux alentours de l'an 6.000 avant notre ère. Toutefois, depuis la période historique et sans doute depuis 5.000 ans avant Jésus Christ environ, on ne trouve aucune trace de variations climatiques de grandes amplitudes, qu'elles soient soudaines ou progressives, ni même des fluctuations très marquées. Quelques modifications légères et ne portant que sur quelques centaines d'années se sont seules produites, tel le réchauffement des latitudes Nord du premier millénaire avant l'ère chrétienne et la période plus froide qui a commencé vers 1.300 de notre ère et est actuellemént en voie de regression. 


\section{Développement de la flore du Baer-Bassit et du Djebel Alao uite}

\subsection{LA FLORE AU TERTIAIRE}

Le Baer-Bassit et le Djebel Alaouite sont liés aux régions avoisinantes (Amanus, Liban, etc.) au point de vue développement de leur flore.

Si on compare la flore de l'Ile de Chypre avec celle du N.W. de la Syrie, de l'Amanus et du Liban et la flore de la Corse avec celle du Midi de la France et de l'Italie, nous constaterons qu'il y a une grande analogie floristique entre Chypre et ses pays voisins dont la flore est essentiellement Est-méditerranéenne et la Corse et ses pays voisins dont la flore est Ouest-méditerranéenne. Braun-Blanquet (1924) démontre clairement que l'analyse de la flore de la Corse nous oblige d'admettre que cette ile s'était séparée du continent au Miocène supérieur ou au Pliocène inférieur.

Le même raisonnement peut être fait pour l'Ile de Chypre qui, d'après les géologues, était déjà complètement séparée du continent avant la Pliocène.

La flore méditerranéenne présentait donc déjà à la fin du Tertiaire ses traits les plus importants et en particulier ses subdivisions en flores Est et Ouest méditerranéennes.

Nous pouvons également supposer, malgré l'absence de données précises sur place, que déjà au Pliocène l'élément méditerranéen présentait en Syrie, Liban et Palestine ses traits les plus importants. Ce qui rend encore plus plausible cette hypothèse c'est le fait que, d'après BLANCKENHORN (1910) le climat du Pliocène ne devait pas être très différent du climat actuel.

\subsection{LA FLORE AU QUATERNAIRE}

L'étude paléoclimatique nous a montré que la Syrie, le Liban et la Palestine (les régions du Baer-Bassit et du Djebel Alaouite incluses) ont connu au Quaternaire une période de pluviosité intense ayant coincidé avec la période glaciaire en Europe. Ces modifications pluviales survenues au Quaternaire, ont laissé une 
empreinte très visible de nos jours et sans elles beaucoup de problèmes phytogéographiques ne sauraient être expliqués.

C'est ainsi que nous pouvons expliquer le cantonnement de certaines espèces nordiques ou septentrionales dans les parties les plus élevées de la Montagne des Alaouites, constituant ainsi des enclaves septentrionales parmi une flore typiquement méditerranéenne. Parmi ces plantes septentrionales nous avions cité (voir enclaves nordiques): Sorbus torminalis, Sorbus umbellata, Cerasus Mahaleb, Daphne oleoides, Corylus avellana, Staphylea pinnata, Inula conyza, ete.

On peut expliquer de la même façon le grand nombre d'espèces septentrionales localisées dans l'Amanus et ne pénétrant pas en territoire syrien comme: Fagus silvatica var. orientalis, Ilex aquifolium, Fragaria vesca, Asplenium septentrionale, Atropa Belladonna, Asperula odorata, etc.

En effet, le refroidissement survenu au Tertiaire en Europe et les glaciations Quaternaire ont poussé devant eux, vers l'Est, des plantes d'Europe. Arrivées pendant la période pluviale, ces plantes s'y sont installées.

Arrive ensuite la période sèche caractérisée par un climat plus sec et plus chaud que celui de la période pluviale. Le climat ne leur convenant plus, les plantes dominantes du Pluvial se retirent à leur tour, périssent en masse, ou réussissent à se maintenir sous forme de reliques pluviales sur les hautes altitudes où le climat est plus humide et plus frais.

Il est intéressant de remarquer qu'en Europe, avec la fin de la période glaciaire, s'ouvrent pour la flore de grandes possibilités d'expansion, de développement, d'envahissement de territoires immenses nouveaux. Dans nos régions, comme d'ailleurs dans toutes les régions qui ont connu une période pluviale Quaternaire, c'est juste le contraire. En effet, avec la fin de la période pluviale, période très favorable au développement de la flore dans un pays sec, commence pour notre flore, une période de dégradation relative, de diminution, de pertes en espace et en espèces. Mais, en Europe comme chez nous, Période glaciaire et Période pluviale sont de première importance pour la flore et sa distribution. 


\section{VII - CARACTERES DE LA FLORE DU BAER-BASSIT ET DU DJEBEL ALAOUTE}

Nous avons vu que l'élément méditerranéen et ses sous-groupes constituaient presque la totalité de la flore du N. W. de la Syrie. Cette flore est par conséquent bien adaptée anx conditions climatiques qui règnent dans cette région, et qui sont des conditions de climat méditerranéen. Des étés chauds et secs de longue durée, des hivers courts et tempérés, un éclairement intense, deux périodes annuelles de ralentissement de la végétation, l'une estivale déterminée par la température élevée et la sécheresse accrue en particulier dans la Méditerranée orientale, l'autre hivernale déterminée par le froid, tels sont les caractères essentiels et déterminants du climat méditerranéen oriental.

Les plantes sont adaptées aux conditions spéciales de ce milieu et présentent des formes adaptatives variées; il en résulte que les espèces les mieux adaptées sont les plus favorisées dans la lutte pour la vie, qu'elles tendent a devenir envahissantes et qu'elles sont très habituellement les plus répandues, autrement dit les plus nombreuses en individus... Au niveau de la plante elle-même, ce qui caractérise le plus spécialement le climat de la Méditerranée orientale, c'est le défaut d'équilibre assez prononcé entre la quantité d'eau mise à la disposition de la plante (malgré la forte pluviométrie annuelle qui dépasse $800 \mathrm{~mm}$ par an, une faible partie seulement est mise à la disposition des végétanx, l'autre partie disparaît par ruissellement et par évaporation) et les conditions atmosphériques très favorables à la transpiration. Nos espèces sont par conséquent adaptées à lutter contre l'excès de la transpiration. Elles sont donc essentiellement xérophiles. Certaines d'entre elles sont hygrophiles et possèdent des feuilles assez larges favorisant la transpiration, mais elles se trouvent localisées dans des conditions très spéciales d'altitude, d'exposition favorable et de sol qui leur permettent de subsister. C'est le cas du Quercus pseudocerris du Baer-Bassit qu'on rencontre à partir de $400 \mathrm{~m}$ d'altitude sur des sols profonds emmagasinant l'eau et sur les expositions les plus favorables (Nord et Nord Ouest); il ne s'eloigne pas beaucoup de la côte.

Très peu d'expériences directes ont été faites sur les adapta- 
tions des espèces méditerranéennes. Aussi devons-nous dans ce qui suit nous fonder sur les observations faites dans la nature par beaucoup de botanistes et dans différents pays méditerranéens et sur le fait que le rôle des espèces méditerranéennes c'est d'échapper au défaut d'équilibre entre la quantité d'eau mise à leur disposition et les conditions atmosphériques qui favorisent la transpiration, ou lutter contre lui.

Nous allons montrer dans ce qui suit comment les végétaux spontanés du Baer-Bassit et du Djebel Alaouite arrivent à s'adapter au climat méditerranéo-oriental:

1) Certaines espèces font pendant la saison favorable qui est l'hiver des réserves d'eau qu'elles utilisent pendant la saison sèche. C'est le cas des Sedum, Saxifraga, etc. Cette forme d'adaptation est la moins répandue parmi les espèces de notre région.

2) Certaines réduisent leur surface transpiratoire ; la réduction peut être de deux façons, momentanée ou continue.

a) réduction momentanée de la surface foliaire : les feuilles linéaires d'un certain nombre de graminées xérophiles de notre région sont planes, lorsque l'air et le sol sont suffisamment chargés d'eau et s'enroulent par leurs deux bords de manière à recouvrir les lignes de stomates de leur face supérieure. Cette propriété permet à certaines graminées (les Stipa, Triticum, Hyparrhenia, etc.) de notre région de coloniser les sols les plus dégradés et les plus secs, par exemple les sols très dégradés et très secs provenant de l'évolution régressive du Pistacieto-Quercetum calliprini. Les feuilles de ces plantes sont étalées pendant la saison humide, junciformes pendant la saison sèche.

Dans d'autres cas, la feuille modifie son orientation de manière à offrir aux radiations lumineuses une surface moindre. Tel est le cas de deux espèces très répandues dans le Baer-Bassit et le Djebel Alaouites: le Cercis Siliquastrum (arbre de Judée) et le Vitex Agnus-Castus dont les folioles se rabattent aux heures les plus éclairées, pour reprendre très rapidement leur position première dès que l'éclairement n'est plus excessif.

C'est également le cas du Celtis australis (Micocoulier), espèce méditerranéenne assez répandue dans notre région, non spontanée, mais complètement naturalisée. Le Celtis australis dont les feuilles ont une position rapprochée de la position horizontale dans les 
conditions normales, les incline, la pointe tournée vers le bas lorsque le soleil est au Zenith, en même temps que les deux bords se rabattent plus ou moins vers la face supérieure sur la nervure médiane. Il en résulte qu'aux heures les plus éclairées des jours d'été, le Celtis australis donne très peu d'ombre. Dès que l'intensité de la lumière diminue, les feuilles de Celtis reprennent leur position normale.

Cette réduction momentanée de la surface foliaire est beaucoup moins répandue que la réduction continue. Car les conditions qui tendent à rendre la transpiration excessive étant les plus habituelles sous le climat de notre région et s'y exercent presque partout d'une manière continue la lutte contre l'excès de la transpiration doit être continue aussi. Donc, ce n'est pas par des adaptations transitoires, mais par des modifications continues définitivement acquises, que la plupart des espèces méditerranéennes de notre région arrivent à s'y maintenir et se perpétuer.

Nous allons examiner les différentes formes d'adaptations continues qui se présentent dans notre région:

b) réduction continue de la surface foliaire : les faibles dimensions de la feuille représentent le moyen le plus simple par lequel la plante réduit sa surface transpiratoire.

Les feuilles réduites peuvent être en aiguilles comme dans le Pinus brutia, espèce adaptée aux conditions xérophiles et se rencontrant dans le Bayer et le Bassit sur les sols les plus superficiels formés sur serpentines, ne retenant pas l'eau et constituant ainsi un milieu édaphique très sec; elles sont, ailleurs, éricoides plus ou moins enroulées vers la face inférieure comme dans Erica verticillata qui se rencontre dans les groupements de dégradation; ou aplaties en écailles, cupressoides, comme dans le Cupressus sempervirens, Tamarix pentandra, etc.

Iues feuilles réduites peuvent même l'être au point de ne plus suffire comme organes assimilateurs, les feuilles peuvent être éphémères comme chez Calycotome villosa, Genista acanthoclada qui sont répandues dans les groupements de dégradation et sur des sols très secs. Le même phénomène se voit chez Spartium junceum; il faut alors qu'elles soient remplacées partiellement au moins.

Les feuilles réduites au point de n'être plus assimilatrices peuvent être en partie supplées dans leurs fonctions par des ra- 
meaux de forme spéciale, comme les cladodes qu'on trouve chez Ruscus aculeatus et Asparagus sp., ou les épines chez Genista acanthoclada, Calycotome villosa, etc., les rameaux tout entiers peuvent être chargés de la fonction assimilatrice, grâce au développement considérable du tissu assimilateur à la périphérie de leur écorce, comme chez Spartium junceum.

Quelque soit la modification subie par la feuille pour diminuer sa surface transpiratoire et de la tige pour contribuer à la fonction assimilatrice, certaines dispositions anatomiques contribuent encore à protéger la plante contre l'excès de transpiration. Cette forme de protection est très fréquente et assez variée et peut être résumée ainsi :

- Certaines espèces les plus nettement méditerranéennes possèdent des poils protecteurs qui forment à leur surface un feutrage très serré comme chez les Cistus, Ononis, Anthyllis (en particulier chez Cistus villosus qui forme l'élément principal de certains groupements de dégradations sur des sols très secs) et la plupart des Labiées: Thymus, Calamintha, Lavandula stoechas.

- La cutinisation de l'épiderme des feuilles peut être une forme d'adaptation à la sécheresse et se rencontre chez les espèces les plus typiquement méditerranéennes et entrant dans la composition des groupements végétaux les plus caractéristiques de la Méditerranée orientale: Quercus calliprinos avec ses variétés, Phillyrea media, Pistacia Lentisous, Rhamnus Alaternus, Rhamnus palaestina, Rhamnus punctata, Myrtus communis, Lonicera implexa, Acer syriacum, Pistacia palaestina, etc.

- Le Doufla (Nerium oleander), qu'on trouve le long des petits ruisseaux, possède des stomates réunis dans des chambres où ils sont protégés par une couronne de poils. Ce système lui permet de se protéger contre l'excès de la transpiration.

- Des stipules scarieuses abritent les bourgeons et les protègent de la dessication; chez Paronychia ( $P$. argentea Lam. qu'on rencontre dans les lieux arides du littoral ; $P$. capitata Lam., etc.) et Herniaria.

- Enfin, il existe un mode de protection contre l'excès de la transpiration assez particulier chez les Stipa, les Koeleria, les Andropogon. Il est réalisé par la base des feuilles qui persistent longtemps autour des rameaux protégeant ainsi les bourgeons 
naissants sous une sorte de bourre formée par des faisceaux libéroligneux des feuilles anciennes (FLafauts, 1897).

Nous avons vu plus haut comment certaines espèces de notre région méditerranéenne se mettent à l'abri des inconvénients résultant de l'excès de la transpiration en diminuant leur surface transpiratoire dans un grand nombre de cas. Mais l'organe qui transpire est aussi l'organe qui assimile; si l'organe assimilateur a moins de surface, toutes les autres conditions étant égales, il assimilera moins et la plante se trouvera, par cette diminution fonctionnelle, dans une situation d'infériorité, vis-à-vis des espèces qui ne sont pas défendues ou protégées contre l'excès de la transpiration. Les plantes méditerranéennes et en particulier celles de la Méditerranée orientale échappent à cet inconvénient. En effet, l'hiver de notre région est de courte durée et très doux, en particulier sur la côte et dans les basses montagnes. En outre, les heures chaudes et éclairées ne sont pas rares en hiver. Il est, par conséquent, rare que les plantes de notre région arrêtent leur assimilation d'une façon absolue en hiver. Aussi, la majorité des espèces ont-elles des feuilles persistantes, c'est-à-dire durant au moins une année, devenues vieilles, ce n'est pas à l'automne qu'elles tombent, mais ordinairement au coeur de l'été au moment où la température très élevéo les rend plus nuisibles qu'utiles à la plante.

3) Certaines plantes possèdent une couche cireuse qui tapisse les organes assimilateurs; d'autres possèdent des huilles essentielles. Les plantes aromatiques, secrétant des huiles ou des essences, sont très importantes dans la région qui nous occupe. Les ombellifères, les composées, mais surtout de nombreuses labiées, les Cistes, les Anacardiacées sont tout à fait caractéristiques.

4) Enfin, certaines espèces échappent aux conditions défavorables comme les plantes annuelles dont le cycle évolutif s'accomplit tout entier entre les saisons défavorables ou comme les plantes bulbeuses, à organes de réserves souterrains, à organes assimilateurs temporaires, ou pourvus de tubercules ou de rhizomes également à organes assimilateurs temporaires.

Beaucoup d'espèces annuelles germent à l'automne, fleurissent dès les premiers beaux jours du printemps et achèvent leur évolution avant la saison sèche. La graine est un bourgeon dormant 
pourvu de réserves nutritives permettant ainsi à ces plantes annuelles d'éviter la saison défavorable sèche. La plante annuelle est, par conséquent, la forme adaptationnelle la plus simple aux climats temporairement défavorables comme c'est le cas du climat méditerranéen oriental.

Le nombre des espèces annuelles est si grand dans notre région qu'il est inutile de citer des exemples.

Les espèces bulbeuses sont également très nombreuses dans notre région. Nous citons pour exemple : Ornithogalum pyrenaicum, Muscari maritimum, Muscari comosum, Hyacinthus orientalis, Urginea maritima, Colchicum Kotschyi, Fritillaria acmopetala. Les familles des Renoneulacées, Ombellifères, Composées, Liliacées, Orchidées, Iridacées en comptent un nombre considérable. La période de vie latente commence pour la plupart de ces plantes à la fin du printemps et dure jusqu'après les premières pluies d'automne. Dès que les températures moyennes se sont adoucies et que le sol a été humecté par les pluies d'automne après un été long et très sec, des racines commencent à se développer et une rosette de feuilles apparait à la surface du sol grâce à laquelle l'assimilation se fera régulièrement pendant l'hiver.

5) Certaines espèces méditerranéennes de notre région, exigeantes en humidité comme Cedrus Libani, Abies cilicica, Quercus pseudocerris, Quercus Cerris, ainsi que certaines espèces nordiques comme : Sorbus torminalis, Sorbus umbellata, arrivent à se maintenir sous le climat méditerranéen oriental en choisissant des stations favorables, fraïches et humides. C'est ainsi que la majorité de ces espèces se localise en haute altitude où les conditions pluviométriques et hygrométriques leur sont plus favorables.

Le sapin de Cilicie se trouve localisé dans la partie supérieure du Djebel Alaouite et uniquement sur les versants humides et frais, c'est-à-dire, sur les versants ouest, nord et nord-ouest. Le Quercus Cerris ne constitue de belles forêts qu'à partir de 900 à $1000 \mathrm{~m}$ d'altitude. Le Quercus pseudocerris choisit dans le Baer et le Bassit les sols les plus profonds et les expositions fraïches.

6) A la sécheresse accentuée et aux températures élévées estivales s'ajoutent des conditions édaphiques défavorables dans les massifs calcaires, augmentant ainsi la xérophilie du milieu pour les végétaux de notre région. Les sols formés sur calcaire 
cristallin dur et en particulier, les sols dégradés, sont extrêmement secs pendant l'été. Mais, ce qui est heureux, c'est que cess calcaires sont fissurés très profondément et de la terre fine rouge (Terra rossa) s'accumule dans leurs fissures. Les végétanx qui les colonisent y sont adaptés et possèdent la faculté d'émettre deux sortes de racines : 1) de très longues racines qui vont, pendant la saison sèche, à la recherche de l'eau emmagasinée dans la terre fine de ces fissures. Dans ces dernières les racines sont, en outre, à l'abri des hautes températures et de la dessication ; 2) des racines superficielles assez longues, se développant horizontalement, utilisant ainsi l'eau de la partie superficielle du sol. C'est ainsi que l'on a observé des racines allant jusqu'à $5 \mathrm{~m}$ de profondeur chez certaines espèces (Quercus calliprinos, Ceratonia siliqua, Olea europaea) poussant sur calcaire dur fissuré.

Signalons que les racines de certaines espèces sont capables de pénétrer dans des roches plus ou moins tendres (OPPENHEIMER 1956). C'est le cas de: Ceratonia siliqua, Arbutus andrachne, Pistacia palaestina, Pistacia lentiscus, Quercus calliprinos, Rhamnus palaestina, etc., espèces entrant dans la composition des associations principales du Baer-Bassit et du Djebel Alaouite.

\section{Chapitre quatritime}

ETUDE DES TROIS ESPECES FORESTIERES PRINCIPALES (REPARTITION GEOGRAPHIQUE, ETUDE BOTANIQUE, AUTOECOLOGIQUE ET FORESTIERE)

\section{I — PINUS BRUTIA Ten. (1811) \\ Nom loal arabe: Sanaoubar}

1. Aire geographique du Pinus brutia Ten.

Le Pinus brutia est un arbre bien caractéristique de la Méditerranée orientale. Il occupe dans cette région des surfaces très importantes et $y$ constitue de très belles forêts. Son aire géogra- 
phique actuelle est fragmentée. En effet, on le trouve en Grèce (Thrace, Crète, Rhodes, etc.), en Turquie (côtes de la Mer Noire, la mer Marmora, la mer Egée, la mer Méditerranée, Taurus, Amanus), en Syrie (Baer-Bassit, Cassius, Djebel Alaouite, Djebel Akraad), au Liban et à Chypre. On trouve également des petits blocs de $P$. brutia sur les rives Nord-Est de la Mer Noire (Crimée, Pitzunda), au Nord-Est de l'Irak, dans la Perse du Nord et en Afghanistan (cité par BoIssiER).

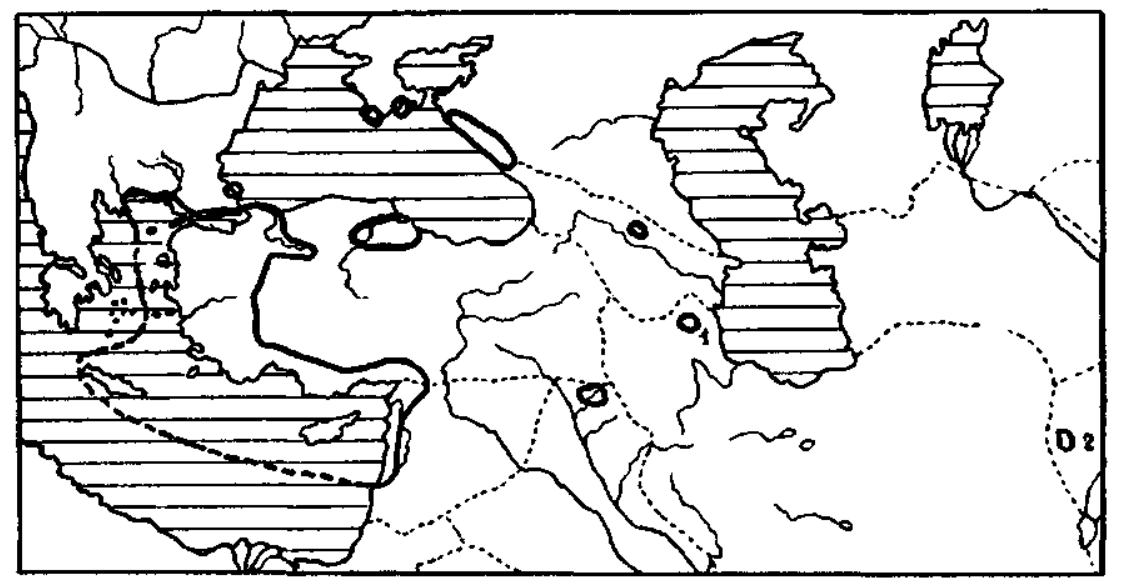

Fig. 5 - Aire géographique du Pinus brutia Ten. (D'après Paratoannod)

Les rives Nord-Est de la Mer Noire constituent la limite la plus septentrionale de l'aire de Pinus brutia, le Sud du Liban constitue la limite la plus méridionale et l'Afghanistan occidental représente la limite la plus asiatique de cette aire.

Le bloc le plus important est celui de Grèce, Turquie, Chypre, Syrie et Liban. C'est dans ces régions qu'il constitue une aire presque continue. Les petits blocs isolés de Crimée, Pitzunda, Iran, Irak, Afghanistan, représenteraient des reliques d'une aire géographique qui était autrefois beaucoup plus importante et plus vaste qu'aujourd'hui.

En Syrie, le Pinus brutia occupe de vastes étendues et constitue les plus belles forêts de notre pays. On le trouve localisé surtout 
dans les régions côtières, dans le Cassius, le Baer-Bassit et le Djebel Alaouite. A l'intérieur, on le voit réapparaitre dans le Djebel Akraad (Kurd Dagh), aux environs d'Alep où il constitue des forêts de second ordre.

D'après J. C. Papajoannou (1954), le Pinus brutia Ten. et le Pinus halepensis Mill. ont une répartition géographique tout à fait séparée et les lignes de démarcation se trouvent nettement limitées en Grèce et dans le Liban sud. Le Pinus halepensis s'étend sur les rives méditerranéennes de l'Afrique du Nord et en Europe, exception faite pour l'Egypte et la Tripolitaine. En Asie, il se réduit seulement à la Palestine et au Liban sud et e'est à partir de Sidon (saïda) et peut être de Beyrouth qu'apparaît le Pinus brutia et se répand plus au Nord au Liban, à Chypre, en Syrie, en Turquie d'Europe et en Grèce. Ce qui revient donc à dire qu'à l'intérieur de l'aire du Pinus brutia, le Pinus halepensis est exclus.

Ceci est vrai, dans l'ensemble, mais les recherches effectuées en Turquie par Dr. HAYRETTIN KAYACIK et par nous-même dans le Nord-Ouest de la Syrie, ont montré que des îlots de Pinus halepensis existent à l'intérieur de l'aire du Pinus brutia, soit à l'état pur soit en mélange avec le Pinus brutia. Dr. KAYACIK a trouvé un ilot de $P$. halepensis en 1952 recouvrant une surface de $16 \times 30 \mathrm{~km}$ dans la forêt de Sariçam, dans le Taurus, à $32 \mathrm{~km}$ de la côte méditerranéenne. Le $P$. halepensis est mélangé au $P$. brutia et pousse sur terra rossa.

En Syrie, nous avons également trouvé un petit îlot de $P$. halepensis dans la région de Qadmous, à gauche de la route BaniasQadmous. Il est accompagné de Pistacia palaestina, Phillyrea media, Juniperus oxycedrus, etc. D'après ALLEGRI, ce pin serait la Pinus pithyusa Stev. En réalité le Pinus pithyusa Stev. n'est que le Pinus brutia Ten. lui-même et le pin de Qadmous diffère beaucoup du Pinus brutia. C'est probablement une forme locale de Pinus halepensis. Les recherches plus approfondies permettront peut être, plus tard, de découvrir d'antres îlots de $P$. halepensis au milieu de l'aire géographique du $P$. brutia. 


\section{Position syst6matique du Pinus brutia}

Le genre Pinus, de la famille des Pinacées (Abietacées), très complexe, est divisé en trois sous-genres et les genres en sections (GaUssen, Les Gymnospermes actuelles et fossiles, 1961).

Ces trois sous-genres sont classés de la façon suivante :

1. Ducampopinus : feuilles par 2, plates, haplostélées, à hydrostéréome transversal. Ce sous-genre ne renferme qu'une section (Krempfioides) et une seule espèce : le Pinus Krempfii Lec. du Vietnam.

2. Eupinus : nombre de feuilles variable, ombilic dorsal, cône ligneux à écailles dures.

Ce sous-genre comprend 5 sections :

- Taedoponderosoides : ex. : P. taeda L. (S.E. de l'Amérique du Nord)

- Merkusioides : ex. : P. Merkusii Jungh et De Vries (Indocine, Ma1aisie)

- Halepensoides : ex. : P. halepensis Mill. (Région méditerranéenne)

- Khasyosilvestroides : ex. : P. silvestris L. (Europe)

- Parryanoides : ex. : P. Parryana Eng. (Californie)

3. Cembrapinus : feuilles par 5 , haplostélées, euphylles non decurrentes, ombilic terminal.

Ce sons-genre comprend 4 sections :

- Armandioides: ex. : P. Armandi Franchet (Centre W. Chine)

- Parvifloroides : ex. : P. parviflora Sieb. et Zuec. (Japon)

- Stroboides : ex. : P. Strobus L. (Est de l'Amérique du Nord)

- Flexilioides : ex.: P. flexilis James (Mexique, Rocheuses).

La section des Halepensoides, du sous-genre Eupinus, nous intéresse le plus. Le Pinus brutia s'y trouve rattaché.

Dans cette section les trachéides des rayons ont une paroi sinueuse, a dents peu nettes. Les ponctuations des plages sont par 1 ou 2 , chez $P$. longifolia; 1 à 4 chez $P$. halepensis; 2 à 4 chez $P$. leucodermis.

Cette section est divisée en 3 groupes :

1) Un groupe * Heldreichii * des Balkans et du Sud de l'Ttalie, à deux feuilles et cône caduc : $P$. leucodermis Ant. de Calabre, Albanie, Yougoslavie, Grèce et $\boldsymbol{P}$. Heldreichii Christ. d'Albanie et Thessalie.

2) Un groupe halepensis autour de la Méditerranée, passant du cône caduc au cône serotineux ${ }^{(2)}$. C'est un groupe de pins à 2 feuilles qui renforme les $\boldsymbol{P}$. halepensis Mill. et $\boldsymbol{P}$. brutia Ten.

3) Un groupe longifolia à 3 feuilles, à cônes persistants: $P$. longifolia Roxb. et Lamb. des pentes méridionales des Himalayas et $\boldsymbol{P}$. Canariensis $\mathrm{C}$. Smith des Canaries.

(i) Le cône peut être caduc ou persistant plus ou moins longtemps. Dans le dernier cas, il peut s'ouvrir de façon irrégulière dans le temps, on dit qu'il est serotineux, parfois même il reste fermé de nombreuses années. 
En résumé, le Pinus brutia fait partie du groupe "halepensis " de la section des Halepensoides et du sousgenre des Eupinus.

Le Pinus brutia a été décrit pour la première fois par Tenone (botaniste napolitain) en 1811. Après lui, ce pin a été souvent confondu avec le $P$. halepensis Mill. ou considéré comme une simple variété de celui-ci ( $P$. halepensis var. brutia Elwes et Henry 1910).

Certains botanistes l'ont même décrit plusieurs fois sous des noms différents : P. pyrenaica Lap. ; P. Pallasii Parol. ; P. paroliniana Webb; P. parolinii Viv.; P. hispanica Cook.; P. penicillus Lapeyr.; $P$. loiseleuriana Carr., etc.

Après TenoRe, le Pinus brutia n'a jamais plus été retrouvé à l'état spontané dans l'Aspromonte (Calabria), l'ancien "Ager Brutius " des Romains (ALlegri, 1959).

Jusqu'à une date très récente, on confondait le Pin qui habite l'Amanus, le Cassius, le Baer-Bassit et le Liban avec le Pin d'Alep (Gombault, Aperçu sur la Flore de la Syrie et du Liban et de la région d'Antioche, Beyrouth, 1954, p. 132). On peut voir la même confusion dans les Rapports du Département Forestier de Nicosie, Chypre ; ex. : Rapport 949 : "Pinus halepensis variety brutia in Pahos Forest". Dans "Iter Cyprium" de Harald Lindberg, Helsingfors 1946, le Pinus brutia n'est pas mentionné dans la flore de Chypre; on mentionne $P$. halepensis Mill. et $P$. nigra Arnold var. Pallasiana Lamb.

Or, ces deux pins sont bien distincts biologiquement, anatomiquement et morphologiquement et constituent deux espèces bien séparées. Tous les botanistes et dendrologues modernes sont d'accord sur ce point de vue (L. EMBERgER, 1960 ; GaUsSEN, 1960 ; Thiebaut, 1954 ; Mouterde, Bouloumot, Pardé, M. SeLIK, 1959 ; KAYACIK, 1954).

Nos recherches dans le Baer-Bassit, le Cassius et le Djebel Alaouite nous amènent à classer le Pin de ces régions comme Pinus brutia Ten. Nous avons distingué une forme de $P$. brutia très rabougrie sur les serpentines et peridotites pyroxéniques et qui serait proche de la var. Agraphiottii Papaioannou, trouvé à Lesbos et en Anatolia sur serpentine également. Tous les arbres rabougris du Baer-Bassit ne le sont pas tous à cause de la serpentine, car beaucoup d'arbres sont mutilés et déformés par 
l'homme. Ce rabougrissement sur la serpentine serait-il dû à la grande richesse de ces roches en magnesium qui provoque un déséquilibre ionique dans le sol? De plus, ces roches renferment des traces de Nickel $(0,20-0,30 \%$ dans les péridotites à enstatite). Les recherches récentes en Californie (Kruckenberg) ont montré que, sur les serpentines; beaucoup d'espèces ne peuvent y vivre ou s'y développent très mal; elles sont parfois représentées par des écotypes édaphiques. Il n'est pas étonnant que des espèces linnéennes de la flore des serpentines dans le Baer et le Bassit y soient représentées par des écotypes édaphiques.

Il nous est impossible, dans l'état actuel de nos connaissances, de trancher cette question dans notre région; mais la voie est largement ouverte pour des recherches futures.

Dans son aire géographique, le $P$. brutia présente des variétés : GaUssen (1960) distingue les variétés suivantes (leurs caractères seront définis plus loin):

- P. brutia var. eldarica Medw. d'Iran;

- " var. pithyusa Stev. de Thrace et Asie Mineure keviczii $=$ " varitima taurica Stavic) de Crimée.

Enfin, des types climatiques de Svoboda s'ajoutent à ces variétés : dalmatica, graeca, pontica.

\section{Considérations phylétiques}

La famille des Pinacées (Abiétacées) est connue depuis le Jurassique. Le genre Pinus existe depuis le Wealdien (Jurassique supérieur), et les diverses sections ont été reconnues dès le Crétacé inférieur (L. Emberger, 1960). Ce genre est donc très vieux. Il représente le type d'organisation le plus ancien chez les Abiétacées et est considéré comme le type ancestral de ceux-ci. Le pin fut l'ancêtre lointain du Cèdre et du Sapin (GAUSSEN, 1960).

Malgré l'ancienneté du genre et son organisation primitive, certains rameaux ont eu une longue destinée les amenant à des types d'évolution poussée dans certains de leurs organes (ex.: $P$. halepensis, $P$. brutia, $P$. canariensis) ; le $P$. aristata Engel. des Montagnes Rocheuses, paraît le plus évolué des pins actuels (surévolution?) peut être à cause du milieu hostile dans lequel il vit 
qui a accéléré son évolution. Le sous-genre Eupinus parất le plus primitif par son appareil végétatif.

GaUSSEN considère les sections des Merkusioides, Halepensoides et Khasyosilvestroides comme ayant une lointaine origine commune eurasiatique, mais ce qui est plus net ce sont les relations des sections Halepensoides et Khasyosilvestroides par un ancêtre ressemblant plus ou moins au type Laricio.

On attribue una grande importance à l'étude chromosomique pour établir les relations phylogéniques. Les travaux de Mme VABRE-DuRRIEU sur les noyaux quiescents ont déjà donné quelques résultats (1958). Elle propose de considérer comme primitifs : Pinus densiflora, $P$. nigra, $P$. silvestris, $P$. uncinata ; plus évolués : P. Banksiana, P. radiata ; évolnés: P. Pinaster, P. Sabineana ; très évolués: $P$. halepensis, $P$. brutia, $P$. canariensis, $P$. pinea. Cette considération coincide avec les résultats obtenus par Gaussen où les Pinus halepensis et brutia sont classés comme des pins évolués.

Gaussen prend en considération la position des canaux résinifères dans les aiguilles et l'aile des graines car il y a corrélation entre ces deux caractères : canaux marginaux et disparition de l'aile sont des caractères évolués. La dimension des grains de pollen est également considérée. L'évolution des pollens est vers la croissance de taille. Chez le $\boldsymbol{P}$. brutia les canaux résinifères sont marginaux ou submarginaux et la dimension des grains de pollen est $54 \mu$.

Les $P$. halepensis et brutia sont voisins dans la série phylétique. Ils s'hybrident entre eux (Papaioannov, 1954). On a trouvé 3 pins fossiles an Miocène ressemblant au Pinus brutia: $P$. saturni Unger (Croatie); P. sarmatica Palib. (Péninsule de Kertsch); P. Wassoewiczi M.V. (sud de la Russie: Tehiatouri).

H. Czeczot (1954) considère le $P$. saturni comme "indubitable predecessor " du $P$. brutia actuel. Enfin, d'après Gaussen, le Pinus brutia aurait existé au Tertiaire jusqu'en Provence, en France et le Pinus halepensis jusqu'en Bohême.

\section{Caractères botaniques et dendrologiques}

Le Pinus brutia est un grand arbre pouvant atteindre 30 à 35 mètres de haut. Son fût est droit et élancé, sa cime est régu- 
lière, dense, d'un vert foncé, conique mais devenant tabulaire quand l'arbre avance dans l'âge. L'arrêt de la croissance de la cime en hauteur est fonction de la profondeur et de la fertilité des sols. Sur les sols profonds, sur gabbros et dolérites, la cime continue à se développer beaucoup plus longtemps que sur les serpentines très superficielles. L'écorce est très épaisse dans la partie inférieure du fût et possède de longs et profonds sillons.

Les rameaux sont plus ou moins fins de 0,4 à $0,5 \mathrm{~cm}$, jaune rougeâtre ou vert. Les bourgeons sont allongés, non résineux et à écailles libres. Les feuilles sont par 2 , de couleur vert foncé, assez rigides, non groupées en pinceaux, serrulées et ayant 12 ̀े $18(23) \mathrm{cm}$ de long et plus de $1 \mathrm{~mm}$ de large. Les fleurs sont unisexuées, monoïques. Les mâles en touffes, dressées, oblongues, $1,2 \mathrm{~cm}$ de long, de couleur orangé; pollen $54 \mu$. Les cônes sont de forme ovale, de 5 à $11 \mathrm{~cm}$, sans pédoncule ou à pédoncule court, mais non recourbé ; ils sont dirigés vers le haut ou perpendiculaires au rameau. Les écailles sont à rayures étoilées, jusqu'à $2 \mathrm{~cm}$ de large, avec apophyse plus ou moins saillante; l'ombilic est gros et presque plat. Les graines sont ailées. Le kilogramme de graines renferme 20.000 graines environ (contre 50.000 env. chez le Pinus halepensis).

L'arbre commence à fructifier assez tôt, vers l'âge de 10 à 12 ans, mais les graines ne sont bien formées qu'à partir de l'âge de 18 à 20 ans.

Les différentes variétés peuvent être distinguées de la façon suivante :

- fenilles épaisees, serrées contre le rameau, de 8 d $10 \mathrm{~cm}$ de long; cônes drassés sur un pédoncule court, les jeunes plus ou moins sphériques, les mûrs ovales allongés de 5 a $6 \mathrm{~cm}$ de long, brun clair, apophyse convexe : $P$. brutia var. eldarica Medw.

- feuilles ayant 10 a $15 \mathrm{~cm}$ de long sur $0,1 \mathrm{~cm}$ de large, robustes. Côue courtement pédonculé, ovale, 6 à $10 \times 4$ à $5 \mathrm{~cm}$; plus court que les feuilles, brun rougestre ; ́cailles presque plates ; ombilic large, deprimé, gris : $P$. brutia var. pithyusa Stev.

- feuilles de 13 a $20 \mathrm{~cm}$; cône ovale, pointu ; jaune ; écailles carénées ; orabilic elliptique, profondément enfoncé : $\mathcal{P}$. brutia var. Stankeviezii Sukac.

Le bois de $P$. brutia est assez lourd, dur et de qualité moyenne. Il est plus recherché que celui du pin d'Alep à cause de la rectitude de son fût qui donne plus de bois d'oeuvre. Le bois trouve des débouchés assez variés : poteaux télégraphiques, caisserie, pâte à 
papier, etc. Son écorce renferme une matière tannante, ntilisée autrefois par les anciens pour le tannage.

Le bois renferme une résine de bonne qualité ; l'essence est un mélange de $\alpha$ pinène, $\beta$ pinène et $d-\Delta^{3}$ carène (uniquement pinène chez $P$. halepensis); elle est lévogyre. Les expériences sur le gemmage des pins faites dans le Baer-Bassit à $450 \mathrm{~m}$ d'altitude ont montré que l'arbre donnait en moyenne $1,5 \mathrm{~kg}$ par campagne, donc moins que le $P$. halepensis qui donne en Grèce 3,200 à $4,800 \mathrm{~kg}$ suivant les stations (J. C. Papaioannou, 1954).

\section{Les peuplements élites de Pinus brutia dans le Baer-Bassit}

Les peuplements de $P$. brutia ne sont pas homogènes dans le Baer et le Bassit. Certains arbres sont constitués d'arbres très élancés, peu branchus et à cime conique et en flèche ; d'autres, au contraire, ayant le même âge, ayant subi le même traitement et poussant sur les mêmes sols, sont constitués d'arbres trapus, à cime étalée et sont très branchus. En outre, ces peuplements couvrent une superficie assez importante, ce qui nous permet de penser que ces caractères sont les caractères moyens de tous les arbres du peuplement et sont aussi héréditaires, c'est-à-dire qu'on peut considérer comme fortement probable ou même comme certaine la transmission de ces qualités des pieds mères à leurs descendants.

A Froulok, dans le Baer, existe un peuplement d'une vingtaine d'hectares, situé sur roches vertes, entre 400 et $450 \mathrm{~m}$ d'altitude; la pluviométrie moyenne annuelle est de l'ordre de 900 à $1000 \mathrm{~mm}$. Le sous-étage est bien développé et constitué de : Quercus pseudocerris, Pistacia palaestina, Ostrya carpinifolia, Carpinus orientalis, Phillyrea media, etc. Sans parler de race élite au sens strict, nous appelons ce peuplement "peuplement élite"; les caractères des arbres qui le constituent sont les suivants :

\footnotetext{
Port : élancé, fût droit, cime conique en flèche

Branches : peu nombreuses, fines, courtes, régulières et dirigées légèrement vers le haut

Trone : rectiligne, cylindrique, sans noeud, se continuant distinctement jusqu'à la flèche. La hauteur du fût élagué atteint 10-15 m.

Aiguille : de couleur vert foncé

Groissance : assez rapide.
} 
I. NAHAL, Contribution à l'étude de la végétation du Baer-Bassit et du Dj. Alaouite (Şyrie).

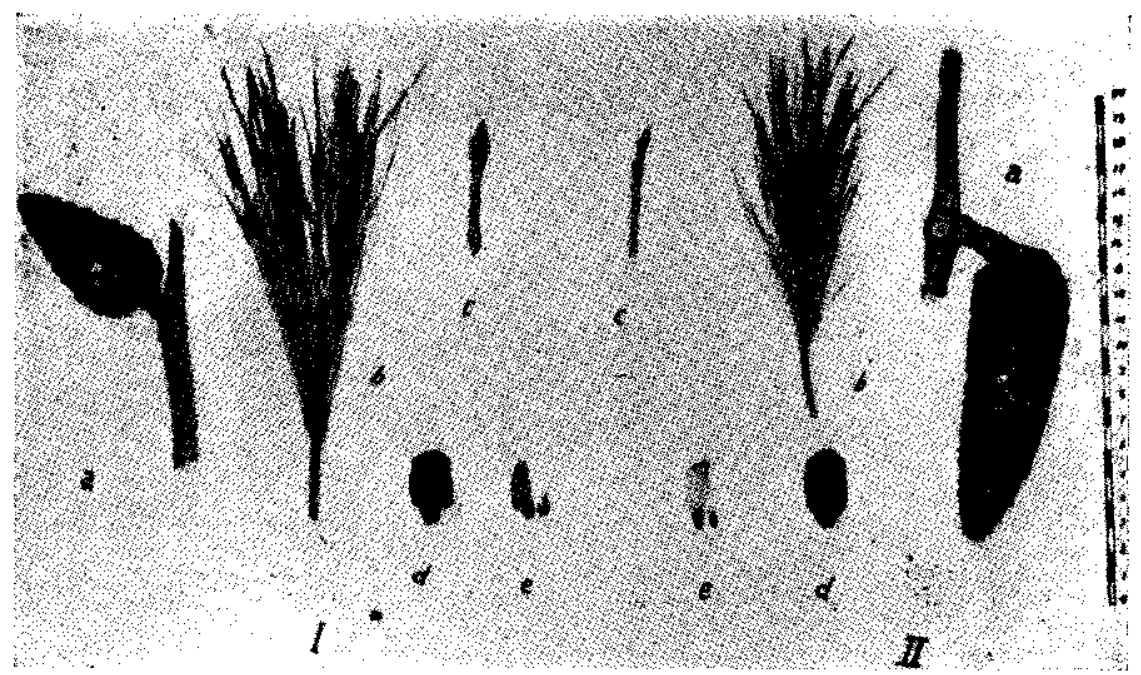

Fig. 6 - I : Pinus brutia Ten. II : Pinus halepensis Mill. - $a$ : cône. $b$ : ra mean feuillé. $c$ : bourgeon. $d$ : écaille. e: graine avec l'aile. (Photo Kayacik)

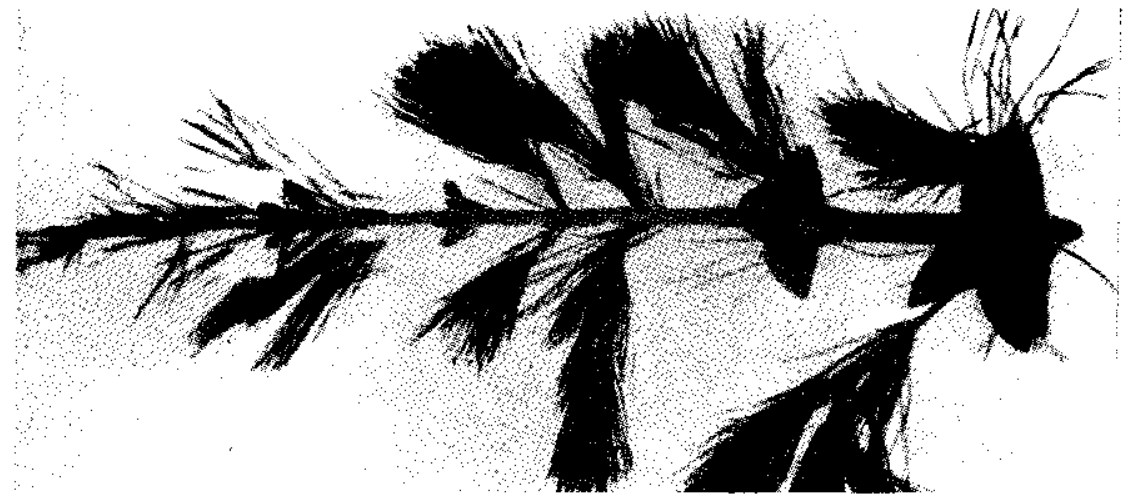

Fig. 7 - Rameau de Pinus brutia Ten. 
Il est bien entendu que ces caractéristique sont celles de l'arbre moyen et qu'on peut trouver dans des peuplements non classés "élites" des beaux arbres, mais dans ce cas on est beaucoup moins sûr de la transmission héréditaire de ces caractères.

On a identifié un autre "peuplement élite "à Quarankoul dans le Bassit, à une altitude plus basse et sur roches vertes. Le sousétage est bien développé et constitué de : Quercus calliprinos, $P \boldsymbol{i}$ stacia palaestina, Fontanesia phillyreoides, Phillyrea media, etc.

Ces deux peuplements élites de Froulok et de Qarankoul doivent être traités en peuplements semenciers afin de récolter les graines nécessaires aux reboisements. Le peuplement élite de Froulok qui est relativement avancé dans l'âge, doit être éclairci afin de permettre un large développement des cimes des arbres et par suite une abondante fructification. Le peuplement élite de Qarankoul, beaucoup plus jeune et qui était à l'état très serré, sera éclairci graduellement; une éclaircie trop forte pourrait y provoquer des chablis, mais ce peuplement a beaucoup d'avenir à cause de son jeune âge.

A l'intérieur de ces deux peuplements élites on peut choisir des arbres qui présentent l'optimum des qualités de formes, de santé et de vigueur et auquel on donnera le nom "d'arbres plus"; ceux-ci seront numérotés et suivis de prés et serviront comme porte-graines pour la récolte de petites quantités de graines destinées à la création future de vergers à graines ou à l'expérimentation.

Il est à conseiller d'éviter les arbres de bordures (qui sont en général les plus productifs) afin d'éviter les hybridations possibles qui auraient pu se produire au contact de ces peuplements elites avec les peuplements voisins.

Les graines nécessaires aux reboisements sont, jusqu'à maintenant, récoltées en Syrie, sur des arbres de mauvaises formes, isolés, mais où les cônes sont très nombreux et faciles à atteindre et sans soucis de leur patrimoine héréditaire.

Cette pratique est à déconseiller énergiquement et même à interdire car les peuplements artificiels issus de ces graines seront de mauvaise qualité, done de peu de valeur économique, le marché de bois actuellement étant en faveur des bois de qualité, de forme régulière et plus ou moins cylindrique. 


\section{Autobeologie du Pinus brutia dans le Nord-Onest de la Syrie}

\subsection{Altitude ET EXPosition}

On trouve le Pinus brutia dans le Baer-Bassit, le Cassius et le Djebel Alaouite, mais e'est dans le Baer--Bassit qu'il constitue de belles forêts, denses, et économiquement exploitables. Dans toutes ces régions, on le trouve dès le niveau de la mer et il monte jusqu'à $900 \mathrm{~m}$ d'altitude. Dans le Bassit, les forêts de $P$. brutia côtoient la mer.

D'après Chapman, le $P$. brutia se rencontre à Chypre jusqu'à $1600 \mathrm{~m}$ et d'après P. MouTERDE, jusqu'à $1800 \mathrm{~m}$ au Liban.

On le rencontre sur toutes les expositions. Il est vrai que les forêts sur les expositions Ouest sont les meilleures mais ceci est dû aux vents chargés d'humidité de direction Ouest et Sud-Ouest et à l'insolation plus faible de ces expositions qui a pour effet de diminuer la perte en eau du sol par évaporation.

\subsection{Conditions cumatiques}

Le $P$. brutia résiste bien aux basses températures. Il peut supporter des températures de -15 à $-18^{\circ} \mathrm{C}$. Des plantations artificielles de $\boldsymbol{P}$. brutia ayant 30 à $\mathbf{5 0}$ ans d'âge existent à Slenfé, à $1000 \mathrm{~m}$ d'altitude et où la moyenne des minima du mois le plus froid $(\mathrm{m})$ varie entre 1 et 2 . Certaines années $(\mathrm{m})$ descend audessous de zéro; en $1959(\mathrm{~m})$ est descendu à $-3,3^{\circ} \mathrm{C}$. Les forestiers grees ont également remarqué la résistance au froid de $P$. brutia qui dépasse celle de $P$. halepensis.

Dans le Baer-Bassit on trouve le $P$. brutia dans l'étage méditerranéen bioclimatique subhumide et humide. Dans le Djebel Akraad, à l'intérieur, on le rencontre dans l'étage semi aride.

\subsection{CONDITIONS EDAPHIQUES}

On trouve le $P$. brutia sur des roches très variées aussi bien siliceuses que calcaires. Dans le Baer-Bassit et le Djebel Alaouite, on le trouve sur : péridotites pyroxéniques, gabbros, diorites, dolérites, marnes crayeuses, argile rouge et radiolarites; mais beau- 
coup plus rarement sur calcaire compact. C'est une espèce indifférente à la composition chimique des sols.

Par contre, la composition physique des sols et leur structure sont très importantes pour la régénération naturelle du Pinus brutia. Nous avons remarqué que ce dernier se régénérait très facilement dans le Baer et le Bassit sur les sols issus des roches vertes et sur les marnes crayenses. Par contre, sa régénération est plus difficile sur la Terra rossa, sauf si certaines années, exceptionnellement humides, se succèdent; la Terra rossa devient trop sèche en surface pendant la période estivale et constitue ainsi une croûte superficielle défavorable à l'alimentation en eau des semis. Le $P$. brutia s'accomode de sols très superficiels et, grâce à son système radiculaire puissant, il arrive à s'insinuer même dans les petites fissures des serpentines pour chercher l'eau et les sels minéraux.

\section{Tempérament du Pinus brutia}

Le $P$. brutia est une essence de lumière, ses semis ne se développent bien qu'en plein découvert. Toutefois, un léger abri est très utile aux jeunes semis en les protégeant contre la dessication, surtout dans notre région où l'été est très sec et très chaud. Grâce à ce tempérament, le $P$. brutia peut coloniser les places vides dans les associations voisines. C'est ainsi qu'il a conquis l'étage du Ceratonieto-Lentiscetum et arrive ainsi jusqu'à la mer. Il a également conquis, dans certaines régions, le PistacietoQuercetum calliprini (garrique à Pistacia palaestina et Quereus calliprinos) et même certaines zones du Quercetum pseudocerridis dans le Baer, dont nous parlerons plus loin.

\section{Types de peuplements de Pinus brutia dans le Nord-Ouest de la Syrie}

On peut, en gros, répartir les types de peuplements de Pinus brutia dans le Nord-Ouest de la Syrie en 3 catégories :

8.1. Futaie pure régulière

8.2. Futaie pure jardinée

8.3. Peuplements mélangés. 


\subsection{LA FUTAIE PURE REGULI'̀re}

C'est le type de peuplement le moins répandu dans notre région ; mais il n'est pas non plus très rare. On en tronve de très beaux dans les régions de Froulok, Qarankoul, Qastal Moaf, Chaukarane. Un sous-étage de Quercus pseudocerris, Quercus infectoria, Quercus calliprinos, Pistacia palaestina, etc. est assez fréquent sous ces peuplements.

Bien que peu répandu, à l'état actuel, ce type de peuplement doit être adopté partout où la croissance est rapide, c'est-à dire sur les bonnes stations, et où la régénération naturelle est facile et les incendies relativement peu fréquents. Son aménagement est simple et facile à appliquer et il est le seul à parmettre d'obtenir des arbres à fûts droits et réguliers et de favoriser le développement d'un léger sous-étage. La durée de la révolution sera fixée d'après le nombre d'années nécessaires pour arriver à la dimension d'exploitabilité de bois d'oeuvre, soit $40 \mathrm{~cm}$ de diamètre $(1,20 \mathrm{~m}$ de circonférence), et variera entre 80 et 100 ans suivant la fertilité des stations (en moyenne 75 ans).

\subsection{La FUTAIE PURE JARdinÉE}

Ce type de peuplement est de loin le plus répandu dans le Baer-Bassit et le Djebel Alaouite. Il représente, en effet, l'état de nos forêts tel qu'il résulte des incendies, des dégâts dus à l'homme, de l'absence d'une sylviculture appropriée et d'aménagement convenable.

Ces peuplements présentent le plus souvent un jardinage par trouées résultant du fait de l'action de l'homme et du feu. Ils sont irréguliers, hétérogènes comme répartition des âges et des diamètres, étant donné qu'ils n'ont pas été aménagés et qu'ils n'ont jamais été soumis à un traitement rationnel avant une époque très récente. On y trouve des arbres de tous âges de 1 ̀े 150 ans et plus. De même, le nombre de sujets à l'hectare est très variable. Le mélange des arbres des diverses classes n'obéit à aucune règle et présente un grand nombre d'aspects; les sujets 
âgés ou d'âge moyen sont parfois très isolés les uns des autres, d'autres fois groupés sur plusieurs ares.

Le traitement de ces peuplements est plus difficile et nécessite le recours $d u$ forestièr à un aménagement provisoire ou de transition de durée courte ( 30 à 40 ans), permettant d'introduire de l'ordre dans la composition et la régénération des peuplements, avant l'application d'un aménagement normal ou définitif.

Dans ces peuplements irréguliers, le sous-bois inflammable est très fréquent et le bois est de qualité médiocre. Il est à abandonner là où on peut faire de la futaie régulière.

\subsection{Les PEUPLEMENTS Mállangés}

Nous avons vu que, grâce à son pouvoir de colonisation, le pin brutia envahissait d'autres associations. On le trouve dans le Baer-Bassit et le Djebel Alaouite installé dans l'association du Quercus calliprinos et Pistacia palaestina et dans le Quercetum pseudo-cerridis et constitue ainsi, avec ces espèces, des peuplements mélangés.

\subsubsection{Peuplements de Pinus brutia et Quercus pseudocerris}

Les peuplements mélangés de Pinus brutia et Quercus pseudocerris sont assez fréquents dans le Baer et le Bassit à partir de $400 \mathrm{~m}$ d'altitude et spécialement sur les expositions fraiches et humides, car ce sont les expositions préférées pour le Quercus pseudocerris. Dans ces peuplements, le Pinus brutia est très beau ; sont fût est droit, élancé et bien élagué. C'est le chêne qui le moule et permet l'élagage de ses branches. Ces peuplements se trouvent aussi bien sur péridotites pyroxéniques et serpentines que sur gabbros, diorites et dolérites. Mais, sur ces derniers, où les sols sont plus profonds, on doit chercher un équilibre entre les deux essences. Le traitement sera un peu plus difficile que dans les peuplements purs, mais ce mélange de résineux et feuillus sera très favorable à la vie biologique des sols en améliorant la nature de l'humus et les rendra moins vulnérables aux incendies 
et aux prédateurs. Par contre, sur les sols superficiels dérivant des serpentines et des péridotites, il faut donner la préférence au Pinus brutia qui s'adapte mieux à ces sols et y pousse même très bien. Sur ces sols superficiels, le Quercus pseudocerris pousse très mal. Mais, favoriser le Pinus brutia, ne veut pas dire, chasser totalement le Quercus pseudocerris de ces stations. On a même intérêt à le laisser, mais en sous-étage comme on le verra plus loin.

Sur les sols profonds, le peuplement sera traité en futaie régulière (pour les deux essences). Dans le deuxième cas, on aura une sorte de futaie sur taillis, le pin constituant la futaie et le chêne pseudocerris le taillis.

\subsubsection{Peuplements de Pinus brutia et Querous calliprinos}

Dans ces peuplements, le Pinus brutia constitue l'étage dominant et le Quercus calliprinos l'étage dominé. C'est une futaie. de pins sur taillis de chêne. Le traitement de ces peuplements doit être orienté dans le sens de maintenir le taillis sous forme de sous-étage. Ce dernier sera recépé au moment de la coupe d'ensemencement.

L'enrésinement des taillis de chêne Kermès doit être favorisé par le forestier, étant donné la supériorité du pin brutia sur le chêne Kermès comme bois d'oeuvre.

La méthode à suivre, dans le traitement de ces peuplements est la suivante :

Par recépage du taillis on dégagera les semis de pins étouffés dans la souille et qui prendront vite le dessus sur les rejets de souches du taillis, étant donné la croissance plus rapide des pins. Par la suite, on continuera, lors des éclaircies et dépressages de taillis, à dégager les jeunes sujets de pin brutia, dont le nombre ne fera que crôtre. Lorsque l'enrésinement sera suffisant et que les pins auront l'âge de donner des graines fertiles on abattra graduellement les semenciers. On peut escompter qu'après 40 à 50 ans, on pourra obtenir un peuplement de futaie sur taillis où. l'étage dominant sera constitué par des réserves de pins généralement de même âge que le chêne, mais ayant poussé plus vite que lui. 


\section{Importance du sous-étage de feuillus dans les forêts de Pinus brutia}

Nous avons insisté plus haut sur la nécessité du maintien d'un sous-étage de feuillus sous les peuplements de pin brutia et sur l'avantage d'un mélange des essences résineuses et feuillues. En effet, le sous-étage a des avantages multiples dans notre région que nous allons essayer d'analyser dans ce qui suit :

1) Sous un climat méditerranéen comme le nôtre où l'évaporation est très intense en été à cause de la sécheresse et de la chaleur excessives, le sous-étage joue le rôle d'un écran qui protège le sol contre les irradiations solaires directes, diminuant ainsi les pertes d'eau du sol par évaporation. Ce sous-étage sert donc ì l'économie de l'eau dans le sol; il empêche son gaspillage, e'est un avantage très important dans une région où l'eau est très précieuse et de laquelle dépend la vie végétale, animale et humaine.

2) Le sons-étage protège le sol, dans les futaies claires, contre l'érosion pluviale et contre la destruction de la structure, en particulier dans notre région où les pluies sont très brutales et tombent en averses.

3) Le sous-étage qui est constitué, le plus souvent, par un mélange d'espèces, est particulièrement favorable à la vie biologique des sols : un mélange d'essences donnera un humus plus actif qu'un peuplement pur de pins. En outre, ce mélange d'essences a une influence favorable sur la remontée biologique des cations puisés en profondeur, dans la roche-mère en voie de décomposition et ramenés en surface par la chute des feuilles. Dans le cas du mélange Pinus brutia-Quercus psendocerris sur les roches vertes, cette remontée est appréciable pour deux raisons : 1) la rochemère est riche en cations; 2) le Quercus pseudocerris, étant un arbre à feuilles très larges et caduques, la quantité des cations puissées dans la roche mère en décomposition est assez appréciable.

4) Les forêts de Pinus brutia sont particulièrement sensibles à l'incendie qui y causent des dommages considérables. C'est la nature du bois de pin brutia, plus ou moins imprégné de résine et la propagation du feu par les cônes projetés au loin qui favorisent la marche de l'incendie. Les peuplements mélangés ping- 
feuillus, ou la présence d'un sous-étage de feuillus, rendront les peuplements bien moins vulnérables. Le Pinus brutia est l'essence la plus importante de la Syrie; c'est pourquoi toutes les dispositions doivent être prises pour protéger ses peuplements contre le feu, dans toute la mesure du possible, tant en favorisant le mélange des essences, la création d'un sous-étage, l'établissement de pare-feux, qu'en assurant la rapidité des secours par l'ouverture de routes et de chemins à l'intérieur des massifs de pins et l'organisation du service de lutte contre l'incendie.

\section{II - CEDRUS LIBANI A. Rieh. (1823) \\ Nom arabe: Al Arz}

\section{Aire geographique du genre Cedrus (Trew.) Link 1841}

Le genre Cedrus est très ancien, d'après GaUsSEN (1950-52) les genres Abies et Cedrus ont un lointain ancêtre commun. A partir de cet ancêtre qu'on peut appeler $\mathrm{A}$, le phyllum $\mathrm{A}$ Cedrus s'est moins éloigné des caractères de l'ancêtre que le phyllum $\mathbf{A}$ Abies. Autrement dit, les Cedrus actuels ressemblent plus à l'ancêtre commun que les Abies actuels.

Pour l'origine de Cedrus, il semble d'après VaN CaMPo et DE FerRe qu'on puisse la rechercher dans les ancêtres du Pinus Gerardiana du groupe des Paracembra.

L'existence du Cedrus au tertiaire est certaine. Il occupait alors des régions plus vastes que celles qu'il occupe à l'heure actuelle. On a retronvé des fossiles dans le Miocène du Vivarais ( $C$. vivarensis) et dans le Pliocène d'Auvergne; on a trouvé des restes de Cedrus dans la province d'Tenisseisk en Sibérie, à $56^{\circ}$ de latitude; le genre Cedrus a été indentifié par le pollen dans le Villafranchien (limite entre le Tertiaire et le Quaternaire) des Alpes bergamasques et dans le Quaternaire du Sahara (A. Pons et P. QuEZEL).

Actuellement, le genre Cedrus a une aire géographique localisée et très morcelée. Il est complètement absent en Europe, au Nord de la Méditerranée, à l'état spontané du moins. Ce sont 
probablement les glaciers qui ont refoulé le Cedre de l'Europe au sud de la Méditerranée. Il n'a sans doute pas pu, étant avant tout montagnard, repasser en Europe comme l'a fait son associé le chêne vert, plus plastique, sa place ayant été prise dans sa station par une essence plus rustique. Néanmoins, réintroduit artificiellement en Europe par les forestiers, il s'y comporte d'une façon admirable et se régénère naturellement avec facilité comme le montrent les reboisements du Mont Ventoux dans le sud de la France.

Son aire géographique actuelle s'étend de l'Himalaya jusqu'à l'Atlas marocain en passant par le Taurus et l'Amanus (Turquie), le Djebel Alaouite (Syrie), l'nle de Chypre et le Liban. Il manque en Palestine, Egypte, Lybie, et Tunisie.

Au milieu de cette aire, le Cedrus forme trois blocs d'inégale importance et à l'intérieur de ces blocs il se présente en massifs le plus souvent éloignés les uns des autres, notamment en Asie Mineure et en Algérie :

1) Le bloc le plus asiatique habite le Nord-Onest des montagnes de l'Himalaya (Indes, Pakistan et Afghanistan) entre 1300 et $3500 \mathrm{~m}$ d'altitude et où il constitue de très vastes forêts. C'est le groupe des Cèdres Déodars, qu'on trouve à l'état pur ou mélangé à d'autres espèces, principalement: Pinus excelsa Wall., Pinus longifolia Roxb., Picea morinda Link., Abies Pindrow. C'est le groupe le plus important de tous.

2) Le deuxième bloc, que nous appelons "groupe des Cèdres du Liban ", habite les montagnes de la Méditerranée orientale (Taurus, Amanus, Djebel Alaouite, Liban). A ce groupe nous rattachons les boisements de Cèdres de l'Ile de Chypre qui couvraient autrefois les hautes altitudes des montagnes, mais très réduits actuellement et ne compte plus que quelques milliers d'individus occupant certaines montagnes dans la partie sud-onest de l'Ile, où ils s'étagent entre 1300 et $1700 \mathrm{~m}$. Le Cedre de Chypre croit par petits groupes au milieu d'un chêne à feuilles persistantes endémiques, le Quercus alnifolia. Il est également accompagné des espèces suivantes: Arbutus Andrachne, Acer orientale var. obtusifolium, Laurus nobilis, Clematis cirrhosa, Lonicera etrusca, Crataegus Azarolus, etc.

3) Le troisième bloc est africain et constitue le groupe des 
Cèdres de l'Atlas. Il habite les montagnes de l'Algérie et du Maroc. Il est absent en Tunisie.

En Algérie, l'aire du Cèdre est très disjointe et présente, depuis le Sud-Est du Département de Constantine jusqu'à la limite occidentale de celui d'Alger, huit à neuf stations aux conditions écologiques très différentes (P. Boudy). Sur le versant Nord de l'Atlas tellien (Blida) le Cèdre commence à apparaître vers 1300 à $1400 \mathrm{~m}$ (au versant Sud, il débute à 1400). Dans l'Aurès, il part de 1400 à $1500 \mathrm{~m}$ pour monter à $2200 \mathrm{~m}$.

L'aire $d u$ Cèdre présente ensuite un hiatus de plus de $600 \mathrm{~km}$, jusqu'au groupe marocain qui comprend deux masses distinctes séparées par le détroit sud Rifain. Sa limite altitudinale inférieure au Maroc est capricieuse ; elle est de $1350 \mathrm{~m}$ dans le Rif, 1500 dans le Ktama et 1800 dans les Djebala.

De l'avis de P. Boudy l'optimum écologique du Cèdre en Afrique du Nord est le climat méditerranéen humide et froid, entre 1800 et $2000 \mathrm{~m}$.

En Algérie, le Cèdre apparaît comme étant plus xérophile qu'au Maroc.

\section{Systématique du genre Cedrus (Trew.) Link}

Nous adoptons, pour notre étude, la classification utilisée par PaRdé pour les conifères (Les Conifères, 19555).

Le genre Cedrus appartient à la famille des Pinacées ou des Abiétacées, à la sous-famille des Abiétoidées, à la Tribu des Abiétées, à la sous-tribu des Laricinées.

Certains auteurs (JAHANDIEZ, MarRe, 1931) n'attribuaient au genre Cedrus qu'une seule espèce, le Cedrus libanotica Link, avec, comme sous-espèces ou races, les Cèdres qui habitent l'Himalaya, l'Ile de Chypre et l'Afrique du Nord.

Actuellement, les botaniques et les dendrologues ont tendance à distinguer dans le genre Cedrus quatre espèces distinctes. Pardte (Les Conifères, 1955) distingue dans le genre Cedrus Link les espèces suivantes :

- Cedrus Libani Barr. (1838) qui habite le Taurus, l'Amanus, le Djebel Alaouite et le Liban 
- Oedrus atlantica Manetti (1844) (C. argentea Land. [1855]) qui habite les montagnes de l'Algérie et du Maroc

- Cedrus Deodara Loud. (1838) qui habite le Nord-Ouest de l'Himalaya

- Cedrus brevifolia Henry (1908) qui habite les montagnes de l'lle de Chypre.

Il nous semble que la première appellation valable pour le Cèdre du Liban soit celle qui a été donnée par A. RICHARD en 1823 (Cedrus libani A. Rich. in Dict. class. Hist. Nat. iii, 299, 1823).

En 1825 il fut décrit par Jussieu sous le nom de Cedrus libanensis (in Mem. Mus. Hist. Nat. Paris, xiii, 71 [1825]). Plus tard il a été connu sous des noms divers : Cedrus africana Gord. ex Knight, C. elegans Knight, C. argentea Hort. ex Carr., C. libani Barr. (1838), C. patula C. Koch, C. indica Chambr., ete.

L. EMberger (Arbres du Maroc, 1938), pense que ces divers Cèdres sont des descendants d'un ancêtre unique tertiaire. Les liens génétiques se sont relachés au cours des temps mais n'ont pas disparu et tous les Cèdres sont encore cousins. En effet, les faits géographiques et les caractères morphologiques remarquablement constants qui font de chacun de ces Cèdres des formes bien individualisées sont en faveur de cette manière de voir.

On peut penser que c'est l'isolement géographique qui a favorisé l'évolution du Cèdre ancestral pour donner les Cèdres actuels. C'est bien la séparation de l'Ile de Chypre du continent au Pliocène et son isolement géographique qui a favorisé l'apparition du Cedrus brevifolia, espèce distincte du Cedrus libani qui vient dans les mêmes conditions dans les montagnes voisines (Liban, Alaouite, etc.).

Ces quatre espèces de Cedrus sont des "endémiques" dans les régions où ils vivent.

\section{Caractères botaniques et dendrologiques}

Le Cedrus libani est un grand arbre pouvant atteindre $40 \mathrm{~m}$ de haut, parfois plus. C'est par excellence l'essence noble du Moyen-Orient qui, durant des millénaires, a rendu de grands services aux premières civilisations de la vallée du Nil et du Proche Orient.

Son fût est trapu ; son écorce est épaisse, très rugueuse et 
d'un gris noirâtre ; de profonds sillons la parcourent. Ses branches sont longues et ne sont pas étagées en verticilles comme chez l'Abies eilicica; elles naissent isolément sur le trone et portent une multitude de rameaux courts, glabres ou pubescents, et non pendants mais tous sur le même plan.

Le fût est terminé par une flèche grêle mais robuste. Cette flèche disparaît quand l'arbre avance dans l'âge et la cime devient tabulaire. L'arrêt de cette flèche est plus ou moins tardif suivant la profondeur et la richesse du sol. Les vieux arbres qu'on trouve dans le Nabi Matta sur les sols superficiels, rocailleux, ont une cime très tabulaire, contrastant avec la cime en flèche des jeunes arbres de 30-50 ans.

Le système radiculaire du Cedrus libani est rarement pivotant. Il est plus souvent traçant et s'insinue dans les fissures des roches comme c'est le cas dans le Nabi Matta, mais l'enracinement est robuste lui permettant de se fixer partout.

Les feuilles sont longues de 15 à 35 millimètres, large de $1 \mathrm{~mm}$ et ordinairement d'un vert foncé (la variété "glauca " est très fréquente dans la Cédraie de Nabi Matta). Ses feuilles sont groupées au sommet de très courts rameaux en rosette et vivent généralement trois ans.

Les fleurs sont unisexuées, monoïques et raissent en automne ; les mâles sont d'un vert teinté de jaune, les femelles sont pourpres.

La fécondation des fleurs a lieu à l'automne; durant l'année suivante le cône se forme et à l'automne de la deuxième année reste vert sur l'arbre. Durant la troisième année, il achève de mûrir deux ans après la fécondation.

Le fruit est un cône dressé à sommet tronqué, de 7 à $10 \mathrm{~cm}$ de long, large de 4 à $7 \mathrm{~cm}$, d'abord d'un pourpre violacé, puis d'un gris verdâtre avant maturité. Il est composé d'écailles étroitement imbriquées.

Chaque écaille fertile abrite deux graines ailées. Le cône s'ouvre au début de l'hiver en se désarticulant; les graines sont libérées et seul l'axe du fruit persiste assez longtemps sur l'arbre. La déhiscence des cônes se fait, le plus souvent, par l'humidité et au besoin par l'immersion dans l'eau. Cependant, le cône mûr s'ouvre fréquemment au soleil. 
I. NaHAL. Contribution à l'étude de la végétation du Baer-bassit et du 1)j. Alaouite (Syrie).

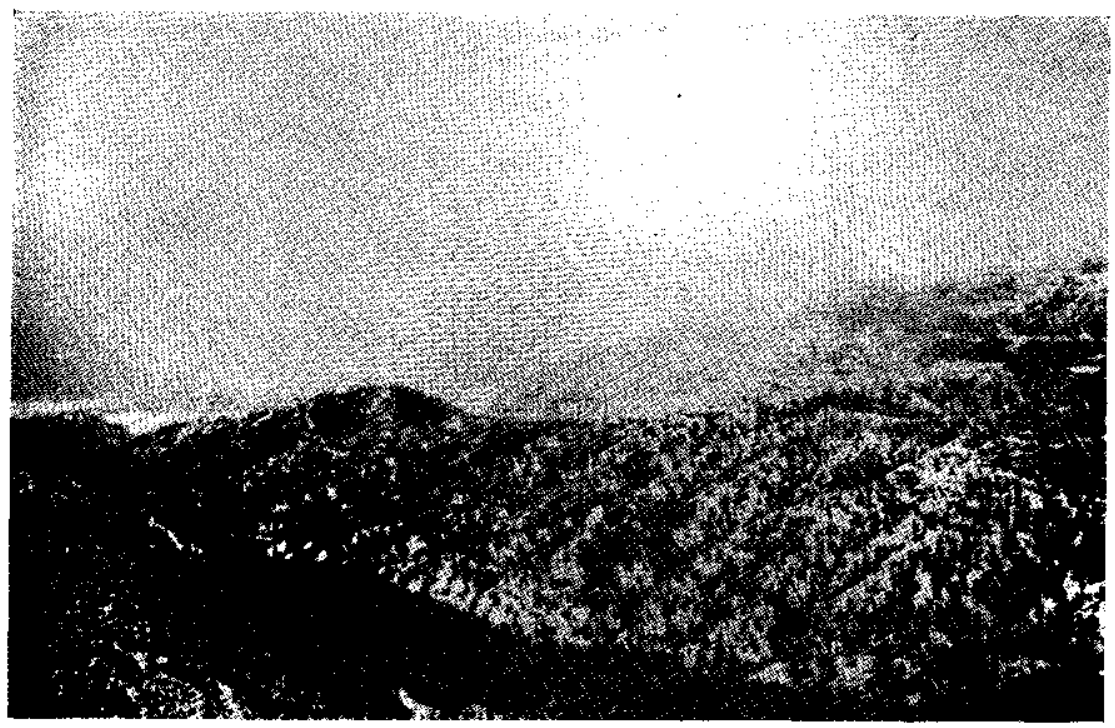

Fig. 8 - Forêts de Pinus brutia Ten. sur péridotites pyroxéniques et serpentine dans le Bassit. Contraste de végétation entre les expositions $\mathrm{N}$ et $\mathrm{S}$.

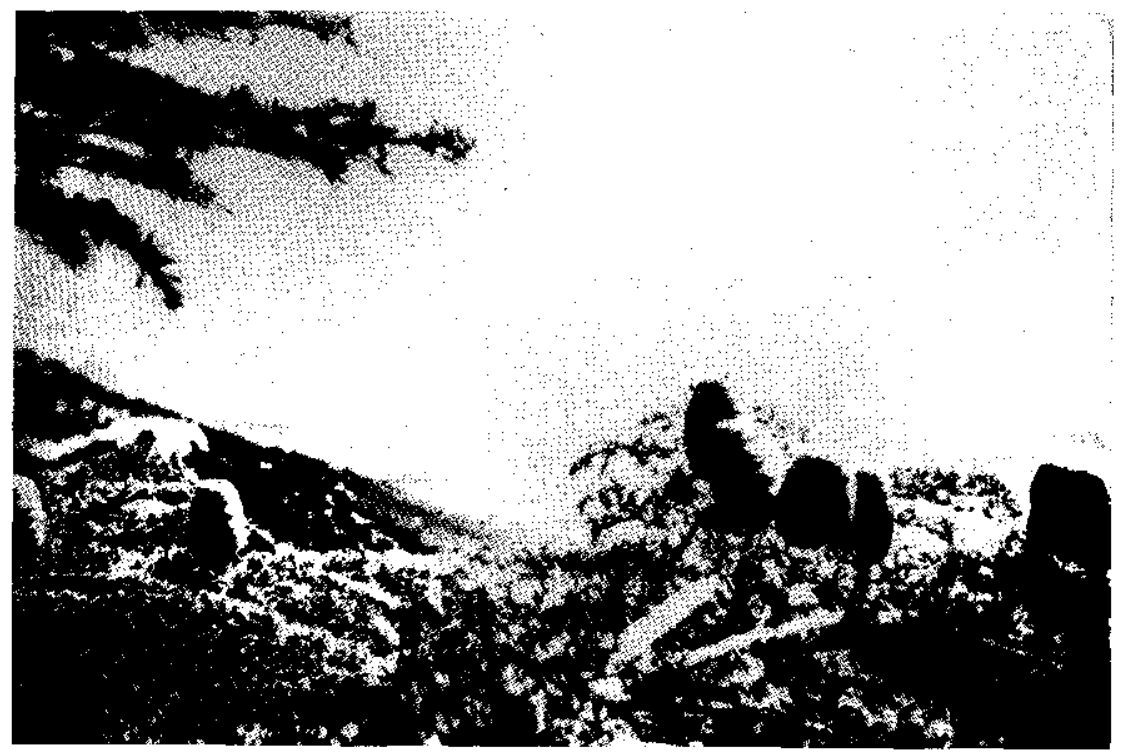

Fig. 9 - Cônes de Cedrus libani A. Rich. (Nabi Matta, Dj. Alaouite). 
L'arbre commence à fructifier assez tard, entre 35 et 40 ans, mais ce n'est qu'après 60 ans que les cônes sont abondants.

Le Cèdre du Liban est un arbre très longévif. A Bcharré, au Liban, on trouve une douzaine d'arbres auxquels on attribue plus d'un millier d'années. Les plus beaux ont 25 mètres de hauteur et 12 mètres de circonférence à la base $d u$ tronc.

Le bois du Cèdre est de couleur rouge ou blane rougeâtre et a une odeur très agréable; l'aubier est blane et bien tranché. Le bois d'automne est moins lignifié que celui du printemps. La fibre est courte et le grain est fin et susceptible d'un beau poli. Il est tendre et facile à travailler. Son goût est amer, le mettant ainsi à l'abri des rats. Il se conserve très longtemps. Pour cette raison les Anciens 1'utilisaient pour la fabrication des statues de leurs dieux et des roi et pour la construction des navires. On rappelle souvent, à propos, que les poutres du temple d'Appolon à Utique, dont parle Pline, étaient en bon état 1178 ans après sa construction.

Le bois et les cônes de Cèdre renferment une résine appelée "Cedria" que les Anciens utilisaient comme "matière protectrice ». Les Anciens Egyptiens lui donnaient le nom de "Vie des morts " et utilisaient la sciure de bois de Cèdre dans la momification. Ces sciures ont été retrouvées dans les tombeaux des Pharaons.

Le bois de Cèdre est estimé pour la construction des maisons et des ponts, la fabrication des traverses de chemin de fer, etc. Il est très peu utilisé par la caisserie à cause de l'odeur qu'il dégage.

\section{Auto6cologie du Cedrus libani dans le Djebel Alaouite}

\subsection{Altitude Et Exposimion}

Nous avons vu, dans ce qui précède, que le Cedrus libani était essentiellement montagnard et se localisait dans les parties supérieures des Montagnes du Proche Orient. Dans le Djebel Alaouite, il en est de même. En effet, à partir de $1000 \mathrm{~m}$ d'altitude et sur le versant Est du Djebel Alaouite, on commence à apercevoir quelques Cèdres mélangés le plus souvent avec : Quercus infectoria, 
Quercus calliprinos, Quercus Cerris, Pistacia palaestina, etc. Cette limite inférieure de $1000 \mathrm{~m}$ d'altitude parait basse si nous la comparons à la limite inférieure du Cedrus libani dans le Taurus, l'Amanus, d'autant plus que le Cèdre occupe le versant Est, donc sec, du Djebel Alaouite.

L'explication qui nous satisfait est celle qui consiste à imputer cette descente de la limite altitudinale, sur un versant Est, à la remontée des brouillards et leur installation à cette limite de $1000 \mathrm{~m}$ et plus haut à partir $d u$ grand marécage du Ghaab qui se trouve aux pieds du versant Est du Djebel Alaouite. Il se produit une certaine compensation de facteurs; l'humidité due au brouillard compensant la sécheresse due à une exposition Est. Le marécage du Ghaab est en voie d'assèchement par l'Etat et les remontées des brouillards ont presque disparu. Nous assisterons, probablement dans quelques dizaines d'années, à une remontée de la limite inférieure du Cèdre.

Bien que le Cèdre apparaisse à partir de $1000 \mathrm{~m}$, il ne se trouve dans son optimum écologique qu'à partir de $1200 \mathrm{~m}$. Il monte jusqu'à $1580 \mathrm{~m}$, le plus haut sommet du Djebel Alaouite.

Le Cèdre est absent sur le versant Ouest où il est remplacé par le sapin de Cilicie, plus exigeant que lui en humidité.

\subsection{Conditions chimatrques}

Le Cèdre résiste bien aux basses températures et grâce à sa ramure horizontale et flexible, il supporte bien la neige. Il préfère les versants Nord et Ouest, qui sont froids et humides. Mais comme on l'a vu plus haut, il est chassé de ces expositions par le sapin de Cilicie. La sécheresse combinée avec des températures très élevées en été et froides en hiver lui est défavorable.

Le Cèdre est à son optimum écologique dans l'étage bioclimatique méditerranéen humide et froid et dans l'étage perhumide froid. Dans ce dernier, la neige est assez fréquente en hiver et sert à alimenter les jeunes semis de Cèdres à la belle saison.

\subsection{Conditions Édaphiques}

Le Cèdre est installé dans le Djebel Alaouite sur les calcaires compacts jurassiques, crétacés et éocènes, surmontés d'argile de 
décalcification. Ce calcaire compact est très fissuré et la Terra rossa remplit ses fissures. On ne le trouve pas sur roche-mère siliceuse. Dans les autres pays, le Cèdre se trouve sur des rochesmères très variées et il est, par conséquent, indifférent à la composition chimique des sols, mais, par contre, il est sensible à la texture et à la structure des sols, surtout lors de sa régénération naturelle et pendant son jeune âge. La régénération est plus facile sur des sols meubles, perméables et de bonne structure et permettant une meilleure alimentation en eau du jeune semis.

On voit souvent beaucoup de jeunes semis dans le Nabi Matta sur le calcaire compact au printemps, mais rares sont ceux qui résistent à la sécheresse de l'été. Mais sous les chênes, où existe un horizon $A_{1}$ humifère et de bonne structure facilitant une meilleure alimentation en eau, les semis sont relativement plus nombreux. De plus, les chênes protègent les jeunes semis contre l'insolation. Le dégagement ultérieur de ces semis est nécessaire.

La régénération naturelle était plus aisée pour le Cèdre avant l'assèchement du Ghaab. La sécheresse excessive de l'été de notre pays sera un des facteurs les plus redoutables pour la régénération des cédraies.

Une fois installé, le jeune Cèdre se développe bien et s'accroche bien au sol. Grâce à leur système radiculaire, les arbres adultes peuvent utiliser l'eau emmagasinée dans les fissures du calcaire compact. Bien que superficiels, ces sols formés sur calcaire compact, fissuré, ne sont pas défavorables au cèdre.

\subsection{TEMPERAMENT}

Le Cèdre supporte très longtemps le couvert et pent rester de nombreuses années étouffé par les chênes pour repartir vigoureusement dès qu'il est dégagé, après avoir généralement bien supporté la crise d'isolement ; de même à l'état de gaulis, il demeure, longtemps, comme le sapin de Cilicie sous le couvert des vieux chênes.

D'un autre côté, on a remarqué qu'il se régénérait assez facilement dans les endroits découverts, sur les lisières, dans les vides à condition qu'il y ait un certain abri latéral. 
Le Cèdre du Liban est donc une essence de demi-lumière. Nous pouvons profiter de ce tempérament pour reconstituer la cédraie là où elle a disparu et a été remplacée par une végétation de feuillus divers.

\section{Les peuplements de Cèdres dans le Djebel Alaouite}

Par suite de l'action de l'homme qui, durant des millénaires, a abusé de l'exploitation des forêts de Cèdres, même jusqu'à une date très récente, un désordre règne dans la zone où vit le cèdre, dans le Djebel Alaouite. Les coupes abusives, non contrôlées et découvrant fréquemment le sol, ont défavorisé le cèdre, essence qui nécessite un certain abri pendant son jeune âge, spécialement dans des stations sur calcaire compact et dans un pays où l'été est très sec, au profit des essences de lumière, comme les chênes. L'association du Cedrus libani (Cedretum), au sens strict $\mathrm{du}$ mot, n'existe pas dans le Djebel Alaonite. Ce qu'on trouve est, en réalité, un mélange d'espèces arborescentes très variées et appartenant à des groupements variés au milieu desquelles le cèdre se trouve disséminé, par pieds isolés et, quelques fois, par petits bouquets.

Il n'y a aucun doute que, potentiellement, l'étage situé entre 1200 et $1500 \mathrm{~m}$ sur le versant Est est celui du Cedretum, mais pour des raisons anthropogènes antérieures, cette association ne se manifeste plus à présent.

Parmi les espèces arborescentes qui vivent avec le cèdre, nous citerons :

\section{Quercus Cerris}

Q. Brantii

Q. Libani

Q. infectoria

Q. calliprinos

Q. Ehrenbergii (très rare)

Acer monspessulanum

Acer hyrcanum

Sorbus torminalis

Sorbus umbellata var. flabellifolia

Prunus ursina

\author{
Styrax officinale \\ Pyrus syriaca \\ Cotoneaster nummularia \\ Ostrya carpinifolia \\ Carpinus orientalis \\ Pistacia palaestina \\ Laurus nobilis \\ Juniperus drupacea \\ J. Oxycedrus \\ Fraxinus ornus
}


Parmi les autres espèces qui accompagnent le cèdre on trouve : Hedera Helix, Paeonia corallina, Helichrysum plicatum, Orchis comperiana, Origanum amanum, Rubia Aucheri, Aspidium Filix-Mas, etc.

La reconstitution du Cedretum n'est pas facile mais elle n'est pas non plus impossible. Le forestier peut profiter de l'abri créé par les espèces arborescentes pour encourager la régénération naturelle ou faire des semis artificiels de cèdre sous les chênes en faisant des coupes d'abri appropriées. Des plantations de jeunes cèdres peuvent être envisagées sous ces chênes et qu'on dégagera ultérieurement. Mais il est de toute importance d'interdire d'une façon très rigoureuse le pâturage.

Le Cèdre, cet arbre qui etait lié à l'histoire des plus hautes civilisations du Proche-Orient, et qui a rendu tant de services à l'homme, mérite d'être protégé par lui pour empêcher sa disparition des régions où il était toujours l'emblême.

\section{Les forêts de Còdres de Syrie et du Liban dans l'histoire}

Au début de la période historique, les forêts de cèdres convraient de très vastes étendues dans les montagnes de Syrie et du Liban. Le cèdre, arbre très apprécié par les peuples des anciennes civilisations du Proche-Orient, était appelé par ceux-ci le "Roi de la forêt ".

Les Phéniciens appréciaient beaucoup le bois de cèdre, incorruptible et résistant à l'eau de mer, et l'ont beaucoup utilisé pour la fabrication de leurs navires et l'élargissement de leur commerce sur le pourtour de la Méditerranée. Installés sur la côte de Syrie et du Liban, les Phéniciens avaient à la portée de leurs mains les forêts de cèdres qui couvraient le versant Ouest. Ce même peuple a utilisé le bois de Cèdre pour la construction des temples, pour la sculpture et la fabrication de chaises, de verres, d'assiettes, etc.

Les forêts de cèdres étaient, pour les Phéniciens une autre source de richesse. Ils faisaient le commerce du bois de cèdre avec l'Egypte, pays dépourvu de forêts.

Plusieurs milliers d'années avant l'ère chrétienne, les Egyp- 
tiens tiraient de ces forêts tout le bois d'oeuvre qui leur était nécessaire pour la construction des temples et des sarcophages et dont on retrouve encore tant d'exemplaires intacts pans les beaux sarcophages ornés de peinture. Les Pharaons, lors de leurs luttes avec les rois syriens, ne manquaient jamais de leur imposer un lourd tribut de bois de cèdre.

L'Histoire nous raconte que le Roi David a demandé aux. artistes phéniciens, au dixième siècle avant Jésus-Christ, de lui construire un certain nombre de châteaux et maisons en bois de cèdre. Les artistes phéniciens ont également construit à Salomon un grand château en bois de cèdre et qui a reçu le nom de " château de la forêt du Liban".

Les Assyriens ont également utilisé le bois de cèdre pour divers usages. A l'époque romaine, les forêts de cèdres subsistaient encore bien qu'appauvries. On cite souvent que la croix de JésusChrist était en cèdre.

En un mot, les cédraies de Syrie et du Liban ont, durant des millénaires, alimenté les premières civilisations de la vallée du Nil et du Proche-Orient.

\section{III - ABIES CLLICICA (Ant. et Ky.) De Tehilhateheff (1854).}

Nom local arabe: Al Ohouhh

\section{Aire géographique de l'Abies eilicica}

Le genre Abies est très ancien. Il est connu avec certitude dès le Miocène. De l'avis de Gausssen (1952), les genres Abies et Cedrus ont un ancêtre commun lointain; mais le genre Abies s'est éloigné du caractère de cet ancêtre beaucoup plus que le genre Cedrus. Le sapin de Cilicie fait partie d'un groupe d'espèces voisines occupant dans les montagnes du pourtour de la Méditerranée une série d'aires disjointes souvent de peu d'importance.

Il semble que toutes ces espèces aient un ancêtre commun tertiaire, et que l'isolement géographique causé par l'effondrement de la Méditerranée ait disloqué l'aire géographique de cet ancêtre 
en plusieurs petites aires favorisant ainsi l'apparition de plusieurs espèces endémiques, mais très proches parentes entre elles.

Les principales de ces espèces sont :

Abies Pinsapo Boiss. (1838) - Sapin d'Espagne : il habite entre 1000 et $1500 \mathrm{~m}$ la Sierra Nevada, les montagnes de Ronda, las Nieves où il forme des peuplements de très faible importance.

Abies Marocana Trabut: cette espèce semble n'être qu'une forme de l'Abies Pinsapo habitant les montagnes calcaires de Chechaouen au Maroc, entre 1650 et $2150 \mathrm{~m}$ d'altitude.

Abies numidica de Lannoy (1866) (A. barborensis Letourneux [1888]) : ce sapin est endémique en Kabylie (Algérie) sur le Babor et le Tababor, entre 1600 et $2000 \mathrm{~m}$ d'altitude. Son aire est très réduite et ne dépasse pas quelques centaines d'hectares.

Abies cephalonica Loud. (1838) : il habite entre 800 et $1600 \mathrm{~m}$ d'altitude les montagnes de la Grèce et des îles Ioniennes.

Abies cilicica (Ant. et $\mathrm{Ky}$.) De Tchihatcheff (1854) : il habite entre 1200 et $2000 \mathrm{~m}$ les montagnes du Proche-Orient.

Abies Nordmanniana Lk. (1841) - Sapin du Caucase : il habite les montagnes du Nord de l'Asie Mineure et les ramifications occidentales du Caucase.

Toutes ces espèces présentent des caractères morphologiques et biologiques très nets, mais elles s'hybrident aisément entre elles et avec l'Abies alba Mill. (PourTer 1951).

On connait beaucoup de formes géographiques de ces sapins et d'assez nombreuses variétés différant du type par le port, les dimensions des feuilles, ou leur coloration, ainsi que de nombreux hybrides. Jusq'à présent on ne connait aucune variété de l'Abies cicilica et aucun hybride.

D'autres sapins méditerranéens endémiques existant dans certaines montagnes du pourtour de la Méditerranée, mais n'ayant d'intérêt qu'au point de vue botanique, assurent la transition entre ces six types principaux, comme: A. nebrodensis (Lojac.) Mattei des montagnes de Sicile; A. Borisii-regis Mattfeld du N.W. de la Grèce.

Toutes ces espèces sont d'autant plus semblables que leurs aires sont plus voisines.

L'Abies cilicica est confiné dans les parties élevées des montagnes du Proche-Orient. Il est inexistant à Chypre et en Palestine. On le trouve dans le Taurus, en Cilicie, l'Amanus, le Djebel Alaouite et le Liban.

Dans l'Amanus, il habite la zone montagneuse comprise entre 
1900 et $2100 \mathrm{~m}$ d'altitude, Dans cette même zone on rencontre le Cedrus libani et le Fragus silvatica var. orientalis.

Dans le Djebel Alaouite, on le trouve sur les versants Ouest et Nord-Ouest (frais et humides) entre 1200 et $1500 \mathrm{~m}$ d'altitude. Dans le Liban, on le trouve sur le versant occidental de la châine du Djebel Qamoua, au-dessus de Bä̈nou et de Fnaïdiq entre 1500 et $1800 \mathrm{~m}$ d'altitude.

\section{Caractères botaniques et dendrologiques}

Le sapin de Cilicie est un grand arbre pouvant atteindre 30 à 35 mètres de hauteur. Dans la Montagne des Alaouites, il ne dépasse pas 20 à 25 mètres de haut à cause du manque de profondeur des sols sur lesquels il pousse. Son fût est droit et élancé. Son écorce est gerȩurée, écailleuse, d'un gris cendré. La cime est un cône pointu devenant tabulaire chez les sujets âgés.

Les branches sont étagées en verticilles. Les rameaux sont ordinairement distiques; les jeunes sont d'un brun olivâtre ou jaunâtre brillant, devenant très rapidement glabres. Les écailles des bourgeons sont un peu saillantes.

Les feuilles sont persistantes, planes, linéaires, éparses, parfois fasciculées au sommet des rameaux, longues de 2 à $3 \mathrm{~cm}$, larges de 1,5 à 2,5 millimètres, arrondies et parfois légèrement échancrées au sommet, d'un vert pâle en dessus, glauques et carenées en dessous avec 2 lignes blanches de stomates parallèles à la carène, laissant après leur chute une cicatrice arrondie.

Les fleurs sont unisexáes, monoiques et en forme de châtons. Les mâles, jaunes-verdâtres sont axillaires et formées de bractées écailleuses formant un involucre à la base et d'étamines portant chacune deux sacs polliniques. Les femelles, d'un vert jaunâtre, solitaires, dressées, naissant à l'extrêmité des rameaux qui ne se développent pas et, par suite, paraissant latérales, sont formées de feuilles carpellaires nombreuses dont chacune est doublée à sa base, extérieurement, d'une bractée libre et plus ou moins développée, et porte sur sa face interne deux ovules réfléchis.

Les cônes sont dressés, très grands, de $20-25 \mathrm{~cm}$ de long sur 5 de large, d'un brun rougeâtre. Les écailles, disposées en spirales 
autour d'un axe appelê rachis, sont brusquement retrécies en onglet à leur base, beaucoup plus larges que longues, un peu tomenteuses sur les bords, ceux-ci se prolongeant de chaque côté en une pointe crochue. Les bractées se détachent à maturité du rachis du cône qui persiste sur l'arbre. Chaque écaille fertile porte deux graines largement ailées de $1 \mathrm{~cm}$ de long, an bas de sa face interne.

Le système radiculaire est puissant.

Le bois, est léger, tendre, et susceptible d'emplois courants. Ce n'est pas un bois de haute qualité, mais en compensation son fût est droit et cylindrique. Il est, en outre, recherché pour la pâte à papier, mais étant donné l'état de nos forêts de sapin et les faibles superficies qu'elles couvrent actuellement, ce débouché n'existe pas en Syrie.

Le sapin de Cilicie est un arbre très décoratif. Les jeunes sujets sont recherchés pour la fête de Noël.

\section{Autócologie de l'Abies cilicica dans le Djebel Alaouite}

\subsection{Altitude ET EXPOSITION}

L'Abies cilicica est essentiellement montagnard. Dans le Djebel Alaouite, il occupe les versants ouest, nord et nord-ouest, c'est-àdire les versants frais et humides. On ne le rencontre pas à une altitude inférieure à $1150-1200 \mathrm{~m}$. C'est après Slenfé, dans le Djebel Qamoua et anquel on donne le nom de Djebel Al Chouhh, qu'il forme les peuplements les plus denses.

\subsection{Conditions climatiques}

Le sapin de Cilicie résiste bien aux basses températures. Il habite, en effet, la zone la plus froide du nord-ouest de la Syrie. La neige y est fréquente pendant l'hiver. Au printemps, toute la neige fond et contribue ainsi à augmenter les réserves hydriques des sols. La pluviométrie moyenne annuelle est très élevée et varie entre 1200 et $1300 \mathrm{~mm}$. La station météorologique la plus 
proche (Slenfé), qui est à $1000 \mathrm{~m}$ d'altitude, indique une pluviométrie de $1200 \mathrm{~mm}$ par an. La moyenne des maxima du mois le plus chaud (M) est 26,2 et la moyenne des minima du mois le plus froid (m) est 1,6. Le quotient pluviométrique d'EMBERGER (Q) est 149,5. L'optimum écologique du sapin de Cilicie chez nous est done réalisé dans l'étage bioclimatique perhumide froid.

Malgré une pluviosité annuelle assez élevée, le sapin traverse une saison séche très critique, mais il est habitué à ce rythme climatique et c'est pour cette raison qu'il est connu comme une espèce relativement résistante à la sécheresse; peut être moins rësistante que l'Abies Pinsapo, mais nettement plus résistante que l'Abies alba.

\subsection{CONDITIONS ÉDAPHIQUES}

On trouve le sapin de Cilicie dans le Djebel Alaouite sur le calcaire dolomitique alternant avec de la marne dolomitique du Crétacé moyen et sur les calcaires compacts jurassiques, crétacés et écènes recouverts d'argile de décalcification.

Ces calcaires sont profondément fissurés. Le calcaire dolomitique est plus favorable que le calcaire compact; il est plus perméable et l'eau y est mieux répartie. L'exigence du sapin de Cilicie en humidité le guide dans le choix des versants humides et frais et le choix des roches mères retenant mieux l'eau. C'est pour cette raison qu'il est totalement absent du versant Est, où il est remplacé par le cèdre qui est plus xérophile et plus rustique. que lui.

\subsection{TEMPÉRAMENT}

Le sapin de Cilicie est une essence de demi-ombre. Pendant son. jeune âge il est très sensible à l'insolation, beaucoup plus que le cèdre. Il ne se régénère naturellement bien que sous un couvert. demi-dense. Il supporte très bien et très longtemps le couvert de ses parents pour repartir vigoureusement dès qu'il est dégagé. A l'état de gaulis, il peut demeurer longtemps sous un couvert assez dense. 
Nous avons profité de ce tempérament pour essayer de reconstituer artificiellement la sapinière d'où elle a disparu et qui est actuellement remplacée par des essences de lumière de faible valeur économique : Ostrya carpinifolia, Carpinus orientalis, Quercus Libani, etc., en plantant des jeunes sapins de 4 à 5 ans sous coupe d'abri. Les jeunes sapins seront dégagés graduellement par réduction graduelle du couvert.

\section{Les peuplements d'Abies eiliciea dans le Djebel Alaouite}

L'association de l'Abies cilicica (Abietum cilicicae) au sens strict n'existe pas dans le Djebel Alaouite, comme c'est le cas pour le Cedretum. En effet, les peuplements de sapin de Cilicie qui existent dans le Djebel Al Chouhh sont surexploités depuis des millénaires. Les coupes abusives, non contrôlées, découvrant fréquemment le sol, sont défavorables pour une essence de demiombre nécessitant un certain abri pour se régénérer. L'abri est d'autant plus nécessaire que nous sommes dans une région très sèche et très chaude pendant l'été. Le résultat est le remplacement du sapin par des essences héliophiles comme: Quercus Libani, Q. brantii, Q. Cerris, etc. appartenant à des associations variées. En outre, ces essences pouvant se perpétuer par rejets de souche, le problème de leur régénération naturelle par voie de semis ne se pose pas.

L'homme a, par conséquent, complètement bouleversé la nature dans cette région en remplaçant l'association climacique qui est l'Abietum cilicicae par un autre gronpement non défini. Grâce à la protection de ces forêts à l'époque actuelle, nous assistons à une réinstallation du sapin sous les chênes.

Parmi les espèces arborescentes accompagnant l'Abies cilicica nous citerons :

\author{
Quercus Cerris \\ Q. Brantii \\ Q. Libani \\ Q. infectoria \\ Ostrya carpinifolia \\ Carpinus orientalis \\ Acer hyrcanum \\ Staphylea pinnata \\ Styrax officinale \\ Cerasus Mahaleb
}

Sorbus torminalis

S. umbellata var. flabellifolia

Laurus nobilis

Juniperus drupacea

J. Oxycedrus

Acer monspessulanum

Fraxinus ornus

Daphne oleoides

Cornus sanguinea ssp. australis

Lonicera orientalis 
D'autres espèces accompagnent le sapin aussi : Hedera helix, Rubia Aucheri, Aspidium Filix-Mas, Helichrysum plicatum, Paвonia corallina, etc.

Beaucoup d'espèces septentrionales (Sorbus torminalis, Sorbus umbellata, etc.) vivent dans l'étage du sapin et y constituent des enclaves au milieu d'une végétation typiquement méditerranéenne.

Chapitre cinquik̀me

LA GARRIGUE A QUERCUS CALLIPRINOS WEBB ET PISTACIA PALAESTINA BOISS. DU DJEBEL ALAOUTTE (Pistacieto-Quercetum calliprini Alaouitaea Nahal)

\section{Introduction}

La garrigue est la formation végétale arborescente la plus répandue en Syrie. Elle est également, la formation la plus commune et la plus caractéristique de la Méditerranée Orientale. L'association "Quereus calliprinos et Pistacia palaestina" (Pistacieto-Quercetum calliprini) est la forme la plus répandue de la garrigue orientale et peut être considérée comme représentant le paysage caractéristique des régions boisées de Syrie.

Une autre association, le Poterietum spinosi, qui provient de sa dégradation, comme on le verra plus loin, est également caractéristique de la Méditerranée Orientale et y occupe des superficies assez importantes.

Dans le Nord-Ouest de la Syrie et, plus particulièrement dans la Montagne des Alaouites, cette garrigue couvre de grandes surfaces. Elle a toujours été d'une utilité capitale pour les habitants de cette montagne en leur permettant de satisfaire leurs besoins en bois de chauffage et bois de construction et en leur assurant la nourriture de leur bétail, presque exclusivement constitué de chèvres, la litière de leurs troupeaux, la fertilisation de leur récolte, etc. 
L'homme a, par conséquent, fait disparaître la végétation primitive et l'a remplacée par une végétation secondaire, plus riche en espèces ligneuses et convenant plus particulièrement à ses besoins. Mais, malheuresement, la dégradation ne s'arrêtait pas à ce stade et, souvent, quand les dents de la chèvre s'associaient à la hache du paysan, de nouvelles formations végétales, beaucoup moins intéressantes et protégeant moins le sol, apparaissaient et soumettaient ainsi ce dernier à l'action de l'érosion sous ses multiples formes. Dans les cas extrêmes, qui hélas ne sont pas rares dans notre région, un désert blanc rocheux remplace les forêts primitives. Par imprudence, et sans se préoccuper de l'avenir, l'homme a done provoqué dans certains endroits la destruction de son propre patrimoine, de sa propre ressource de vie: le sol.

L'étude de la garrigue à Querous calliprinos et Pistacia palaestina paraît donc importante aussi bien du point de vue historique que scientifique et économique, car, liée à la vie des populations villageoises dans les siècles derniers, elle ne présente plus actuellement qu'un intérêt très faible pour eux. Par ailleurs, la connaissance des phases de dégradation de la forêt primitive ne peut être que très utile aux forestiers et écologistes qui envisagent la mise en valeur de cette région.

Nous envisageons, dans cet article, l'étude de la garrigue à Quercus calliprinos et Pistacia palaestina du point de vue floristique, écologique et phytodynamique en essayant d'en dégager les renseignements écologiques pouvant être utiles aux forestiers.

Avant de commencer l'étude de la garrigue, nous tenons à définir le terme garrigue et faire connaissance avec les deux espèces les plus importantes qui la composent, c'est-à-dire, le Quercus calliprinos et le Pistacia palaestina.

\section{Definition du terme "garrigue "}

Nous appelons " garrigue " une formation végétale arborescente, xérophile, peu développée en hauteur, plus ou moins ouverte, installée le plus souvent sur substratum calcaire et tirant son origine de la destruction de la forêt primitive. Elle peut également représenter une étape assez avancée de la réoccupation du sol 
nu par la végétation spontanée dans le sens de l'évolution progressive vers le climax.

Nous tenons à préciser ici que la garrigue peut être très fermée dans la région que nous étudions.

\section{Quereus calliprinos Webb et Pistacia palaestina Boiss.}

\subsection{Quercus calliprinos Webb (nom local arabe : Al Sindyâne)}

Le Quercus calliprinos Webb (chêne kermès) est l'espèce vicariante du Quercus coccifera $\mathrm{L}$. de Ia Méditerranée occidentale et de l'Afrique du Nord. De nombreux botanistes ont confondu cette espèce de la Méditerranée orientale avec le Quercus coccifera L. de la Méditerranée occidentale. C'était l'attitude de BorssLer 1879 (Flora Orientalis, vol. IV, p. 1169, 1879) qui a subdivisé le chêne kermès oriental en plusieurs variétés : Quereus coccifera $\mathbf{L}$. var. genuina, integrifolia, calliprinos, pseudococcifera, palaestina, rigida; de Bouloumoy 1930 (Flore du Liban et de la Syrie, p. 314, 1930); de Post 1932 (F'lora of Syria, Palestine and Sinai, 1932); Thí́вaUT 1953 (Flore Libano-syrienne, 1ère partie, 1953).

Nous avons suivi cette voie également dans nos études antérieures. Mais notre visite de la garrigue à Quercus coccifera de la France Méditerranéenne nous a permis de déceler des différences morphologiques assez importantes entre le chêne kermès qui forme l'élément principal de cette garrigue et celui de la garrigue de la Méditerranée orientale. Une différence essentielle, qui est de première importance pour les forestier, tient a la différence de taille entre les 2 chênes. Le chêne Kermès occidental est un arbrisseau très bas, s'étalant au-dessus du sol alors que le chêne oriental est un petit arbre pouvant atteindre 10 ̀े 15 mètres de hant dans des conditions écologiques très voisines.

De très beaux spécimens sont obsérvés autour des lieux saints (Mazar) dans la montagne des Alaouites. D'autres caractères morphologiques des feuilles et des cupules différencient également ces deux chênes comme on le verra dans le paragraphe suivant.

WEBB (1838) est le premier botaniste qui a donné une valeur spécifique au chêne kermès oriental et l'a appelé Quercus calli- 
prinos alors que les autres botanistes qui sont venus après lui (Borssier, Bouloumoy, Post, Thiebaut, etc.) l'ont réduit à une variété de Quercus coccifera $\mathrm{L}$, ou l'on confondu avec lui.

Nous sommes de l'avis de WEBB pour faire du chêne kermès oriental une espèce distincte. C'était également l'avis de DE CANdolle, Eig (1933), A. Camus (1933). Ce dernier botaniste a distingué dans le Quercus calliprinos Webb plusieurs variétés suivant la structure et la forme de la cupule (var. eigii Camus; var. Fenzlii (Ky.) A. Camus ; var. eucalliprinos DC.; var. inops DC. ; var. valida DC.; var. leptolepis DC.; var. arcuata DC. [Q. palaestina Ky.]) (Bull. Soc. Bot. Fr., p. 355, 1933). Il est important de signaler que toutes ces variétés ont les mêmes exigences écologiques et se rencontrent presque souvent ensemble et montrent des formes de transition.

Quoiqu'il en soit, le Quercus coccifera L. et le Quercus calliprinos Webb restent deux espèces vicariantes dont la première constitue l'élément principal de la garrigue de la Méditerranée occidentale et la seconde, l'élément principal de la garrigue de la Méditerranée orientale.

\subsubsection{Caractères botaniques et dendrologiques}

Le Quercus calliprinos Webb appartient à la famille des $\mathrm{Cu}$ pulifères (ou des Fagacées), au genre Quercus, au sous-genre Euquercus Hicke et A. Camus, à la section Cerris Spach, à la sous-section cocciferae Schitt.

C'est un petit arbre pouvant atteindre $15 \mathrm{~m}$ de hant, à tronc peu droit, pouvant atteindre $80-90 \mathrm{~cm}$ de diamètre, à rameaux robustes. Les bourgeons sont très rapprochés au sommet des rameaux, petits, ovoïdes, plus ou moins tomenteux ordinairement, brun rougeâtre ou grisâtre, munis d'écailles arrondies poilues et ciliées.

La cime est globuleuse, étalée, et assez dense. Les feuilles sont persistantes, planes, oblongues à lancéolées, au sommet arrondies, obtuses ou subaigues, de taille assez variable, longues 2,5-5 cm, larges de 1-2 cm, très coriaces, concolores, les jeunes munies de poils épars, rapidement glabre sur les deux faces, à bords serrés-mucronés, très rarement épineux-dentés et à dents 
très dressées, ou plus rarement à bord entier ; pétiole long de 2 à $5 \mathrm{~mm}$ densément pubescent, à tomentum assez persistant.

Les fleurs sont monoïques; les mâles en châtons grêles, axillaires ou rapprochées au sommet des rameaux de 5 à $6 \mathrm{~mm}$ de

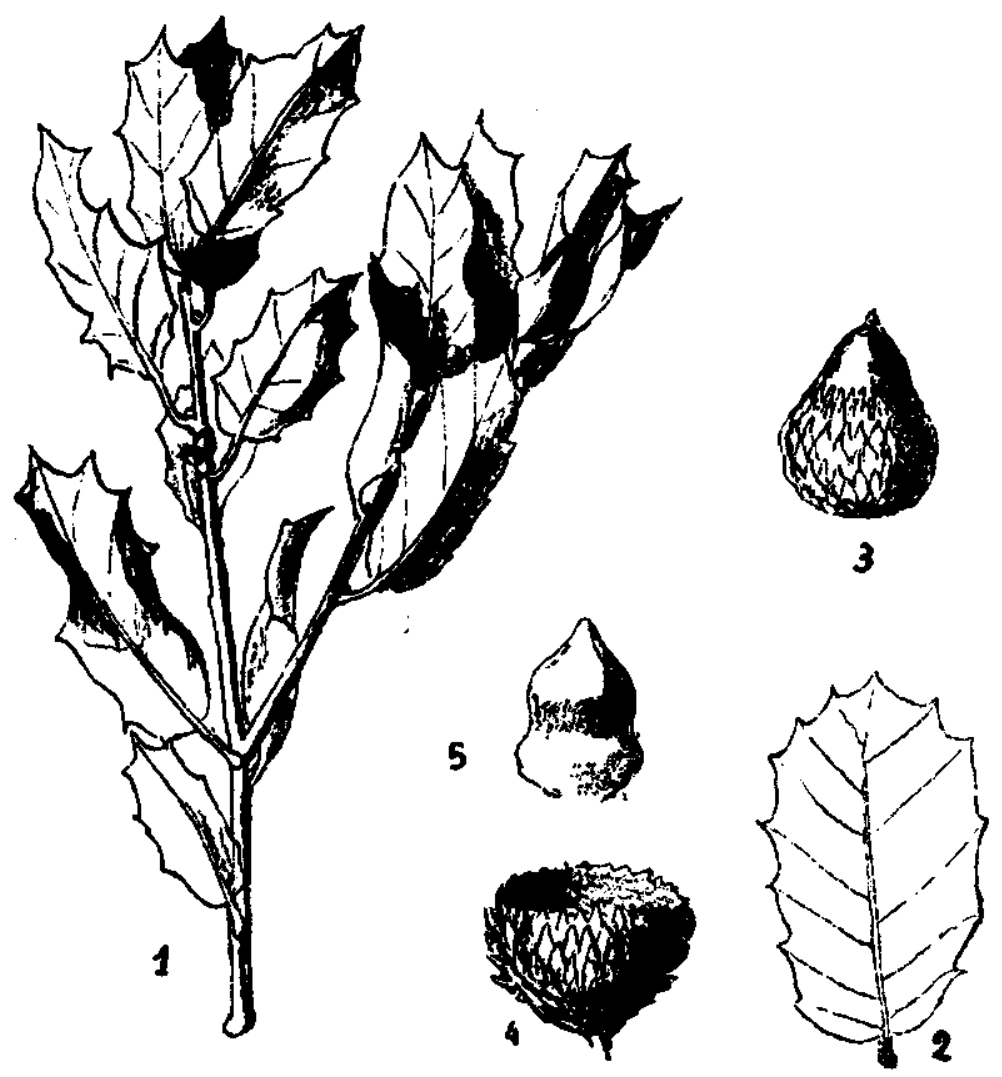

Fig. 10 - Quercus cocoifera L. - 1 : rameau feuillé. 2 : fenille, face inférieure de la varieté imbricata d'Andalousie. 3 : cupule et fruit. 4 : cupule. 5 : écaille moyenne très grossie de cette cupule.

diamètre; axe poilu; bractées étroites, pubescentes; étamines 4-5; les femelles longues de 1 à $2 \mathrm{~cm}$, ̀̀ axe très tomenteux.

Le fruit (gland) est indéhiscent, monosperme, entouré d'une cupule à sa base. Les cupules sont isolées ou géminées, hémisphériques ou subcampanulées, grandes, épaisses, ligneuses, couvrant 
presque tout le fruit, on les $2 / 3$ on les $3 / 4$ du fruit, soyeuses en dedans, couvertes d'écailles nombreuses ; munies de poils soyeux, blanchâtres, parfois brunâtres à l'extrêmité, parfois toutes dressées, imbriquées, apprimées, ovales, obtuses, courtes, les moyennes plus allongées, lancéolées, à partie libre atteignant parfois 4 à $5 \mathrm{~mm}$, ordinariement étalées ou réfléchies, piquantes au sommet, les supérieures linéaires, lancéolées, dressées, étalées ou réfléchies.

Le gland est inclus ou peu exsert, rarement très exsert, brièvement arrondi, rarement allongé, obtus et mucroné au sommet, haut de 1,5-3,5 cm de 1,2-1,8 cm de diamètre.

Le bois est dur et utilisé surtout pour le chanffage. L'écorce, surtout celle des racines, renferme du tannin.

Le Quercus calliprinos est un arbre à développement lent et qui vit très longtemps. On trouve au Proche-Orient des individus âgés de 700 ans.

Nous résumons dans ce qui suit, les principaux caractères permettant de différencier le $Q$. calliprinos du $Q$. coccifera :

Quercus calliprinos Webb

1. Arbre pouvant atteindre $15 \mathrm{~m}$

2. Feuilles généralement oblongues lancéolées, rarement ovales

3. Bords des feuilles peu épais serrésmucronés, très rarement épineuxdentés et à dents dressées

4. Rameaux à tomentum très dense

5. Ecailles des bourgeons poilues

6. Axe des châtons femelles tomenteux

7. Nombre des stigmates de 3 à 4

8. Ecailles des cupules longues de 4-10 mm, tomenteuses ; pointe des écailles assez longue, non épineuse

9. Gland le plus souvent plus ou moins arrondi au sommet
Quereus coccifera L.

1. Arbrisseau ne dépassant pas 4.5 m

2. Feuilles généralement ovales ou élliptiques, rarement oblongues

3. Bords des feuilles épais, ondulé, ordinairement a 4 ou 6 paires de dents épineuses, divariquées, étalées, aculéolées

4. Rameaux très peu tomenteux et rapidement glabre

5 Ecailles des bourgeons généralement glabres

6. Axe des châtons femelles glabres. cent ou glabre

7. Nombre des stigmates de 5 a 6

8. Ecailles des cupules plus courtes, de 1,5 a $3 \mathrm{~mm}$, aigues, raides; pointe des écailles courte, épineuse, presque vulnérante

9. Gland le plus souvent ovoide.

Le Quercus calliprinos présente une grande variabilité qui se manifeste aussi bien dans le fruit que dans les feuilles, mais la 

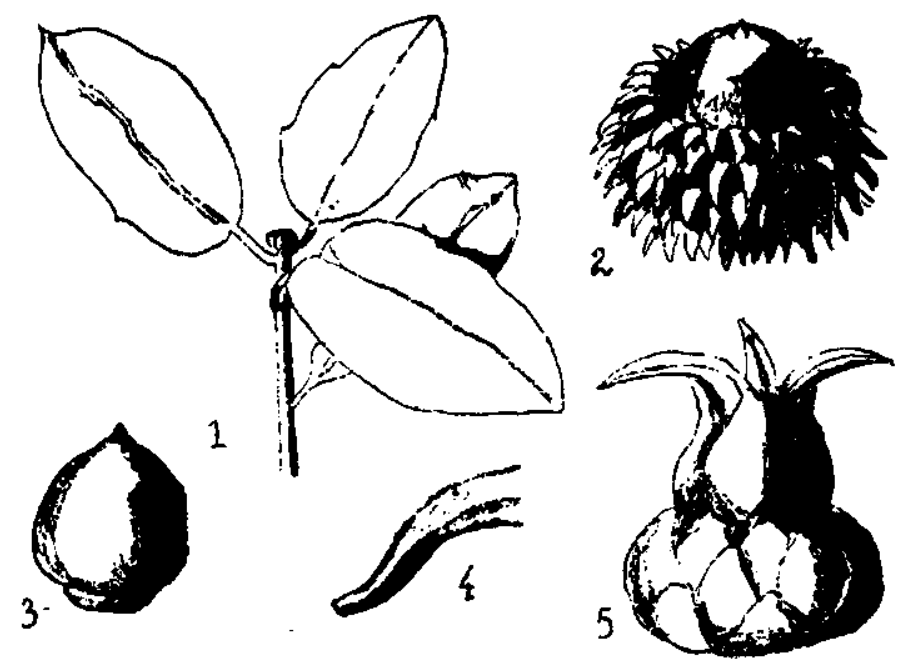

Fig. 11 - Q. calliprinos Webb var. eucalliprinos DC. - 1 : ramean feuillé. 2) Toupule et fruit. $3:$ gland. 4 : écaille très grossie. 5 : très jeune cupule, périanthe et styles très grossis.

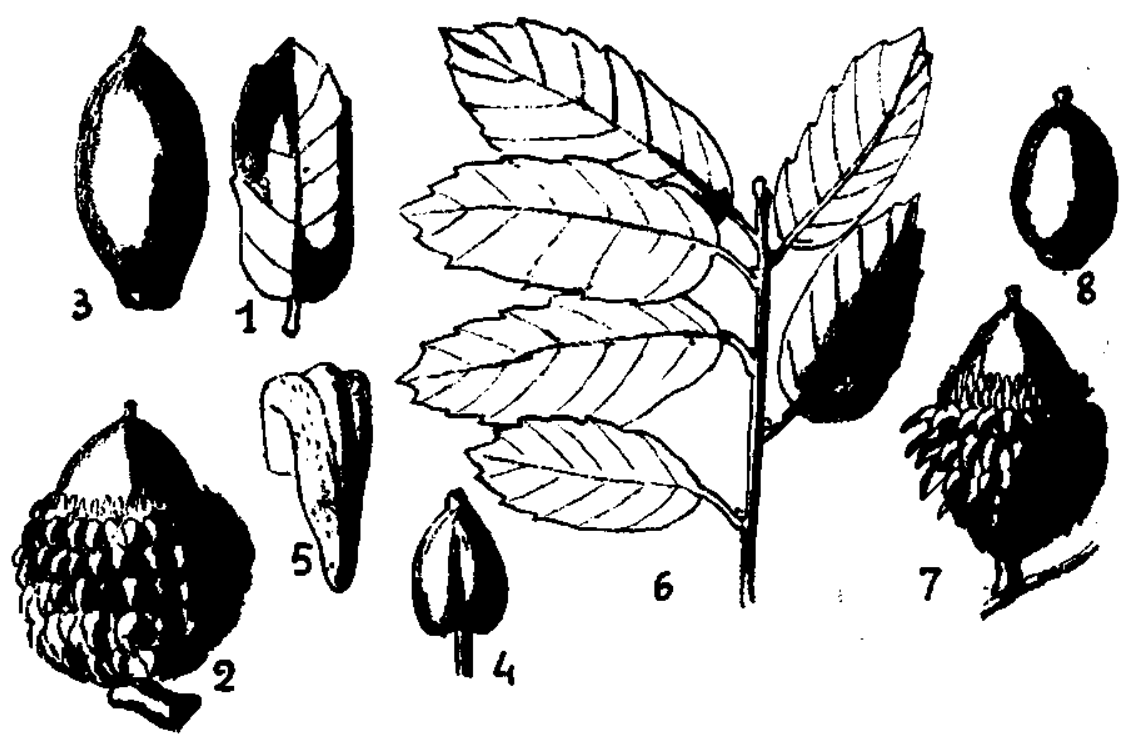

Fig. 12 - Q. calliprinos var. areuata DC. - 1 : feuille face supérieure. 2 : cupule et fruit. 3 : gland. 4 : anthère très grossie. 5 : écaille de la cupule très grossie. 6 : rameau feuillé. 7 : fruit et cupule. 8: fruit. 
variabilité du fruit est plus constante et mérite d'être retenue plus que celle des fenilles.

A. CAMUs (Monographie du genre Quercus, 1936-1938) distingue plusieurs variétés de Quercus calliprinos suivant la forme, la dimension et la structure de la cupule et dont nous citerons les plus répandues.

\section{1 - Ecailles de la cupule étalées ou recourbées}

var. eucalliprinos DC. 1864 (Q. psendococcifera Labill., 1798-1800)

Cupule munie d'écailles serrées, les médianes de 5 a $6 \mathrm{~mm}$; lancéolées, ordinairement étalées; gland épais, inclus ou peu exsert ; écailles à bord très peu ou pas dentées.

Cette variété est assez répandue dans notre région.

var. dispar Ky. 1866 exsert.

Cette variété se distingue de la précédente par son fruit plus long et plus

var. valida DC. 1864 (Q. valida Ky.)

Cupule atteignant $3 \mathrm{~cm}$ de hauteur et $4 \mathrm{~cm}$ de diamètre environ, munie d'écailles, les inférieures courtes, ovales, robustes, les médianes inférieures allongées, étalées, les supérieures allongées, atteignant $1 \mathrm{~cm}$, d'abord dressées, puis souvent oncinées-recurvées, gland mûr égalant la cupule ou la dépassant, épais, obtus, plus velu au sommet, que dans les autres variétés.

var. arcuata DC. 1864 (Q. palaestina $\mathrm{K}_{\mathrm{y}}$; ; . coccifera var. palaestina Boisss.; Q. calliprinos var. palaestina (Ky.) Zoh.).

Feuilles planes ou presque, à peine denticulées, atteignant 3 à $4 \mathrm{~cm}$ de longueur ; pédoncule fructifère portant souvent deux cupules ; cupule campa. nulée ou subhémisphérique, haute de 1,5 à $2,5 \mathrm{~cm}$, de 1,8 à $2,7 \mathrm{~cm}$ de diamètre, couvrant les $2 / 3$ ou les $3 / 4$ du gland, à écailles basilaires, peu nombreuses, ovales, obtuses, les autres étroitement lancéolées, très recourbées en dehors, sauf les supérieures ordinairement allongées et dressées ; gland ovoide, long de 3-5 cm, nettement mucroné au sommet; cicatrice assez convexe.

Cette variété existe dans notre région. C'est le chêne le plus grand de ce groupe. Il existe un spécimen de chểe, appelé le chêne d'Abraham, près d'Hebron, sous le couvent russe en Palestine ; son trone a plus de $7 \mathrm{~m}$ de circonférence.

var. Eigii A. Camus 1933

Cupule blanchâtre, couvrant presque tout le fruit et porte des écailles dont les inférieures sont espacées, ovales, apprimées, les moyennes espacées, à partie libre allongée, étalée, un peu en hameçon, les supérieures plus rapprochées, étroites, à extrénité grêle, dressée, crépue. 


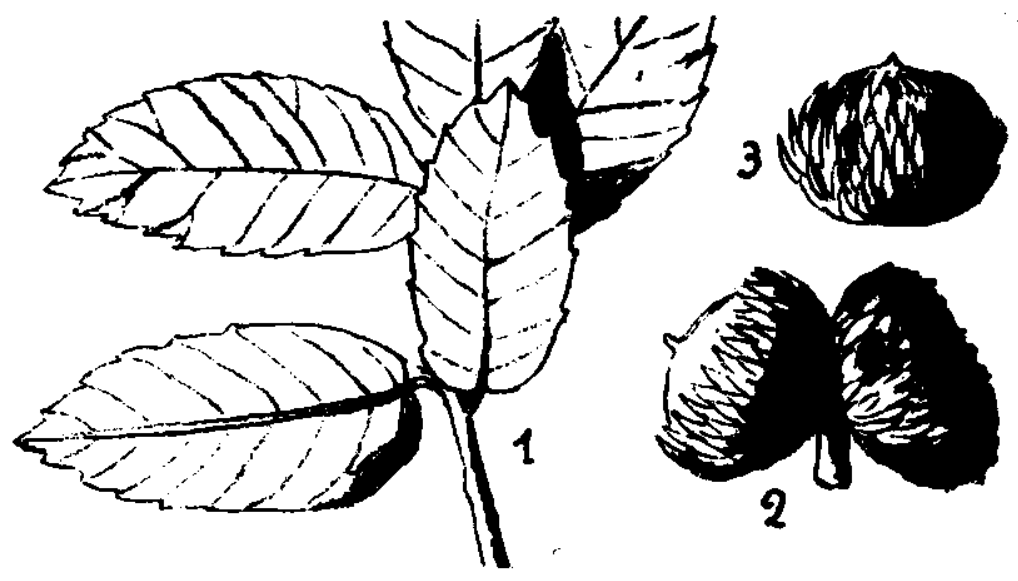

Fig. 13 - Q. calliprinos Webb var. inops DC. - 1 : rameau feuillé. 2 : cupu. les et fruits. 3 : cupule non mûre. (D'après A. Camus).

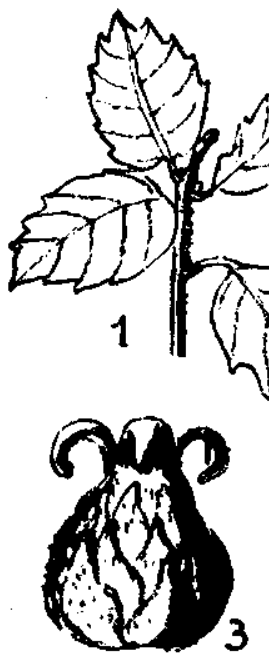

Fig. $14-Q$. calliprinos Webb var. Fenzlii (Kotschy) A. Camus. 1 : rameau feuillé. 2 : cupule et fruit. 3 : jeune fleur femelle très grossie. 4: fleur mâle très grossie.

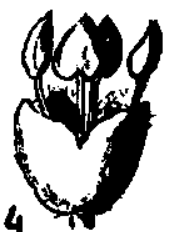

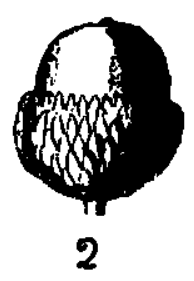

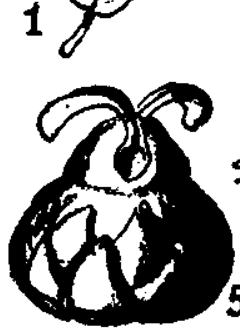

Fig. $15-Q$. calliprinos Webb var. dispar Ky. - 1 : feuille, face supérieure. 2 : cupule et fruit. 3 : fruit. 4 : le même vu de dessous. 5 : fleur femelle très jeune et très grossie. 


\section{2 - Ecailles de la cupule dresses}

var. inops DC. 1864 (Q. inops $\mathrm{Ky}$., 1853)

Feuilles munies de dents petites ; cupule de 10-14 mm de diametre, courte, portant des écailles courtes, soyeuses, blanchatres, brunatres au sommet, larges, souvent obtuses, les médianes longues de 3 à $4 \mathrm{~mm}$, à maturité toutes apprimées, les supérieures infléchies ; fruit petit, souvent imparfait, rarement assez gros, dépassant peu la cupule.

Cette variété est assez répandue dans notre région.

var. Fenzlii A. Camus 1933 (Q. Fenzlii Ky., 1864)

Feuilles ovales ou obovales, à dents assez marquées, dressées ou étaléesdressées; cupule subhémisphérique, un peu resserrée à la gorge, munie d'écailles étroitement imbriquées, dressées, apprimées, ovales, aigues les supérieures plus courtes, lancéolées; gland plus ou moins court, sphérique, parfois ombiliqué au sommet, glabre, cicatrice très convexe.

Cette variété existe dans notre région.

var. subaquifolia A. Camus

Rameaux très tomenteux, à tomentum persistant longtemps. Feuilles grandes, à dents dressées, ressemblant à celles du Houx ; cupule subhémisphérique de $18.20 \mathrm{~mm}$ de diamètre, couvrant le $1 / 3 \mathrm{a} 1 / 4$ du gland, portant des écailles allongées, obtusiuscules, dressées, apprimées, gland ellipsoide très allongé, arrondi au sommet, de $1.1,2 \mathrm{~mm}$ de diamètre, long de $3,5 \mathrm{~cm}$, cicatrice peu convexe.

\subsubsection{Autoécologie et tempérament}

Le Quercus calliprinos est une espèce sociale et héliophile. Ses jeunes semis peuvent supporter le plein découvert. Il ne craint pas le froid; on le rencontre depuis le niveau de la mer jusqu'aux plus hautes altitudes de la région. Il n'est pas exigeant au point de vue édaphique; il arrive à pousser sur des sols extrêmement superficiels et même rocheux en insinuant ses racines dans les fissures, comme on le verra plus loin.

Mais la germination des glands nécessite un sol assez meuble, assez pourvu en eau en surface, mais, une fois installé, le plant envoie ses racines en profondeur quelque soit la nature du sous-sol. Le problème de la régénération naturelle du Quercus calliprinos se pose beaucoup moins que celle du Pinus brutia, parce que le $Q$. calliprinos est une essence qui rejette de souche et peut être regénérée par voie végétative. Le traitement normal des garrigues à chêne kermès dans le Djebel Alaouite est le taillis simple. 


\subsection{Pistacia palaestina Boiss. (nom local arabe : Al Boutm)}

Le Pistacia palaestina est le vicariant du Pistacia Terebinthus de la Méditerranée occidentale. Certains botanistes l'ont réduit quelquefois à une simple variété ou sous-espèce de ce dernier (Pistacia Terebinthus L. ssp. palaestina Eng.): Post 1932 (Flora of Syria, Palestine and Sinai); ThIEBaUT 1940 (Fl. Lib. Syr. $1^{e}$ partie, p. 171, 1940). Nos observations en Syrie confirment la position de BoIsster qui a donné une valeur spécifique au pistachier d'Orient: Pistacia palaestina Boiss. (Diagn. Fl. Or. Nov. Ser. 1, $9: 1,1849-$ Fl. Or. $2: 6-1872$ ). C'est également la position de Bouloumx (Fl. Lib.- Syr. 1930, p. 72) et de Paвot (Rapp. min., agr. syr. 1956).

ZoHaRY (1952) (A monographical Study of the Genus Pistacia. Pal. Jour. Bot. vol. V, 187-228) distingue dans la section EuTerebinthus du genre Pistacia deux groupes:

1) groupe Kintjuk : qui se distingue par des feuilles imparipennées avec une foliole terminale plus large ou égale aux folioles latérales et comprend : Pistacia Kintjuk, Pistacia vera et Pistacia Terebinthus.

2) groupe chinensis : qui se distingue par des feuilles paripennées ou se terminant par un mucron et comprend Pistacia palaestina, Pistacia chinensis (Chine, Formosa, Philippines).

Les caractères suivants permettent de distinguer Pistacia Terebinthus L. et Pistacia palaestina Boiss.

Pistacia palaestina Boiss.

I. feuilles généralement paripennées ou terminées par une pointe ou un mucron

2. folioles généralement acuminées ou mucronéess

3. rachis généralement pubescent
Pistacia Terebinthus L.

I. feuilles généralement imparipennées ; foliole terminale plus large ou égale aux folioles latérales

2. folioles non acuminées

3. rachis généralement glabre.

\subsubsection{Caractères botaniques et dendrologiques}

Le Pistacia palaestina est un arbuste ou un petit arbre de 5 à 10 mètres de haut. Les feuilles sont caduques, glabres, généralement pàripennées ou terminées par une pointe ou un mucron, 
à 4-6 paires de folioles. Celles-ci sont généralement acuminées ou mucronées; le rachis est généralement pubescent et anguleux.

Les fleurs sont dioĩques, unisexuées, sans pétales, en panicules latérales; celles-ci sont lâches, flexueuses, rameuses, plus ou moins étalées; fleurs mâles à 5 sépales et 5 étamines; fleurs femelles à $3-4$ sépales.

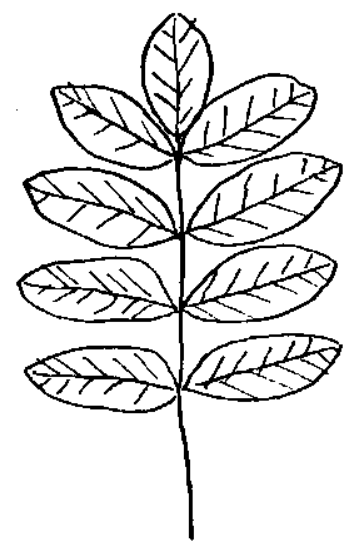

Pistacia Terebinthus L.

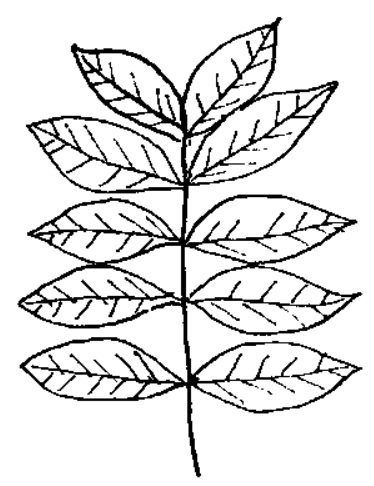

Pistacia palaestina Boiss.

Fig. 16 - Feuilles de Pistacia Terebinthus L. et P. palaestina Boiss.

Le fruit est une drupe presque sèche, à un seul noyau monosperme de 3 à $4 \mathrm{~mm}$, obovale subcomprimé, obliquement mucroné, rouge, puis brun à maturité et comestible.

Le bois à aubier blanc jaunâtre abondant, à coeur brun marron, agréablement nuancé, assez lourd, dur, homogène est susceptible de prendre un beau poli. C'est un bon combustible et il est assez recherché par les ébénistes, sculpteurs et tourneurs.

L'écorce renferme une huile terebenthine. L'amande de la graine contient de l'huile.

\subsubsection{Autoécologie et tempérament}

Le Pistacia palaestina est une espèce héliophile et assez souple du point de vue écologique. En effet, ce Pistacia est indifférent 
à la composition chimique des sols; dans le Nord-Ouest de la Syrie, on le rencontre sur des roches-mères très variées : serpentine, péridotites pyroxéniques, gabbros, dolérites, diorites, marnes crayeuses, calcaire dolomitique, calcaire compact, etc.

Il est résistant au froid; il va du littoral jusqu'à $1500 \mathrm{~m}$ d'altitude dans la Montagne des Alaouites.

Le Pistacia palaestina est très commun dans toute la zone méditerranéenne du Proche-Orient (sauf l'Egypte). En Syrie, il pénètre à l'intérieur du pays jusqu'à la région d'Alep, dans le Kurd Dagh, Jebel Simaan, ainsi que dans l'Anti-liban (Serraya, ouadi El Karn, Hureiré $(1700 \mathrm{~m})$, vallée de Barada jusqu'd Raboué) (PaBor 1956). Il est également répandu dans la région de Banias (Jourdain). Signalons que cette espèce est également répandue au Liban, en Palestine et dans la partie occidentale de la Jordanie où elle fait partie de la garrigue. Sa distribution géographique en Syrie coincide avec celle du Quercus infectoria (Ballout) et du Quercus calliprinos.

En Syrie, on le trouve dans les étages bioclimatiques assez variés: ètages bioclimatiques méditerranéen montagnard inférieur, humide froid, humide tempéré, subhumide et semi-aride.

4. Position du Pistacieto-Quercetum calliprini ( $\left.{ }^{1}\right)$ dans la végetation zonale de la Méditerranée orientale

L'examen des étages de végétation dans la montagne des Alaouites, le Liban et le Mont Galilée nous permettra de préciser la position du Pistacieto-Quercetnm calliprini orientale dans la végétation spontanée zonale.

\subsection{Etages de végettation dans la montagne des alaouites}

\subsubsection{Versant Ouest}

La zone inférieure de la Montagne des Alaouites qui va du niveau de la mer à 200-300 m d'altitude est caractérisée par le

(1) L'association à Querous calliprinos et Pistacia palaestina orientale a été définie par Eig (1937), puis par ZoHARY (1960). 
Caroubier (Ceratonia siliqua) et le Lentisque (Pistacia lentiscus), denx espèces à feuilles persistantes qui constituent le CeratonietoLentiscetum, lequel ressemble à l'Oleo-Lentiscetum de la Méditerranée occidentale. Le Ceratonieto-Lentiscetum est strictement lié au littoral et ne se rencontre pas en Syrie en dehors de cette zone.

La zone située entre 200-300 et 800 mètres d'altitude est occupée par l'association Quercus calliprinos-Pistacia palaestina. Cette

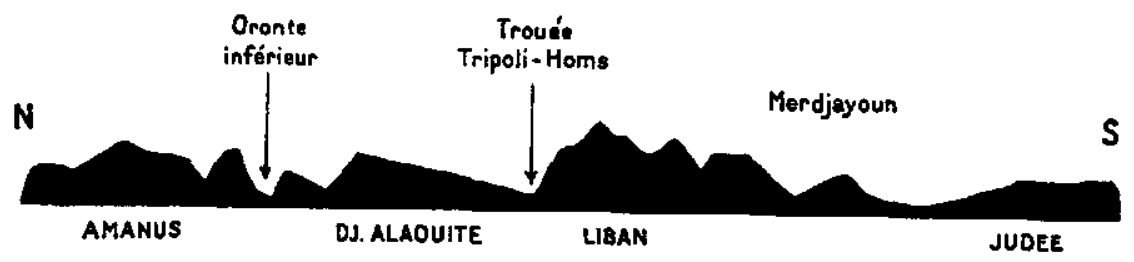

Fig. 17 - Profil longitudinal de la chaîne des massifs de la bordure orientale de la Méditerranée.

association s'enrichit en Quercus infectoria au fur et à mesure qu'on s'élève en altitude et se trouve quelques fois remplacée par le Quercetum infectoriae dans la partie supérieure de cette zone.

Plus haut, entre 850 et $1200 \mathrm{~m}$, apparaît le Quercetum cerridis qui constitue des forêts denses entre 1000 et $1100 \mathrm{~m}$ (Slenfé). Signalons que le Quercus calliprinos s'élève jusqu'à $1500 \mathrm{~m}$ d'altitude mais à l'état d'arbre isolé.

Enfin, la zone supérieure, située entre 1200 et $1500 \mathrm{~m}$ est occupée par l'association du sapin de Cilicie (Abietum cilicicae) (NAHAL 1960).

\subsubsection{Versant Est}

Le versant Est de la Montagne des Alaouites est plus sec que le versant Ouest. Il est occupé dans sa zone supérieure par le Cedretum libani, le Quercetum cerridis et le Quercetum infectoriae et, dans ses zones moyennes et inférieures, par le PistacietoQuercetum calliprini. 


\subsection{Etagho de végétation dans le Liban}

\subsubsection{Versant Ouest}

Le Certatonieto-Lentiscetum occupe la zone inférieure. Le Pistacieto-Quercetum calliprini vient au-dessus et monte jusqu'à $1000 \mathrm{~m}$. Le Quercus infectoria apparait à partir de $800 \mathrm{~m}$ comme arbre disséminé dans le Pistacieto-Quercetum calliprini et le $\mathrm{Pi}$ netum brutiae et monte jusqu'd $1500 \mathrm{~m}$.

Plus haut, entre 1300 et $1500 \mathrm{~m}$ apparaît le Quercus Cerris sous forme d'arbres isolés ou formant des peuplements plus ou moins denses. Il est intéressant de signaler ici que le Pinus brutia envahit d'autres associations dans la zone moyenne et constitue des peuplements assez vastes.

Le Cedretum libani et l'Abietum cilicicae occupent la zone comprise entre 1500 et $1800 \mathrm{~m}$. Le Juniperetum excelsae va jusqu'à $2000 \mathrm{~m}$.

\subsubsection{Versant Est}

Nous retrouvons sur ce versant le Pistacieto-Quercetum calliprini dans la zone moyenne et dans les zones les plus basses nous rencontrons des Amygdalus, Pyrus, Crataegus indiquant un climat nettement plus sec.

\subsection{Etages de végetation dans la Montagne de Galmée}

Dans le Nord de la Palestine, le Pistacieto-Quercetum calliprini se trouve sur le versant Ouest du Mont Galilée et occupe la zone comprise entre 300 et $1200 \mathrm{~m}$, la zone inférieure étant le domaine du Ceratonieto-Lentiscetum.

L'examen des étages de végétation dans la Montagne des Alaouites, le Liban et la Montagne de Galilée nous a permis de préciser la place qu' occupe le Pistacieto-Quercetum calliprini dans la végétation zonale de la Méditerranée orientale : e'est la zone comprise entre la zone de l'association thermophile des feuillus a feuilles persistantes : Ceratonia siliqua et Pistacia Lentiscus (Ce- 
ratonieto-Lentiscetum) et la zone des forêts feuillues à feuilles caduques (Quercetum infectoriae, Quercetum cerridis).

\section{Position du Pistacieto-Quercetum ealliprini du Djebel Alaouite dans le Pistacieto-Quercetum calliprini orientale}

Nous avons vu que le Pistacieto-Quercetum calliprini a une répartition très étendue dans le Proche-Orient, allant de l'Amanus (Turquie) jusqu'au sud de la Palestine et passant par le Cassius, la Montagne des Alaouites (Syrie) et le Liban. Il trouve sa limite inférieure dans le Sud de la Palestine et de la Jordanie, limite à partir de laquelle les espèces méditerranéennes font défant jusqu'aux environs de Derna en Cyrénaique (ZoharY 1960). Ceci est très probablement dû à la très faible quantité de pluies annuelles qui caractérise cette partie de la Région méditerranéenne.

Le Pistacieto-Quercetum calliprini orientale occupe done des étages bioclimatiques et des régions géographiques assez variées. En effet, la pluviométrie moyenne annuelle tombe de $1200 \mathrm{~mm}$ et plus dans l'Amanus, les Alaouites et le Liban à $350 \mathrm{~mm}$ dans le Sud de la Palestine et de la Jordanie.

\section{La composition floristique du Pistacieto-Quercetum ealliprini alaonitaea - Les releves}

\subsection{LoCALISATION DES RELEvÉs}

Les relevés ont été pris sur les deux versants du Djebel Alaonite entre 200 et $800 \mathrm{~m}$ d'altitude. Sur le versant Ouest, les relevés ont été localisés dans une région délimitée vers le Nord par une ligne allant de Lattaquié à Slenfé et vers le Sud, par une ligne allant de Banias, vers le Nord. Sur le versant Est, ils ont été localisés à la base et dans les parties moyenne du Nabi Matta et Nabi Younes. 


\subsection{MÉthode DES RELeVtes}

La méthode que nous avons suivie pour les relevés est celle de Braun-Blanquet. Nous avons noté l'abondance-dominance et la sociabilité, ainsi que la vitalité, la phénologie, le type biologique et les conditions écologiques de la station. Nous avons combiné ensemble abondance et dominance et les avons notées par l'échelle de 1 à 5 comme il suit :

$5=$ Nombre d'individus quelconque recouvrant plus de $3 / 4$ de la surface du relevé

$4=$ Nombre d'individus quelconque recouvrant de $1 / 2$ à $3 / 4$ de la surface

$3=$ Nombre d'individus quelconque recouvrant $1 / 4$ a $1 / 2$ de la surface

$2=$ Individus très abondants ou recouvrant $1 / 20$ a $1 / 4$ de la surface

$1=$ Individus assez abondants, mais degré de reconvrement faible

$+=$ Individus rares ou très rares; recouvrement très faible.

La sociabilité (mode de groupement entre eux des individus de chaque espèce) a été notée également par l'échelle allant de 1 à 5 :

$1=$ Individus isolés

$2=$ Individus groupés

$3=$ Individus en troupes

$4=$ Individus en petites colonies

$5=$ Individus en peuplements.

Les surfaces des relevés ont été déterminées par la méthode classique de l'aire minimale définie par la courbe aire-espèce (e'est l'aire sur laquelle la quasi-totalité des espèces de la communauté végétale est représentée). Cette méthode consiste à faire la liste des espèces sur une surface très faible, puis doubler cette surface et ajouter les espèces nouvelles qui apparaissent. Par doublements successifs on arrive à une surface à partir de laquelle il n'y a pratiquement plus d'espèces nouvelles qui apparaissent. L'aire minimale du Pistacieto-Quercetum calliprini est de $100 \mathrm{~m}^{2}$.

\subsection{CholX Des ReLevís}

Pour le choix des relevés nous nous sommes attachés aux principes suivants :

— homogénéité : la surface du relevé doit être homogène 


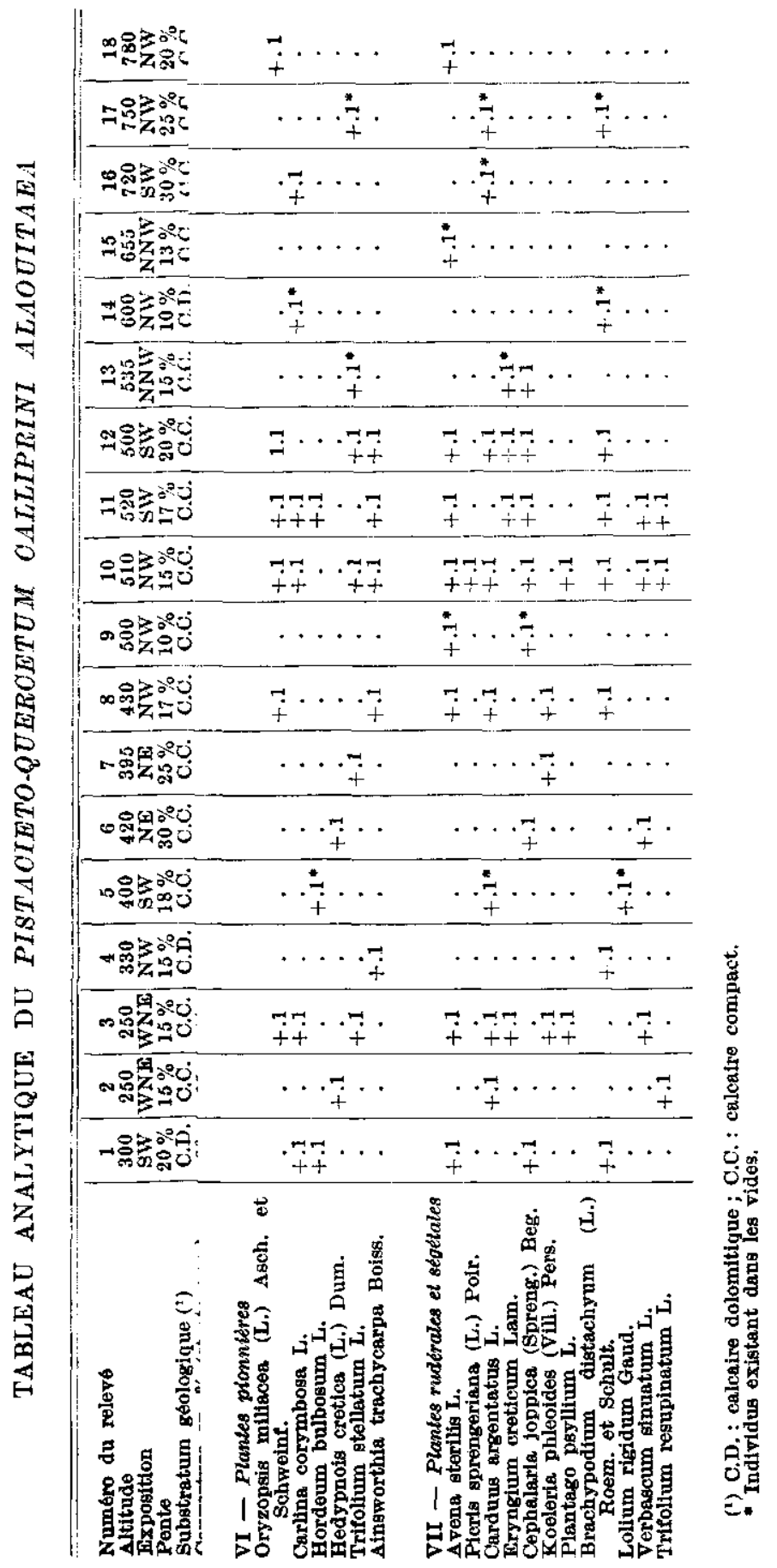


vis-à-vis des critères descriptifs utilisés : structure, composition floristique ou écologique;

- représentativité : les relevés doivent être représentatifs, c'est-à-dire permettre la description complète de la végétation du point de vue envisagé ;

- comparabilité : les surfaces doivent être égales à l'aire minimale de l'association pour faciliter la comparaison.

\subsection{Echantillonage sur le terkain}

Il est difficile d'être objectif dans le choix des échantillons tout en respectant les conditions précédentes. L'échantillonnage phytosociologique que nous avons effectué est subjectif, c'est-à-dire que nous avons choisi, en fonction de notre connaissance du milieu, les surfaces à relever répondant aux conditions d'homogénéité, de représentativité et de comparabilité. Cette méthode, bien que subjective, est justifiable car ce qui est important c'est l'étude des surfaces homogènes et représentatives. Si on choisissait au hasard des surfaces de l'ordre de l'aire minimale, on a de fortes chances de tomber sur des surfaces floristiquement et écologiquement hétérogènes. Dans ces conditions, les comparaisons n'ont plus de sens du point de vue de la définition des unités de végétation.

$\mathrm{Il}$ est important de signaler ici que si même le choix des échantillons est dirigé, il est bien difficile de le diriger complètement et de tenir compte dans le choix de ces échantillons de tous les facteurs considérés.

\section{Position syst6matique du Pistacieto-Quercetum calliprini}

La classe des Quercetea calliprini est subdivisée en trois ordres que nous résumons dans ce qui suit :

\section{Classe : Quercetea calliprini}

I. Ordre Quercetalia calliprini

II. "Poterietalia spinosi

III. " Ballotetalia undulatae 
L'ordre des Quercetalia calliprini renferme plusieurs alliances :

- Quercion calliprini

- Pinion brutiae (Syrie, Turquie, Chypre)

- Pinion halepensis

- Ceratonio-Pistacion Lentisci

Sous l'alliance Quercion calliprini (ZoHARY 1947), on groupait les 3 associations suivantes:

1. Association à Quercus calliprinos et Pistacia palaestina (EIG 1927, 1938) (garrigue) ; répandue en Syrie, Liban, Turquie, Chypre, Palestine.

2. Association à Ceratonia siliqua et Pistacia Lentiseus (Erg 1939) (garrigue); répandue sur le littoral de Syrie, Liban, Palestine, Turquie.

3. Association à Quercus calliprinos et Juniperus phoeniced (FEINBRUN et Zohar 1942); répandue en Palestine et dans le Sud de la Jordanie.

Nous groupons sous la même alliance le "Quercetum calliprini ", qui représente la forêt climacique dans la montagne des Alaouites entre 200-300 et $800 \mathrm{~m}$ d'altitude et qui est l'aboutissement de l'évolution de la garrigue à Quercus calliprinos et Pistacia palaestina, comme on le verra plus loin, non seulement dans notre région, mais partout dans le Proche-Orient.

\section{Subdivisions du Pistacieto-Quercetum calliprini en Syrie}

En Syrie, on rencontre le Pistacieto-Quercetum calliprini dans les régions suivantes : 1 ) dans le Nord-Ouest et plus particulièrement dans la Montagne des Alaouites, où il occupe les étages méditerranéens bioclimatiques subhumides et humides. Du point de vue floristique cette région se distingue par sa grande richesse floristique. 2) dans le Nord, et particulièrement dans le Kurd Dagh où il se situe dans l'étage bioclimatique semi-aride. Floristiquement cette région est moins riche en espèces que la précédente. 3) dans le Sud (sources du Jourdain) où il se situe dans l'étage bioclimatique semi-aride. Du point de vue floristique, cette région se distingue par l'absence de certaines espèces fréquentes dans le Nord-Ouest comme : Arbutus Andrachne, Phillyrea media, Erica verticillata, Fontanesia phillyreoides, Gonocytisus pterocladus, Spartium junceum, Cercis siliquastrum, etc. 
L'association du Quercus calliprinos et Pistacia palaestina de Syrie peut être subdivisée, tant du point de vue floristique que bioclimatique en trois sous-associations :

1) Sous-association de la région côtière ou de la Montagne des Alaouites : "Pistacieto-Quercetum calliprini Alaouitaea*. Dans cette sous-association nous pouvons distinguer deux variantes suivant l'altitude :

A) Une variante, dans la partie supérieure de I'étage du Quercetum calliprini, qui renferme des représentants de l'étage des feuillus a feuilles caduques : Quercus infectoria, Prumus ursina, Laurus nobilis, Acer syriacum, etc.

B) Une variante, dans la partie inférieure de l'étage du Quercetum calliprini, qui est plus paurre en espèces à feuilles caduques et plus riches en especes thermophiles.

2) Sous-association du Nord de la Syrie ou du Kurd-dagh : "PistacietoQuercetum calliprini Syro-septentrionalis ".

3) Sous-association du Sud de la Syrie: "Pistacieto-Quercetum calliprini Syro-meridionalis \#.

\section{Caractéristiques synécologiques du Pistacieto-Quercetum calli- prini Alaonitaea.}

\subsection{EtTages biochimatrques}

La garrigue à Quercus calliprinos et Pistacia palaestina du Djebel Alaouite se situe dans les étages bioclimatiques méditerranéen, subhumide et humide, ce qui correspondrait à une pluviosité annuelle allant de 700 à $1000 \mathrm{~mm}$.

\subsection{SoLs}

La garrigue à Quercus calliprinos et Pistacia palaestina se rencontre essentiellement sur Terra rossa : terre argileuse rouge remplissant les fissures et les excavations du calcaire compact. Ces fissures sont généralement assez larges du fait de l'action corrosive des eaux de pluie. L'ensemble constitue un sol discontinu, le calcaire compact affleurant en surface sur des étendues plus ou moins grandes. Les propriétés des sols formés sur Terra rossa dépendent de la densité et de l'ancienneté de la garrigue : sous les peuplements denses on aperçoit un léger horizon $A_{1}$ brun, 
légèrement humifère (0,5-1\% d'humus) contrastant avec l'horizon $\mathbf{A}_{2}$ rouge. Sous les peuplements très ouverts, l'horizon $\mathbf{A}_{1}$ fait défaut et le sol montre une rubéfaction dans les horizons de surface; le sol est très sec et se trouve colonisé par les espèces xérophiles épineuses (Calycotome villosa, Genista acanthoclada, Poterium spinosum).

Le Pistacieto-Quercetum calliprini se trouve également, mais moins fréquemment, sur marne crayeuse, calcaire marneux et calcaire dolomitique.

10. La signification 6eologique de la composition floristique de la garrigue à Quercus calliprinos et Pistacia palaestina

Les relevés que nous avons effectués dans des localités assez variées nous ont permis de savoir que le Pistacieto-Quercetum calliprini de la Montagne des Alaouites est une association exceptionnellement riche en espèces et que sa composition floristique était, en général, un mélange d'espèces caractéristiques propres au Quercion calliprini (Phillyrea media, Rhamnus palaestina, $P i$ stacia Lentiscus, Styrax officinale, Ceratonia siliqua, Amygdalus communis, Olea europaea, Smilax aspera, etc.) et au PistacietoQuercetum calliprini (Laurus nobilis, Arbutus Andrachne, Quereus infectoria, Spartium junceum, Rhamnus Alaternus, Cercis siliquastrum, ete.) et d'espèces caractéristiques des associations qui le succèdent pendant les phases de dégradation ou qui le précèdent au cours de l'évolution progressive vers le climax. Ce mélange est évidemment dû à l'intervention continuelle de l'homme. La présence d'espèces autres que les caractéristiques de l'alliance et de l'association a una grande signification écologique et dont la connaissance est bien utile aux forestiers, -car ces espèces nous permettent de tracer l'histoire de l'association, déterminer si nous sommes dans une phase d'évolution vers le climaux ou dans une phase de dégradation et enfin elles nous renseignent sur les conditions stationnelles et plus particulièrement sur les conditions édaphiques. En voici des exemples :

1 - La haute présence d'espèces appartenant au Poterietum spinosi (comme : Poterium spinosum, Helichrysum sanguineum, Origanum syriacum) indique un état très dégradé du sol et des conditions de sécheresse accrue du sol. 
2 - L'extension d'espèces caractéristiques du Cistetum (comme : Cistus villosus, Oistus salviaefolius, Thymbra spicata, etc.) indique un début de dégradation du Pistacieto-Quercetum calliprini et reflète des conditions de sécheresse assez élevée du sol ; renseignement très utile pour le forestier de notre région.

3 - L'absence d'espèces appartenant au Poterietum et an Cistetum qui sont très exigentes en lumière (ou leur faible présence) indique un sol plus évolué, mieux pourva en humus et en eau.

4 - L'extension d'espèces rudérales et ségétales (Plantago psyllium, Cephalaria joppica, Brachypodium distachyum, Avena sterilis, Cardurs argenteus, Eryngium creticum, Verbascum simuatum, etc.) indique des sols récemment abandonnés par la culture à l'intérieur de la garrigue. Ceci présente un intérêt pour les forestiers syriens qui ont à déceler les déboissements illégaux pratiqués par les paysans pour la mise en culture des sols.

5 - L'extension de certaines espèces ligneuses épineuses comme Oalycotome villosa et Genista acanthoclada, qui sont en principe de faible valeur fourragère et dédaignées par le bétail, indique que nous sommes dans une phase d'évolution regressive. En effet, il suffit d'interdire le pâturage dans de telles zones pendant quelques années pour voir disparaître graduellement ces espèces épineuses et les voir remplacer par certains arbustes et arbrisseaux appartenant à la série progressive.

6 - La présence d'espèces caractéristiques du Quercetum infectoriae nous indique des conditions d'altitude et de pluviosité assez élevées, ren. seignement très important dans les grandes régions forestières où les stations météorologiques font défaut.

\section{Facteurs de variation de la composition floristique de la garrigue}

La composition floristique de la garrigue dépend des facteurs suivants :

\subsection{Altitude}

Nous avons vu plus haut que le Pistacieto-Quercetum calliprini occupe une zone assez étendue en altitude allant de 200-300 m à $800 \mathrm{~m}$. Dans la partie inférieure de cette zone, il aura des affinités avec le Ceratonieto-Lentiscetum et constituera une variante pauvre en espèces à feuilles caduques, alors que dans la partie supérieure de cette zone il aura des affinités avec l'étage des forêts à feuilles caduques (Quereus infectoria, Quercus Cerris) et constituera une variante plus riche en espèces à feuilles caduques, Quercus infectoria, Laurus nobilis, Acer syriacum, etc. 


\subsection{ExposItion}

Le Pistacieto-Quercetum calliprini existe aussi bien sur le versant Onest que sur le versant Est de la Montagne des Alaouites. Sur ce dernier, il se montre plus xérophile et moins riche en espèces à feuilles caduques, mais en revanche il s'y montre plus sensible à la dégradation que sur le versant Ouest étant donné la sécheresse et les températures plus excessives. L'imprudence de l'homme, avec ses coupes abusives, $y$ aboutissent très vite à des désastres. Dans l'ensemble, cette association est plus dégradée sur le versant Est et se trouve plus riche en espèces appartenant aux Cistetum et Poterietum.

\subsection{ORIGINE DE LA GARRIGUE}

La composition floristique de la garrigue varie suivant son origine, c'est-à-dire suivant qu'elle résulte de la dégradation directe de la forêt primitive de Quercus calliprinos (Quercetum calliprini) ou qu'elle constitue un stade assez avancé de la réoccupation du sol nu par la végétation spontanée dans le sens d'une évolution vers le climax (Quercetum calliprini). Dans le deuxième cas, la garrigue renfermera plus ou moins d'espèces caractéristiques des associations qui l'ont précédée dans le temps (Poterietum spinosi, Cistetum) suivant son ancienneté d'installation.

\subsection{Densité DE LA GARRIGUE}

La garrigue dense sera plus pauvre en espèces et sera constituée presque exclusivement d'espèces caractéristiques de l'alliance (Quercion calliprini) et de l'association. Elle sera entièrement dépourvue d'espèces appartenant au Poterietum et au Cistetum.

\section{Phǿnologie}

Les phanérophytes et les chaméphytes sont les formes les moins répandues dans la garrigue à chêne kermès, bien que ce 
soient les phanérophytes qui lui donnent sa physionomie propre. En effet, leur nombre ne dépasse pas $10-12 \%$ du nombre total des espèces présentes dans la garrigue. Le reste, $88-90 \%$ est partagé entre les thérophytes, hémicryptophytes et cryptophytes.

Les phanérophytes sont soit à feuilles persistantes, soit à feuilles caduques, mais, chose curieuse, le nombre des phanérophytes à feuilles caduques est égal ou légèrement supérieur à celui des phanérophytes à feuilles persistantes. Ceci paraît anormal dans une région où l'hiver est doux et pluvieux, par conséquent, favorable à l'activité physiologique des végétaux. Mais ces espèces à feuilles caduques se montrent souples vis-à-vis des variations climatiques et adaptent leur période de repos à la rigueur plus ou moins grande de la saison hivernale.

\section{Adaptation des espèces de la garrigue au substratum rocheux caleaire}

Nous avons vu que la garrigue à Quercus calliprinos et Pistacia palaestina se trouvait surtout sur Terra rossa, terre argileuse rouge remplissant les fissures et les excavations du calcaire compact. Les racines des espèces arbustives qui la composent s'insinuent dans ces fissures et vont quelquefois jusqu'à 3 à $6 \mathrm{~m}$ de profondeur à la recherche de l'eau qui s'accumule en profondeur à l'abri de l'évaporation. Ces espèces sont adaptées à ces conditions édaphiques et envoient leurs racines dans deux directions principales: direction horizontale pour l'utilisation de l'eau superficielle pendant la période humide et direction suivant les fissures pour l'utilisation de l'eau accumulée en profondeur pendant la saison chaude et sèche au moment où l'eau superficielle disparaît par évaporation. C'est ainsi qu'on a observé des racines allant jusqu'à $5 \mathrm{~m}$ de profondeur chez Quercus calliprinos, Phillyrea media, Ceratonia siliqua, Arbutus Andrachne, etc. dans les carrières d'extraction $d u$ calcaire compact. On a même observé dans certains cas, des racines des espèces précédantes ayant pénétré dans la roche dure intacte.

Certains ont même observé des graines de Pistacia Lentiscus, Rhamnus palaestina, Phillyrea media ayant germé dans des cavités 
minuscules sur roche calcaire intacte. Ces phénomènes sont assez rares dans la nature, mais sont suffisantes pour nous montrer la rusticité des principales espèces arborescentes qui composent la garrigue et leur résistance à l'érosion et nous permettent d'expliquer comment ces espèces ont pu subsister malgré l'action destructrice et continue de l'homme depuis des millénaires.

En principe, la germination des semences de ces espèces ne se fait que sur sol meuble et assez bien pourvu en réserves hydriques. Mais, après la germination, et une fois que la plantule est installée, elle envoie ces racines en profondeur à la recherche de l'eau. En général, les espèces qui composent la garrigue se montrent bien adaptées au substratum calcaire rocheux et sec, d la sécheresse atmosphérique et aux hautes températures sui sévissent en été.

\section{Successions phytodynamiques}

La garrigue à Quercus calliprinos et Pistacia palaestina se dégrade sous l'influence des coupes abusives de taillis et du pâturage excessif et cède la place à d'autres associations plus xérophiles et protégeant moins le sol. Un sol abandonné par la culture dans la région étudiée, se trouve envahi par des associations qui se succèdent jusqu'au stade climax.

Pour trouver ces phases de successions progressives et regressives nous avons eu recours à deux méthodes: 1) l'observation de l'installation de la végétation spontanée sur les terres abandonnées par la culture à des dates échelonnées du fait de leur épuisement ; 2) la recherche dans l'espace des phases de succession dans le temps.

Il convient de signaler que la nature des associations qui se succèdent dépend de la nature de la roche-mère (Terra rossa, calcaire dolomitique, marne). Mais comme la garrigue à Quercus calliprinos est essentiellement sur Terra rossa, nous avons étudié les phases de succession sur cette dernière roche-mère. 


\subsection{EvOLUTION PROGRESSIVE}

\section{1ère phase: Les Pionniers}

Après abandon des cultures, le sol se trouve envahi par des espèces pionnières, très souples du point de vue écologique et pouvant supporter des conditions de vie sévères.

En effet, ces sols abandonnés par la culture sont très érodés, très secs et dépourvus totalement de matière organique et souvent la roche calcaire affleure à la surface. Au début, les espèces pionnières se trouvent mélangées avec des espèces qui accompagnaient les plantes cultivées mais qui ne tardent pas à disparaitre quelques années plus tard.

Parmi les pionniers nous pouvons citer : Hordeum bulbosum, Dactylis glomerata, Carlina corymbosa, Phalaris tuberosa, Fumana arabica, Oryzopsis sp., etc.

2ème phase: Pelouse sèche à Hyparrhenia hirta (Hyparrhenietum hirtae)

Cette phase se caractérise par l'abondance d'une graminée, Hyparrhenia hirta, pouvant supporter des conditions de sécheresse accrue du sol. Elle est accompagnée de Pollinia distachya, Thymbra spicata, Andropogon Ischaemum, etc.

3ème phase: Lande à Poterium spinosum (Poterietum spinosi)

C'est une lande formée essentiellement de Poterium spinosum, sous-arbrisseau épineux s'accrochant fermement au sol. Il est accompagné des espèces suivantes : Origanum syriacum, Helichrysum sanguineum, Dactylis glomerata, etc.

Toutes ces espèces sont xérophiles et jouent un grand rôle dans la fixation du sol et la préparation d'un milieu favorable à d'autres associations plus exigeantes du point de vue édaphique. C'est ainsi, qu'il est de toute importance d'interdire complètement l'arràchage du Poterium spinosum que les villageois utilisent pour le chauffage.

En effet, le Poterietum spinosi est l'une des principales phases de l'occupation du sol et l'évolution vers le climax. Sa destruction 
signifie l'arrêt de l'évolution progressive et la destruction continue du sol.

4ème phase: Lande à Cistus villosus et Cistus salviaefolius (Cistetum)

Le Cistetum nécessite pour s'installer un certain abri que lui offre le Poterium spinosum. Il est essentiellement formé de Cistus villosus, Cistus salviaefolius, accompagnés de Teucrium creticum, Calycotome villosa.

A ce stade de l'occupation du sol par la végétation spontanée, on commence à apercevoir quelques débris de matière organique accumulés sur le sol. Ce dernier est en général plus couvert et mieux pourvu en eau qu'aux stades précédents.

5ème phase : garrigue à Querous calliprinos et Pistacia palaestina (Pistacieto-Quercetum calliprini)

Les espèces de la garrigue remplacent graduellement les espèces du Cistetum, qui sont très héliophiles, jusqu'à les éliminer complètement. Mais ce remplacement dure un temps assez long, car il nécessite un sol assez bien pourvu en humus et en réserves hydriques pour que la germination des semences et, en particulier, les glands de Quercus calliprinos qui sont très sensibles à la sécheresse $d u$ sol, puisse avoir lieu.

Dans cette phase, un horizon $A_{0}$, dont l'épaisseur varie suivant la densité du peuplement et l'ancienneté de l'installation de la garrigue, apparaît à la surface du sol.

Un autre fait nouveau : c'est l'individualisation apparente d'un horizon $A_{1}$ légèrement humifère et de covleur brune, contrastant avec la couleur rouge des horizons inférieurs. Un sol brun méditerranéen jeune s'est done formé sur Terra rossa et sous la garrigue. Signalons que ce sol brun ne se manifeste que dans les poches remplies de terre fine, partout ailleurs la roche calcaire affleure à la surface.

6ème phase : forêt de Querous calliprinos (Quercetum calliprini) - olimax

La forêt de Quercus calliprinos représente la dernière phase de cette évolution progressive de la végétation spontanée sur 
I. Nahas, Contribution à l'étude de la régitation du bacr-Rassit et du Iti. Alaouite (Syrie).

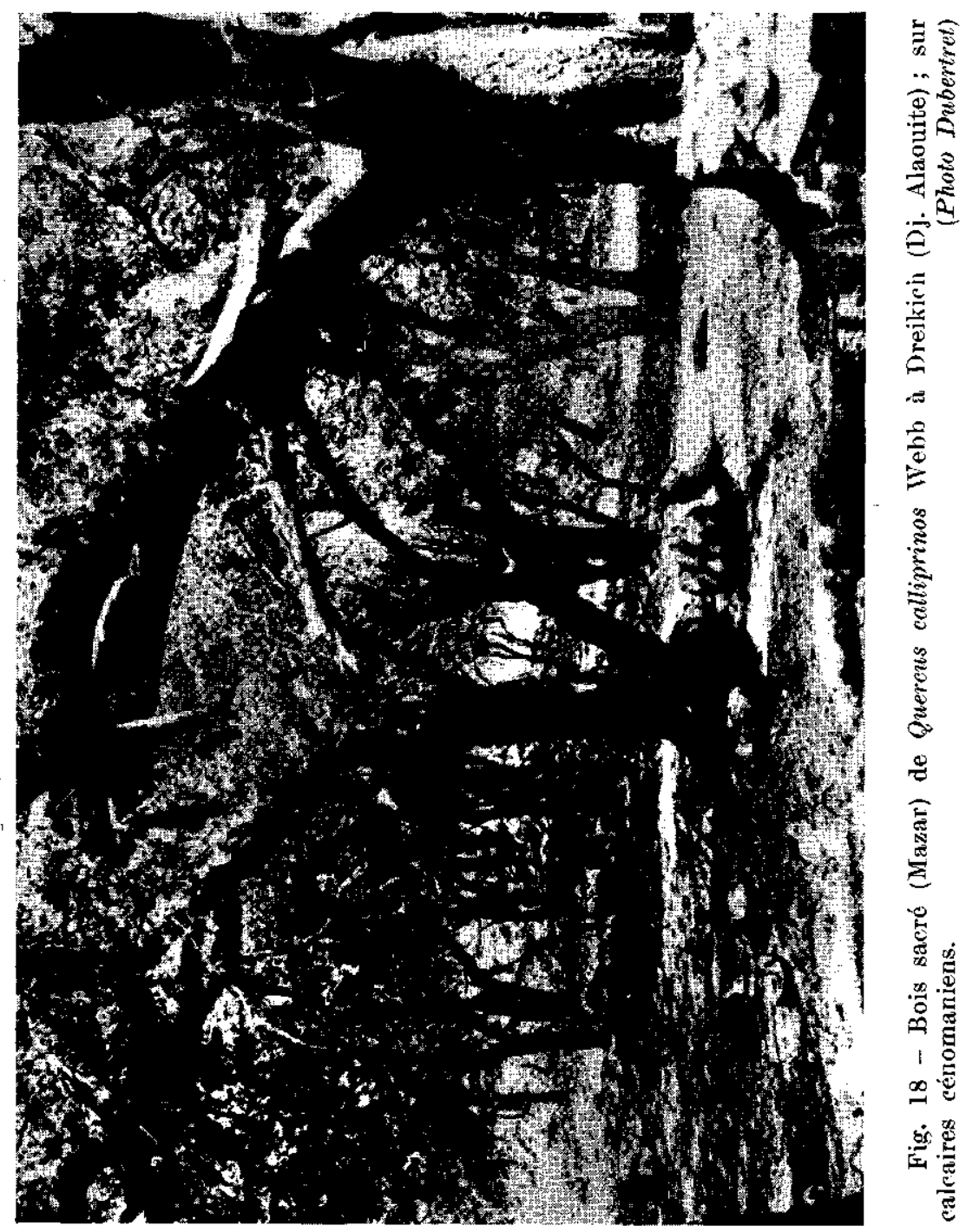


Terra rossa et dans la zone moyenne de la Montagne des Alaouites. Nous avons étudié ce stade final dans les bois Sacrés de Querous calliprinos situés autour des "Mazars" où on trouve des peuplements d'âges variés suivant l'ancienneté du Mazar (Cheikh Has-
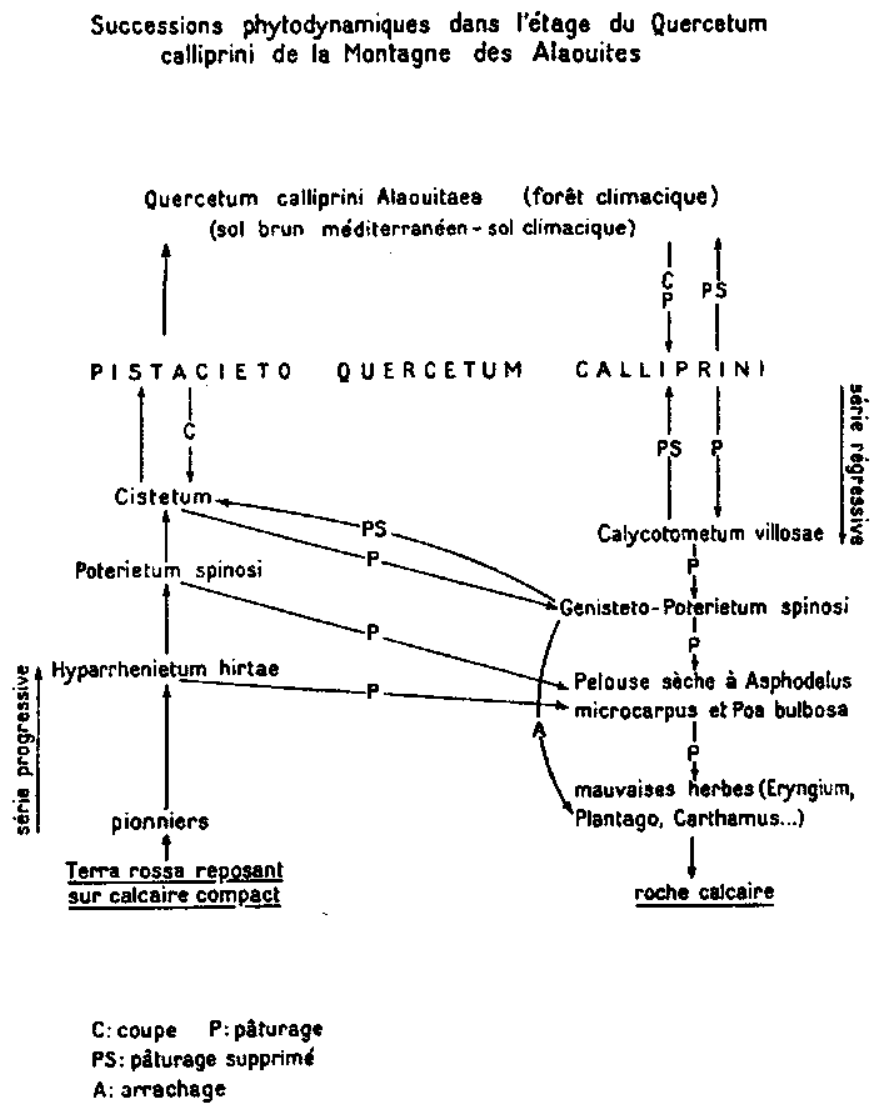

samo, par ex.). La présence de ces bois sacrés d'âges variés permet de suivre l'évolution de la garrigue vers le climax.

En effet, une fois protégée, la garrigue évolue et sa composition floristique passe d'un mélange très riches en espèces à un peuplement presque pur et très dense de Quercus calliprinos, composé d'arbres ayant 10-15 m de haut et accompagné de Quercus infectoria, Laurus nobilis, Pistacia palaestina. Sous ce 
peuplement l'horizon $A_{0}$ atteint $2-3 \mathrm{~cm}$ d'épaisseur et l'horizon $\mathbf{A}_{1}$ brun humifère renferme $2-3 \%$ d'humus et contraste avec les horizons rouges inférieurs. Le stade final de l'évolution du sol dans cette zone sur Terra rossa est un sol brun méditerranéen.

\subsection{EVOLUTMON REGRESSIVE}

Sous l'influence des coupes abusives et surtout du pâturage excessif et non réglementé de la chèvre dans ces garrigues, le Pistacieto-Quercetum calliprini se dégrade et se trouve remplacé par des associations plus xérophiles à base d'épineux, souvent dédaignés par les animaux comme: Calycotome villosa, Genista acanthoclada. C'est ainsi qu'une lande à Calycotome villosa remplace la garrigue, et si le pâturage continue, cette lande se trouve remplacée par une lande à Poterium spinosum et Genista acanthoclada beaucoup plus xérophile et plus elaire que la précédente, puis c'est une pelouse sèche à Asphodelus microcarpus et finalement des mauvaises herbes des terres incultes (Eryngium, Plantago, Carthamus, etc.) qui prennent possession du terrain.

Le sol suit la même dégradation : l'horizon $\mathbf{A}_{1}$ disparaît graduellement, puis la terre fine, jusqu'à l'apparition de la roche calcaire.

15. Importance de la connaissance des phases de succession phytodynamiques pour le forestier

La connaissance des "variantes" du Pistacieto-Quercetum calliprini et des associations qui se succèdent dans l'évolution vers le climax et dans la dégradation de la forêt primitive est très utile au forestier. Ces variantes et ces associations reflètent fidèlement les conditions du milieu (altitude, pluviométrie, sol). Les conditions édaphiques sont reflétées plus particulièrement par les associations de dégradation qui nous renseignent sur la profondeur du sol, sa richesse en humus et surtout sur sa teneur en eau, notions très utiles pour les reboiseurs.

En voici quelques exemples concrets :

1) Dans la variante chaude de "basse altitude du PistacietoQuercetum calliprini on peut envisager l'introduction des espèces 
suivantes pour le reboisement : Eucalyptus camaldulensis, sur les sols profonds non calcaires; Eucalyptus gomphocephala, sur les sols profonds même calcaires; Acacia cyanophylla, sur les sols plus superficiels. Nous pouvons même envisager des plantations d'oliviers et de figuiers.

2) Dans la variante froide du Pistacieto-Quercetum calliprini on peut envisager l'utilisation des espèces suivantes pour le reboisement : Eucalyptus viminalis et $\boldsymbol{E}$. gunnii, sur les sols profonds; Pinus nigra pallasiana, Pinus brutia, sur les sols moins profonds. En matière d'arboriculture, nous pouvons envisager des plantations de pommiers (variétés américaines) et des cerisiers après établissement de terrasses et utilisation des explosifs pour provoquer des fissures artificielles dans les roches afin d'activer la pédogénèse et faciliter la pénétration des racines. Les soussolages sont à conseiller également.

3) Dans le Poterietum et le Cistetum, les reboisements nécessiteront une bonne préparation du sol et des soins particuliers aux jeunes plants avant et après le reboisement. Des arrosages sont quelques fois nécessaires, si la saison qui suit la plantation n'est pas suffisamment humide. Les reboisements en pins par voie de semis est à prescrire dans ces landes, car ils seront voués à un échec certain.

4) Les chances de réussite des reboisements dans le Calycotometum sont plus élevées.

5) La plantation de pins dans le Calycotometum ou le Cistetum, favorisera l'installation graduelle des espèces du Pistacieto-Quercetum calliprini qui constitueront ainsi un sous-étage de feuillus sous la forêt artificielle de pins, nécessaire au maintien de la fertilité de la station alors que les pins constitueront l'avenir proche $\mathrm{du}$ point de vue économique. Ce mariage entre resineux et feuillus est à souhaiter partout dans le Proche-Orient.

6) Une mise en défens de quelques années de la lande à Cistus villosus et Cistus salviaefolius suffira pour voir apparaître des éléments $d u$ Pistacieto-Quercetum calliprini.

7) Des coupes appropriées permettront de faire évoluer la garrigue vers une forêt de Quercus calliprinos. 


\section{BIBLIOGRAPHIE}

Liste des abréviations utilisées dans la Bibliographie:

C.N.R.S. = Centre National de la Recherche Scientifique

E.N.E.F. = Ecole Nationale des Eaux et Forêts

R.E.F. = Revue des Eaux et Forêts

R.F.F. = Revue Forestière Française

SIGMA = Station Internationale de Géobotanique Méditerranéenne et Alpine.

AlBRight W. F., 1960. The Archeology of Palestine. London, $271 \mathrm{p}$.

Aubert G., 1954. Les sols latéritiques. C. R. du Vo Congrès intern. Sc. du Sol, Leopoldville, I, pp. 103-118.

AUbert G. et Monjadze A., 1946. Observations sur quelques sols de l'Oranie nord-occidentale. Influence du déboisement et de l'érosion sur leur évolution. C. R. Séances Soc. Biogéogr. 23, 199, pp. 44-51.

Aubert G., Rosead H., Gadcher G. et Y. YNkovitch L., 1947. Lez bols à croute calcaire. C. R. Confér. pédol. médit., Alger-Montpellier, pp. 330-337.

BAUDrike A. et EMBerger L., 1959. Sur la notion de climat de transition en. particulier dans le domaine du climat méditerranéen. Bull. Serv. Carte. Phyt., série B, Tome IV, 2, pp. 95-117.

BRRENGer H., 1955. Essai d'étude météorologique du bassin méditerranéen. Mémorial de la Météo. Nat., 41 p.

BERJAOUI A., 1952. La distribution des essences forestières au Liban. R.F.F. décembre 1952, pp. 833.837.

Birot P. et Dresch J., 1953-1956. La Méditerranée et le Moyen-Orient. 'T. I : la Mediterranée occidentale, 544 p. - T. II : la Méditerranée orientale. et le Moyen-Orient. 526 p. Presses Universitaires de France, Paris.

Borssier E., 1867-88. Flora Orientalis. 5 vol. et suppl. Genevae.

BORDaS J., 1943. Essai de pédologie méditerranéenne. Imprimerie Nationale, Paris.

BoUDY P., 1950. Monographie et traitement du Cedre. In : Economie Forestiere. Nord-Africaine, T. II, 2, pp. 529-619. Ed. Larose, Paris.

- 1950. Association du Pinus halepensis en Afrique du Nord. T. I, p. 132. Monographie et traitement du pin d'Alep. T. II, 1, p. 639. - In : Economio. Forestiere Nord-Africaine.

Boulos L., 1959. A Contribution to the Flora of Gaza Zone. Agricult. Extension Dept., Ministry Agricult., Cairo.

- 1960. Cytotaxonomic Studies in the Genus Sonchus. 2. The Gemus Sonchus, a General Systematic treatment. Botaniska Notiser, 113 (4) : 400-420.

- 1960. Flora of Gebel El-Maghara North Sinai. Agricult. Extension Dept., Ministry Agricult., Cairo.

Bouloumor L., 1930. Flore du Liban et de la Syrie. Vigot Frères, Ed.

Braun-Blanquet J., 1934. Association végétale climatique et climax du sol. dans le midi méditerranéen. R.E.F., T. LXXII, pp. 1-7.

Braun-Blanquet J., Fullet H. S. and Conard G. D., 1932. Plant Sociology (authorized English transalation of Pflanzensoziologie). Ed. 1. 
Campo.Duplan (van), 1950. Recherches sur la phylogénie des Abiétinées d'après leurs grains de pollen. Trav. Lab. Forest. Toulouse, T. II, vol. IV, Art. 1, Toulouse.

Cayrus A., 1936-1954. Monographie du Genre Quereus. Texte et Atlas. T. I; 1936-38 ; T. II : 1938-39 ; T. III : 1952-54 ; Paul Lechevalier et fils, Paris.

Chfнaвi M., 1943. Dictionnaire Français-Arabe des Termes Agricoles. Damas, $656 \mathrm{p}$.

Cure P., 1943. Les essais de réprésentations synthétiques des climats pour la géographie botanique. Documents pour la carte des productions végétales. Série : généralités, vol. l, art. 1.

Czeczort H., 1954. The past and present distribution of Pinus halepensis Mill. and Pinus brutia Ten. C. R. Cong. Bot. Paris, vol. des sect. 2-6, pp. 196-197.

- 1939. A contribution to knowledge of the Flora and Vegetation of Turkey. F. Fedde Rep. spec. nov. regni veg., Bei., Band 107, Berlin-Dahlem.

DANSEREAU P., 1957. Biogeography - an ecological perspective. The Ronald Press Comp., New York.

DaUBenMiRe R. F., 1959. Plants and environment. John Wiley and sons, Inc. New York, $422 \mathrm{p}$.

Deacon E. L., Priesteme C. H. B., Swinbank W. C., 1958. Evaporation and the water balance. In : Climatology, review of research UNESCO, pp. 9-34.

Debazac F., 1959. La végétation forestière dans la Kroumirie. Ann. E.N.E.F., T. XVI, 2.

Demolon A., 1956. Oroissance des végétaux cultivés. Ed. 5, Dunod, Paris, $576 \mathrm{p}$.

- 1960. Dynamique du 8ol. Ed. 5, Dunod, Paris, 520 p.

DRdDE O., 1897. Handbuch der Pflanzengeographie. Stuttgart, J. Engelhorn (transl. G. Poirault as Manuel de Gégraphie Botanique), Paris, Lib. Se. Nat.

DUBertret L., 1946-1948. Aperçu de géographie physique sur le Liban, l'Antiliban et la Damascène. Etudes géogr. et géol. sur le Liban, la Syrie et le Moyen-Orient, T. IV, pp. 191-226.

- 1953. Géologie des roches vertes du Nord-Ouest de la Syrie et du Hatay (Turquie). Extr. Notes et Mém. sur le Moyen-Orient, T. VI, Paris.

- 1953. Carte géologique au 200.000. Feuilles d'Antioche (avec notice explicative). Damas.

Duchaufour PH., 1952, Espèces forestieres calcicoles et calcifuges. R.F.F., mai 1952, pp. 301-305.

- 1960. Préeis de Pédologie. Masson et Cie, Paris, 438 p.

DURand J. H., 1959. Les sols rouges et les croutes en Algérie. Direction de l'hydraulique et de l'équipement mural. Serv. Etad. scient.

EIG A., 1931. Quelques faits de la phytogéographie palestinienne précédés par des faits sur les notions phytogéographiques. Bull. Soc. Bot. Fr., T. LXXVIII, pp. 297-305.

- 1931. Les eléments et les groupes phytogéographiques auxiliaires dans la flore palestinienne. 1-texte; 2-tableaux analytiques. Dahlem bei Berlin, Verlag des Repertoriums.

- 1933. Ecological and phytosociological observations on Palestine plants. Extr. Beihefte Bot. Centralblatt. Bd. L, Abt. II, pp. 470-496.

EMBERGer L., 1930. La végétation de la Région méditerranéenne. Ersai d'une classification des groupements végétaux. Rev. Gén. Bot., T. 42. 
- 1933. Nouvelle contribution d l'étude de la classification des groupements végétaux. Rev. Gén. Bot. XLV, pp. 1-14.

- 1938. Les arbres du Maroc. Aperçu général sur la végétation du Maroc. Paris.

- 1942. Un projet de classification des elimats au point de vue phytogéographique. Bull. Soc. Hist. Nat. Toulouse LXXVII, pp. 97-124.

- 1952. Sur le Quotient pluviothermique. C. R. Ac. Sc., CCXXXIX, pp. 2508 2510.

- 1954. Projet d'une classification biogéographique des climats. In : Les divisions écologiques du Monde, C.N.R.S., Paris.

- 1958. Afrique du Nord et Australie Méditerranéenne. Climatologie et Mi. croclimatologie. Actes du Colloque de Canberra, UNESCO, pp. 141-147.

- 1959. Bur la notion de climat de transition dans lo domaine du climat méditerranéen. Bull. Serv. Carte Phytog., série B, T. IV, 2, pp. 95-117.

ENGLER A., 1879. Versuch einer Entwicklungsgeschichte der Pflanzenwelt, insbesonder der Florengebiete, seit der Tertiärperiode. Leipzig, Engelmann.

- 1905. Grunderige der Entwiollung der Flora seit der Tertiär-zeit. Result. Sci. Congr. Intern. de Bot., Wien.

Engler A. und Difus L., 1936. Syllabus der Pflanzenfamilien. 11. ergänzte Aufl. Berlin, Borntraeger.

Frori A., 1924. La Flora dell'Isola di Rodi. Extr. l'Agricoltura Coloniale di Firenze, n. 5-6, pp. 161-168.

Fish W. B., 1945. Premières notes sur la météorologie de la Syrie et du Liban. Etud. géol. et géogr. sur le Liban, la Syrie et le Moyen-Orient, T. IV, pp. 91.113.

Flahault Ch., 1937. La distribution géographique des végétaux dans la Région méditerranéenne française. 178 p., Paris.

FournLER Abbé E. M., 1958. La modification des microclimats. In : Climatologie, compte rendu de recherches UNESCO, pp. 140-162.

GAUSSEN H., 1932. Les forêts méditerranéennes et les conditions postglaciaires (analyse pollinique). Bull. Soc. Hist. Nat. Toulouse, T. 64.

- 1954. Géographie des plantes. Collection Armand Colin. Paris, 223 p.

- 1954. Expression des milieux par des formules écologiques. Leur représentation cartographique. In : Les divisions ́́cologiques du monde, C.N.R.S., Paris.

- 1957. Limite et écologie des pays méditerranéens. In : Voyage d'études en France dans la région méditerranéenne. Ed. E.N.E.F., pp. 189-198.

- Les Gymnospermes actuelles et fossiles. fase. XI (Généralités, Genre Pinus), 1960. fasc. IV, ehap. VIII - Les coniférales. Première partie : les Pinoidines. Organes du Sporophytes, 1950-1952. Trav. Lab. Toulouse.

Gìze B., 1956. Carte de reconnaissance des sols du Liban au 1/200.000. Stat. Agr. Libano.Franç., Beyrouth, 52 p.

Gilead H. et Rosenan N., 1958. L'observation elimatologique dans les régions arides. In : Olimatologie, compte rendu de recherches, UNESCO, pp. 199. 207.

Ginnies W. G. Me, 1957. Végétation. In : Guide des travaux de recherches sur la mise en valeur des régions arides. UNESCO, pp. 130-144.

Gombault R., 1945. Aperçu sur la flore de la Syrie, du Liban et de la région d'Antioche (Turquie). Etud. géol. et géogr. sur le Liban, la Syrie et le Moyen-Orient, T. IV, pp. 123-156.

- 1949. Oontribution à la connaissance de la flore du désert syrien. Bull. Soo. Bot. Fr., T. 96, pp. 3-7. 
Goop R., 1947, 1953. The Geography of Flowering Plants, London, Longmans. Green.

GUnNmer Ph., 1952. Le régime forestier au Liban an 2ème siècle. R.F.F., n. 3. Gornochet M., 1955. Logique et dynamique du peuplement végétal. Collection Evolution des seiences, n. 7, Masson et Cie. Paris, 144 p.

Guinochet M. et Casal. P., 1957. Sur l'analyse différentielle de Czekanowski et son application à la Phytosociologie. Bull. Serv. Carte Phytog., série B, carte des groupements végétaux, T. II, 1, pp. 25-33.

Halcer J., 1945. Aperçu sur la Préhistoire de la Syrie et du Liban en 1945. Etudes géol. et géogr. sur le Liban, la Syrie et le Moyen-Orient, T. IV, pp. 49-58.

HenIN S., 1936. Idées actuelles sur l'eau dans le sol et ses rapports avec la plante. Ann. Agr. n. 5, pp. 723-741.

Henin S., Feodoroff A., Gras R., Monnier G., 1960. Le profil oultural (principles de physique du sol). Soc. d'Ed. Ing. Agr., Paris.

KASAPLIGIL B., 1955. The ecological Survey of the vegetation types in Forest and Grazing lands of Jordan. F.A.O.

KAYACIK H., 1954. Pines in Turkey and an investigation about their geographical distribution. (Texte turc, résumé en anglais). Istambul Universitesi, Orman Fakültesi Dergisi, Sayi 1 ve 2.

Kratis A., 1959. Contribution à l'étude systématique, anatomique, phylogénique et écologique des Chénopodiacées de la Syrie. Essai d'anatomie compaŕe. Thèse Fac. Sc. Montepellier, 239 p.

KILlIAN CH., 1931. Recherches écologiques sur les fluctuations saisonnières de la transpiration chez les végétaux du climat méditerranéen.

KoRNAs J., 1959. Succession regressive de la végétation des garrigues sur calcaire compact dans la montagne de la Gardiole près de Montpellier. Comm. n. 145, SIGMA.

Le Hoderou H.-N., 1959. Recherches écologiques et floristiques sur la végétation de la Tunisie méridionale. Thèse Fac. Sc. Montpellier.

LELOUP M., 1957. Forêts. In Guide des travaux de recherche sur la mise en valeur des régions arides, UNESCO, pp. 145-161.

LindBerg H., 1946. Iter Oyprium. Oontributio Ad Cognitionum Florae Insulae Cypri. Acta Societatis scientiarnm Fennicae, Nova Series B, T. II, n. 7, Helsingfors.

Long G., 1954. Contribution a l'étude de la végétation de la Tunisie centrale (thèse). Ann. Serv. Bot. et Agr. Tunis.

- 1957. The Bioclimatology and vegetation of Eastern Jordan. F.A.0. 57/2/1109.

Martonne (Emm. de), 1955. Traité de géographie physique. T. III, Armand Colin, Paris, pp. 1060-1541.

MoLiNIER R., 1954. Les climax cótiers de la France méditerranéenne. Vegetatio, vol. IV, 5, pp. 284.368 .

- 1955. La végétation méditerranéenne dans ses relations avec les conditions de climat et l'action humaine. Bull. Soc. Forest. de Franche Comté et des Provinces de l'Est, 16 p.

Mouterde P., 1946. La végétation arborescente des pays du Levant. Publ. Ecole Franc. d'Ing. Beyrouth.

- 1953. La flore du Djebel Druze. Lechevalier Ed., Paris.

- 1980. Contribution à l'étude de la flore syrienne et libanaise (suite) - genre Astragahs. Bull. Soc. Bot. Fr., T. 107, n. 4-6, pp. 2-13. 
MuIR A., 1951. Notes of the 8oils of Syria. Jour. Soil Sc., vol. 2, n. 2, p. 163. Muzaffrer Selik, 1959. Pinu brutia in der Türkei. Sonderdruck aus Forstw. Cbl. $78 \mathrm{Jg} .1 / 2,1.64$.

NAHAL I., 1959. Expérimentations a entreprendre en Syrie en matière d'Eucalyptus (texte arabe). Minist. Agr., Damas.

- 1959. Oréation et administration des pépinières forestières on Syrie (texte arabe). Minist. Agr. Damas.

- 1960. La végétation forestière naturelle dans le Nord-Ouest de la Syrie. R.F.F., fév. 1960, n. 2, pp. 90-101.

- 1960. Recherches sur la dégradation des forêts de Pinus brutia dans le Baer et le Bassit de Syrie (texte arabe). Serv. Forest. Lattaquié.

- 1961. La garrigue d Quercus calliprinos et Pistacia palaestina du Djebel Alaouite de Syrie. Ann. E.N.E.F., T. XVIII, 3, pp. 409-480.

OPPENHEIMER H. R., 1939. Etudes sur le problème de la reconstitution des chê. naies en Palestine. Pal. Jour. Bot., vol. III, n. l.

OUdin A., 1938. Etude sur le gemmage des pins en France. Ann. E.N.E.F., Nancy.

PaBOT H., 1955, Notes sur un voyage dans la région de Lattaquié. Rapp. 3, Damas.

- 1955. Notes sur un voyage dans les forêts de la région de Lattaquié (Bassit, Jebel Ansarieh). Rapp. 2 (rapp. au Gouvernement syrien).

- 1956. Notes sur un voyage dans les régions de Lattaquié, Kessab, Slenfé, Massiaf, Hama. rap. 11.

- 1957. Rapport au gowvernement de Syrie, sur l'écologie végétale et ses applications. F.A.O., rap. n. 663, Rome.

Papajoannou L., 1935. Les limites de la répartition géographique du $P$. halepensis et $P$. brutia en $N$. D. Chalchidique et leurs associations végétales (texte grec). Extr. Bull. Sc. Nat., Athènes.

- 1936. Eine neue Varietät von Pinus brutia Ten., Pinus brutia Ten. var. Agrophiotii. Extr. des Praktika de l'Académie d'Athènes, 11, p. 14.

- 1954. L'hybridation entre les pins méditerranéens et son influence sur la gemme, surtout en Grèce. $4^{\circ}$ sess. sous-comm. coord. quest. for. Athènes, $10 \mathrm{p}$.

PARDÉ L., 1937. Les conifères. La Maison Rustique, Paris, 294 p.

- 1941. Les feuillus. La Maison Rustique, Paris, 384 p.

- 1957. La productivité des forêts de Pin d'Alep en Franc6. Ann. E.N.E.F., T. XV, 2, pp. 369-413.

Parsa A., 1949-1961. Flore de l'Iran. 8 vol. Téhéran.

Pavillard J., 1935. Eléments de Sociologie végétale (Phytosooiologie). Paris, Hermann et Cie, Ed., 102 p.

Prarrin H., 1954-1958. Sylviculture. - T. I : Bases scientifiques de la Sylviculture, 318 p., 1953. - T. II : traitement des forêts, 423 p., 1954. - T. III : travaux forestiers, 405 p., 1958. - Ed. E.N.E.F., Nancy.

Philippis A. DE. Les bases écologiques de la sylviculture méditerranéenne. (F.A.0./48.12/452 f. Trav. Med. F.A.0./E.FC/HSC/16).

Post G. E. and Dinsmore J. E., 1932-33. Flora of Byria, Palestine and Binai. Ed. 2, American Press, Beirut.

PodRTET J., 1951. Les sapins méditerranéens. In : Les repeuplements artificiels, pp. 97-100. E.N.E.F., Naney.

Remenberg A., 1947. The soils of Palestine. Translated by C. L. Whittles H., Thomas Murby et Co. London.

- 1952. The soils of Syria and the Lebanon. Jour. of Soil Sc. B(1): 68-88. 
Reynadd-Beatverte M. A., 1936. Le milieu et la vie en commun des plantes. Paul Lechevalier Ed., Paris, 233 p.

Salvador, 1931. Les forêts dans les pays méditerranéens. Bull. le Chêne.

SELod Y. I., 1961. Bioclimats et végétation du Pakistan occidental. Thèse, Fac. Sc. Univ. Toulouse.

Stoddart L. A. and SMath A. D., 1955. Range Management. MeGraw-Hill, Book Company Inc., New York, 433 p.

Täckholm V. and DRAR M., 1941-50-54. Flora of Egypt. vol. I, II, III. Bull. Fac. Sc. Cairo Univ., No. 17, 28, 30. Cairo.

ThíkBadt J., 1936. F'lore Libano-Syrienne. $1^{\circ}, 2^{\circ}$ partie. Le Caire. Impr. Inst. Franç. d'Archéol. Orient.

- 1953. Flore Libano-Syrienne. $3^{3}$ partie. Ed. C.N.R.S. Paris.

ThORNTHWAITE C. W., 1958. Introduction to arid zome climatology. In : Climatologie et microclimatologie, Actes Coll. Canberra; UNESCO, pp. 15-22.

VAUMAS E., 1954. Le Liban (montagne libanaise, Belcaa, Anti-Liban, Hermon, Haute Galilée libanaise). Etude de géographie physique. Paris, Firmin-Didot.

- 1954. Montagnes du Proche-Orient, l'Amanus et le Djebel Ansarieh. Etude morphométrique. Rev. de géogr. alpine, XLII, I.

- 1956. Sur la strueture et sur la surface d'érosion polycyclique du Djebel Ansarieh (Syrie). Ibid. T. 242, p. 1632.

- 1956. Le Djebel Ansarieh. Etude morphologique. Bull. Soc. de Géogr. d'Egypte, T. XXIX.

- 1958. Le massif du Djebel Akraa (Cassius), étude morphologique. Bull. Soc. Géogr. d'Egypte, T. XXXI.

- 1960. Le Djebel Ansarieh. Etudes de géographie humaine. Rev. gégr. alpine, T. XI.VIII.

Verner A., 1958. Climates and vegetation. In : Climatologie. Rev. of Research, UNESCO, pp. 75-101.

Weaver J. and Crements F. E., 1938. Plant Ecology. MeGraw Hill, Book Comp. Ine., New York and London, $601 \mathrm{p}$.

Wetzer R. et HaLler J., 1945-1948. Le Quaternaire eốtier de la région de Tripoli (Liban). In : Etudes géologiques et géographiques sur le Liban, la Syrie et le Moyen-Orient, T. IV, pp. 1-48, Beyrouth.

Woodbury A. M., 1953. Principles of General Ecology. New York, Blakiston Comp. Inc., 503 p.

ZoHary M., 1951. The Arboreal Flora of Israel and Transjordan and its ecological and phytosociological significance. Imper. Forest. Inst. Univ. Oxford, n. 26.

- 1952. A monographical study of the genus Pistacia. Pal. J. Bot., 5: 187-228.

ZOHARY M. and ORSHANSKY G., 1947. The vegetation of the Huleh Plain. Pal. Jour. Bot., vol. IV, 90-104.

\section{RESUME}

Cette étude concerne les montagnes du Baer-Bassit et des Alaouites qui sont situées dans le N.W. de la Syrie, sur le bord occidental de la Péninsule Arabique. Le Djebel Alaouite est formé essentiellement de calcaires jurassiques et crétacés. Il est orienté Nord-Sud et culmine aux environs de $1580 \mathrm{~m}$. Le Baer-Bassit est un pays montagneux et formé essentiellement de roches vertes (peridotites pyroxéniques, gabbros, dolórites). 
Nous y avons reconnu les formes et variantes suivantes du climat méditerranéen : elimat subhumide chaud, elimat humide tempéré et froid, climat perhumide froid.

Nous avons montré la nécessité de la connaissance des roches-mères pour l'étude de la végétation et de son évolution dans ces régions. Nous avons également étudié la genèse ot la classification des sols issus des différentes roches-mères, ainsi que leur dégradation sous l'action de l'homme et l'influence de leur composition physico-chimique sur la répartition et le développement de la végétation.

Nous avons conclu que la flore du Baer-Bassit et du Djebel Alaouite présentait déjà au Pliocène ses traits les plus importants et que les modifications pluviales survenues au Quaternaire ont eu une grande répercussion sur la distribution de la flore et laissé des empreintes très visibles de nos jours.

Nous avons étudie trois espèces forestières principales : Pinus brutia Ten., Dedrus libani A. Rich. et Abies cilicica (Ant. et Ky.) De Tchih. des points de vue botanique, autoécologique et forestier et dégagé les règles sylvicoles à suivre pour l'aménagement et l'exploitation rationnelle des forêts de Pinus brutia encore en bon état et la reconstitution des sapinières et des cédraies en voie de disparition.

Nous avons terminé par une étude synsystématique et synécologjque de l'association arborescente la plus importante de Syrie, le Pistacieto-Quercetum calliprini et avons montré l'intérêt pratique que peut présenter pour le fore. stier la connaissance des phases de successions phytodynamiques progressives et régressives.

\section{SUMMARY}

This study deals with the Baer-Bassit and Alaouite mountains situated in the N-W of Syria on the Western Coast of the Arabian Peninsula. The Djebel Alaouite is essentially formed of jurassic and cretaceous calcaireous rocks. It is oriented N-S and culminates on $1580 \mathrm{~m}$. The Baer-Bassit is a hilly country and is formed essentially of green-rocks (Péridotites pyroxéniques, gabbros, dolérites).

We have distinguished in these regions the following forms and variants of the mediterranean climate : hot sub-humid climate, cold and temperate humid climate and cold perhumid climate.

We have shown the necessity of the knowledge of mother-rocks for the study of vegetation and its evolution in these regions. We have equally studied the genesis and the classification of the soils evolved from the different motherrocks as well as their degradation by the action of man and the influence of their physico-chemical composition on the repartition and development of vegetation.

We concluded that the flora of the Baer-Bassit and Djebel Alaouite already showed its most caracteristic traits at the Pliocene and that the pluvial mo. difications, that influenced it during the quaternary, have had a great influence on the distribution of the flora and have left very visible marks upto the present day.

We have studied three principal forest species : Pinus brutia Ten., Cedrus Vibani A. Rich. and Abies cilicica (Ant. et Ky.) De Tehih. from the botanical, autoecological and forest point of vue and we have brought to light the silvi- 
cultural rules that should be followed for the rational management and exploitation of the forests of Pinus brutia, still in good condition, and the reconstitution of the forests of Abies cilicica and Cedrus libani on way to extinction.

We have concluded with a synsystematic and synecologic study of the most important tree association of Syria, the Pistacieto-Quercetum calliprini and have pointed out the practical interest that holds for the forester the knowledge of the progressive and regressive phases of phytodynamic successions.

\section{SAMENVATTING}

Deze studie heeft betrekking op de bergen van Baer-Bassit en op het Alaouite-gebergte, gelegen in het noordwesten van Syrië aan de westelijke rand van het Arabisch Schiereiland. Het Alaouitegebergte is voornamelijk samengesteld uit kalkhoudend gesteente, gevormd tijdens Jura en Krijt; het loopt van noord naar zuid en bereikt een maximum-hoogte van $1580 \mathrm{~m}$. BaerBassit is een heuvelachtig gebied, voornamelijk bestaande uit pyroxeenhoudend peridotiet, gabbros en doleriet.

De volgende varianten van het mediterrane klimaat worden onderschejden : heet, min of meer vochtig klimaat; koud, matig vochtig klimaat en koud, zeer vochtig klimaat.

Aangetoond wordt de noodzakelijkheid van de kennis van de moedergesteenten als basis voor de bestudering van de vegetatie en haar ontwikkeling in deze gebjeden. Bestudeerd werden het ontstaan en de indeling van de bodemtypen, die zich nit de verschillende moedergesteenten ontwikkeld hebben, alsmede hun afbraak onder invloed van de mens en de invloed van hun physisch-ehemische samenstelling op de verspreiding en de ontwikkeling van de vegetatie.

Geconcludeerd wordt dat de flora van Baer-Bassit en die van het Alaouitegebergte haar belangrijkste kenmerken reeds vertoonde in het Plioceen, en dat de regenperioden tijdens het Quartair een grote invloed hebben gehad op de verspreiding van de flora en tot heden daarop duidelijk een stempel gedrukt hebben.

Bestudeerd werden voorts de drie voor de bosbouw belangrijkste boomsoorten : Pinus brutia Ten., Cedrus libani A. Rich. en Abies cilicica, zowel nit botanisch, uit autoecologisch als uit bosbouwkundig oogpunt. Tevens worden richtlijnen gegeven om te komen tot een zo rationeel mogelijk beheer en een zo gunstig mogelijke exploitatie van de Pinus brutia-bossen, welke nog in goede staat verkeren, alsmede voor het herstel van de Abies cilicica- en Cedrus libani-bossen, welke dreigen te verdwijnen.

Besloten wordt met de bestudering van de synsystematiek en de synoecologie van de belangrijkste bosassociatie van Syrië, het Pistacieto - Quercetum calliprini. Voorts wordt aangetoond het practische belang, dat voor de bosbouwer gelegen is in de kennis van de phytodynamische successies, zowel progressief als regressief.

\section{RIASSUNTO}

Il presente studio riguarda le montagne del Baer-Bassit e delle Alaouites, situate nella parte nord-occidentale della Siria, al limite ovest della penisola araba. Il Gebel Alaouite è formato principalmente da calcari giurassici e cretacei ed è orientato in direzione nord-sud raggiungendo circa $\mathrm{m}$ 1580. Il BaerBassit è una regione montuosa formata essenzialmente di rocce verdi (perido, titi pirosseniche, gabbri, doleriti). 
L'autore ha riconoseiuto le seguenti forme e varianti del elima mediterraneo : clima subumido caldo, clima umido temperato e freddo, clima perumido freddo.

L'autore mostra la necessita della conoscenza delle rocce madri per lo studio della vegetazione e della sua evoluzione in queste regioni. Egli studia quindi la genesi e la classificazione dei terreni prodotti dalle differenti rocce madri, nonché la loro degradazione per opera dell'uomo e l'influenza della loro composizione chimico-fisica sulla distribuzione e aullo sviluppo della vegetazione.

L'autore sostiene che la flora del Baer-Bassit e del Gebel Alaouite presentava già nel Pliocene le caratteristiche più importanti e che le modificazioni pluviali sopraggiunte nel Quaternario hanno avuto una grande ripercussione sulla distribuzione della flora ed hanno lasciato impronte chiaramente visibili anche oggi.

L'autore esamina quindi le tre principali specie forestali : Pinus brutia Ten., Cedrus libani A. Rich. e Abies cilioica (Ant. et Ky.) De Tchih. sia dal punto di vista botanico che da quello antoecologico e forestale, e delinea le norme selvicolturali per il trattamento e l'utilizzazione razionale delle foreste di Pinus brutia ancora in buono stato, e per la ricostituzione delle foreste di Abies cilicica e di Cedrus libani in via di estinzione.

L'autore termina quindi con uno studio sinsistematico e sinecologico della più importante associazione arborescente della Siria, il Pistacieto-Quercetum calliprini, e mostra quale interesse pratico presenti per il forestale la conoscenza delle fasi di successione dinamica progressive e regressive.

\section{TABLE DES MATIE RES}

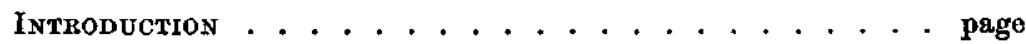

Cr. 1 : Situation géographique et orographique du Baer-Bassit et du Djebel Alaouite . . . . . . . . . . . . . . . "

1) Situation géographique . . . . . . . . . . . . "

2) Paléogéographie . . . . . . . . . . . . . .

3) Orographie . . . . . . . . . . . . . . . . . . . . *

3.1. Djebel Alaouite. . . . . . . . . . . . . . . . "

Cr. 2 : Caractéristiques du milieu dans le Baer-Bassit et le Djebel Alaouite

I - LF CLIMAT

1) Classification biogéographique des elimata - "le bioclimat ". .

2) Bioclimats du Baer-Bassit et du Djebel Alaouite . . . . .

3) Comparaison du régime climatique du Baer-Bassit et du Djebel Alaouite avec celui de la Méditerranée occidentale :

4) Les étages bioclimatiques dans le Baer-Bassit et le Djebel Alaouite 
II - LES ROCHES-MHRES . . . . . . . . . . . . . . . page

1) Importance de la connaissance des roches-mères pour l'étude de la végétation dans le Baer-Bassit et le Djebel Alaouite .

2) Les principaux types de roches-mères . . . . . . . . . 2.1. Les roches vertes . . . . . . . . . . . . . . . " 2.1.1. Groupe des péridotites pyroxéniques . . . . . 2.1.2. Groupe des gabbros, dolérites et diorites . . .

2.2. Les roches-mères calcaires . . . . . . . . . . . . . 2.2.1. Le calcaire compact . . . . . . . . . . 2.2.2. Le calcaire dolomitique . . . . . . . . . . . 2.2.3. La marne crayeuse . . . . . . . . . . . . . "

2.3. Les roches-mères basaltiques . . . . . . . . . . . . *

2.4. Les sols fossiles jouant le rôle de roches-mères . . . . 2.4.1. La Terra rossa . . . . . . . . . . . . . .

III - LES SOLS

1) Les sols formés sur péridotites pyroxéniques et serpentines

1.1. Propriétés et types

508

508

1.2. Végétation ... . . . . . . . . . . . " 512

1.3. Dégradation . . . . . . . . . . . . . . . . . 512

2) Les sols formés sur gabbros . . . . . . . . . . . . . . 513

2.1. Propriétés et types . . . . . . . . . . . . \& 513

2.2. Végétation . . . . . . . . . . . . . . ., 514

2.3. Dégradation . . . . . . . . . . . . . . * 514

3) Les sols formés sur calcaire compact et Terra rossa . . . . " 515

3.1. Propriétés et types . . . . . . . . . . . . . . . 515

3.1.1. Sols bruns méditerranéens sur Terra rossa . . \$ 515

3.1.2. Rendzines rouges . . . . . . . . . . . " 516

3.1.3. Sols humiques carbonatés . . . . . . . . . . " 516

3.2. Végétation . . . . . . . . . . . . . . . . . " 516

3.3. Dégradation des sols bruns méditerranéens sur Terra rossa " 517

4) Les sols formés sur marne crayeuse . . . . . . . . . . . 518

4.1. Propriétés et types . . . . . . . . . . . . . . . . . 518

4.2. Végétation . . . . . . . . . . . . . . . " 510

4.3. Dégradation des sols bruns calcaires et des rendzines . . $\quad 519$

5) Les sols formes sur calcaire dolomitique . . . . . . . . $\$ 520$

6) La systématique des sols forestiers du Baer-Bassit et du Djebel

Alaouite ................

IV - INFLUENCE DES VERSANTS . . . . . . . . . . 523

V - L'HOMME EN TANT QUE FACTEUR DU MULIEU . . . . " 525

1) Groupements elimaciques - discussion ......... * 525

2) Le rôle de l'homme dans l'évolution des groupements végétaux dans le Baer-Bassit et le Djebel Alaonite . . . . . . $\quad 526$

Ch. 3 : Caractéristiques floristiques et phytogéographiques du Baer-Bassit et du Djebel Alaouite . . . . . . . . . * 528

I - Notions gÉNERALES FlORISTICO-PHYTOGÉGRAPHIQdES . . . 529 
1) Royaumes floraux . . . . . . . . . . . . . . . . . . . page 529

2) Régions phytogéographiques et Provinces . . . . . . . . 530

3) Elément . . . . . . . . . . . . . . . . . . " 532

4) Enclaves . . . . . . . . . . . . . . . . . . . . . 534

5) Irradiations . . . . . . . . . . . . . . . . . . . . . . 536

6) Plantes de liaison . . . . . . . . . . . . . . . . . . . " 535

7) Plantes plurirégionales ou cosmopolites ou ubiquistes . . . " 536

II - Les f́léments méditerRanéns DaNS LE BaER-Basgit ET LE DJebel AlaOUtte .............. 537

1) Sous-élément omniméditerranéen . . . . . . . . . . , 537

a) Espèces omniméditerranéennes . . . . . . . . . 537

b) Espèces subméditerranéennes . . . . . . . . . . . . . . 539

2) Sous-élément méditerranéo-oriental .......... . 540

a) Espèces méditerranéo-orientales . . . . . . . . . . , 540

b) Espéces aubméditerranéo-orientales . . . . . . . . . " 542

3) Sous-élément méditerranéo-occidental . . . . . . . . . \$ 544

4) Sous-élément méditerranéo-septentrional . . . . . . . . " 544

a) Espèces méditerranéo-septentrionales . . . . . . . . . . . 544

b) Espèces subméditerranéo-septentrionales . . . . . . . . 54

5) Sous-élément méditerranéo-méridional . . . . . . . . " 545

III - LES ENCLAVES ................." 545

a) Les enclaves nordiques . . . . . . . . . . . . . . " 545

b) Les enclaves saharo-sindiennes . . . . . . . . . " 546

IV - Les plantes de liaison . . . . . . . . . . . * 547

1) Plantes de liaison méditerranéo-irano-touraniennes . . . . " 547

a) Espéces méditerranéo-irano-touraniennes ........ 547

b) Espèces sub-méditerranéo-irano-touraniennes ...... . 548

c) Espèces méditerranéo-orientales-irano-touranionnes . . . 549

2) Plantes de liaison eurosibériennes-boréoaméricaines-méditerranéo-irano-touraniennes ............ 550

V - Les planteg pluRirégionales ou cosmopolites . . . 551

a) Espèces boréo-tropicales . . . . . . . . . . . . 551

b) Espèces subtropico-tropicales . . . . . . . . . . . . 552

VI - LeS Plantes ENdÉmiques . . . . . . . . . . 553

VII - Essai sur le développement de la flore du BaERBastit eT dU DJebel AlaOdTE . . . . . . . . , 565

1) Paléoclimatologie et préhistoire ........... .

2) Développement de la flore du Baer-Bassit et du Djebel Alaouite $\quad$ \#58

2.1. La flore au Tertiaire . . . . . . . . . . . , 558

2.2. La flore au Quaternaire . . . . . . . . . . . " 558

VIII - Caracteres DE LA FLORE DU BAER-Bassit ET DU DJebel Ala 
CH. 4 : Etude des trois espèces forestières prineipales (répartition gégraphique, étude botanique, autoécologique et forestière) page

I - Pinds hrutia Ten. ................. . . . 566

1) Aire géographique du Pinus brutia Ten. . . . . . . 566

2) Position systématique du Pinus brutia Ten. . . . . . . * 569

3) Considérations phylétiques .............. 571

4) Caractères botaniques et dendrologiques . . . . . . . . . 572

5) Les peuplements élites de Pinus brutia dans le Baer-Bassit " 574

6) Autoécologie du Pinus brutia dans le Nord-Ouest de la Syrie " 576

6.1. Altitude et exposition . . . . . . . . . . . . 576

6.2. Conditions climatiques . . . . . . . . . . . . . 576

6.3. Conditions édaphiques . . . . . . . . . . . . . . " 576

7) Tempérament du Pinus brutia . . . . . . . . . 577

8) Types de peuplements de Pinus brutia dans le Nord-Ouest de

la Syrie ...................." 577

8.1. La futaie pure régulière . . . . . . . . . . . . " 578

8.2. La futaie pure jardinée . . . . . . . . . . . . . . " 578

8.3. Les peuplements mélangés . . . . . . . . . . . * 579

8.3.1. Peuplements de Pinus brutia et Quercus pseudocerris . . . . . . . . . . . . . * 679

8.3.2. Peuplements de Pinus brutia et Quercus calliprinos . . . . . . . . . . . .

9) Importance du sous-étage de feuillus dans les forêts de Pinus

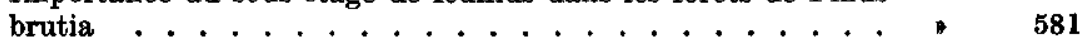

II - Cedrus ribant A. RICH. . . . . . . . . . . . . " 582

1) Aire géographique du genre Cedrus (Trew.) Link . . . . . " 582

2) Systématique du genre Cedrus (Trew.) Link . . . . . . . \$ 584

3) Caractères botaniques et dendrologiques . . . . . . . . 585

4) Autoécologie du Cedrus libani dans le Djebel Alaouite . . . " 587

4.1. Altitude et exposition ............... , 587

4.2. Conditions climatiques . . . . . . . . . . . . 588

4.3. Conditions édaphiques ... . . . . . . . . . . . " 588

4.4. Tempérament. . . . . . . . . . . . . . . . . . 589

5) Les peuplements de Cèdres dans le Djebel Alaouite . . . . " 590

6) Les forêts de Cèdres de Syrie et du Liban dans l'histoire . . $\quad 591$

III - ABIEs cilicica (ANT. ET Kт.) DE TchihatchefF . . . $\quad 592$

1) Aire géographique de l'Abies cilicica . . . . . . . . " 592

2) Caractères botaniques et dendrologiques . . . . . . . . " 594

3) Autoécologie de l'Ábies cilicica dans le Djebel Alaouite. . $\quad 595$

3.I. Altitude et exposition ............... " 595

3.2. Oonditions climatiques . . . . . . . . . . . . . . 595

3.3. Conditions édaphiques . . . . . . . . . . . . . . . . 596

3.4. Tempérament . . . . . . . 596

4) Les peuplements d'Abies cilioica dans le Djebel Alaonite. . . 597 
Cн. 5 : La garrigue à Quercus calliprinos Webb et Pistacia palaestina Boiss. du Djebel Alaouite (Pistacieto-Quercetum calliprini Alaouitaea Nahal) . . . . . . . . page

1) Introduction . . . . . . . . . . . . . . . . . " 598

2) Définition du terme garrigue . . . . . . . . . . . . 599

3) Quercus calliprinos Webb et Pistacia palaestina Boiss. . . 600

3.1. Quercus calliprinos Webb . . . . . . . . . . . औ 600

3.1.1. Caractères botaniques et dendrologiques . . . . " 601

3.1.2. Autoécologie et tempérament . . . . . . . . " 607

3.2. Pistacia palaestina Boiss. . . . . . . . . . . . . . " 608

3.2.1. Caractères botaniques et dendrologiques . . . . " 608

3.2.2. Autoécologie et tempérament . . . . . . . . 609

4) Position du Pistacieto-Quercetum calliprini dans la végétation zonale de la Méditerranée orientale . . . . . . . . . . .

4.1. Etages de végétation dans la Montagne des Alaouites..

4.1.1. Versant Ouest . . . . . . . . . . . .

4.1.2. Versant Est . . . . . . . . . . . . . "

4.2. Etages de végétation dans le Liban . . . . . . . . .

4.2.1. Versant Ouest . . . . . . . . . . . . "

4.2.2. Versant Est . . . . . . . . . . . . . . . "

5) Position du Pistacieto-Quercetum calliprini du Djebel Alaouite dans le Pistacieto-Quercetum calliprini orientale . . . . .

6) La composition floristique du Pistacieto-Quercetum calliprini alqouitaea - Les relevés . . . . . . . . . . . . .

6.1. Localisation des relevés . . . . . . . . . . . . . . .

6.2. Méthode des relevés . . . . . . . . . . . . . . . .

6.3. Choix des relevés . . . . . . . . . . . . . . . . .

6.4. Echantillonnage sur les terrain . . . . . . . . . . . "

7) Position systématique du Pistacieto-Quercetum calliprini . . " 615

8) Subdivisions du Pistacieto-Quercetum calliprini on Syrie . . 616

9) Caractéristiques synécologiques du Pistacieto-Quercetum calliprini alaouitaea

9.1. Etages bioclimatiques . . . . . . . . . . . . . " 617

9.2. Sols . . . . . . . . . . . . . . . . " 617

10) La signification écologique de la composition floristique de la garrigue à Quercus calliprinos et Pistacia palaestina . .

11) Facteurs de variation de la composition floristique de la garrigue ................

11.1. Altitude . . . . . . . . . . . . . .

11.2. Exposition . . . . . . . . . . . . . . . . "

11.3. Origine de la garrigue . . . . . . . . . . . . . . . .

11.4. Densité de la garrigue . . . . . . . . . . . . . . . "

12) Phénologie . . . . . . . . . . . . . . . . . . "

13) Adaptation des especes de la garrigue au substratum rocheux calcaire

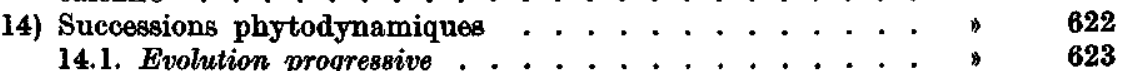
14.2. Evolution regressive .............. ". 626 
15) Importance de la connaissance des phases de successions phytodynamiques pour le forestier . . . . . . . . . page 626

Blbliographie . . . . . . . . . . . . . . . . " 628

RESUMÉ . . . . . . . . . . . . . . . " 633

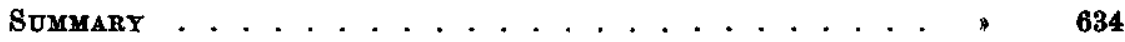

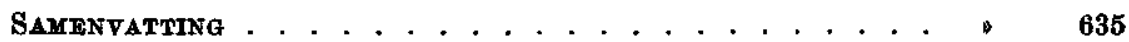

Riassunto ..................... . . 635 